Aus dem Institut für Ethik und Geschichte der Medizin (Prof. Dr. phil. A. Simon)

der Medizinischen Fakultät der Universität Göttingen

\title{
Palliative Sedierung bei amyotropher Lateralsklerose
}

\author{
Ergebnisse einer Befragung von ÄrztInnen aus den \\ Bereichen Neurologie und Palliativmedizin
}

\author{
INAUGURAL-DISSERTATION \\ zur Erlangung des Doktorgrades \\ der Medizinischen Fakultät der \\ Georg-August-Universität zu Göttingen
}

vorgelegt von

Laura Salzmann

aus

Bad Hersfeld

Göttingen 2020 
Dekan:

\section{Betreuungsausschuss}

Betreuer:

Ko-Betreuer:

\section{Prüfungskommission}

Referent:

Prof. Dr. A. Simon

Korreferent:

Promotor-Vertreterin:
Prof. Dr. W. Brück

Prof. Dr. A. Simon

Prof. Dr. B. Alt-Epping
Prof. Dr. M. Schön

Datum der mündlichen Prüfung: 25.08.2021 
Hiermit erkläre ich, die Dissertation mit dem Titel "Palliative Sedierung bei amyotropher Lateralsklerose. Ergebnisse einer Befragung von ÄrztInnen aus den Bereichen Neurologie und Palliativmedizin" eigenständig angefertigt und keine anderen als die von mir angegebenen Quellen und Hilfsmittel verwendet zu haben.

Göttingen, den 


\section{Inhaltsverzeichnis}

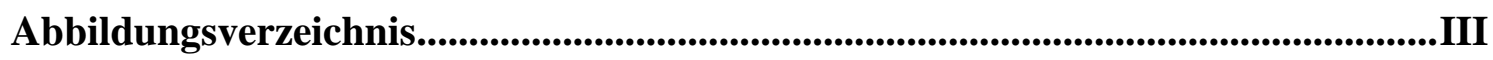

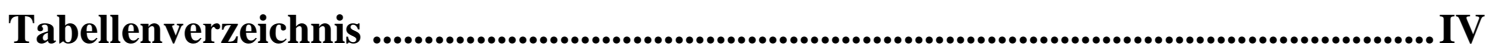

Abkürzungsverzeichnis .................................................................................................................... V

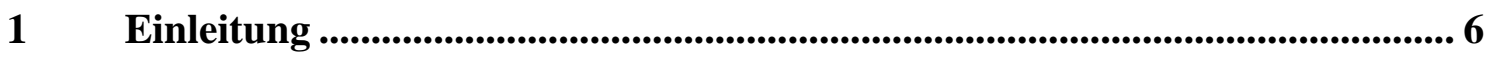

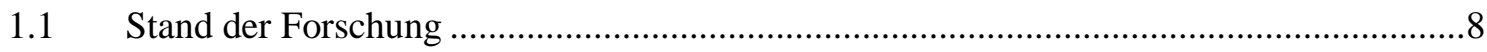

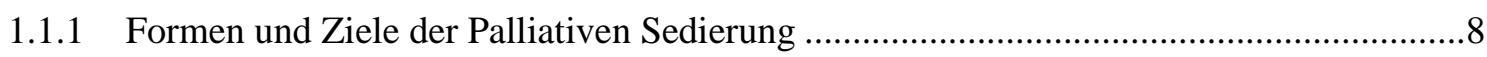

1.1.2 Normative Voraussetzungen der Palliativen Sedierung...................................................

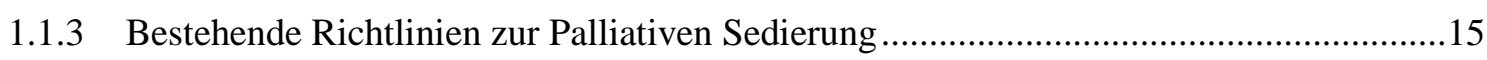

1.1.4 Medizinethische Kontroversen bezüglich der Palliativen Sedierung ..............................19

1.1.5 Palliative Sedierung bei PatientInnen mit amyotropher Lateralsklerose .........................24

1.1.6 Zusammenfassung des aktuellen Forschungsstandes ....................................................29

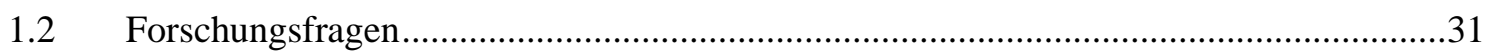

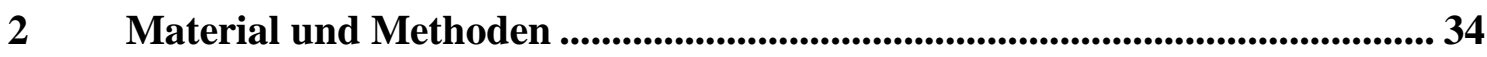

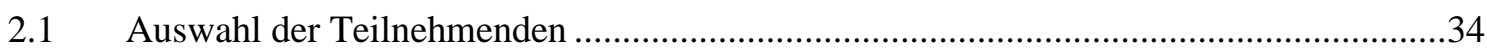

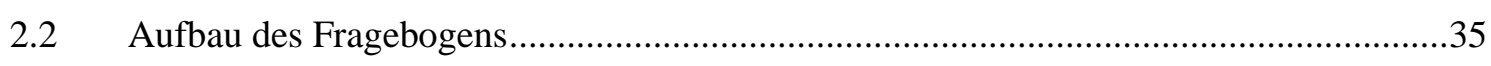

2.3 Erstellung des Fragebogens und Durchführung der Befragung ........................................38

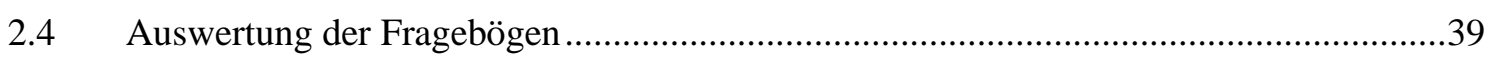

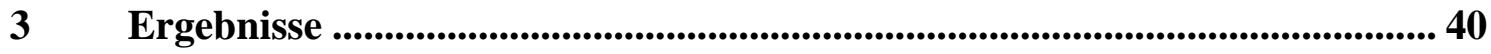

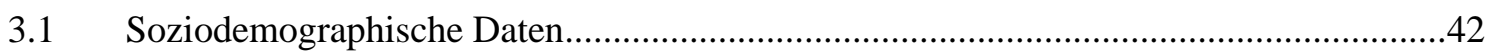

3.2 Allgemeine Fragen zur Palliativen Sedierung und zur Behandlung von ALS-

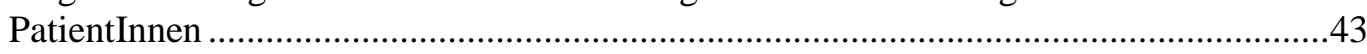

3.3 Fallbeispiel 1.1: Tiefe kontinuierliche Sedierung bei körperlicher Symptomatik ............48

3.4 Fallbeispiel 1.2: Tiefe kontinuierliche Sedierung bei körperlicher Symptomatik mit Verzicht auf künstliche Ernährung ............................................................................53

3.5 Fallbeispiel 2.1: Tiefe kontinuierliche Sedierung bei psychischer Symptomatik ............61

3.6 Fallbeispiel 2.2: Tiefe kontinuierliche Sedierung bei psychischer Symptomatik mit Verzicht auf künstliche Ernährung ..............................................................................66

3.7 Fallbeispiel 3: Elektive Beatmungsbeendigung unter Palliativer Sedierung ....................74

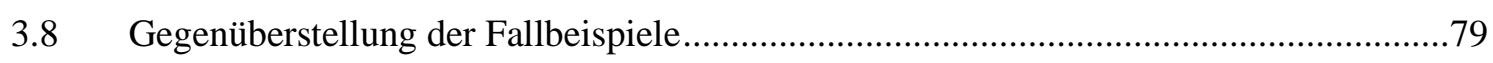

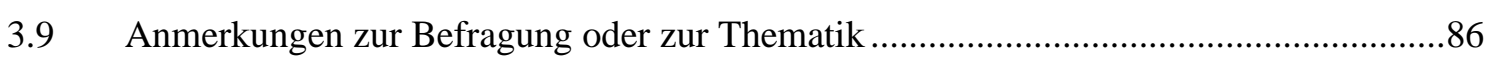

3.9.1 Kritik an der Formulierung der Fragen, Fallvignetten oder der Items.............................86

3.9.2 Hinweis auf die Schwierigkeit, ein ethisch und medizinisch komplexes Thema im

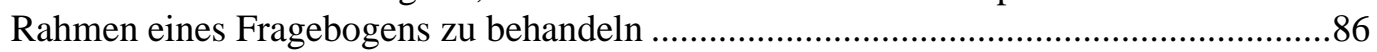

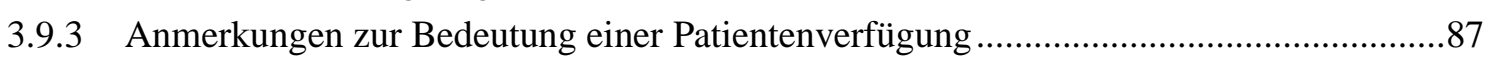

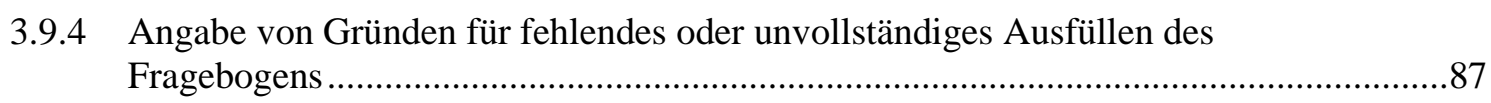




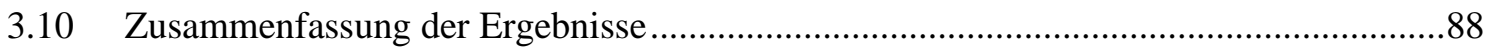

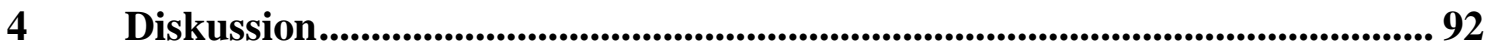

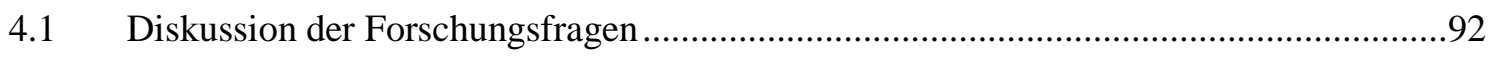

4.1.1 Kommt die Palliative Sedierung bei der Behandlung von ALS-PatientInnen zum Einsatz?

4.1.2 Inwiefern würden behandelnde ÄrztInnen dem Wunsch von ALS-PatientInnen nach kontinuierlicher und tiefer Sedierung zustimmen, um einerseits körperliche und andererseits psychische Symptome zu lindern?

4.1.3 Inwiefern beeinflusst der gleichzeitige Wunsch nach Verzicht auf künstliche Ernährung die Bewertung der Palliativen Sedierung?

4.1.4 Wie beurteilen behandelnde ÄrztInnen den PatientInnenwunsch nach elektiver Beatmungsbeendigung bei gleichzeitiger Palliativer Sedierung?

4.1.5 Inwiefern wird das Antwortverhalten durch die Berufserfahrung und die Aus- und Weiterbildung der teilnehmenden ÄrztInnen beeinflusst?.

4.2 Stärken und Schwächen der Untersuchung.

$5 \quad$ Fazit

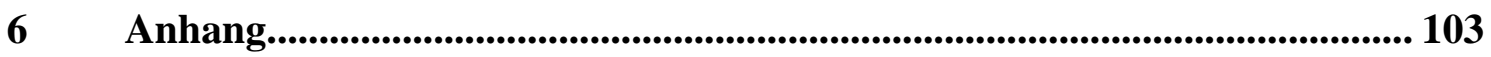

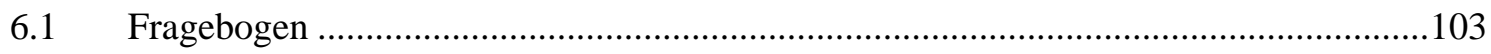

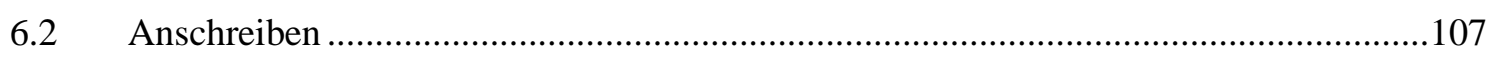

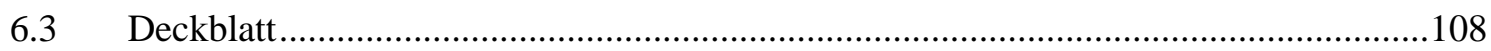

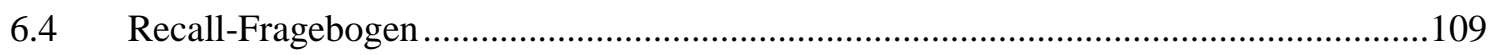

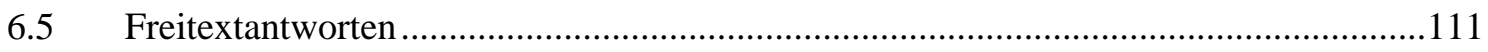

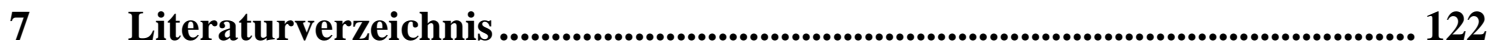




\section{Abbildungsverzeichnis}

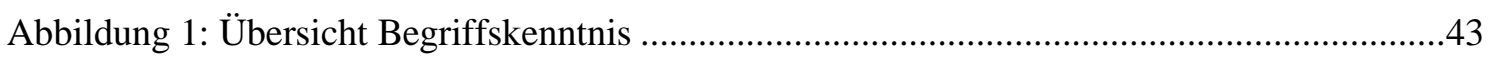

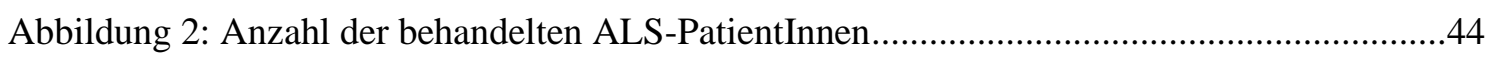

Abbildung 3: Übersicht Erfahrung mit ALS-Behandlung ...........................................................45

Abbildung 4: Übersicht Erfahrung mit Palliativer Sedierung bei ALS........................................46

Abbildung 5: Freitextantwort zur Indikation für Palliative Sedierung bei ALS .........................47

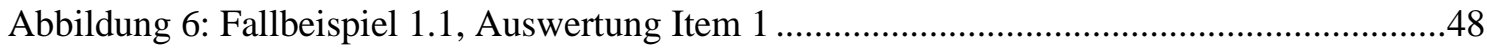

Abbildung 7: Fallbeispiel 1.1, Auswertung Item 2 ...............................................................

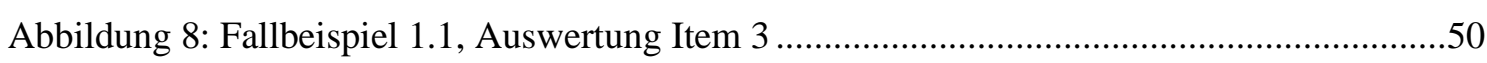

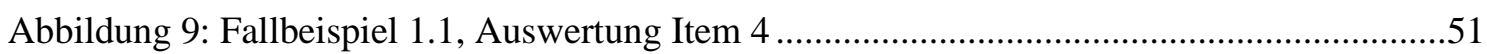

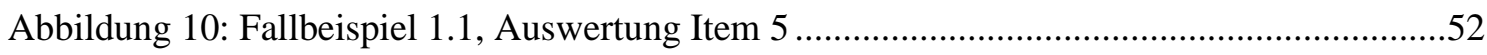

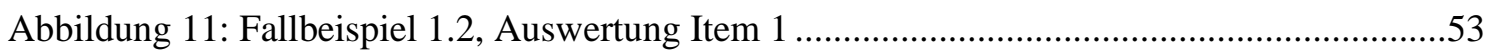

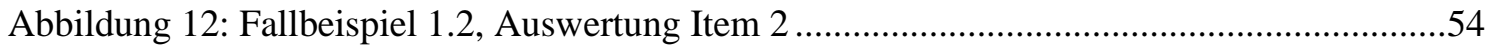

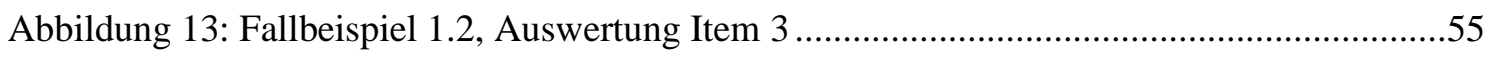

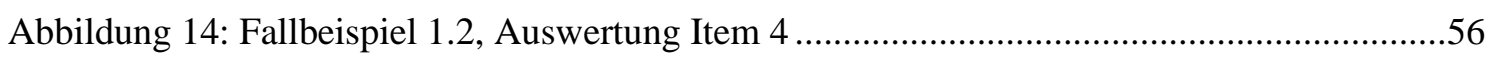

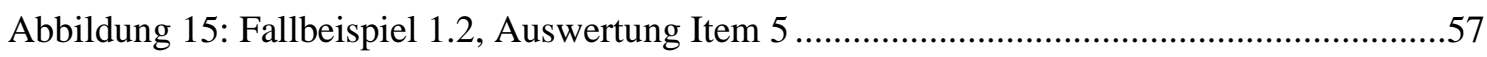

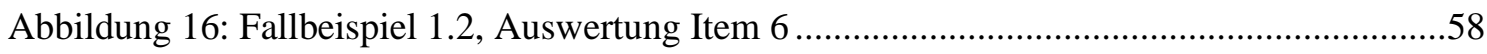

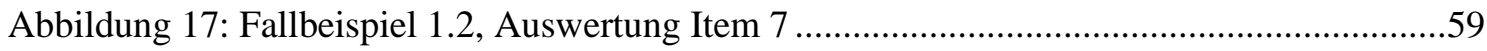

Abbildung 18: Fallbeispiel 1.2, Auswertung Zusatzfrage ..........................................................60

Abbildung 19: Fallbeispiel 2.1, Auswertung Item 1 ...............................................................61

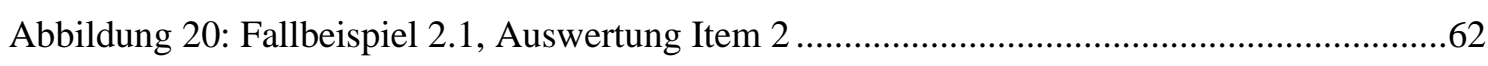

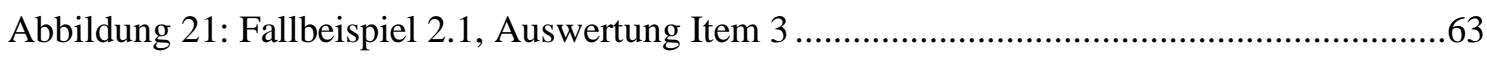

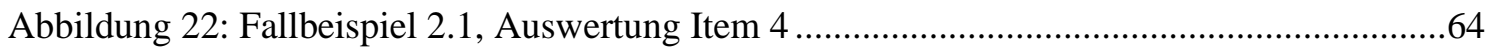

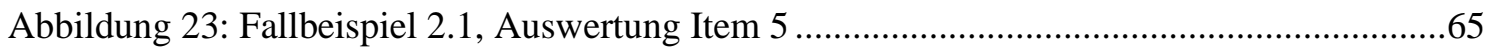

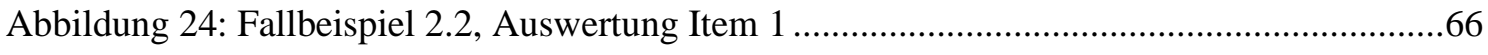

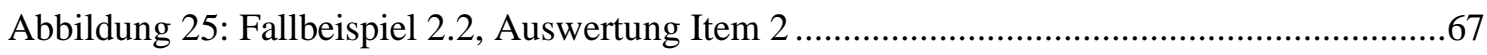

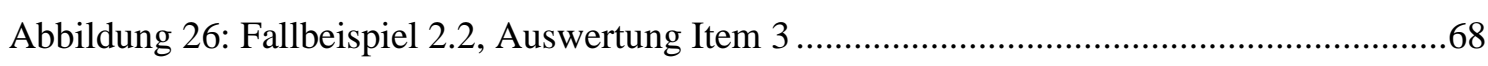

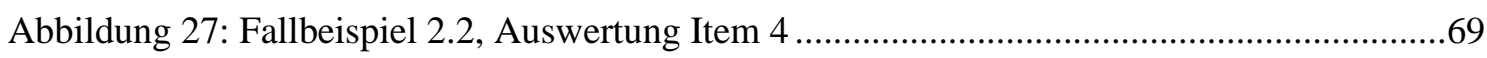

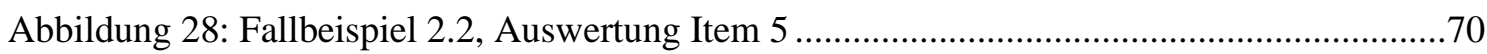

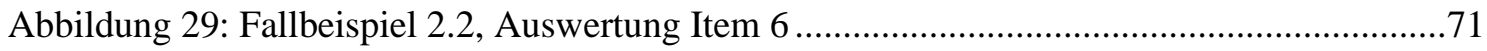

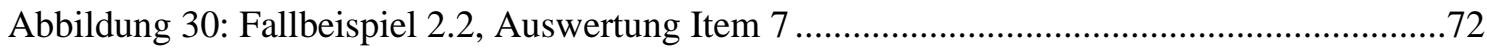

Abbildung 31: Fallbeispiel 2.2, Auswertung Zusatzfrage..........................................................73

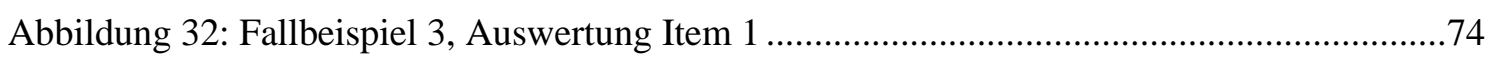

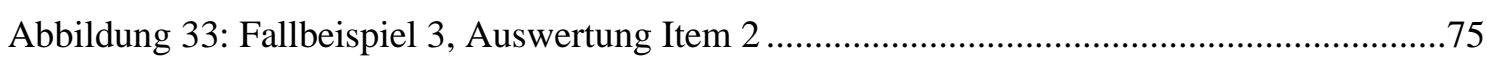

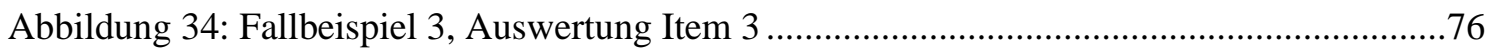

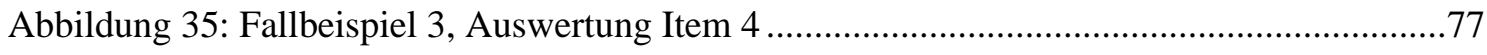

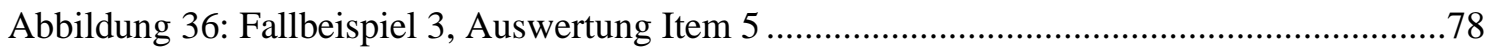

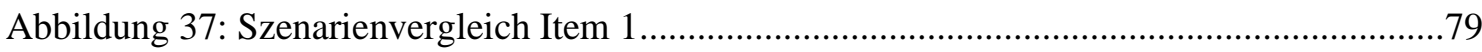


Abbildung 38: Szenarienvergleich mit Differenzierung nach "Zusatz-Weiterbildung Palliativmedizin" Item 1

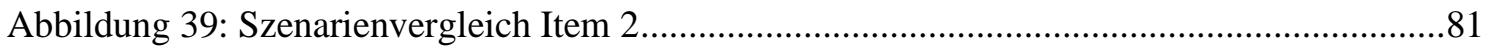

Abbildung 40: Szenarienvergleich mit Differenzierung nach "Zusatz-Weiterbildung

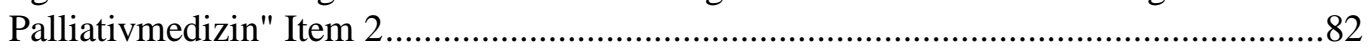

Abbildung 41: Szenarienvergleich Item 4.

Abbildung 42: Szenarienvergleich mit Differenzierung nach "Zusatz-Weiterbildung Palliativmedizin" Item 4

\section{Tabellenverzeichnis}

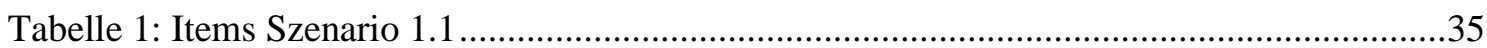

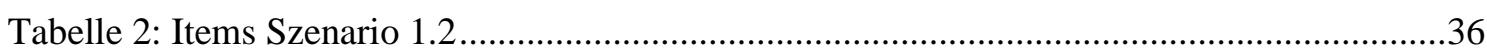

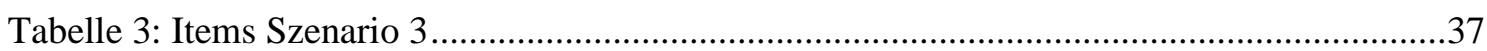

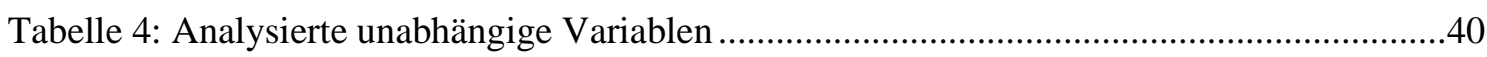

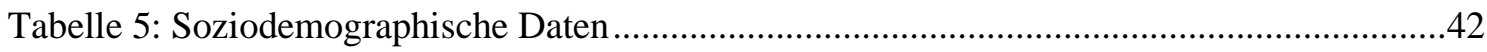

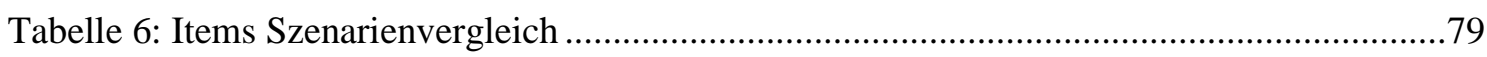




\section{Abkürzungsverzeichnis}

AEM

ALS

AWMF

CCC

DGN

EAPC

SOP
Akademie für Ethik in der Medizin

Amyotrophe Lateralsklerose

Arbeitsgemeinschaft der Wissenschaftlichen Medizinischen Fachgesellschaften

Comprehensive Cancer Center

Deutsche Gesellschaft für Neurologie

European Association for Palliative Care

Standard Operating Procedure 


\section{$1 \quad$ Einleitung}

Die Gründung des St. Christopher`s Hospice in London im Jahre 1967 durch Cicely Saunders wird zumeist als Ursprung der modernen Palliativmedizin betrachtet. Vor dem Hintergrund einer in erster Linie technisch ausgerichteten Medizin, entwickelte sich zu dieser Zeit zunehmend ein Bewusstsein für die Wichtigkeit der Versorgung Sterbender. In Deutschland wurden in den 1980er Jahren die erste Palliativstation und das erste Hospiz eröffnet (Husebø und Mathis 2017; Maier und Sitte 2016).

Im Zentrum palliativmedizinischen Handelns steht die Erhaltung oder Verbesserung der Lebensqualität von PatientInnen mit einer nicht heilbaren Erkrankung. Palliative Versorgung ist interdisziplinär und multiprofessionell angelegt und verfolgt einen holistischen Ansatz, der körperliche, psychische, spirituelle und soziale Bedürfnisse und Präferenzen berücksichtigt (AWMF 2018).

Im Fokus der modernen Palliativmedizin stehen vor allem onkologische PatientInnen. Etwa $90 \%$ der palliativmedizinisch betreuten PatientInnen weisen eine Krebserkrankung auf (vgl. Alt-Epping et al. 2008; Husebø und Mathis 2017). Auch die aktuelle S3-Leitlinie Palliativmedizin bezieht sich explizit nur auf PatientInnen mit einer nicht heilbaren Krebserkrankung (AWMF 2018). Prinzipiell ist das Angebot palliativmedizinischer Maßnahmen jedoch unabhängig von der Grunderkrankung und die Notwendigkeit der Ausweitung palliativmedizinischer Versorgung auf nicht-onkologische PatientInnen wird in der medizinischen und medizinethischen Literatur immer wieder betont (Bausewein 2005; Borasio und Volkenandt 2007; Husebø und Mathis 2017).

In der vorliegenden Arbeit steht die Palliative Sedierung als eine Maßnahme palliativmedizinischer Versorgung im Fokus. In der deutschen Übersetzung der Rahmenleitlinie der European Association for Palliative Care (EAPC) wird die Palliative Sedierung beschrieben als der

„[. . . überwachte Einsatz von Medikamenten mit dem Ziel einer verminderten oder aufgehobenen Bewusstseinslage (Bewusstlosigkeit), um die Symptomlast in anderweitig therapierefraktären Situationen in einer für Patienten, Angehörige und Mitarbeiter ethisch akzeptablen Weise zu reduzieren“ (Alt-Epping et al. 2010).

Bis in die 2000er Jahre wurde für das beschriebene Vorgehen zumeist der Begriff „Terminale Sedierung“"verwendet, der von Enck (1991) geprägt wurde. Auch benutzen einige 
AutorInnen den Terminus „Sedierung am Lebensende“ (Müller-Busch 2004). In den letzten Jahren hat sich jedoch zunehmend der Begriff der Palliativen Sedierung durchgesetzt, der unter anderem in der aktuellen S3-Leitlinie Palliativmedizin verwendet wird (AWMF 2018). Der Vorteil dieses Begriffs besteht darin, dass er nicht auf eine konkrete Krankheitsphase hindeutet, sondern die Symptomlinderung in den Vordergrund stellt (Weber et al. 2005). Hingegen kritisiert Rothärmel (2004), dass der Begriff auch vorübergehende Sedierungspraktiken, beispielsweise im Rahmen von Intensivbehandlungen, einschließe und sich nicht konkret auf terminal erkrankte PatientInnen beziehe. Im Rahmen dieser Arbeit wird dennoch der Begriff der Palliativen Sedierung verwendet und die oben aufgeführte Definition zugrunde gelegt.

In der vorliegenden Arbeit steht die Palliative Sedierung bei PatientInnen mit Amyotropher Lateralsklerose (ALS) im Fokus. Im Folgenden wird zunächst der aktuelle Stand der Forschung erörtert. Dabei wird die Palliative Sedierung mit ihren Formen, Zielen und Voraussetzungen beschrieben. In diesem Zuge sollen sowohl rechtliche als auch medizinethische Grundlagen thematisiert und aktuelle Richtlinien zum Einsatz der Palliativen Sedierung dargestellt werden. Zudem werden die in der medizinischen und medizinethischen Diskussion bestehenden Kontroversen um die Thematik aufgegriffen. Anschließend wird das Krankheitsbild der ALS beschrieben und daran anknüpfend werden medizinethische Überlegungen zur Palliativen Sedierung bei ALS angestellt.

Um die medizinethische Theorie mit der klinischen Praxis zu verknüpfen, wurde im Rahmen dieser Arbeit eine Fragebogenstudie mit weiterbildungsermächtigten ÄrztInnen aus dem Bereich der Neurologie und der Palliativmedizin durchgeführt. Die Umfrage soll Hinweise darauf liefern, ob eine Palliative Sedierung bei ALS im klinischen Alltag zum Einsatz kommt und unter welchen Bedingungen die Teilnehmenden einer Palliativen Sedierung bei ALS-PatientInnen zustimmen würden.

Im empirischen Teil der Arbeit werden zunächst die sich aus den vorangegangenen Kapiteln ergebenden Forschungsfragen erörtert. Daraufhin werden Material und Methoden der Fragebogenstudie beschrieben. Anschließend werden die Ergebnisse der Fragebogenstudie dargestellt und im Hinblick auf die Forschungsfragen diskutiert. 


\subsection{Stand der Forschung}

\subsubsection{Formen und Ziele der Palliativen Sedierung}

Hinter dem Begriff der Palliativen Sedierung verbirgt sich zumeist kein einheitliches, standardisiertes Vorgehen, sondern vielmehr verschiedene Formen und Stufen der Bewusstseinsdämpfung. Das Ziel ist dabei stets die Linderung von schweren Leidenszuständen, doch die Tiefe und Dauer der Sedierung und damit auch die Art und Dosis der Medikation müssen individuell angepasst werden (Cellarius 2008; Cherny et al. 2009).

Beispielsweise beschreiben Neitzke et al. (2010) zwei Sedierungsstufen, die sich hinsichtlich ihrer Indikation, ihrer Ziele und ihrer medizinethischen Bewertung unterscheiden. Eine Sedierung der Stufe I sei prinzipiell flach und reversibel angelegt, sodass die Betroffenen von belastenden Symptomen abgeschirmt sind, jedoch wiedererweckt werden können. In Sedierungspausen könne die Situation dann sowohl von den PatientInnen als auch von ÄrztInnen und Pflegenden neu evaluiert werden. Die Stufe I könne wiederum in eine Stufe Ia mit intermittierender Sedierung und eine Stufe Ib mit kontinuierlicher, aber flacher Sedierung unterteilt werden (Neitzke et al. 2010).

Eine Sedierung der Stufe II sei hingegen kontinuierlich, tief und irreversibel angelegt und verfolge meist das Ziel, die Betroffenen bis zum Tod von belastenden Symptomen zu befreien. Die Autoren merken an, dass aufgrund der Endgültigkeit einer solchen Maßnahme - außer in Notfallsituationen - zunächst eine Sedierung der Stufe I erfolgen sollte. Nur wenn die Beschwerden dadurch nicht in ausreichendem Maße gelindert werden können, sollte eine Sedierung der Stufe II angeboten werden (Neitzke et al. 2010). Auch Cherny (2014) merkt an, dass stets eine flache Sedierung bevorzugt werden sollte, wenn dadurch eine adäquate Symptomlinderung gewährleistet werden kann: „For some patients, a state of 'conscious sedation', in which the ability to respond to verbal stimuli is retained, may provide adequate relief without total loss of interactive function".

Ebenso wie die Wahl der Sedierungsform, hänge auch die Entscheidung für oder gegen begleitende Maßnahmen während der Sedierung vom Therapieziel ab. Hinsichtlich des Monitorings der Vitalfunktionen empfiehlt Cherny (2014) keinen routinemäßigen Einsatz bei PatientInnen, die sich bereits unmittelbar in der Sterbephase befinden. In diesen Fällen sollten primär klinische Anzeichen für Unwohlsein - wie beispielsweise Tachypnoe erfasst werden und eine entsprechende Dosisanpassung der sedierenden Medikamente erfolgen. Befinden sich PatientInnen nicht in der unmittelbaren Sterbephase oder ist die 
Sedierung intermittierend angelegt, könne hingegen ein regelmäßiges Monitoring von Parametern wie Blutdruck, Herzfrequenz und Sauerstoffsättigung durchgeführt werden, um eine Über- oder Unterdosierung zu vermeiden (Cherny 2014).

Weitere Begleitmaßnahmen umfassen künstliche Nahrungs- und Flüssigkeitszufuhr, Beatmung oder Medikation. Insbesondere der Abbruch lebenserhaltender Maßnahmen unter Sedierung wird in der medizinethischen Literatur kontrovers diskutiert (Cherny et al. 2009; Neitzke et al. 2010; Simon 2008). In jedem Fall fortgesetzt werden müssen jedoch auch unter Sedierung grundlegende pflegerische Maßnahme, wie Körperpflege und menschliche Zuwendung (Neitzke et al. 2010). In der Rahmenleitlinie der EAPC wird betont, dass Entscheidungen über Nahrungs- und Flüssigkeitszufuhr stets im Kontext der individuellen Situation der PatientInnen getroffen werden müssen, da diese Maßnahmen einerseits Leiden lindern können, andererseits aber auch negative Begleiterscheinungen hervorrufen können (Cherny et al. 2009).

\subsubsection{Normative Voraussetzungen der Palliativen Sedierung}

Der Durchführung der Palliativen Sedierung liegen bestimmte Rahmenbedingungen und normative Überlegungen zugrunde. Einige dieser normativen Voraussetzungen gelten für jeglichen medizinischen Eingriff, doch birgt eine Maßnahme wie die Palliative Sedierung auch spezifische Herausforderungen. Im Folgenden soll der normative Rahmen der Palliativen Sedierung dargestellt werden. Dabei wird zunächst das Zwei-Säulen-Modell aus ärztlicher Indikation und Einwilligung beschrieben, das grundsätzlich für alle medizinischen Maßnahmen gültig ist. Anschließend werden die spezifischen rechtlichen und medizinethischen Grundlagen der Palliativen Sedierung erörtert.

\subsubsection{Medizinische Indikation und informierte Einwilligung}

Ebenso wie jede andere medizinische Maßnahme, benötigt die Durchführung der Palliativen Sedierung einerseits eine medizinische Indikation und andererseits die informierte Einwilligung der PatientInnen (Zwei-Säulen-Modell) (Simon 2004). Die medizinische Indikation wird von ÄrztInnen gestellt und bezieht Wissen über Krankheitsverläufe, Therapieziel, Wirkung verschiedener Therapien und die individuelle Situation der Betroffenen ein. Die behandelnden ÄrztInnen haben demnach die Aufgabe, Vor- und Nachteile einer Maßnahme abzuwägen und deren Nutzen im Hinblick auf das Therapieziel zu bewerten. Eine Maßnahme kann beispielsweise als medizinisch nicht indiziert bewertet werden, wenn die Nachteile stark überwiegen oder wenn sie nicht zum Erreichen des 
festgelegten Therapieziels beiträgt. Eine medizinisch nicht indizierte Behandlung darf PatientInnen nicht angeboten werden (Simon 2004). Dabei ist jedoch zu berücksichtigen, dass das Therapieziel in der Regel nicht einseitig durch die ÄrztInnen, sondern in Kommunikation mit den PatientInnen bestimmt wird. Zur Indikation der Palliativen Sedierung heißt es im Rahmenkonzept der EAPC:

„Sedation is potentially indicated for patients with intolerable distress due to physical symptoms, when there is a lack of other methods for palliation within an acceptable time frame and without unacceptable adverse effects (refractoriness)“ (Cherny et al. 2009).

Palliative Sedierung gilt also grundsätzlich nicht als Mittel der ersten Wahl, sondern als Möglichkeit der Linderung von unerträglichem Leid, wenn konventionelle Formen der Behandlung nicht erfolgreich sind. Diese Therapierefraktärität der Symptome wird in der medizinethischen Literatur wiederholt als eine Voraussetzung für die Durchführung einer Palliativen Sedierung genannt (Cherny et al. 2009; Neitzke et al. 2010).

Braun et al. (2003) fordern als Voraussetzung für die Durchführung einer Palliativen Sedierung neben der Therapierefraktärität der Symptome das Vorliegen einer Patientenverfügung, die den Verzicht auf Wiederbelebung enthält, zudem das Vorhandensein einer zum Tode führenden Krankheit und schließlich eine Lebenserwartung von nur einigen Tagen. Diese Aspekte werden jedoch in der medizinethischen Literatur kontrovers diskutiert (Cellarius 2008; Cherny et al. 2009).

Prinzipiell kann die Indikation für eine Palliative Sedierung sowohl aufgrund körperlicher als auch aufgrund psychischer Symptome gestellt werden. Psychische Symptome, die eine Palliative Sedierung rechtfertigen können, sind beispielsweise existentielle Not, Angst, Panik oder Depression. Jedoch erscheint die Legitimation bei psychischen Symptomen deutlich schwieriger, da die Refraktärität und die Alternativlosigkeit der Therapie oft nur schwer festgestellt werden können (Neitzke et al. 2010). Auch die Rahmenleitlinie der EAPC weist darauf hin, dass es keinen Konsens bezüglich Palliativer Sedierung bei nicht-körperlicher Symptomatik gebe, doch dass ein solcher Einsatz unter besonderen Vorsichtsmaßnahmen erwogen werden könne (Cherny et al. 2009).

Die medizinische Indikation allein legitimiert die Durchführung einer Maßnahme jedoch noch nicht, denn in einem zweiten Schritt müssen die PatientInnen der Behandlung zustimmen. Eine solche Zustimmung ist nur wirksam, wenn die Betroffenen einerseits ausreichend aufgeklärt und andererseits einwilligungsfähig sind (Simon 2004). Zudem kann 
eine Einwilligung jederzeit wiederrufen werden. Für eine umfassende Aufklärung ist es nicht ausreichend, die notwendigen Informationen schriftlich zur Verfügung zu stellen oder sie den PatientInnen lediglich vorzutragen. Vielmehr muss sichergestellt werden, dass die Betroffenen ihre Diagnose, die Durchführung der Behandlung und die Vor- und Nachteile verschiedener Behandlungsoptionen auch verstanden haben und somit eine informierte Entscheidung treffen können (Simon 2015).

Als einwilligungsfähig gelten PatientInnen, wenn sie über ausreichend Urteils- und Einsichtsfähigkeit verfügen, um die Art der Behandlung und ihre möglichen Folgen einschätzen zu können. Diese Anforderung schließt Minderjährige, psychisch Kranke und demente PatientInnen nicht grundsätzlich aus. Jedoch ist die Beurteilung der Einwilligungsfähigkeit nicht immer einfach und muss im Einzelfall genau abgewogen werden. Es ist beispielsweise möglich, dass PatientInnen einfachere Maßnahmen verstehen und diesbezüglich über Einwilligungsfähigkeit verfügen, doch kompliziertere Therapieoptionen nicht vollständig erfassen können (Simon 2015).

Vollständig aufgeklärte und einwilligungsfähige PatientInnen haben also im Sinne eines Rechts auf Selbstbestimmung die Möglichkeit, Behandlungen abzulehnen. Das Recht auf Selbstbestimmung bleibt auch dann bestehen, wenn im Nachhinein einer Entscheidung die Einwilligungsfähigkeit nicht mehr gegeben ist. Früher getroffene Entscheidungen beispielsweise zum Einsatz lebenserhaltender Maßnahmen - sind weiterhin bindend, auch wenn PatientInnen aktuell nicht einwilligungsfähig sind (Simon 2015).

\subsubsection{Rechtliche Grundlagen}

Das Grundgesetz garantiert in Artikel 1 den Schutz der Menschenwürde und der Menschenrechte, in Artikel 2 das Recht auf freie Entfaltung der Persönlichkeit, auf Leben und auf körperliche Unversehrtheit. Damit soll einerseits ein Schutz der PatientInnen vor nicht gewünschten medizinischen Eingriffen gewährleistet werden. ÄrztInnen dürfen keine medizinische Maßnahme gegen den Willen der Betroffenen durchführen, was auch die Einleitung oder Aufrechterhaltung künstlicher Beatmung und Ernährung betrifft (Nationaler Ethikrat 2006). Die Durchführung einer medizinischen Maßnahme gegen den expliziten Wunsch der PatientInnen erfüllt den Tatbestand der Körperverletzung nach $§ 223$ Abs. 1 StGB (Nationaler Ethikrat 2006). Andererseits wird jedem Menschen das Recht zugesprochen, das eigene Leben und somit auch das Lebensende selbstbestimmt zu gestalten. Mit dem Schutz der Menschenwürde wird deshalb oft auch ein Recht auf ein 
würdiges Sterben und auf Selbstbestimmung in der Sterbephase verbunden (Kreß 2004; Nationaler Ethikrat 2006).

In diesem Kontext erscheint eine Erläuterung der verschiedenen Formen der Sterbehilfe sinnvoll, da die Palliative Sedierung zuweilen mit einer Tötung auf Verlangen gleichgesetzt wird. Eine Tötung auf Verlangen (früher: aktive Sterbehilfe) ist in Deutschland gesetzlich verboten. Tötung auf Verlangen bezeichnet die gezielte Herbeiführung des Todes und ist nach $\S 216$ Abs.1 StGB strafbar, auch wenn sie auf den Willen des Betroffenen hin erfolgt.

Wird die Palliative Sedierung entsprechend der oben aufgeführten Definition angewendet, ist sie nicht mit einer Tötung auf Verlangen gleichzusetzen, da Palliative Sedierung stets eine Symptomlinderung bewirken soll und nicht die Verkürzung des Lebens zum Ziel hat (Simon 2006). Gleichwohl muss erwähnt werden, dass eine tiefe kontinuierliche Sedierung - insbesondere wenn gleichzeitig lebenserhaltende Maßnahmen beendet werden - zu einem früheren Eintritt des Todes führen kann. Auch ist es nicht ausgeschlossen, dass die zur Palliativen Sedierung eingesetzten Medikamente absichtlich so dosiert und kombiniert werden, dass ein zeitnaher Todeseintritt zu erwarten ist. Dieses Vorgehen entspricht einer Tötung auf Verlangen. Lipuma (2013) argumentiert, dass der Einsatz einer tiefen kontinuierlichen Sedierung bis zum Tod als Ausweg gebraucht wird, um sowohl eine Tötung auf Verlangen zumindest vordergründig zu umgehen, als auch unerträgliches und therapierefraktäres Leiden zu beenden.

Unter dem Begriff der geschäftsmäßigen Suizidhilfe werden Handlungen zusammengefasst, die auf Wiederholung ausgelegt sind und die Selbsttötung anderer fördern oder erleichtern. Eine solche systematische Beihilfe zum Suizid wurde im Jahr 2015 nach $§ 217$ Abs. 1 StGB strafbar, während einzelne Fälle, in denen aus Mitleid die Selbsttötung erleichtert oder nicht verhindert wird aber keine Wiederholungsabsicht besteht, straffrei blieben (Simon 2017). Im Februar 2020 urteilte jedoch das Bundesverfassungsgericht, dass dieses Verbot gegen das Grundgesetz verstoße, da jeder Mensch die Freiheit haben müsse, sowohl sich das Leben zu nehmen als auch dabei Hilfe in Anspruch zu nehmen (Bundesverfassungsgericht 2020).

Zulässig ist weiterhin ein Behandlungsabbruch (früher: passive Sterbehilfe), unter dem man das Unterlassen, Begrenzen oder Beenden lebenserhaltende Maßnahmen - in Übereinstimmung mit dem Willen der PatientInnen - versteht (HRR-Strafrecht 2010). Sowohl 
unter Laien als auch unter medizinischem Personal scheinen jedoch noch immer Unsicherheiten bezüglich der Rechtmäßigkeit eines Behandlungsabbruchs zu bestehen (Sitte et al. 2016). Eine von van Oorschot et al. (2005) durchgeführte Befragung von InternistInnen, AnästhesistInnen und AllgemeinmedizinerInnen zeigte, dass etwa $10 \%$ der Befragten den Verzicht auf künstliche Beatmung als aktive Sterbehilfe klassifizieren würden. Die Beendigung einer bereits bestehenden künstlichen Beatmung wurde sogar von fast $40 \%$ der aktiven Sterbehilfe zugeordnet.

In der aktuellen S3-Leitlinie Palliativmedizin wird in diesem Kontext der Begriff „Sterben zulassen“" verwendet und das Vorgehen explizit als erlaubt und in bestimmten Fällen als ethisch und rechtlich geboten bezeichnet (AWMF 2018).

Die Palliative Sedierung ist folglich selbst nicht als Behandlungsabbruch einzustufen, jedoch entstehen Berührungspunkte, wenn eine Palliative Sedierung und gleichzeitig die Beendigung lebenserhaltender Maßnahmen gewünscht werden. Rechtlich erscheint dies möglich, wenn sowohl medizinische Indikation und Einwilligung des Betroffenen zur Sedierung als auch der explizite Wunsch nach Behandlungsabbruch bestehen. Die Legitimität dieses Vorgehens wird aufgrund der möglicherweise resultierenden Lebensverkürzung in der medizinethischen Literatur jedoch kontrovers diskutiert (Cherny et al. 2009; Davis und Ford 2005; Lipuma 2013; Neitzke et al. 2010).

Schließlich bezeichnet der Begriff der Symptomlinderung (früher: indirekte Sterbehilfe) eine ungewollte Verkürzung des Lebens als Nebenwirkung von Maßnahmen, die die Leidenslinderung zum Ziel haben. Solche Maßnahmen umfassen beispielsweise Schmerzmedikation oder Sedierung. Somit kann Palliative Sedierung am ehesten einer solchen Symptomlinderung zugeordnet werden (Beck 2004). Eine Lebensverkürzung als ungewollte Folge einer Sedierung kann zunächst strafrechtlich und medizinethisch problematisch erscheinen und die Grenzen zur Tötung auf Verlangen verschwimmen lassen.

Verschiedene Urteile deutscher Gerichte geben der Leidenslinderung jedoch Vorrang vor einer reinen Verlängerung des Lebens. Unter der Voraussetzung, dass die Palliative Sedierung nicht mit dem Ziel der Lebensverkürzung eingesetzt wird, rechtfertigen weder das Strafgesetzbuch noch das Grundgesetz einen Zwang zur Lebensverlängerung unter starken Schmerzen oder anderen unerträglichen Belastungen (Simon 2006). Dazu betont auch der Nationale Ethikrat:

„Denn wenn diese lebensverkürzende Schmerzlinderung [...] verboten wäre, liefe das auf eine Pflicht zum Weiterleben unter heftigsten Schmerzen hinaus. Das aber wäre 
nicht nur ethisch kaum zu rechtfertigen, sondern stünde auch mit den Prämissen unserer Rechtsordnung nicht in Einklang, die der Selbstbestimmung des Einzelnen hohen Rang einräumt und insbesondere mit der Garantie der Menschenwürde eine Verpflichtung zur Humanität eingegangen ist“" (Nationaler Ethikrat 2006).

\subsubsection{Medizinethische Grundlagen}

Ergänzend zu den genannten rechtlichen Aspekten, soll nun die prinzipienorientierte Medizinethik nach Beauchamp und Childress (2001) als eine mögliche medizinethische Grundlage der Palliativen Sedierung beschrieben werden. Diese wurde in den 1970er Jahren begründet und ist insbesondere deshalb von großer Bedeutung, weil aus den klassischen philosophischen Theorien, die einem grundlegenden Moralprinzip folgen, keine konsensfähigen Anhaltspunkte für die medizinische Praxis abgeleitet werden konnten. An die Stelle eines übergreifenden Moralprinzips treten hier vier gleichwertige Prinzipien, die sich an weithin akzeptierten Moralvorstellungen orientieren. Diese Prinzipien bieten einen Orientierungsrahmen für medizinisches Handeln, der jedoch Spielraum für individuelle Anpassungen lässt und somit keine konkreten Handlungsanweisungen enthält (Beauchamp und Childress 2001; Marckmann 2015).

Als eine der vier Grundlagen medizinischen Handelns beschreiben Beauchamp und Childress (2001) den Respekt vor der Autonomie der PatientInnen. Notwendige Bedingungen für die Autonomie einer Person seien einerseits Handlungskompetenz und andererseits Unabhängigkeit von kontrollierenden äußeren Einflüssen. Autonome Personen handeln also frei und in Übereinstimmung mit ihren selbst gewählten Plänen, ohne dass ihr Handeln durch äußere Einflüsse beeinträchtigt wird.

Das Prinzip der Autonomie wendet sich gegen einen ärztlichen Paternalismus und eine wenn auch gut gemeinte - Bevormundung von PatientInnen. Somit sollten ÄrztInnen von jeglicher Manipulation des PatientInnenwillens Abstand nehmen und zudem die Entscheidungsfindung aktiv unterstützen. Autonomie wird also nicht lediglich als Abwehrrecht gegen ärztliche Behandlungsvorschläge definiert. Einer umfassenden und möglichst objektiven Aufklärung unter Einbeziehung der individuellen Situation der Betroffenen kommt dabei eine große Bedeutung zu (Beauchamp und Childress 2001).

Weiterhin wird das Prinzip des Nichtschadens beschrieben, welches zunächst einmal nur besagt, dass PatientInnen kein Schaden zugefügt werden darf. Doch was auf den ersten Blick selbstverständlich erscheint, ist tatsächlich deutlich komplizierter, da die meisten 
medizinischen Eingriffe einen Schaden mit sich bringen, der jedoch zugunsten eines erwarteten Vorteils in Kauf genommen wird. Auch deshalb wird das Prinzip des Nichtschadens explizit vom Prinzip des Wohltuns abgegrenzt, welches eine aktive Förderung des Wohlergehens anderer beinhaltet (Beauchamp und Childress 2001).

Schließlich wird das Prinzip der Gerechtigkeit genannt, welches auf dem Grundsatz basiert, dass gleiche Fälle gleich und ungleiche Fälle ungleich behandelt werden sollten. Probleme entstehen jedoch, wenn konkretisiert werden muss, inwieweit PatientInnen gleich behandelt werden sollen und welche Unterschiede eine unterschiedliche Behandlung rechtfertigen. Beispielsweise können gleiche medizinische Leistungen entweder allen Einwohnern eines Landes oder nur Personen eines bestimmten Alters oder nur Personen mit einem bestimmten Bedarf zur Verfügung gestellt werden. Welche Aspekte relevant sind, um Fälle als gleich oder ungleich zu definieren, wird nicht abschließend geklärt (Beauchamp und Childress 2001).

Die vier geschilderten Prinzipien stehen gleichwertig nebeneinander und sollten jeweils so weit verfolgt werden, bis sie miteinander in Konflikt geraten. Wie bereits angesprochen, entsteht ein solcher Konflikt regelmäßig zwischen dem Prinzip des Wohltuns und dem Prinzip des Nichtschadens. Im Kontext der Palliativen Sedierung muss beispielsweise die Symptomlinderung gegen einen Verlust der Kommunikationsfähigkeit und das Risiko der Lebenszeitverkürzung abgewogen werden. Ebenso können Konflikte hinsichtlich des Autonomieprinzips entstehen, wenn PatientInnen eine Palliative Sedierung wünschen, diese von den behandelnden ÄrztInnen jedoch im Hinblick auf das Prinzip des Nichtschadens abgelehnt wird.

Die Autoren merken zudem an, dass die vier Prinzipien lediglich einen groben Orientierungsrahmen bieten und nicht dazu geeignet seien, konkrete Lösungen für jegliche Konfliktsituation anzubieten. Zur Anwendung im klinischen Alltag müssten die Prinzipien deshalb spezifiziert und mit Inhalt gefüllt werden (Beauchamp und Childress 2001).

\subsubsection{Bestehende Richtlinien zur Palliativen Sedierung}

Der Einsatz der Palliativen Sedierung ist in Deutschland bisher nicht umfassend formal geregelt. Wie bereits beschrieben, besteht eine große Variabilität hinsichtlich der genauen Ausgestaltung (Art und Dosis der Medikation, Monitoring, Sedierungstiefe). Es wurden jedoch einige Empfehlungen verfasst, die eine grundlegende Orientierung bieten. 
Die AG Ethik am Lebensende der Akademie für Ethik in der Medizin (AEM) hat im Jahr 2010 Empfehlungen zur Sedierung am Lebensende herausgegeben. Sie verfolgt das Ziel, die öffentliche Diskussion zur Palliativen Sedierung voranzubringen und ebenso Kritik und Ängste bezüglich eines potenziellen Missbrauchs aufzugreifen (Neitzke et al. 2010). Zudem hat die EAPC - deren Untergesellschaft die Deutsche Gesellschaft für Palliativmedizin ist - im Jahr 2009 eine 10-Punkte-Rahmenleitlinie zum Einsatz der Palliativen Sedierung veröffentlicht, die den damaligen Stand der Forschung und des medizinethischen Diskurses aufgreift. Diese Rahmenleitlinie soll nicht als feststehendes Regelwerk angesehen werden, sondern vielmehr einen Rahmen für die klinische Praxis bieten und Anhaltspunkte für die Ausarbeitung konkreter Richtlinien auf lokaler Ebene liefern (Cherny et al. 2009).

Basierend auf verschiedenen internationalen Empfehlungen, wurde im Jahr 2017 eine Standard Operating Procedure (SOP) „Palliative Sedierung“ veröffentlicht, die von der Arbeitsgemeinschaft Palliativmedizin des von der Deutschen Krebshilfe geförderten Comprehensive Cancer Center-Netzwerks (CCC-Netzwerk) erstellt wurde. Die SOP wurde im März 2019 aktualisiert und stellt in Form von Stichpunkten und Flow-Charts Empfehlungen zu Indikationsstellung, Durchführung, Formen und Monitoring der Palliativen Sedierung zur Verfügung.

Die Rahmenleitlinie der EAPC adressiert zunächst die frühzeitige Thematisierung und Planung der Palliativen Sedierung. Es wird empfohlen, die Maßnahme möglichst früh mit lebensbedrohlich Erkrankten und deren Angehörigen zu besprechen. Dies sei insbesondere dann sinnvoll, wenn der Eintritt in die Sterbephase oder eine akute Verschlechterung der Situation absehbar sind. Es sollten dabei die Ziele der weiteren Behandlung, die persönlichen Prioritäten der Betroffenen und konkrete Pläne für das Vorgehen in bestimmten Situationen angesprochen werden. Beispielsweise sollten Maßnahmen wie Reanimation und künstliche Nahrungs- und Flüssigkeitszufuhr thematisiert werden, sodass in einer Notfallsituation Klarheit für alle Beteiligten besteht. Die getroffenen Entscheidungen sollten anschließend dokumentiert werden (Cherny et al. 2009).

Weiterhin wird in den Veröffentlichungen die Indikationsstellung für eine Palliative Sedierung erörtert. Die Therapierefraktärität der Symptome wird in allen drei Veröffentlichungen als Voraussetzung für die Durchführung einer Palliativen Sedierung hervorgehoben. Laut der SOP „Palliative Sedierung“ sollte in die Indikationsstellung zudem eine multiprofessionelle Objektivierung des Leidensdrucks, eine Abschätzung der Dynamik 
und eine Reevaluation alternativer Therapien einfließen. Eine darauf basierende Entscheidung für oder gegen eine Palliativen Sedierung sollte dokumentiert werden (Oechsle et al. 2017). Das Ziel der Palliativen Sedierung müsse dabei stets die Leidenslinderung und nie die absichtliche Verkürzung der Lebenszeit sein (Cherny et al. 2009; Neitzke et al. 2010).

Zudem werden in den Veröffentlichungen sowohl körperliche als auch psychische Symptome als Indikation für eine Palliative Sedierung genannt. Es wird jedoch darauf hingewiesen, dass die Therapierefraktärität bei psychischen Symptomen deutlich schwieriger beurteilbar sei als bei körperlichen Leiden. Die Entscheidung für eine Palliative Sedierung bei psychischen Symptomen müsse deshalb besonders sorgfältig überlegt sein und sollte ÄrztInnen einbeziehen, die Erfahrungen mit psychologischer Betreuung aufweisen (Cherny et al. 2009; Neitzke et al. 2010). In der SOP „Palliative Sedierung“ wird in diesem Kontext noch einmal explizit die Notwendigkeit eines multiprofessionellen Ansatzes hervorgehoben und zudem darauf hingewiesen, dass die Entscheidungsfähigkeit der PatientInnen geprüft werden müsse (Oechsle et al. 2017).

Abgesehen von Notfallsituationen oder Situationen mit sehr hohem Leidensdruck, wird eine tiefe und kontinuierliche Sedierungen eher für die Finalphase schwerer Erkrankungen empfohlen, während flachere Formen auch in früheren Krankheitsphasen zur Anwendung kommen können. Durch den initialen Versuch einer flachen intermittierenden Sedierung, könne die Interaktionsfähigkeit der Betroffenen zumindest teilweise erhalten bleiben. Sedierungspausen bieten zudem die Möglichkeit der Reevaluation und der Kommunikation mit Angehörigen und medizinischem Personal. Reicht eine solche flache Sedierung nicht aus, um die Symptomlast in angemessener Weise zu senken, sollte eine tiefere und kontinuierliche Sedierung erfolgen (Cherny et al. 2009; Neitzke et al. 2010; Oechsle et al. 2017).

Überdies wird der kontroverse Aspekt der lebenserhaltenden Maßnahmen während einer Palliativen Sedierung adressiert. Neitzke et al. (2010) betonen, dass eine Sedierung der Stufe I prinzipiell reversibel angelegt sei und deshalb sämtliche Begleitmaßnahmen fortgesetzt oder gegebenenfalls neu begonnen werden müssen. Sollten Betroffene diese Maßnahmen ablehnen, müsse die Entscheidung für eine Sedierung durch die ÄrztInnen neu überdacht werden. Hingegen seien lebensverlängernde Verfahren unter einer Stufe II Sedierung laut Neitzke et al. (2010) nicht indiziert, da in diesen Fällen Lebensverlängerung explizit nicht mehr Therapieziel ist. 
Cherny et al. (2009) vertreten hingegen die Meinung, dass die Entscheidung für oder gegen lebenserhaltende Maßnahmen unabhängig von der Entscheidung für oder gegen eine Palliative Sedierung erfolgen müsse und geben umfassende Vorgaben für Monitoring und weitere Begleitmaßnahmen unter der Sedierung. Ein regelmäßiges Monitoring verfolge das Ziel, Sedierungstiefe und Symptomlast festzustellen und die Medikamentendosis entsprechend anzupassen (Cherny et al. 2009; Neitzke et al. 2010; Oechsle et al. 2017).

Auch der Aspekt der Qualitätssicherung wird in den Veröffentlichungen erörtert. Laut Neitzke et al. (2010) sollte für jede Sedierungsstufe evaluiert werden, ob die notwendigen Rahmenbedingungen - wie ausreichend geschultes Personal und apparative Ausstattung - gegeben sind. Die Einleitung der Palliativen Sedierung sollte möglichst von erfahrenen ÄrztInnen und unter Einbeziehung weiteren medizinischen Personals geschehen.

Die Entscheidung für eine Palliative Sedierung stellt auch für die Angehörigen oft eine große Belastung dar. Insbesondere mit dem Beginn einer Stufe II Sedierung ist eine Kommunikation nicht mehr möglich, sodass sich Angehörige nicht mehr versichern können, ob die getroffenen Entscheidungen vom Erkrankten weiterhin befürwortet werden. Oechsle et al. (2017) weisen darauf hin, dass auch die Angehörigen über die aktuellen Therapiemöglichkeiten, über Vorteile und über Grenzen der Palliativen Sedierung informiert werden sollten.

Cherny et al. (2009) betonen, dass es den Angehörigen ermöglicht werden sollte, kontinuierlich anwesend zu sein, sofern dies von den Betroffenen gewünscht wird. Es wird unter anderem empfohlen, dass Übernachtungsmöglichkeiten zur Verfügung gestellt werden und die Umgebung so gestaltet wird, dass Angehörige in einer möglichst angenehmen Atmosphäre Abschied nehmen können. Ebenfalls sollten sie regelmäßig über den Zustand der PatientInnen informiert und emotional unterstützt werden (Cherny et al. 2009).

Aus den geschilderten Anforderungen wird deutlich, dass auch das Behandlungsteam starken psychischen Belastungen ausgesetzt ist. In der EAPC-Leitlinie wird darauf hingewiesen, dass solche Belastungen insbesondere dann entstehen, wenn die Angemessenheit der Palliativen Sedierung als fraglich angesehen wird (Cherny et al. 2009). Insbesondere im Hinblick auf eine empfundene Nähe der tiefen kontinuierlichen Sedierung zu einer Tötung auf Verlangen, ist es denkbar, dass Behandler vor einem ethischen Konflikt stehen. Deshalb sollten möglichst alle Beteiligten die getroffenen Entscheidungen nachvollziehen können und es sollte eine regelmäßige Reflexion der Behandlungsverläufe 
stattfinden. Es könne außerdem erwogen werden, die Angehörigen in eine solche Evaluation einzubeziehen, um künftige Behandlungsstrategien anzupassen (Cherny et al. 2009; Neitzke et al. 2010).

Die beschriebenen Empfehlungen beziehen sich primär auf die Anwendung der Palliativen Sedierung in Deutschland (Empfehlungen der AG Ethik am Lebensende der AEM, SOP „Palliative Sedierung“") und Europa (EAPC-Rahmenleitlinie). Schildmann und Schildmann (2014) haben im Rahmen einer systematischen Übersichtsarbeit neun Leitlinien auf internationaler Ebene verglichen. Neben der EAPC-Rahmenleitlinie wurden Empfehlungen aus den USA, Kanada, Japan und den Niederlanden und eine internationale Leitlinie eingeschlossen.

Es zeigen sich zum Teil ähnliche Herangehensweisen und Konflikte wie im deutschen und europäischen Kontext. Beispielsweise werden in allen Leitlinien unerträgliche, therapierefraktäre Belastungen als Indikation für die Palliative Sedierung genannt. Auch wird der Einsatz bei psychischer Symptomatik als besonders schwierig eingestuft (Schildmann und Schildmann 2014). Die bereits beschriebenen Konflikte um den Abbruch lebenserhaltender Maßnahme spiegeln sich ebenfalls beim Vergleich der verschiedenen Leitlinien wider. In vier Leitlinien wird eine Patientenverfügung mit Verzicht auf Wiederbelebung als Voraussetzung für eine Palliative Sedierung gefordert. Sechs Leitlinien empfehlen, die Entscheidungen bezüglich künstlicher Nahrungs- und Flüssigkeitszufuhr unabhängig von der Entscheidung für oder gegen die Palliative Sedierung zu treffen (Schildmann und Schildmann 2014). Die Übersichtsarbeit offenbart aber auch kulturelle Unterschiede. Die japanische Leitlinie fordert beispielsweise zusätzlich zum Einverständnis der betroffenen PatientInnen auch ein Einverständnis von Familienangehörigen (Schildmann und Schildmann 2014).

\subsubsection{Medizinethische Kontroversen bezüglich der Palliativen Sedierung}

Die Palliative Sedierung wird sowohl von medizinischem Personal als auch von MedizinethikerInnen weitgehend als sinnvolle Maßnahme zur Linderung extremen Leids anerkannt (vgl. Jaspers et al. 2012; Müller-Busch 2004; Simon et al. 2007). Trotzdem ist das Thema im medizinethischen Diskurs weiterhin sehr präsent. Die Legitimität, die Ziele und die Grenzen der Palliativen Sedierung werden in der Literatur noch immer kontrovers diskutiert und auch bezüglich der Umsetzung in der klinischen Praxis scheinen Unsicherheiten und Divergenzen zu bestehen. Deshalb sollen im Folgenden einige dieser medizinethischen Kontroversen näher beleuchtet werden. 
Eine bedeutende medizinethische Kontroverse, die nicht nur im Kontext der Palliativen Sedierung eine Rolle spielt, betrifft einen möglichen Konflikt zwischen ärztlicher Indikation und PatientInnenwille. Wie bereits angesprochen, können PatientInnen nicht jegliche medizinische Maßnahme frei wählen, da stets eine von ÄrztInnen gestellte Indikation gegeben sein muss. Wenn Betroffene eine Palliative Sedierung wünschen, weil sie ihr Leiden als unerträglich empfinden, die behandelnden ÄrztInnen jedoch keine Indikation dafür sehen, ist eine Entscheidungsfindung schwierig. Es stellt ein bedeutendes Problem der klinischen Praxis dar, subjektives Leiden als Außenstehende beurteilen zu müssen und zu entscheiden, wann Belastungen stark genug sind, um eine Palliative Sedierung zu rechtfertigen (Bozzaro 2015; Kamprad und Helm 2018).

In Konfliktfällen wird von ÄrztInnen ein sorgfältiges Abwägen der Situation verlangt, da meist weder ein unreflektiertes Nachgeben, noch eine komplette Abweisung des PatientInnenwunsches im klinischen Alltag praktikabel sind. Alt-Epping und Nauck (2012) erörtern verschiedene Handlungsoptionen, um einer solchen Konfliktsituation zu begegnen. Zuweilen sei es möglich, PatientInnen an ärztliche KollegInnen zu verweisen, wenn die verantwortlichen ÄrztInnen die gewünschte Behandlung nicht durchführen wollen. Für viele Betroffene sei dies aber aufgrund begrenzter Mobilität und Lebenszeit nicht realisierbar.

Als eine weitere Handlungsoption wird die Erweiterung des klassischen Zwei-SäulenModells aus Indikation und Patientenwille angeführt. Wenn weitere Aspekte, wie die zu erwartenden Folgen einer Behandlung, die Wahrnehmung und die Nutzenbewertung der PatientInnen oder auch die Kosten einbezogen werden, könne eine für beide Seiten akzeptable Entscheidungsfindung erleichtert werden. Somit könne beispielsweise eine von PatientInnen gewünschte Maßnahme durchgeführt werden, wenn diese im Einklang mit dem Therapieziel steht, wenn sie in ihrer Wirksamkeit von den Betroffenen realistisch wahrgenommen wird und wenn deren Nutzen die Nachteile überwiegt (Alt-Epping und Nauck 2012).

Überdies wird ein Ansatz beschrieben, der die PatientInnenautonomie nicht mehr primär als reines Abwehrrecht definiert, sondern eine aktivere Einbindung der PatientInnen in den Entscheidungsprozess (shared decision making) umfasst. Dabei findet eine intensivere Interaktion zwischen PatientInnen und ÄrztInnen statt, die auch den Austausch über Moralvorstellungen beinhaltet und eine vertrauensvolle Beziehung zum Ziel hat. Schließlich bestehe eine weitere Möglichkeit der Konfliktlösung darin, den PatientInnenwunsch 
explizit in die Indikationsstellung einzubeziehen, um dem subjektiv empfundenen Leidensdruck der PatientInnen, der von außen nur schwer beurteilt werden kann, Rechnung zu tragen (Alt-Epping und Nauck 2012).

Eine weitere medizinethische Kontroverse betrifft den Einsatz der Palliativen Sedierung bei psychischen Symptomen. Sowohl die Rahmenleitlinie der EAPC als auch die Empfehlungen der AG Ethik am Lebensende in der AEM erkennen psychische Symptome grundsätzlich als Indikation für eine Palliative Sedierung an. Überdies merkt Simon (2008) an, dass Belastungen zumeist nicht klar in körperliches und psychisches Leid aufgeteilt werden können. Dennoch wird die Indikationsstellung bei psychischen Symptomen weithin als komplizierter eingeschätzt, weil sich die Refraktärität nur schwer nachweisen lässt, weil sich die Krankheitsverläufe oft dynamisch gestalten und weil schwere psychische Leiden nicht zwangsläufig nur in der Terminalphase einer unheilbaren Krankheit auftreten (Neitzke 2010; Schildmann und Schildmann 2014; Simon et al. 2007). Deshalb wird vielfach die Bedeutung einer sorgfältigen interdisziplinären Bewertung jedes Einzelfalls hervorgehoben (Oechsle et al. 2017). Die EAPC empfiehlt zudem, die Palliative Sedierung bei psychischen Symptomen nur in fortgeschrittenen Phasen schwerer Erkrankungen einzusetzen (Cherny et al. 2009).

Diese Empfehlung deutet bereits auf einen weiteren Konfliktpunkt hin, denn es besteht im medizinethischen Diskurs Unklarheit darüber, zu welchem Zeitpunkt einer Erkrankung die Palliative Sedierung eingesetzt werden darf. Es scheint in der medizinischen und medizinethischen Literatur größtenteils Konsens darüber zu bestehen, dass die Therapierefraktärität der Symptome als Voraussetzung für die Anwendung der Palliativen Sedierung gilt (Cherny et al. 2009; Neitzke et al. 2010; Oechsle et al. 2017).

In der Rahmenleitlinie der EAPC heißt es zudem, dass eine tiefe und kontinuierliche Sedierung nur in den letzten Lebenstagen und -stunden angewendet werden sollte (Cherny et al. 2009). Diese in der medizinethischen Literatur häufig vorgebrachte Meinung wird von Cellarius (2008) als imminence condition bezeichnet. Den Ursprung der imminence condition sieht der Autor in der Befürchtung, dass das Leben von PatientInnen, die möglicherweise noch Monate bis Jahre zu leben hätten, gezielt durch Palliative Sedierung verkürzt werden könnte. Auch wenn diese Argumentation nachvollziehbar ist, stellt sich die Frage, wie mit unerträglichem, therapierefraktärem Leiden umgegangen werden soll, wenn sich die Betroffenen noch nicht in der unmittelbaren Sterbephase befinden. Zudem ist der Eintritt in die Terminalphase nicht immer klar abgrenzbar (Cellarius 2008). 
Besonders kontrovers wird in der medizinethischen Literatur ein Szenario diskutiert, in dem PatientInnen unter einer unheilbaren und zum Tode führenden Krankheit leiden, sich jedoch noch nicht in der Terminalphase der Erkrankungen befinden. Eine solche Situation ist beispielsweise im Rahmen einer ALS denkbar. Können diese PatientInnen glaubhaft angeben, dass sie aufgrund des erwartbaren Krankheitsverlaufs unerträgliche psychische Symptome und existenzielles Leid erleben und deshalb eine Palliative Sedierung wünschen, stehen Behandler vor schwierigen Einzelfallentscheidungen (Müller-Busch 2008; Neitzke und Frewer 2004; Simon 2008).

Obwohl bereits vielfach eine Abgrenzung vorgenommen wurde, ist das Verhältnis der Palliativer Sedierung zur Sterbehilfe weiterhin Thema des medizinethischen Diskurses. Lipuma (2013) argumentiert, dass eine tiefe kontinuierliche Sedierung bis zum Eintreten des Todes mit einer Tötung auf Verlangen gleichzusetzen sei, da in beiden Fällen das Bewusstsein bis zum Eintreten des biologischen Todes irreversibel ausgeschaltet werde. Auch in der deutschen Übersetzung der EAPC-Rahmenleitlinie wird darauf hingewiesen, dass die Palliative Sedierung missbräuchlich angewendet werden könne, um das Leben der PatientInnen gezielt zu verkürzen:

„In der klinischen Praxis verabreichen manche Ärzte Medikation in sehr hohen Dosierungen, auf den ersten Blick, um Symptome zu lindern, jedoch mit einer impliziten Absicht der Lebenszeitverkürzung. Dies muss konstatiert werden bei einer freizügigen Sedierung von Patienten, die nicht unter refraktären Symptomen leiden, oder bei Dosierungen jenseits dessen, was zu einer adäquaten Beschwerdefreiheit nötig gewesen wäre“ (Alt-Epping et al. 2010).

Insbesondere in den Niederlanden und in Belgien wird eine Form der tiefen kontinuierlichen Sedierung bis zum Eintreten des Todes eingesetzt, die die Grenzen zur Tötung auf Verlangen verschwimmen lässt, da die betroffenen PatientInnen oft innerhalb von Stunden bis Tagen nach Beginn der Sedierung versterben (Chambaere et al. 2010; Rietjens et al. 2008). Es sei jedoch darauf hingewiesen, dass diese Form der Sedierung nicht der Definition der Palliativen Sedierung durch die EAPC entspricht. Erhebungen von MullerBusch et al. (2003), Müller-Busch et al. (2004) und Sitte et al. (2016) legen zudem nicht nahe, dass in Deutschland verbreitet eine Tötung auf Verlangen unter dem Deckmantel der Palliative Sedierung stattfindet. Beispielsweise zeigte die Befragung von MüllerBusch et al. (2004), dass die deutliche Mehrzahl der befragten ÄrztInnen (Mitglieder der Deutschen Gesellschaft für Palliativmedizin) die Legalisierung sowohl einer Tötung auf 
Verlangen als auch eines ärztlich assistierten Suizids ablehnten, während die Terminale Sedierung mehrheitlich positiv bewertet wurde.

Eine Palliative Sedierung im Sinne der EAPC-Definition - welche auch in dieser Arbeit zugrunde gelegt wird - hat nie das Ziel der absichtlichen Lebensverkürzung. Aus medizinethischer Perspektive unterscheidet sich dementsprechend die Palliative Sedierung hinsichtlich der Ziele (Symptomlinderung), der Durchführung (sedierende Medikation und Monitoring der Sedierungstiefe) und der Folgen (Bewusstseinsdämpfung) deutlich von einer Tötung auf Verlangen (Materstvedt et al. 2003; Weber et al. 2005).

Jedoch wird eine Lebensverkürzung in Kauf genommen, wenn diese als Nebenwirkung einer Sedierung auftritt, die notwendig ist, um anderweitig therapierefraktäre Symptome zu lindern. Eine solche Lebenszeitverkürzung wird zumeist mit Verweis auf das Konzept der Doppelwirkung akzeptiert (Cellarius 2008). Unter Voraussetzung eines sachgemäßen Einsatzes der Palliativen Sedierung, der ein adäquates Monitoring und eine entsprechende Medikamentendosierung einschließt, ist dieses Prinzip weithin anerkannt, auch weil die Alternative - die Inkaufnahme eines subjektiv unerträglichen Leidenszustands - ethisch fragwürdig erscheint (Beck 2004; Cellarius 2008; Morita et al. 2001).

Im Kontext der Diskussion um das Verhältnis von Palliativer Sedierung und Tötung auf Verlangen, spielt insbesondere die Frage nach Aufrechterhaltung lebenserhaltender Maßnahmen und begleitendem Monitoring eine Rolle. Wenn eine Palliative Sedierung bei gleichzeitigem Verzicht auf lebenserhaltende Maßnahmen gewünscht wird, ist davon auszugehen, dass die betroffenen PatientInnen innerhalb kurzer Zeit versterben. Dieses Szenario wird dementsprechend im medizinethischen Diskurs besonders kontrovers diskutiert (vgl. Müller-Busch et al. 2004; Neitzke und Frewer 2004; Simon et al. 2007).

Orentlicher (1997) vertritt die Meinung, dass eine Sedierung bei gleichzeitiger Beendigung lebenserhaltender Maßnahme mit Euthanasie gleichzusetzen sei. Das Prinzip der Doppelwirkung sei nicht anwendbar, da dadurch nur die Sedierung, nicht aber der Abbruch der lebenserhaltenden Maßnahmen zu rechtfertigen sei. Ein Verzicht auf lebenserhaltende Maßnahmen sei außerdem nicht mit Hinweis auf Leidenslinderung zu begründen, sondern verfolge ausschließlich das Ziel, das Leben zu verkürzen (Orentlicher 1997). Simon (2008) argumentiert hingegen, dass jeder Mensch ein Recht auf Ablehnung lebenserhaltender Maßnahmen habe und eine gleichzeitig vorliegende Indikation zur Palliativen Sedierung nicht durch diese Ablehnung aufgehoben werde. Die AG Ethik am Lebensende in der AEM kommt zu dem Schluss, dass im Falle einer reversibel angelegten 
Sedierung der Stufe I sämtliche Begleitmaßnahmen fortgesetzt oder gegebenenfalls neu begonnen werden sollten. Bei einer Sedierung der Stufe II sei die Lebenserhaltung hingegen nicht mehr vordergründig und einziges Therapieziel sei die Leidenslinderung, weshalb auf Begleitmaßnahmen, die den Sterbeprozess verlängern, verzichtet werden sollte (Neitzke et al. 2010).

Die geschilderten medizinethischen Kontroversen verdeutlichen, dass einige Aspekte der Palliativen Sedierung noch immer uneinheitlich bewertet werden. Auch für die Umsetzung in die klinische Praxis ergeben sich deshalb Unsicherheiten. Die Veröffentlichungen der EAPC und der AG Ethik am Lebensende in der AEM und die SOP „Palliative Sedierung“ bieten zwar eine erste Orientierung, doch können sie die beschriebenen Konflikte nicht vollends lösen. Um ÄrztInnen in Zukunft größere Handlungssicherheit zu ermöglichen, wird es notwendig sein, die bestehenden Rahmenpläne in Form von nationalen, lokalen oder klinikinternen Standards zu konkretisieren.

\subsubsection{Palliative Sedierung bei PatientInnen mit amyotropher Lateralsklerose}

In den letzten Jahren hat sich zunehmend ein Bewusstsein dafür entwickelt, dass auch nicht-onkologische PatientInnen von einer palliativmedizinischen Betreuung profitieren und dass palliativmedizinische Maßnahmen grundsätzlichen allen PatientInnen mit entsprechendem Bedarf zur Verfügung stehen sollten (Golla et al. 2007; Connolly et al. 2015). Im Zuge dieser Entwicklung sind allmählich auch neurologische Erkrankungen beispielsweise die multiple Sklerose, die ALS oder der Morbus Parkinson - in den erweiterten Fokus palliativmedizinischer Bemühungen gerückt. Weiterhin werden jedoch vor allem KrebspatientInnen in spezialisierten palliativmedizinischen Versorgungsstrukturen betreut (Bausewein 2005; Radbruch et al. 2003).

Bei der palliativmedizinischen Versorgung von PatientInnen mit neurologischen Erkrankungen ergeben sich einige besondere Herausforderungen, wie beispielsweise eine Einschränkung oder der Verlust der Kommunikationsfähigkeit. Dies kann eine Feststellung des PatientInnenwillens erschweren und eine große Belastung für Betroffene, Angehörige und Behandelnde darstellen. Auch Kognitionsstörungen, Lähmungen und Muskelkrämpfe treten im Rahmen neurologischer Erkrankungen gehäuft auf und müssen bei der palliativen Therapie berücksichtigt werden (Golla et al. 2007).

Im Folgenden soll die ALS näher beleuchtet werden, die als schwerwiegende neurologische Erkrankung auch palliativmedizinische Behandlungsprinzipien erfordert. Neben 
grundlegenden Informationen zum Krankheitsbild, werden besondere medizinethische Herausforderungen angesprochen, die sich im Kontext der palliativen Betreuung von ALS-PatientInnen ergeben.

\subsubsection{Grundlagen zur amyotrophen Lateralsklerose}

Die ALS ist eine Motoneuronenerkrankung und zeichnet sich durch eine degenerative Schädigung des ersten und zweiten Motoneurons aus. Charakteristische Symptome sind insbesondere der Verlust der Willkürmotorik, eine zentral aufsteigende und zuletzt die gesamte quergestreifte Muskulatur betreffende Lähmung und eine dadurch bedingte Dysphagie, Dysarthrie und respiratorische Insuffizienz. Auch treten Faszikulationen, Muskelkrämpfe und Gewichtsverlust auf. Ferner können gelegentlich eine erhöhte Affektdurchlässigkeit und Verhaltensstörungen beobachtet werden. Das Bewusstsein der PatientInnen bleibt in den meisten Fällen unbeeinträchtigt, jedoch kann in etwa $5 \%$ der ALSErkrankungen eine klinisch manifeste frontale Demenz diagnostiziert werden (Ludolph 2011; Ludolph und Anneser 2012).

Die Prävalenz der ALS liegt in Europa bei 6-8 pro 100000 Einwohnern, die Inzidenz bei 1,5-2 pro 100000 Einwohner und pro Jahr. Die Erkrankung wird überwiegend zwischen dem 40. und 70. Lebensjahr diagnostiziert und die mittlere Überlebenszeit nach Diagnose beträgt zwei bis fünf Jahre. Lebenszeitbegrenzend sind in der Regel eine respiratorische Insuffizienz und die daraus resultierende Hypoventilation. Die Betroffenen versterben zumeist in $\mathrm{CO}_{2}$-Narkose (Ludolph und Anneser 2012).

Bezüglich der Behandlung der ALS empfiehlt die Leitlinie der Deutschen Gesellschaft für Neurologie (DGN) einen multidisziplinären Ansatz, der sowohl eine Kausaltherapie als auch eine symptomatische Therapie einschließt. Dabei ist eine umfassenden Aufklärung der Betroffenen und gegebenenfalls der Angehörigen von großer Bedeutung, um gemeinsam Handlungsstrategien für die verschiedenen Krankheitsstadien und für mögliche Notfallsituationen festzulegen. Es kann sinnvoll sein, getroffene Entscheidungen in Form einer Patientenverfügung festzuhalten. Die Erkrankten sollten zudem frühzeitig darüber aufgeklärt werden, dass in der Regel kein Tod durch Ersticken zu erwarten ist, dass die Mehrheit der ALS-PatientInnen im Schlaf verstirbt und dass geeignete Medikamente zur Verfügung stehen, um Atemnot zu lindern (DGN 2015).

Eine Kausaltherapie der ALS besteht zurzeit vor allem in der Anwendung von Riluzol. Das antiglutamaterge Medikament hat neuroprotektive Wirkung und kann die Lebenszeit je nach Studie um einige Monate bis zu über einem Jahr verlängern (Ludolph und Anneser 
2012; DGN 2015). Auch kann seit einigen Jahren das Antioxidans Edaravone eingesetzt werden, welches seit 2015 in Japan und seit 2017 in den USA zugelassen ist. In Europa ist das Medikament hingegen noch nicht zugelassen und muss dementsprechend importiert werden (DGN 2017).

Die symptomatische Therapie hat das Ziel, die Lebensqualität der Betroffenen zu verbessern und die PatientInnenautonomie so weit wie möglich zu erhalten. Dabei spielen Maßnahmen wie Physio- und Ergotherapie, Logopädie, die Prophylaxe von Pneumonien und Thrombosen, die Therapie von Hypersalivation und Verschleimung der Atemwege und die psychosoziale Betreuung eine wichtige Rolle. Die Möglichkeit einer PEG-Anlage und einer nicht-invasiven Heimbeatmung sollte frühzeitig mit PatientInnen und Angehörigen erörtert werden (Connolly et al. 2015).

Auch eine invasive Beatmung mit Tracheotomie ist theoretisch möglich und hat das Potenzial, das Leben der Betroffenen deutlich zu verlängern. Jedoch schreitet die Muskellähmung unter der Beatmung weiter voran, was schließlich zu einem Locked-In-Syndrom führen kann. Die Mehrzahl der ALS-PatientInnen entscheidet sich nach Aufklärung gegen eine invasive Beatmung (Connolly et al. 2015; Kühnlein et al. 2008). Die konkreten Auswirkungen der verschiedenen Maßnahmen auf sowohl Lebensqualität als auch Lebenszeit sollten dementsprechend ausführlich mit den Betroffenen erörtert werden (Borasio und Voltz 2000; DGN 2015).

In einer von Tiirola et al. (2017) durchgeführten retrospektive Studie mit HospizpatientInnen wurde Atemnot als häufigstes Symptom in der Terminalphase der ALS angegeben, gefolgt von Schmerzen und Erschöpfung. Eine Behandlung der terminalen respiratorischen Insuffizienz ist zum Teil kausal möglich, wenn beispielsweise eine Pneumonie vorliegt, erfolgt jedoch vor allem symptomatisch durch die Gabe von Opioiden und gegebenenfalls Benzodiazepinen zur Anxiolyse. Die Schmerztherapie sollte laut Leitlinie entsprechend der WHO-Richtlinie initial durch nicht-sedierende, nichtsteroidale Antiphlogistika und im weiteren Verlauf durch Opioide erfolgen (DGN 2015). Die Einbindung von PalliativmedizinerInnen im fortgeschrittenen Krankheitsstadium wird in der Leitlinie zur ALS empfohlen, um eine möglichst gute Symptomkontrolle zu erzielen. Die Möglichkeit einer Palliativen Sedierung wird in der Leitlinie jedoch nicht explizit angesprochen (DGN 2015). 
1.1.5.2 Medizinethische Überlegungen zur Behandlung von ALS-PatientInnen Auf die große Bedeutung der Aufklärung und der Kommunikation mit den PatientInnen wurde im Verlauf dieser Arbeit bereits hingewiesen. Im Kontext einer rasch progredienten Krankheit wie der ALS, die in der Regel innerhalb weniger Jahre tödlich verläuft, präsentieren sich jedoch besondere Herausforderungen. Borasio und Voltz (2000) empfehlen, möglichst früh im Krankheitsprozess über den typischen Verlauf und über Behandlungsmöglichkeiten zu sprechen, um Unsicherheiten und Ängsten - insbesondere der Erstickungsangst - zu begegnen. Die Aufklärung über ein zu erwartendes Versterben im Schlaf könne die Betroffenen frühzeitig entlasten. Ein gezieltes Vorenthalten von Informationen sei ethisch nicht zu vertreten und zudem meist sinnlos, da ein Großteil der Betroffenen in der Lage sei, sich selbstständig über die Erkrankung zu informieren (Borasio und Voltz 2000).

Im Verlauf mehrerer Gespräche zwischen PatientInnen, medizinischem Personal und gegebenenfalls Angehörigen kann festgelegt werden, welche Maßnahmen in verschiedenen Krankheitsstadien ergriffen werden sollen. Es ist wichtig, darüber zu sprechen, inwieweit Lebensverlängerung als Behandlungsziel verfolgt werden soll. Lebensverlängernd wirken vor allem die Einnahme von Riluzol, künstliche Ernährung und Beatmung. Eine invasive Beatmung hat sogar das Potenzial, das Leben um einige Jahre zu verlängern, jedoch sollten die Betroffenen ausführlich über das Leben in einer Locked-In-Situation aufgeklärt werden (Jox 2017).

Eine nicht-invasive Beatmung hat primär das Ziel, die Lebensqualität zu verbessern, kann aber auch lebensverlängernd wirken (Kühnlein et al. 2008). Eine Ablehnung der invasiven Beatmung sollte möglichst schriftlich festgehalten werden, um die Intubation in einer Notfallsituation zu vermeiden. Weiterhin sollte verdeutlicht werden, dass eine Beatmung jederzeit beendet werden kann und dass in diesem Falle Medikamente zur Linderung der Atemnot eingesetzt werden können (Borasio und Voltz 2000).

Eine Beendigung der invasiven oder nicht-invasiven Beatmung muss erfolgen, wenn diese explizit von PatientInnen oder gerichtlich Bevollmächtigten gewünscht wird. Die Beendigung der Beatmung muss weiterhin von einer atemnotlindernden, gegebenenfalls sedierenden Medikation begleitet werden (Meyer et al. 2008). Obwohl die Fortsetzung einer Beatmung gegen den ausdrücklichen Willen der PatientInnen den Tatbestand der Körperverletzung erfüllt, scheinen viele ÄrztInnen zurückhaltend oder gar ablehnend auf 
den Wunsch nach elektiver Beatmungsbeendigung zu reagieren. Im Rahmen einer Befragung von Weber et al. (2001) gaben fast $50 \%$ der befragten ÄrztInnen an, dass das Abstellen einer künstlichen Beatmung eine Form der aktiven Sterbehilfe darstelle. In einer Studie von van Oorschot et al. (2005) setzten knapp $40 \%$ der befragten ÄrztInnen die Beendigung künstlicher Beatmung mit aktiver Sterbehilfe gleich. Es sei jedoch darauf hingewiesen, dass beide Studien schon etwas älter sind und dass sie nicht spezifisch die Beatmungsbeendigung bei ALS-PatientInnen thematisiert haben. Es scheint jedoch noch immer eine Unsicherheit bezüglich der aktuellen Rechtsprechung zu existieren, welche eine elektive Termination der Beatmung unter begleitender Medikation - beispielsweise mit Benzodiazepinen und Opioiden - ausdrücklich gestattet (Meyer et al. 2008).

Eine weitere medizinethische Herausforderung für die behandelnden ÄrztInnen kann entstehen, wenn sie von PatientInnen mit dem Wunsch nach Lebenszeitverkürzung konfrontiert werden. Jox (2017) argumentiert, dass es Betroffenen unabhängig von den Einstellungen der ÄrztInnen ermöglicht werden sollte, ihre Ängste und Wünsche auszudrücken und dass diese stets ernst genommen werden müssen. Das medizinische Personal sollte in offenen und vertrauensvollen Gesprächen versuchen, Empathie zu zeigen und die Motive der Betroffenen nachzuvollziehen. Auch sei es in solchen Situationen sinnvoll, wiederholt über die Krankheit, die Terminalphase und die Behandlungsmöglichkeiten aufzuklären und gegebenenfalls PalliativmedizinerInnen hinzuzuziehen (Jox 2017).

Um Ängsten zu begegnen, sollte den PatientInnen versichert werden, dass sie keinen Erstickungstod zu befürchten haben und dass geeignete Medikamente zur Verfügung stehen, um Atemnot zu bekämpfen (Meyer et al. 2008). Dies erscheint umso wichtiger vor dem Hintergrund einer prospektiven Studie, die von Maessen et al. (2014) in den Niederlanden durchgeführt wurde und in der über $40 \%$ der befragten ALS-PatientInnen angaben, Angst vor einem Versterben durch Ersticken zu haben.

Die Einbindung der Palliativmedizin in die Behandlung von ALS-PatientInnen wird von zahlreichen Autoren und ebenso in der Leitlinie zur ALS empfohlen (vgl. Andersen et al. 2012; Bausewein 2005; Borasio und Voltz 2000; DGN 2015; Jox 2017). Der Einsatz der Palliativen Sedierung ist aufgrund des typischen Verlaufs der ALS denkbar, wenn konventionelle Behandlungsmethoden keine ausreichende Symptomreduktion erreichen konnten (Müller-Busch 2008; Simon 2008). Eine Sedierung hat das Potenzial, sowohl die regelhaft auftretende Dyspnoe als auch die damit einhergehenden Ängste zu lindern: 
„Bei unkontrollierten Leidenssymptomen, die durch spezifische Medikamente nicht behandelbar sind, sollte die Möglichkeit einer palliativen Sedierung in Betracht gezogen werden, welche oft auch schon einen ausreichenden Effekt hat, wenn sie intermittierend für einige Tage durchgeführt wird. [...] Oft werden schon niedrige Dosen von Benzodiazepinen (ggf. mit sedierenden Neuroleptika) ausreichen, um eine Distanzierung von Ängsten und Leiden zu erreichen“ (Jox 2017).

\subsubsection{Zusammenfassung des aktuellen Forschungsstandes}

Die Palliative Sedierung verfolgt das Ziel der Symptomlinderung durch Bewusstseinsdämpfung in anderweitig therapierefraktären Situationen. Grundsätzlich kommen sowohl körperliche als auch psychische Symptome als Indikationen infrage. Ebenso wie für jede andere medizinische Maßnahme gelten ärztliche Indikation und Einwilligung der Betroffenen als Voraussetzung für die Durchführung einer Palliativen Sedierung. In der medizinischen und medizinethischen Literatur wird zudem die Therapierefraktärität der Symptome meist als Bedingung für die Indikationsstellung zur Palliativen Sedierung angesehen (Cherny et al. 2009; Neitzke et al. 2010).

Die Palliative Sedierung im Sinne der hier zugrunde liegenden Definition der EAPC verfolgt nicht das Ziel der Lebensverkürzung und ist deshalb von einer Tötung auf Verlangen abzugrenzen. Eine ungewollte Verkürzung des Lebens als Nebenwirkung sedierender Medikamente wird zumeist akzeptiert, wenn unerträgliche Belastungen nicht auf andere Weise gelindert werden können (Beck 2004; Nationaler Ethikrat 2006; Simon 2006).

Als medizinethische Grundlage der Palliativen Sedierung wurde die prinzipienorientierte Medizinethik nach Beauchamp und Childress (2001) beschrieben, die vier gleichwertige Prinzipien als Grundlage medizinischen Handelns beschreibt: Autonomie, Nichtschaden, Wohltun, Gerechtigkeit.

Die Empfehlungen der AG Ethik am Lebensende in der AEM (2010), die Rahmenleitlinie der EAPC (2009) und die SOP des CCC-Netzwerks (2017/2019) können im deutschen Kontext als Orientierung für den Einsatz der Palliativen Sedierung dienen. Auch wird die Palliative Sedierung in der aktuellen S3-Leitlinie Palliativmedizin als mögliche Maßnahme zur Linderung therapierefraktärer Symptome am Lebensende (bei onkologischen PatientInnen) genannt.

Medizinethische Kontroversen bezüglich der Palliativen Sedierung betreffen insbesondere einen möglichen Konflikt zwischen ärztlicher Indikation und PatientInnenwunsch, 
die Indikationsstellung bei psychischer Symptomatik, die Beschränkung der Palliativen Sedierung auf die unmittelbare Sterbephase und den gleichzeitigen Verzicht auf lebenserhaltende Maßnahmen (Bozzaro 2015; Cellarius 2008; Cherny et al. 2009; Kamprad und Helm 2018; Neitzke et al. 2010; Simon 2008)

In der vorliegenden Arbeit wird die Palliative Sedierung im Kontext der ALS thematisiert. Aufgrund der progredienten Symptomatik der ALS wird eine palliativmedizinische Mitbetreuung unter anderem in der Leitlinie zur ALS empfohlen (DGN 2015). Auch der Einsatz einer Palliativen Sedierung erscheint aufgrund des typischen Verlaufs der Erkrankung denkbar (Jox 2017; Simon 2008). 


\subsection{Forschungsfragen}

Der vorangegangene Einblick in den medizinethischen Diskurs zeigt, dass die Palliative Sedierung weitestgehend als Möglichkeit zur Linderung stark belastender und anderweitig therapierefraktärer Symptome akzeptiert ist. Sie wird in der medizinethischen Literatur häufig im Kontext der Behandlung von KrebspatientInnen erörtert (AWMF 2018; Bausewein 2005; Beck 2004; Jaspers et al. 2012).

Seltener wird die Palliative Sedierung im Kontext neurologischer Erkrankungen - wie beispielsweise der ALS - thematisiert (vgl. Jox 2017; Müller-Busch 2008; Simon 2008). Aufgrund der nur vereinzelten Hinweise auf eine Palliativen Sedierung bei ALS-PatientInnen, ist unklar, ob und in welchem Ausmaß diese im klinischen Alltag zum Einsatz kommt. Jedoch scheint es durchaus denkbar, dass Sedierungspraktiken existieren, die einerseits nicht standardisiert sind und andererseits nicht immer offiziell den Titel der Palliativen Sedierung tragen. Aus diesen Überlegungen ergibt sich die folgende erste Forschungsfrage:

1. Kommt die Palliative Sedierung bei der Behandlung von ALS-PatientInnen zum Einsatz?

Des Weiteren haben sich im medizinethischen Diskurs einige Streitpunkte bezüglich der Palliativen Sedierung herauskristallisiert. Der Einsatz einer flachen oder intermittierenden Sedierung wird zumeist nicht als kritisch beurteilt, da eine solche Maßnahme grundsätzlich reversibel angelegt ist. Strittig ist vielmehr die Anwendung einer tiefen und kontinuierlichen Sedierung, insbesondere wenn diese mit dem Verzicht auf Monitoring, künstliche Nahrungs- oder Flüssigkeitszufuhr verbunden ist. Auch die kontroverse Frage nach dem Einsatz der Palliativen Sedierung bei psychischen Symptomen spielt im Rahmen der ALS-Behandlung eine Rolle, da der zu erwartende Krankheitsverlauf zu Depressionen, Angstzuständen und existenziellem Leid auf Seiten der Betroffenen führen kann (DGN 2015).

Es stellt sich jedoch die Frage, inwieweit sich diese theoretischen medizinethischen Kontroversen im klinischen Alltag wiederfinden. Im empirischen Teil dieser Arbeit soll dementsprechend untersucht werden, ob diese strittigen Aspekte das Entscheidungsverhalten von ÄrztInnen in der klinischen Praxis beeinflussen. Aus diesen Überlegungen ergeben sich die folgenden zwei Forschungsfragen: 
2. Inwiefern würden behandelnde ÄrztInnen dem Wunsch von ALS-PatientInnen nach kontinuierlicher und tiefer Sedierung zustimmen, um einerseits körperliche und andererseits psychische Symptome zu lindern?

3. Inwiefern beeinflusst der gleichzeitige Wunsch nach Verzicht auf künstliche Ernährung die Bewertung der Palliativen Sedierung?

Im Rahmen dieser Fragen soll zunächst ermittelt werden, ob behandelnde ÄrztInnen überhaupt einer tiefen und kontinuierlichen Sedierung zustimmen würden und ob eine $\mathrm{Zu}$ stimmung eher bei körperlichen als bei psychischen Symptomen erfolgt. Auch die persönliche Einstellung bezüglich des angemessenen Zeitpunkts für eine Palliative Sedierung soll dabei erfragt werden. In Frage 3 steht die Bewertung der Palliativen Sedierung bei gleichzeitigem Abbruch lebenserhaltender Maßnahmen im Vordergrund. In diesem Kontext soll zusätzlich eruiert werden, ob das Vorliegen einer Patientenverfügung Einfluss auf die Bewertung nimmt und inwiefern behandelnde ÄrztInnen eine Nähe dieses Vorgehens zur Tötung auf Verlangen empfinden.

Die nicht-invasive Beatmung bietet ALS-PatientInnen die Möglichkeit, ihre Lebensqualität über einige Monate hinweg aufrecht zu erhalten. Empfinden die PatientInnen ihre Lebensqualität unter der Beatmung als nicht mehr erträglich, haben sie die Möglichkeit, diese abzubrechen (Meyer et al. 2008). Eine solche Entscheidung ist rechtlich eindeutig gestattet, da jede Person lebenserhaltende Maßnahmen ablehnen darf. Eine begleitende palliative, sedierende Medikation während und nach der Beatmungsbeendigung ist dringend geboten und ebenfalls rechtlich erlaubt. Dennoch gibt es in der Literatur Hinweise darauf, dass behandelnde ÄrztInnen eine solche elektive Beatmungsbeendigung bei begleitender palliativer Sedierung nicht selten kritisch sehen oder gar ablehnen (Meyer et al. 2008; van Oorschot et al. 2005; Weber et al. 2001).

Da eine Beendigung der invasiven oder nicht-invasiven Beatmung dringend von palliativen Maßnahmen begleitet werden sollte, haben ALS-PatientInnen ohne Unterstützung der behandelnden ÄrztInnen kaum eine Möglichkeit, ihr Recht auf Beatmungsbeendigung auszuüben. Weil eine Ablehnung der Beatmungsbeendigung auf Seiten der ÄrztInnen schwerwiegende Konsequenzen für die Betroffenen hat, soll folgende Frage beantwortet werden:

4. Wie beurteilen behandelnde ÄrztInnen den PatientInnenwunsch nach elektiver Beatmungsbeendigung bei gleichzeitiger Palliativer Sedierung? 
Auch im Kontext dieser Frage soll ermittelt werden, inwiefern das Vorliegen einer Patientenverfügung die Bewertung beeinflusst und ob behandelnde ÄrztInnen eine Nähe des Vorgehens zur Tötung auf Verlangen ausmachen.

Schließlich soll untersucht werden, ob die Einstellungen behandelnder ÄrztInnen zur Palliativen Sedierung bei ALS-PatientInnen durch bestimmte Kontextfaktoren beeinflusst werden. Beispielsweise ist zu vermuten, dass sich ÄrztInnen mit Zusatz-Weiterbildung Palliativmedizin bereits intensiver mit dem Thema der Palliativen Sedierung beschäftigt haben und diese positiver bewerten als ÄrztInnen ohne die Weiterbildung. Auch die persönliche Erfahrung mit der Behandlung von ALS-PatientInnen beeinflusst vermutlich die Einstellung der ÄrztInnen. Die letzte Forschungsfrage dient der Erfassung solcher Korrelationen:

5. Inwiefern wird das Antwortverhalten durch die Berufserfahrung und die Aus- und Weiterbildung der teilnehmenden ÄrztInnen beeinflusst? 


\section{Material und Methoden}

Um die im vorangegangenen Kapitel beschriebenen Forschungsfragen zu beantworten, wurde eine bundesweite Fragebogenstudie mit ÄrztInnen mit Weiterbildungsermächtigung in den Bereichen Neurologie oder Palliativmedizin durchgeführt. Die Ethikkommission der Universitätsmedizin Göttingen wurde im Vorfeld der Umfrage über Design und Forschungsanliegen informiert. Ein Antrag wurde von der Kommission als nicht erforderlich angesehen.

\subsection{Auswahl der Teilnehmenden}

Als Probanden für die Befragung wurden ÄrztInnen mit Weiterbildungsermächtigung im Bereich der Neurologie und/oder der Palliativmedizin ausgewählt. Diese Auswahl beruht auf der Annahme, dass die Behandlung von ALS-PatientInnen in erster Linie durch NeurologInnen erfolgt, während ÄrztInnen mit Zusatz-Weiterbildung Palliativmedizin vermutlich die meiste Erfahrung mit Palliativer Sedierung aufweisen. Da die Zusatz-Weiterbildung Palliativmedizin prinzipiell von FachärztInnen jedes Fachgebiets absolviert werden kann, ist jedoch eine Heterogenität der Facharztausbildungen innerhalb dieser Gruppe zu erwarten.

Die Beschränkung auf weiterbildungsermächtigte und damit erfahrene ÄrztInnen erschien sinnvoll, da diese mit größerer Wahrscheinlichkeit bereits in Kontakt mit ALSPatientInnen und Palliativer Sedierung gekommen sind und bei der Beantwortung des Fragebogens auf persönliche Erfahrungen zurückgreifen können. Es ist zudem davon auszugehen, dass Entscheidungen bezüglich Palliativer Sedierung im Endstadium einer ALS-Erkrankung in der Regel von erfahrenen FachärztInnen getroffen werden. Außerdem konnten weiterbildungsermächtigte ÄrztInnen problemlos über die Internetseiten der Ärztekammern kontaktiert werden.

Die Daten sämtlicher ÄrztInnen mit Weiterbildungsermächtigung in den Bereichen Neurologie und Palliativmedizin wurden den Internetseiten der Landes- und Bezirksärztekammern (Stand März 2018) entnommen. Auf diese Weise wurden zunächst Name, Arbeitsplatz, Adresse, Bundesland und Weiterbildungsermächtigung von 1794 Personen darunter 1149 ÄrztInnen mit Weiterbildungsermächtigung Neurologie und 645 ÄrztInnen mit Weiterbildungsermächtigung Palliativmedizin - erfasst. Zur Teilnahme an der Studie wurden daraus 570 ÄrztInnen mit Weiterbildungsermächtigung Neurologie und 423 ÄrztInnen mit Weiterbildungsermächtigung Palliativmedizin zufällig ausgewählt. Im 
Falle einer gemeinsamen Weiterbildungsermächtigung mehrerer ÄrztInnen, wurde jede Person einzeln aufgeführt und kontaktiert. Fehlende Angaben bezüglich der Adressen auf den Internetseiten der Ärztekammern wurden durch Internetrecherche ergänzt.

\subsection{Aufbau des Fragebogens}

Der Fragebogen (siehe Anhang) lässt sich grob in drei Abschnitte gliedern. Am Anfang steht eine kurze Definition der Palliativen Sedierung, um eine gemeinsame Wissensgrundlage für alle Teilnehmenden zu schaffen. Darauffolgend wird erfragt, inwiefern bereits eine Beschäftigung mit dem Thema der Palliativen Sedierung stattgefunden hat. Die Teilnehmenden sollen außerdem angeben, wie lange sie bereits ALS-PatientInnen behandeln, wie viele ALS-PatientInnen sie durchschnittlich pro Monat behandeln und ob sie bereits eine Palliative Sedierung bei ALS-PatientInnen durchgeführt haben. Sollte dies der Fall sein, wird in einem Freitext-Feld nach den Symptomen gefragt, die zur Entscheidung für eine Palliative Sedierung geführt haben.

Der Hauptteil des Fragebogens beinhaltet drei Fallbeispiele, die von den Teilnehmenden bezüglich des Einsatzes der Palliativen Sedierung bewertet werden sollen. Im ersten Szenario wird der Fall einer ALS-Patientin im fortgeschrittenen Krankheitsstadium beschrieben, die unter starken körperlichen Symptomen leidet, welche bisher nicht ausreichend gelindert werden konnten. Deshalb wünscht sie eine kontinuierliche und tiefe Sedierung. Folgende Aussagen (Items) zum Umgang mit diesem Wunsch sollten mithilfe einer vierstufigen Skala (,Stimme völlig zu“, „Stimme eher zu“, „Stimme eher nicht zu“, „Stimme nicht $\mathrm{zu}^{\circ}$ ) bewertet werden:

Tabelle 1: Items Szenario 1.1

Der Wunsch der Patientin ist für mich nachvollziehbar.

Die Patientin hat ein Recht darauf, dass ihrem Wunsch entsprochen wird.

Ich würde dem Wunsch nur nachkommen, wenn vorherige Versuche einer flachen bzw. intermittierenden Sedierung keine ausreichende Symptomlinderung erzielen konnten.

Ich würde dem Wunsch nur nachkommen, wenn sich die Patientin bereits unmittelbar in der Sterbephase befindet.

Ich würde generell keine kontinuierliche tiefe Sedierung durchführen. 
In einem nächsten Schritt wird zusätzlich angegeben, dass dieselbe Patientin einen gleichzeitigen Verzicht auf künstliche Ernährung wünscht. Die Teilnehmenden werden wiederum gebeten, ihre Zustimmung $\mathrm{zu}$ folgenden Aussagen auf einer vierstufigen Skala (,Stimme völlig zu“, „Stimme eher zu“, „Stimme eher nicht zu“, „Stimme nicht zu“) zu bewerten:

Tabelle 2: Items Szenario 1.2

Der Wunsch der Patientin ist für mich nachvollziehbar.

Die Patientin hat ein Recht darauf, dass ihrem Wunsch entsprochen wird.

Ich würde dem Wunsch nur nachkommen, wenn dieser in einer Patientenverfügung dokumentiert ist.

Ich würde dem Wunsch nur nachkommen, wenn sich die Patientin bereits unmittelbar in der Sterbephase befindet.

Der Verzicht auf künstliche Ernährung bei kontinuierlicher tiefer Sedierung wäre in meinen Augen mit einer Tötung auf Verlangen gleichzusetzen.

Der Verzicht auf künstliche Ernährung bei kontinuierlicher und tiefer Sedierung führt zu einem früheren Versterben als es dem Erkrankungsverlauf ohne diese Maßnahme entsprechen würde.

Eine künstliche Ernährung bei einer kontinuierlichen tiefen Sedierung wäre in meinen Augen medizinisch nicht indiziert.

Schließlich werden die Teilnehmenden gebeten anzugeben, ob sie es im Rahmen des geschilderten Szenarios als moralisch belastender empfinden würden, eine bestehende künstliche Ernährung mit Beginn der Sedierung zu beenden oder eine erst durch die Sedierung erforderlich werdende künstliche Ernährung nicht neu zu beginnen.

Im zweiten Fallbeispiel wird der Fall einer ALS-Patientin im fortgeschrittenen Krankheitsstadium beschrieben, die unter refraktärer psychischer und existenzieller Symptomatik leidet, welche bisher nicht ausreichend gelindert werden konnte. Die Patientin wünscht deshalb eine tiefe und kontinuierlich Sedierung. In einem zweiten Schritt wird wiederum der Wunsch nach Verzicht auf künstliche Ernährung hinzugefügt. Die zu bewertenden Aussagen und die vierstufige Bewertungsskala sind mit denen des ersten Szenarios identisch.

Im dritten Fallbeispiel wird ein ALS-Patient beschrieben, der aufgrund respiratorischer Insuffizienz seit einigen Monaten invasiv beatmet wird und nun aufgrund der starken körperlichen Symptomatik eine Beendigung der Beatmung wünscht. Die Teilnehmenden 
werden gebeten, folgende Aussagen mithilfe einer vierstufigen Zustimmungsskala (,Stimme völlig zu“, „Stimme eher zu“, „Stimme eher nicht zu“, „Stimme nicht zu“) zu bewerten:

Tabelle 3: Items Szenario 3

Der Wunsch des Patienten ist für mich nachvollziehbar.

Der Patient hat ein Recht darauf, dass seinem Wunsch entsprochen wird.

Ich würde dem Wunsch nur nachkommen, wenn dieser in einer Patientenverfügung dokumentiert ist.

Ich würde dem Wunsch nur nachkommen, wenn sich der Patient bereits unmittelbar in der Sterbephase befindet.

Die Beendigung der invasiven Beatmung unter Sedierung wäre in meinen Augen mit einer Tötung auf Verlangen gleichzusetzen.

Im letzten Teil des Fragebogens werden persönliche Daten der Teilnehmenden - Geschlecht, Alter, Facharztausbildungen und die Zusatz-Weiterbildung Palliativmedizin erfragt. Schließlich besteht die Möglichkeit, in einem Freitext-Feld Anmerkungen zum Thema der Befragung oder zur Befragung selbst zu äußern. 


\subsection{Erstellung des Fragebogens und Durchführung der Befragung}

Ausgehend von den geschilderten theoretischen Überlegungen, wurde der Fragebogen erstellt und im Rahmen eines Expertenratings überarbeitet. Dazu wurde der Fragebogen Mitgliedern des Instituts für Ethik und Geschichte der Medizin, der Klinik für Palliativmedizin und der Klinik für Neurologie der Universitätsmedizin Göttingen vorgelegt und hinsichtlich des Aufbaus, der Verständlichkeit, der Vollständigkeit und der praktischen Relevanz überprüft. Anhand der resultierenden Verbesserungsvorschläge wurde der Fragebogen überarbeitet. Zur Erstellung der Fragebögen und für die nachfolgende Erfassung und Verifikation der Fragebogendaten wurde das Programm EvaSys verwendet.

Im Mai 2018 wurden insgesamt 993 Fragebögen per Post versendet. Dem Fragebogen lagen jeweils ein Anschreiben und ein Deckblatt bei (siehe Anhang). Im Anschreiben wurden das Forschungsanliegen und das Vorgehen bei der anonymisierten Auswertung kurz erläutert. Auf dem Deckblatt wurden die TeilnehmerInnen gebeten, den ausgefüllten Fragebogen innerhalb von vier Wochen zurückzusenden. Weiterhin sollten Name und Adresse des Absenders auf dem Deckblatt vermerkt werden, um diese Personen vom Recall auszuschließen. Auch konnte auf dem Deckblatt angegeben werden, ob Interesse daran besteht, die Ergebnisse der Studie per Post zu erhalten. Außerdem lag ein adressierter, nicht frankierter Rückumschlag bei.

Nach Ablauf der ersten Rücksendefrist wurden zunächst die Deckblätter von den ausgefüllten Fragebögen abgetrennt, sodass keine Zuordnung von Adressen und Fragebögen mehr möglich war. Mithilfe der Deckblätter wurden diejenigen ÄrztInnen ermittelt, die bereits geantwortet hatten oder deren Briefe nicht zugestellt werden konnten. Einige Fragebögen wurden jedoch auch ohne Deckblatt oder ohne Absenderadresse zurückgeschickt, sodass nicht alle Adressen mit Hinblick auf den Recall erfasst werden konnten.

Im Juli 2018 wurden im Rahmen des Recalls 725 Personen erneut mit der Bitte kontaktiert, den Fragebogen auszufüllen. Es wurde zudem darauf hingewiesen, dass der Fragebogen gerne erneut übersendet werden könne. Weiterhin wurde ein kurzer Recall-Fragebogen (siehe Anhang) beigelegt, auf dem die Befragten angeben konnten, warum sie nicht an der Umfrage teilnehmen möchten. Der Fragebogen wurde auf Nachfrage noch einmal an 23 ÄrztInnen versendet. 


\subsection{Auswertung der Fragebögen}

Die Auswertung der Fragebögen erfolgte anonymisiert und unabhängig von den Informationen des Deckblatts. Die Deckblätter mit Absenderadressen wurden lediglich zur Durchführung des Recalls verwendet und anschließend vernichtet. Die Daten der Fragebögen wurden mithilfe des Programms EvaSys erfasst, verifiziert und formatiert. Die statistische Auswertung der Daten erfolgte mit der Statistik-Software Statistica 13.3 und SPSS 25.0.

Für alle statistischen Tests wurde ein globales Signifikanzniveau von $5 \%(\mathrm{p}<0,05)$ festgelegt und bei multiplen Tests mithilfe der Bonferroni-Korrektur adjustiert. Für den Vergleich der Tendenzen von zwei unabhängigen Stichproben, bei ordinalskalierten abhängigen Variablen, wurde der Mann-Whitney-U-Test verwendet. Sollten mehr als zwei unabhängige Stichproben verglichen werden, so wurde bei ordinalskalierten unabhängigen Variablen der Kruskal-Wallis-Test eingesetzt. Für den Vergleich zweier verbundener Stichproben, bei ordinalskalierten abhängigen Variablen, wurde der Wilcoxon-Test für gepaarte Stichproben genutzt. Nominalskalierte Variablen wurden mithilfe des Chi-Square-Tests ausgewertet. Die Freitextantworten und der kurze Recall-Fragebogen wurden separat und ohne Nutzung einer Statistik-Software ausgewertet. 


\section{Ergebnisse}

Insgesamt wurden 296 Fragebögen zurückgesendet und ausgewertet, was einem Rücklauf von etwa $30 \%$ entspricht. Jedoch wurde keine Frage von 296 Personen beantwortet, da einerseits im Rahmen der Fallbeispiele „Keine Angabe“ gewählt werden konnte und andererseits bei einigen Fragen weder eine Antwort noch „Keine Angabe“ angekreuzt wurde. Die folgenden im Text und in den Abbildungen genannten Prozentangaben beziehen sich dementsprechend auf die gültigen Antworten.

Zudem wurden 92 Recall-Fragebögen (siehe Anhang) zurückgeschickt, auf denen Gründe für die Nichtteilnahme angegeben werden konnten (Mehrfachnennungen möglich). Darunter befanden sich zehn Bögen mit dem Hinweis darauf, dass der Fragebogen bereits ausgefüllt wurde oder mit der Bitte um ein erneutes Zusenden des Fragebogens.

Zusätzlich gaben vier Personen per Mail an, dass sie nicht an der Befragung teilnehmen möchten, da sie keine ALS-PatientInnen (im Endstadium der Erkrankung) behandeln. Weitere fünf Personen gaben im Freitextfeld am Ende des Fragebogens („Haben Sie weitere Anmerkungen zum Thema oder zur Befragung?") Gründe für die Nichtteilnahme an. Darunter verwiesen vier Personen auf fehlende Erfahrung mit ALS-PatientInnen und eine Person gab zeitliche Gründe an (siehe Anhang).

Auf 39 der 92 ausgewerteten Recall-Fragebögen wurde die Antwortmöglichkeit „Mir ist die Beantwortung aus zeitlichen Gründen nicht möglich“" angekreuzt, 6 Personen gaben datenschutzrechtliche Bedenken und eine Person moralische Bedenken als Grund für die Nichtteilnahme an. Zudem hatten 42 Personen eine Freitextantwort (siehe Anhang) formuliert. In der Mehrzahl der Freitextantworten wurde fehlende Erfahrung mit ALS-PatientInnen oder mit Palliativer Sedierung als Grund für die Nichtteilnahme genannt.

Im Folgenden werden die Ergebnisse der Fragebogenstudie zunächst deskriptiv dargestellt. Zudem werden signifikante Unterschiede im Antwortverhalten zwischen den folgenden Gruppen hervorgehoben:

Tabelle 4: Analysierte unabhängige Variablen

Zusatz-Weiterbildung Palliativmedizin absolviert: Ja/Nein
Bereits Palliative Sedierung bei ALS-PatientInnen durchgeführt: Ja/Nein
$\begin{aligned} & \text { Bisherige Beschäftigung mit der Thematik: Intensive Beschäftigung/Wenig oder fehlende } \\ & \text { Beschäftigung }\end{aligned}$


Ebenso wurde das Antwortverhalten männlicher und weiblicher TeilnehmerInnen verglichen und in einigen Fällen auf Unterschiede zwischen den Altersgruppen getestet. Im Folgenden werden vor allem signifikante Unterschiede zwischen den genannten Gruppen beschrieben. Nicht signifikante Ergebnisse werden nur thematisiert, insofern sie im Kontext der Forschungsfragen relevant erscheinen.

Anschließend werden signifikante Unterschiede im Antwortverhalten zwischen den verschiedenen Fallbeispielen $(1.1,1.2,2.1,2.2,3)$ dargestellt. Auch in diesem Rahmen wird anschließend analysiert, ob die Zusatz-Weiterbildung Palliativmedizin, die Erfahrung mit Palliativer Sedierung von ALS-PatientInnen und die bisherige Beschäftigung mit der Thematik zu signifikanten Unterschieden im Antwortverhalten geführt haben. 


\subsection{Soziodemographische Daten}

Tabelle 5 fasst die soziodemographischen Daten der Studienteilnehmenden zusammen. Knapp $85 \%$ der Teilnehmenden waren zum Zeitpunkt der Befragung über 45 Jahre alt, was sich durch die gezielte Auswahl von weiterbildungsermächtigten ÄrztInnen erklären lässt. Die restlichen $15 \%$ waren zwischen 35 und 45 Jahre alt.

Außerdem haben mit etwa $30 \%$ aller Teilnehmenden deutlich weniger Frauen als Männer an der Befragung teilgenommen, jedoch entspricht diese Verteilung etwa der Geschlechterverteilung aller kontaktierten Personen (26 \% Frauen und 74 \% Männer).

Mit $44 \%$ wurde die Neurologie als häufigste Facharztausbildung genannt, gefolgt von der Inneren Medizin und der Anästhesiologie. Zudem gaben 63 \% der Teilnehmenden an, die Zusatz-Weiterbildung Palliativmedizin absolviert zu haben.

Tabelle 5: Soziodemographische Daten

\begin{tabular}{|l|l|}
\hline Alter & $\mathbf{N}=\mathbf{2 8 9}$ \\
Unter 35 Jahre & $\mathrm{N}=0(0 \%)$ \\
35-45 Jahre & $\mathrm{N}=44(15,2 \%)$ \\
45-55 Jahre & $\mathrm{N}=119(41,2 \%)$ \\
55-65 Jahre & $\mathrm{N}=115(39,8 \%)$ \\
Über 65 Jahre & $\mathrm{N}=11(3,8 \%)$ \\
\hline Geschlecht & $\mathbf{N}=\mathbf{2 9 1}$ \\
Weiblich & $\mathrm{N}=87(29,9 \%)$ \\
Männlich & $\mathrm{N}=203(69,8 \%)$ \\
Anderes/Keine Angabe & $\mathrm{N}=1(0,3 \%)$ \\
\hline Facharztausbildung & $\mathbf{N}=\mathbf{2 9 1}$ \\
Neurologie & $\mathrm{N}=129(44,3 \%)$ \\
Innere Medizin & $\mathrm{N}=78(26,8 \%)$ \\
Anästhesiologie & $\mathrm{N}=53(18,2 \%)$ \\
Sonstige & $\mathrm{N}=61(21 \%)$ \\
\hline Zusatz-Weiterbildung Palliativmedizin & $\mathbf{N}=\mathbf{2 9 1}$ \\
Ja & $\mathrm{N}=182(62,8 \%)$ \\
Nein & $\mathrm{N}=108(37,2 \%)$ \\
\hline
\end{tabular}




\subsection{Allgemeine Fragen zur Palliativen Sedierung und zur Behandlung von ALS-PatientInnen}

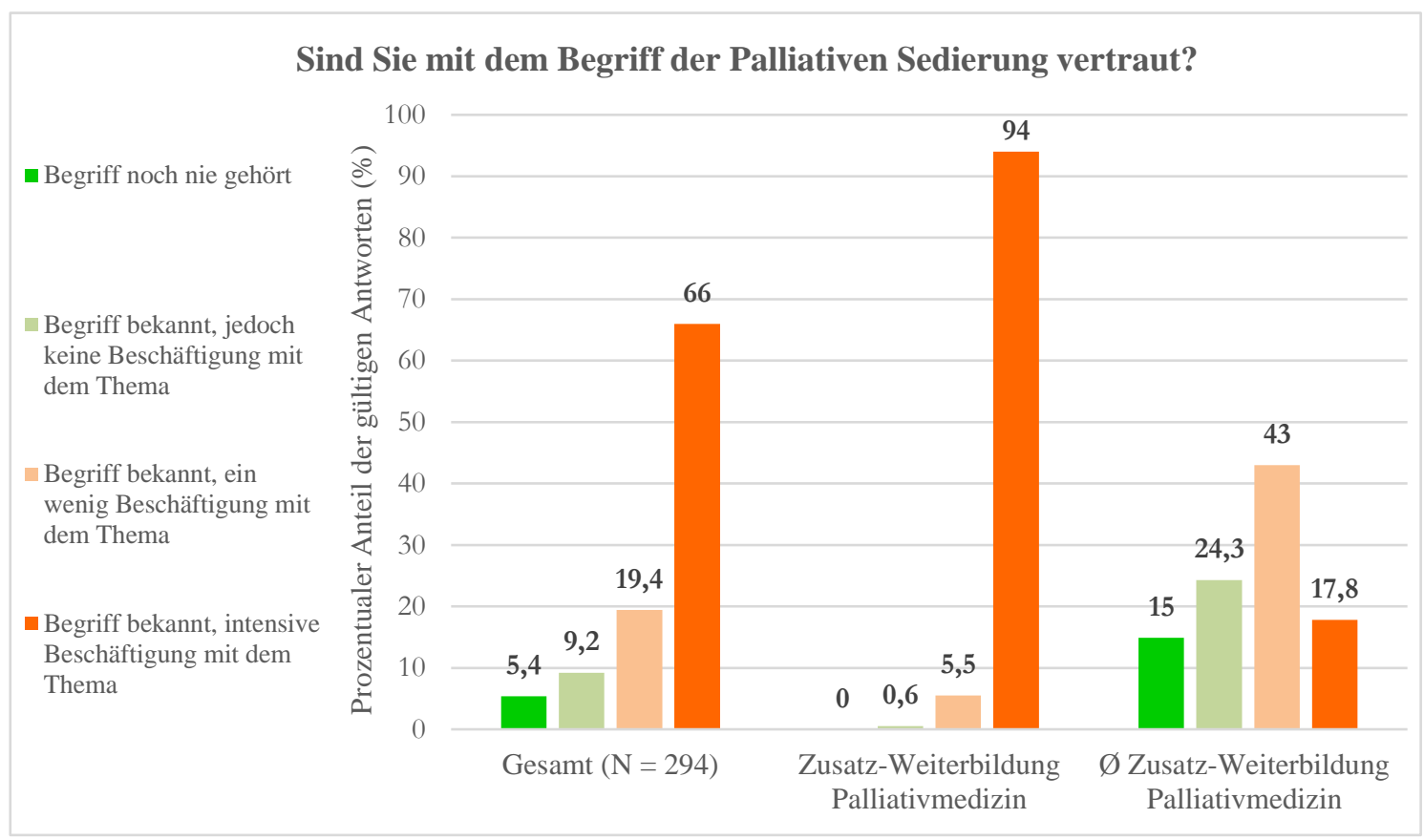

Abbildung 1: Übersicht Begriffskenntnis

Abbildung 1 zeigt die Selbsteinschätzung der Teilnehmenden bezüglich ihrer bisherigen Beschäftigung mit dem Begriff der Palliativen Sedierung. Insgesamt hatten $5 \%$ der Antwortenden den Begriff noch nie gehört und $66 \%$ hatten sich bereits intensiv mit der Thematik beschäftigt.

Es besteht ein statistisch signifikanter Unterschied ( $\mathrm{p}<0,00001)$ zwischen den teilnehmenden ÄrztInnen mit und ohne Zusatz-Weiterbildung Palliativmedizin. Während sich in der Gruppe mit Zusatz-Weiterbildung $94 \%$ bereits intensiv mit dem Begriff der Palliativen Sedierung beschäftigt hatten, trifft dies in der Gruppe ohne Zusatz-Weiterbildung nur auf $18 \%$ zu.

Es können hinsichtlich der vorangegangenen Beschäftigung mit der Palliativen Sedierung keine signifikanten Unterschiede zwischen den verschiedenen Altersgruppen und zwischen männlichem und weiblichem Geschlecht festgestellt werden. 
Weiterhin wurde erfragt, wie viele ALS-PatientInnen die Teilnehmenden im Durchschnitt pro Monat behandeln. Eine Übersicht der Ergebnisse ist in Abbildung 2 dargestellt. Insgesamt gaben $77 \%$ der teilnehmenden ÄrztInnen an, weniger als drei ALS-PatientInnen pro Monat zu behandeln. Etwa $17 \%$ der Antwortenden behandelten keine ALS-PatientInnen und nur knapp $6 \%$ behandelten über drei ALS-PatientInnen pro Monat.

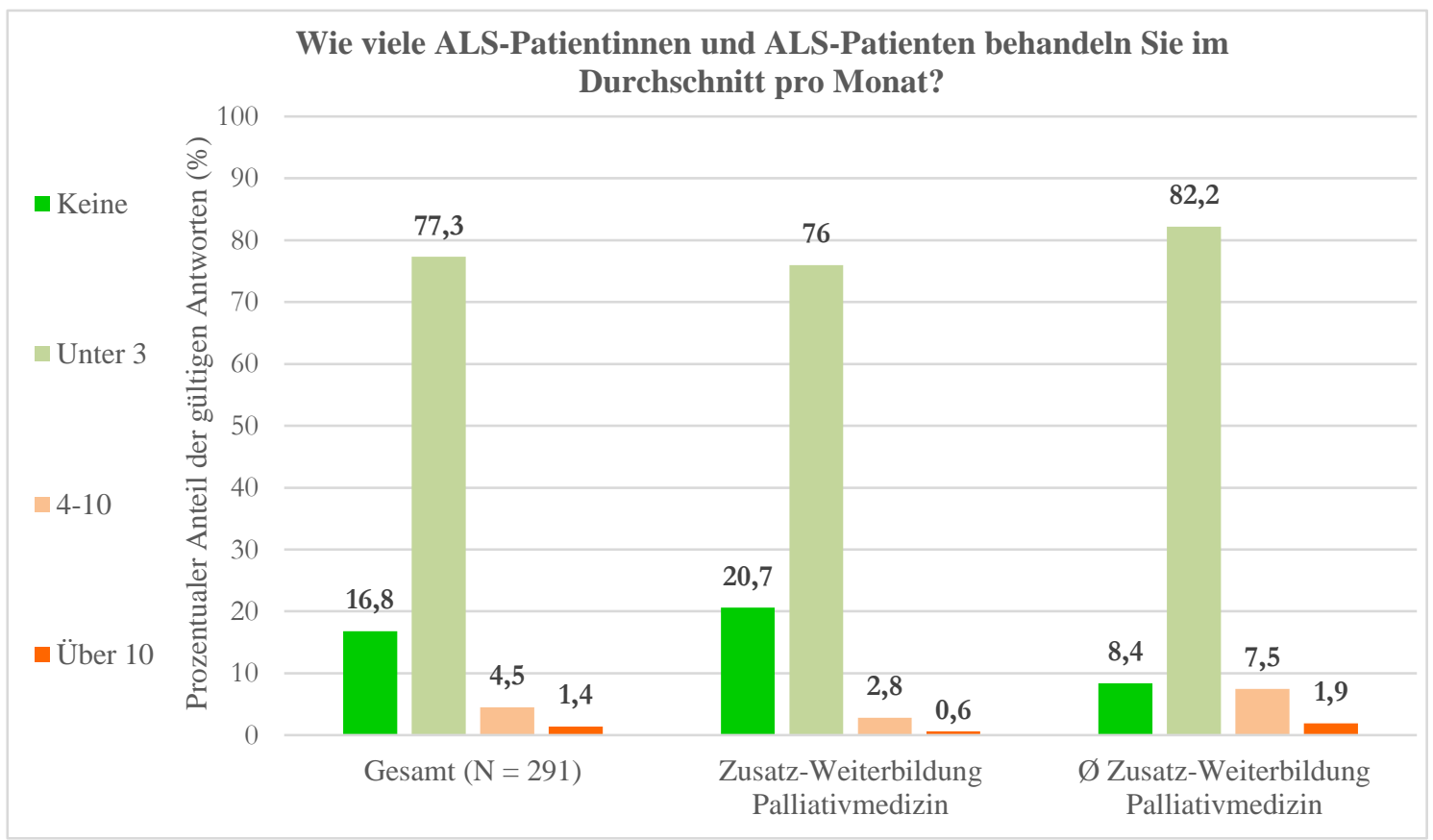

Abbildung 2: Anzahl der behandelten ALS-PatientInnen

Wie in Abbildung 2 zu sehen ist, besteht ein signifikanter Unterschied ( $p=0,00106)$ zwischen Teilnehmenden mit und ohne Zusatz-Weiterbildung Palliativmedizin, wobei ÄrztInnen ohne die Zusatz-Weiterbildung mehr ALS-PatientInnen pro Monat behandeln. Diese Verteilung kann dadurch erklärt werden, dass für die Teilnahme an der Befragung ÄrztInnen mit Weiterbildungsermächtigung Neurologie einerseits und Weiterbildungsermächtigung Palliativmedizin andererseits ausgewählt wurden. Die Gruppe „Ø ZusatzWeiterbildung Palliativmedizin" besteht dementsprechend zum großen Teil aus NeurologInnen, welche primär für die Behandlung von ALS-PatientInnen verantwortlich sind. 
Anschließend wurden die Teilnehmenden gebeten anzugeben, wie lange sie bereits ALSPatientInnen behandeln. Die Ergebnisse sind in Abbildung 3 zusammengefasst. Gruppenübergreifend wiesen $43 \%$ der Antwortenden eine entsprechende Berufserfahrung von über 15 Jahren auf. Etwa ein Drittel gab an, seit 5-15 Jahren ALS-PatientInnen zu behandeln und $11 \%$ hatten zum Zeitpunkt der Befragung keine Erfahrung mit der Behandlung von ALS-PatientInnen.

Es bestand wiederum ein signifikanter Unterschied $(p<0,00001)$ zwischen den teilnehmenden ÄrztInnen mit und ohne Zusatz-Weiterbildung Palliativmedizin. Im Einklang mit der vorangegangenen Frage wiesen die TeilnehmerInnen ohne Zusatz-Weiterbildung eine deutlich längere Erfahrung mit der Behandlung von ALS-PatientInnen auf. Drei Viertel der ÄrztInnen ohne Zusatz-Weiterbildung gaben einen Zeitraum von über 15 Jahren an, während nur $2 \%$ keine Erfahrung mit der Behandlung von ALS-PatientInnen hatten.

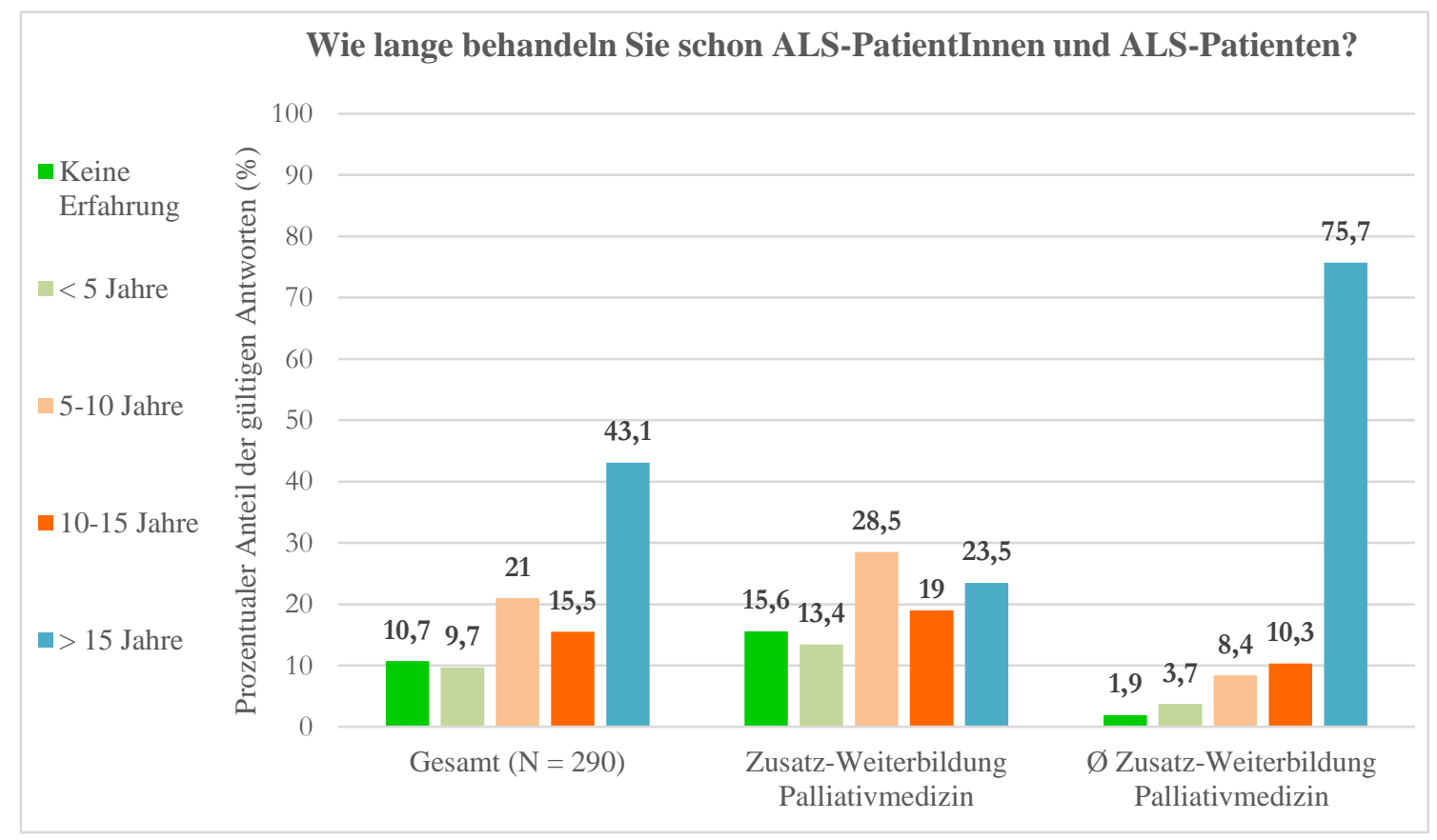

Abbildung 3: Übersicht Erfahrung mit ALS-Behandlung

Ferner bestanden erwartbare signifikante Unterschiede $(\mathrm{p}=0,0001)$ zwischen den Altersgruppen; ein höheres Lebensalter ging mit einer längeren Erfahrung mit der Behandlung von ALS-PatientInnen einher. Zudem wiesen männliche Teilnehmer eine signifikant längere Erfahrung $(\mathrm{p}<0,00001)$ mit der Behandlung von ALS-PatientInnen auf. 
Zum Abschluss des ersten Fragebogenteils wurden die Teilnehmenden gefragt, ob sie bereits eine Palliative Sedierung bei ALS-PatientInnen durchgeführt haben. Eine Übersicht der Ergebnisse ist in Abbildung 4 dargestellt.

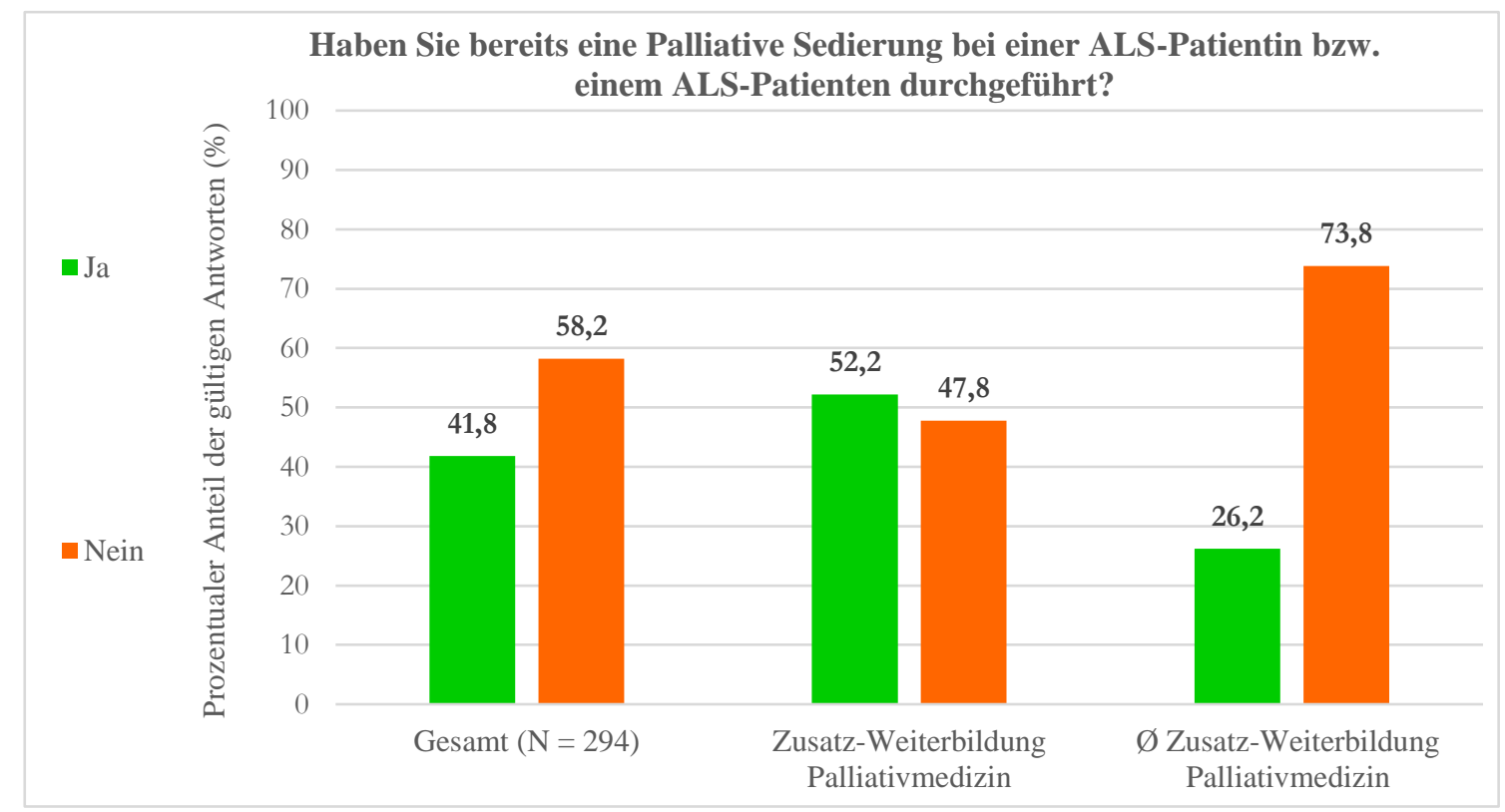

Abbildung 4: Übersicht Erfahrung mit Palliativer Sedierung bei ALS

Insgesamt gaben $42 \%$ der Antwortenden an, bereits eine Palliative Sedierung bei ALSPatientInnen durchgeführt zu haben. Von den Teilnehmenden mit Zusatz-Weiterbildung Palliativmedizin wurde die Frage mit $52 \%$ außerdem signifikant häufiger $(\mathrm{p}=0,00002)$ bejaht als von der Vergleichsgruppe ohne Zusatz-Weiterbildung.

Im Falle einer zustimmenden Antwort, wurden die Teilnehmenden gebeten, im Rahmen einer Freitextantwort die Symptome zu nennen, aufgrund derer die Palliative Sedierung durchgeführt wurde. Insgesamt 123 Personen haben die Freitextfrage beantwortet, wobei Mehrfachnennungen möglich waren, sodass insgesamt 265 Symptome erfasst wurden. Wie Abbildung 5 zeigt, wurden die Freitextantworten in zehn Symptomgruppen zusammengefasst. Eine vollständige Auflistung aller genannten Symptome ist im Anhang zu finden. 
Als Indikation für die Durchführung der Palliativen Sedierung gaben die TeilnehmerInnen insbesondere atmungsbezogene Symptome an. Im Rahmen der Auswertung wurde zwischen subjektiv empfundener Luftnot einerseits und einer eher funktionellen Beschreibung der Ateminsuffizienz andererseits unterschieden. 111 der insgesamt 265 genannten Indikationen konnten diesen atmungsbezogenen Symptomen zugeordnet werden. Als weitere häufige Indikation wurde Angst genannt, gefolgt von Schmerzen und Dysphagie.

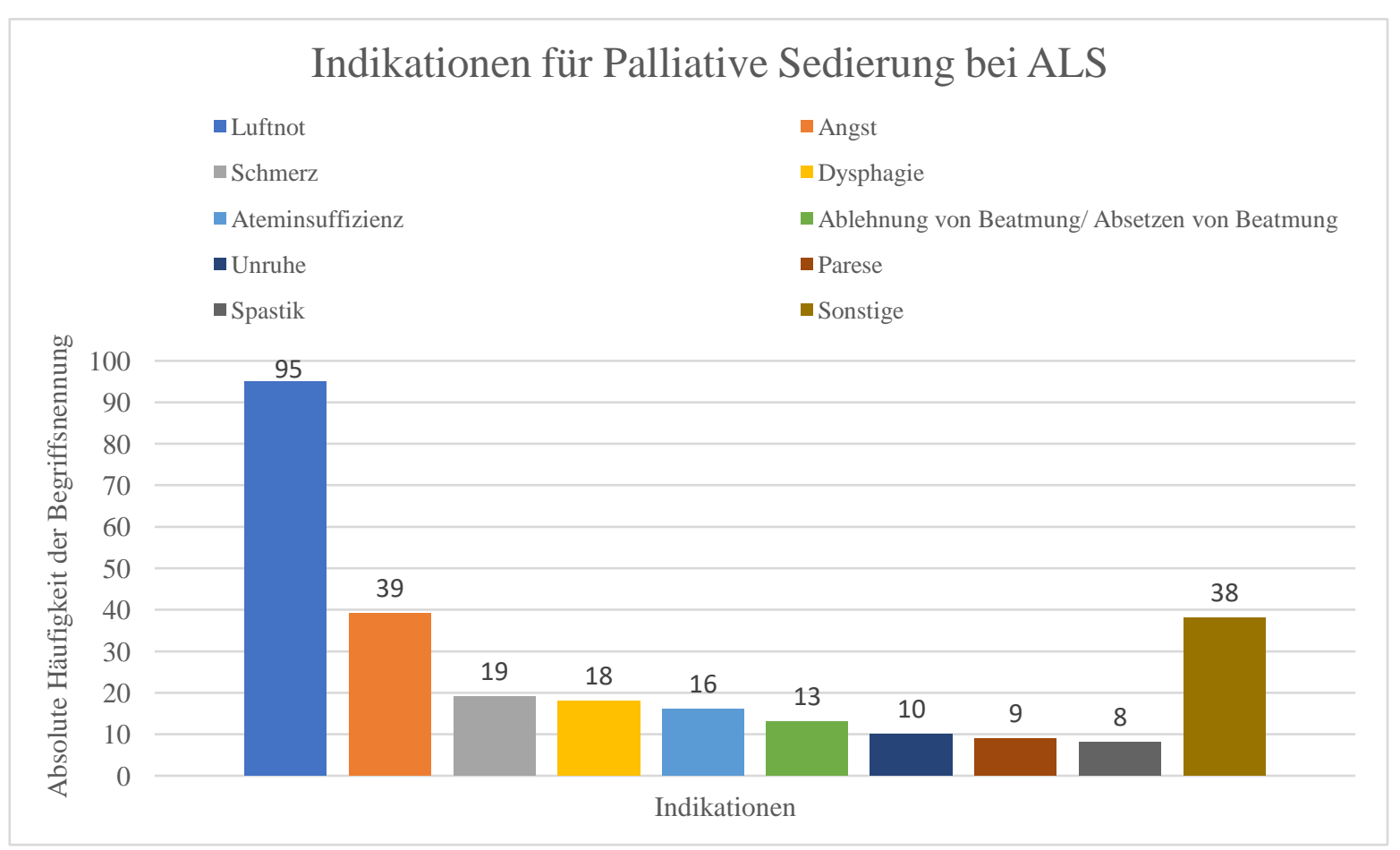

Abbildung 5: Freitextantwort zur Indikation für Palliative Sedierung bei ALS 


\subsection{Fallbeispiel 1.1: Tiefe kontinuierliche Sedierung bei körperlicher Symptomatik}

Im Folgenden werden die Antworten zu den fünf Fallbeispielen (1.1, 1.2, 2.1, 2.2, 3) ausgewertet. Dabei erfolgt zunächst eine Übersicht der Ergebnisse und anschließend eine Darstellung signifikanter Unterschiede hinsichtlich der unabhängigen Variablen „ZusatzWeiterbildung Palliativmedizin“, „Erfahrung mit Palliativer Sedierung von ALS-PatientInnen“ und „Vorherige Beschäftigung mit dem Begriff der Palliativen Sedierung“.

Im Fallbeispiel 1.1 wurde eine ALS-Patientin beschrieben, die aufgrund starker körperlicher Symptomatik eine tiefe und kontinuierliche Palliative Sedierung wünscht. Den TeilnehmerInnen wurden fünf Items präsentiert, die sie mithilfe einer vierstufigen Skala (,Stimme völlig zu“ bis „Stimme nicht zu“) bewerten sollten.

Item 1: Der Wunsch der Patientin ist für mich nachvollziehbar.

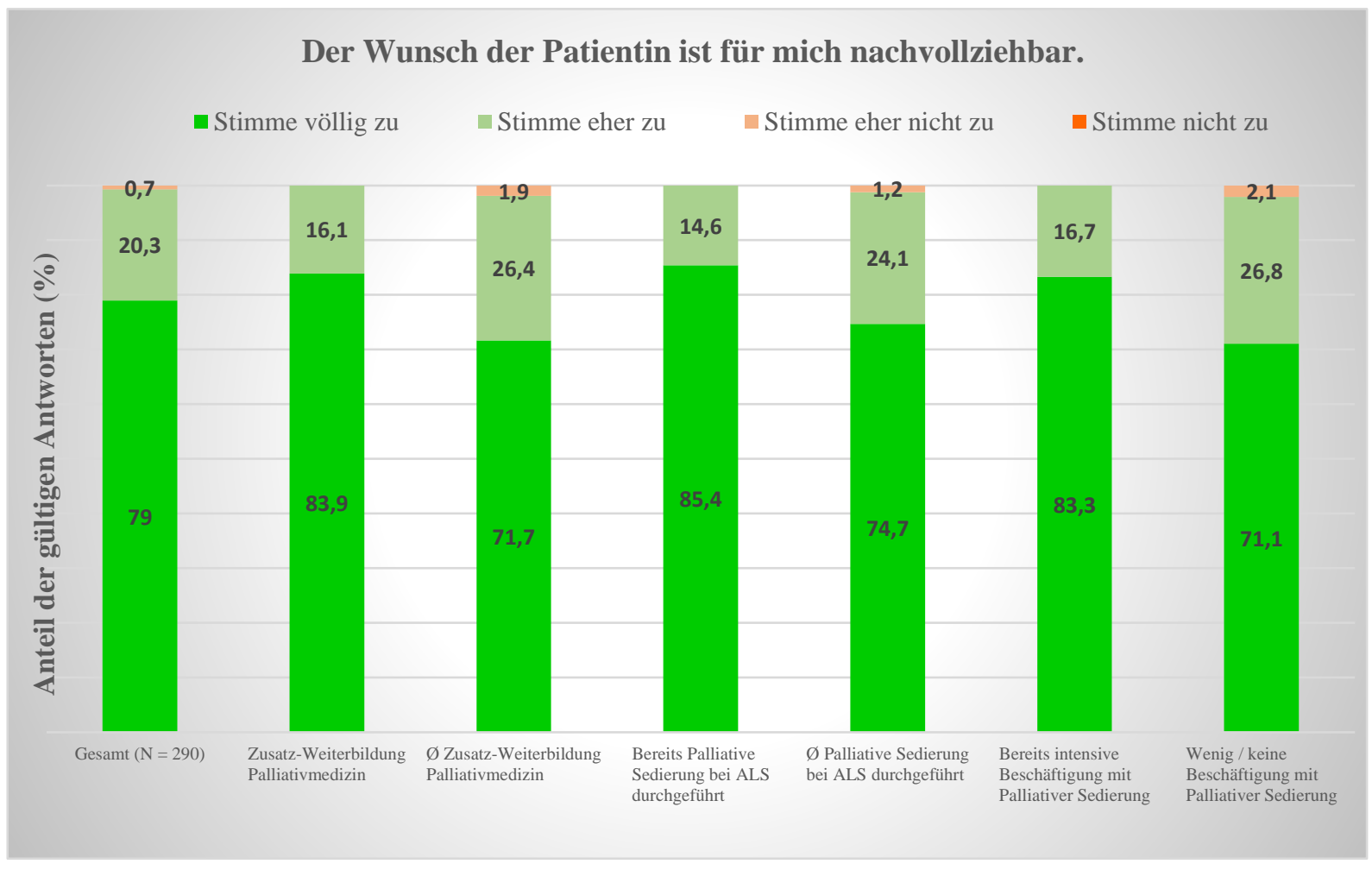

Abbildung 6: Fallbeispiel 1.1, Auswertung Item 1

Insgesamt gaben nahezu $100 \%$ der Antwortenden an, den Wunsch der Patientin nach kontinuierlicher und tiefer Sedierung nachvollziehen zu können. Wie in Abbildung $6 \mathrm{zu}$ sehen ist, zeigte sich ein ähnliches Ergebnis in allen Untergruppen. Zwar sind leichte Gruppenunterschiede sichtbar, diese sind statistisch jedoch nicht signifikant. 


\section{Item 2: Die Patientin hat ein Recht darauf, dass ihrem Wunsch entsprochen wird.}

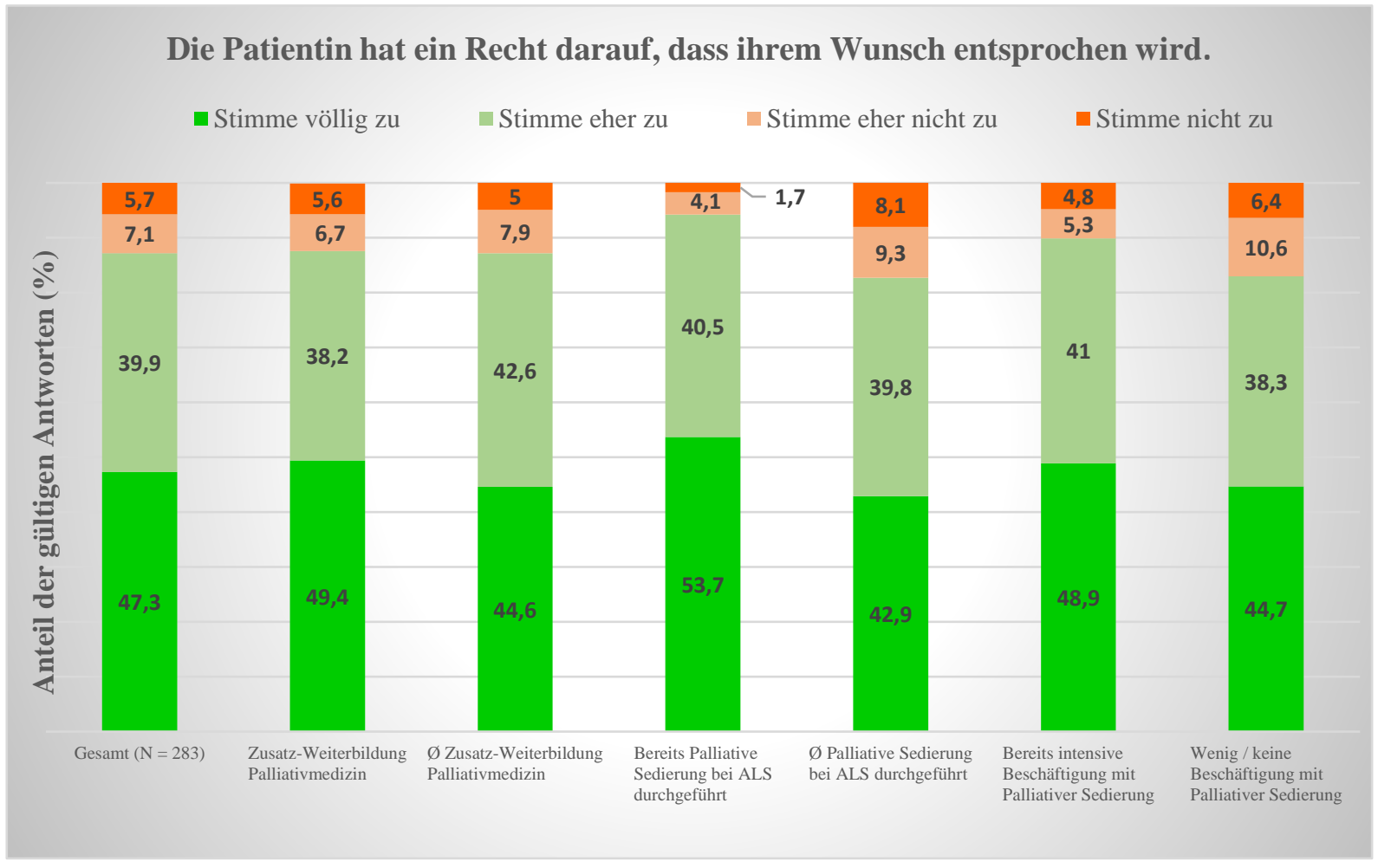

Abbildung 7: Fallbeispiel 1.1, Auswertung Item 2

Gruppenübergreifend stimmten etwa $87 \%$ der Teilnehmenden völlig oder eher zu, dass die Patientin ein Recht auf die Durchführung der gewünschten Maßnahmen habe. Auch hinsichtlich dieses Items sind leichte Gruppenunterschiede erkennbar, die in Abbildung 7 dargestellt sind. Diese sind jedoch nicht statistisch signifikant. 
Item 3: Ich würde dem Wunsch nur nachkommen, wenn vorherige Versuche einer flachen bzw. intermittierenden Sedierung keine ausreichende Symptomlinderung erzielen konnten.

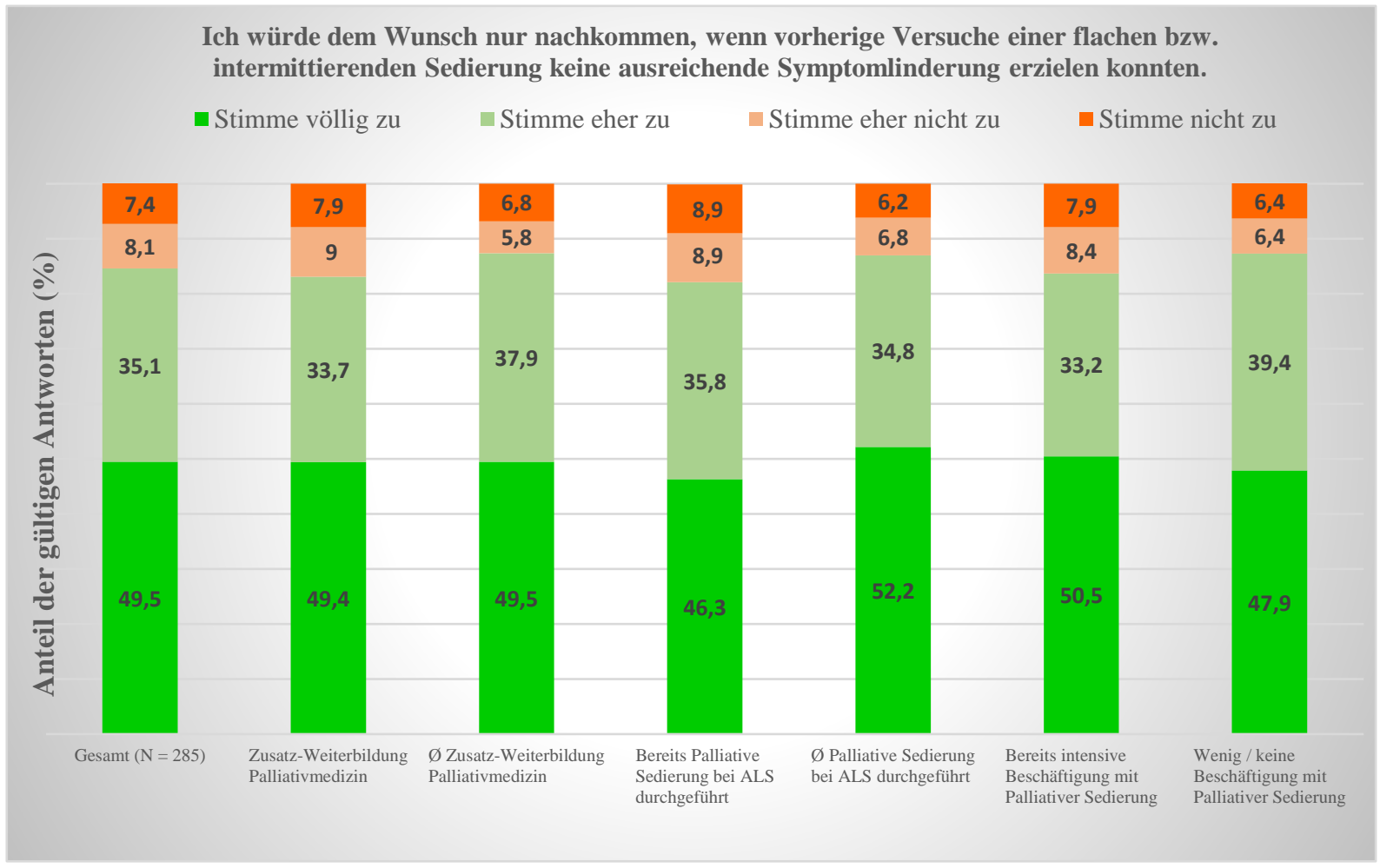

Abbildung 8: Fallbeispiel 1.1, Auswertung Item 3

Mit knapp $85 \%$ würde die Mehrzahl der Antwortenden eine kontinuierliche tiefe Sedierung erst dann durchführen, wenn vorherige Versuche einer flachen oder intermittierenden Sedierung gescheitert sind. Ähnliche Ergebnisse zeigten sich in den verschiedenen Untergruppen. Die detaillierten Ergebnisse sind in Abbildung 8 festgehalten. Hinsichtlich keiner der drei unabhängigen Variablen konnte ein statistisch signifikanter Unterschied festgestellt werden. 
Item 4: Ich würde dem Wunsch nur nachkommen, wenn sich die Patientin bereits unmittelbar in der Sterbephase befindet.

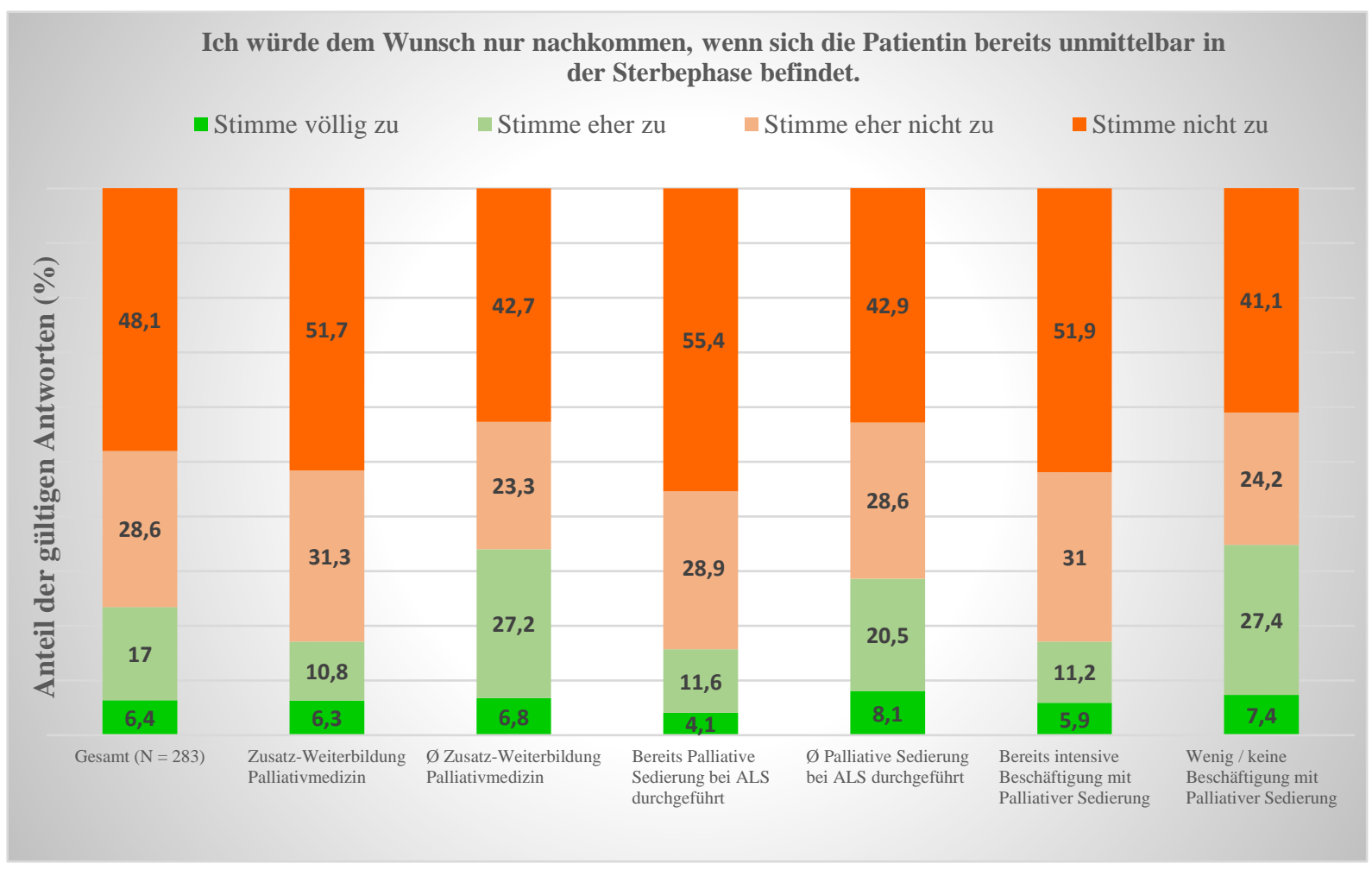

Abbildung 9: Fallbeispiel 1.1, Auswertung Item 4

Über drei Viertel der Antwortenden reagierten ablehnend darauf, eine tiefe kontinuierliche Sedierung nur dann durchzuführen, wenn sich die Patientin bereits unmittelbar in der Sterbephase befindet. Wiederum lagen kleinere Unterschiede zwischen den Untergruppen vor, die in Abbildung 9 graphisch dargestellt sind. Diese sind jedoch nicht statistisch signifikant. 


\section{Item 5: Ich würde generell keine kontinuierliche tiefe Sedierung durchführen.}

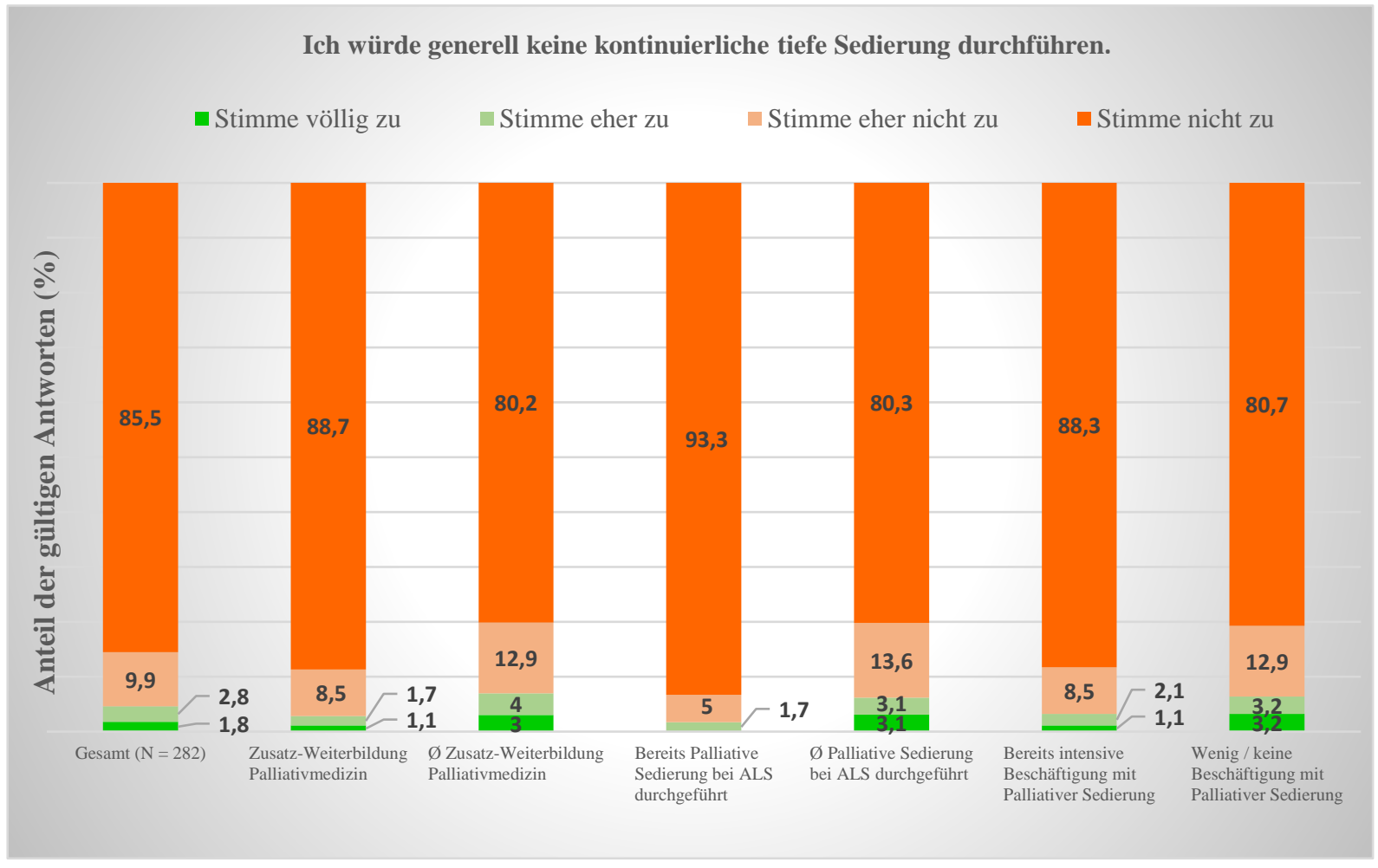

Abbildung 10: Fallbeispiel 1.1, Auswertung Item 5

Insgesamt reagierten $95 \%$ der Antwortenden ablehnend auf die Aussage „Ich würde generell keine kontinuierliche tiefe Sedierung durchführen“. Wie in Abbildung 10 dargestellt, zeigten sich in allen Untergruppen ähnliche Ergebnisse. Es konnten keine statistisch signifikanten Unterschiede hinsichtlich der drei unabhängigen Variablen festgestellt werden. 


\subsection{Fallbeispiel 1.2: Tiefe kontinuierliche Sedierung bei körperlicher Symptomatik mit Verzicht auf künstliche Ernährung}

Fallbeispiel 1.2 unterscheidet sich vom Fallbeispiel 1.1 nur dadurch, dass die Patientin eine künstliche Ernährung während der Sedierung ausdrücklich ablehnt.

Item 1: Der Wunsch der Patientin ist für mich nachvollziehbar.

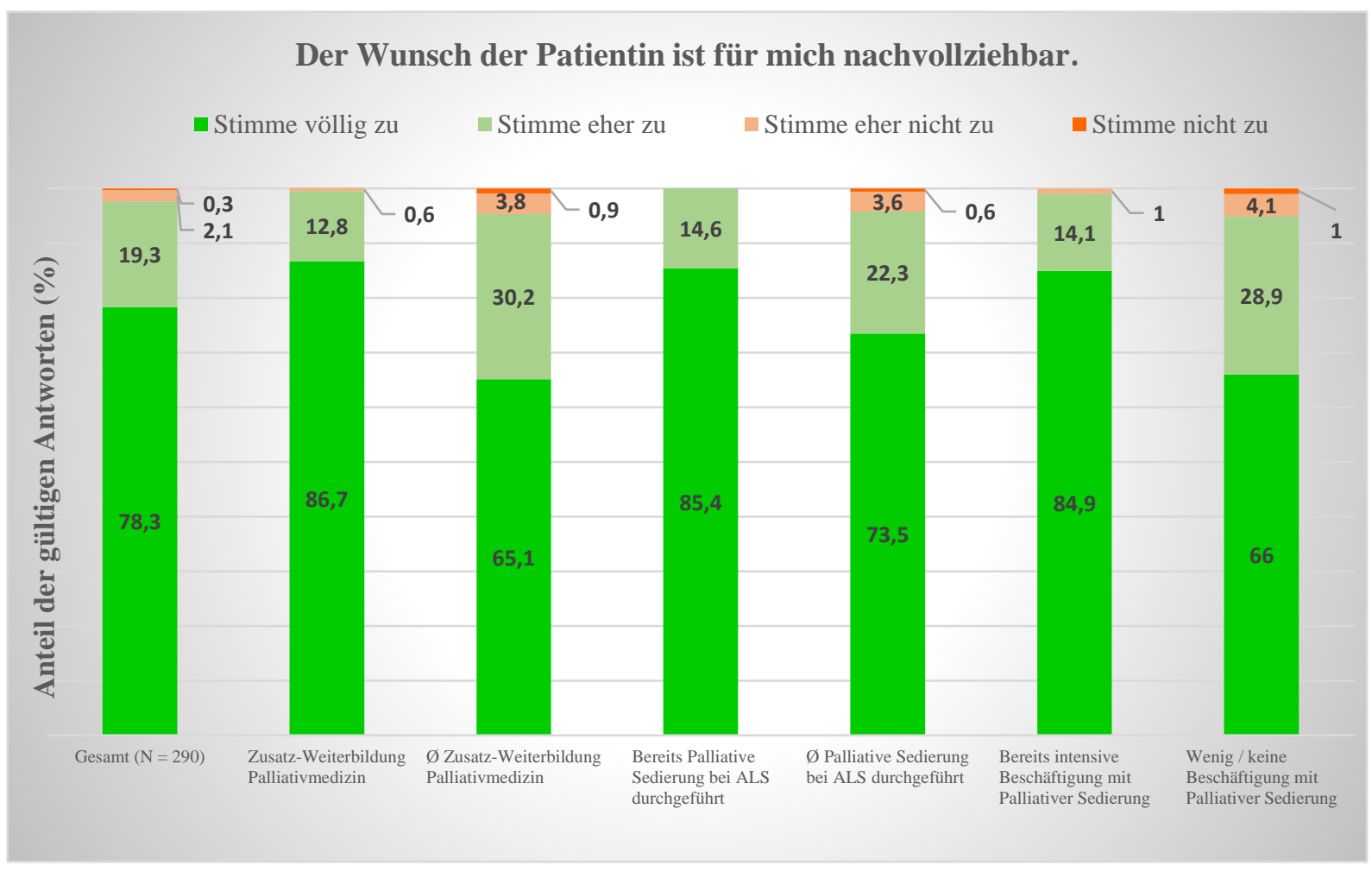

Abbildung 11: Fallbeispiel 1.2, Auswertung Item 1

Insgesamt standen die Teilnehmenden dem Wunsch der Patientin nach kontinuierlicher tiefer Sedierung bei gleichzeitigem Verzicht auf künstliche Ernährung positiv gegenüber. Mit $98 \%$ gab die überwiegende Mehrzahl an, den Wunsch der Patientin völlig oder eher nachvollziehen zu können (siehe Abbildung 11).

Es zeigte sich ein signifikanter Unterschied ( $\mathrm{p}=0,00001)$ zwischen den Teilnehmenden mit und ohne Zusatz-Weiterbildung Palliativmedizin. Zwar konnten in beiden Gruppen über $90 \%$ der Befragten den Wunsch der Patientin nachvollziehen. Die Teilnehmenden mit Zusatz-Weiterbildung antworteten jedoch deutlich häufiger mit „Stimme völlig zu“. Außerdem reagierten die ÄrztInnen, die sich bereits intensiv mit der Thematik beschäftigt hatten, signifikant zustimmender $(p=0,00017)$ als die Vergleichsgruppe. Es zeigte sich kein signifikanter Unterschied zwischen ÄrztInnen mit und ohne praktische Erfahrung mit Palliativer Sedierung von ALS-PatientInnen. 
Item 2: Die Patientin hat ein Recht darauf, dass ihrem Wunsch entsprochen wird.

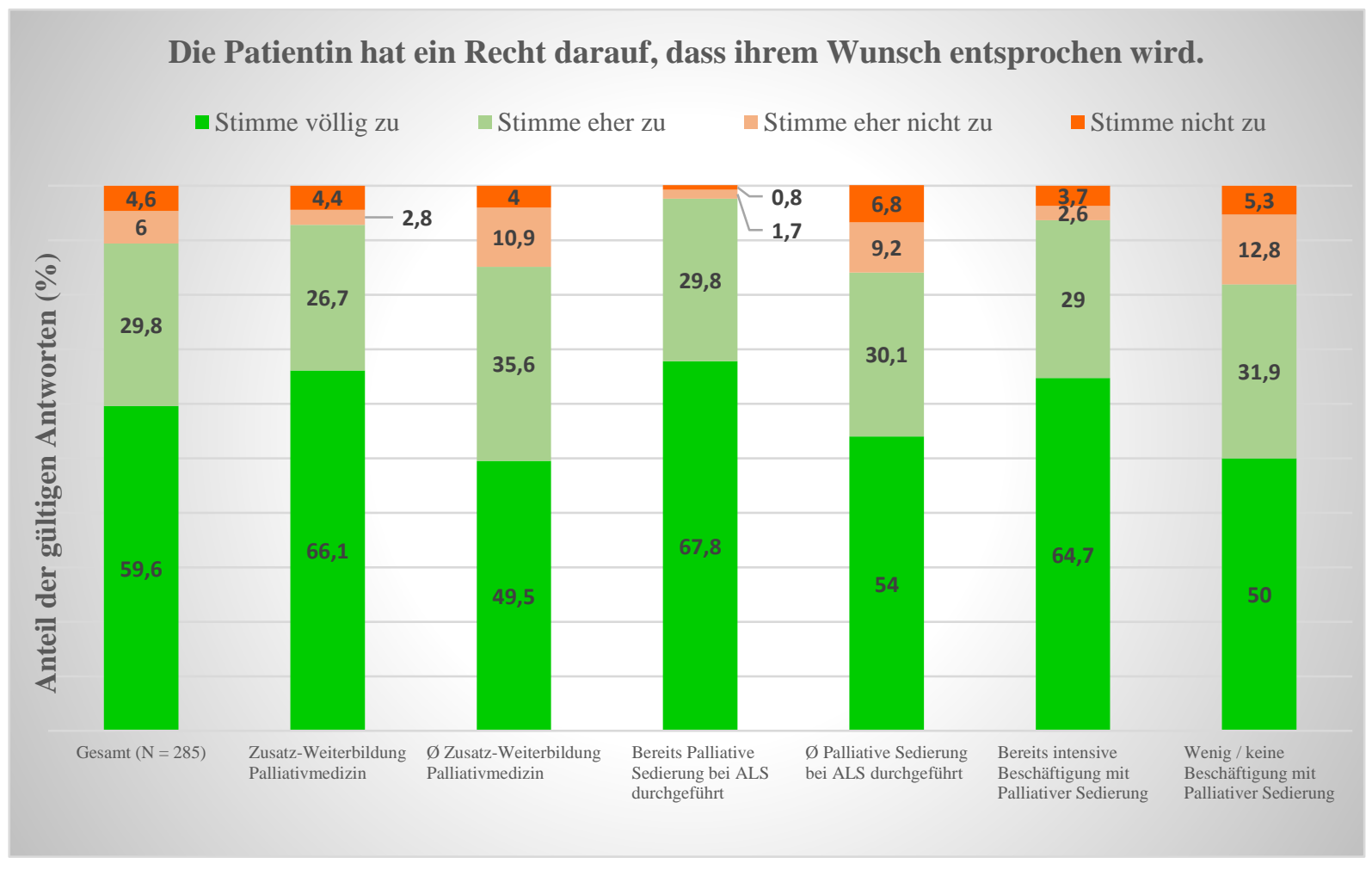

Abbildung 12: Fallbeispiel 1.2, Auswertung Item 2

Insgesamt vertraten $90 \%$ der TeilnehmerInnen die Auffassung, dass die Patientin ein Recht auf die Durchführung der gewünschten Maßnahmen habe. Auch überwogen die zustimmenden Antworten in allen analysierten Untergruppen. Wie in Abbildung 12 dargestellt, bestanden zwar leichte Zustimmungsunterschiede zwischen den Untergruppen, diese sind jedoch nicht statistisch signifikant. 
Item 3: Ich würde dem Wunsch nur nachkommen, wenn dieser in einer Patientenverfügung dokumentiert ist.

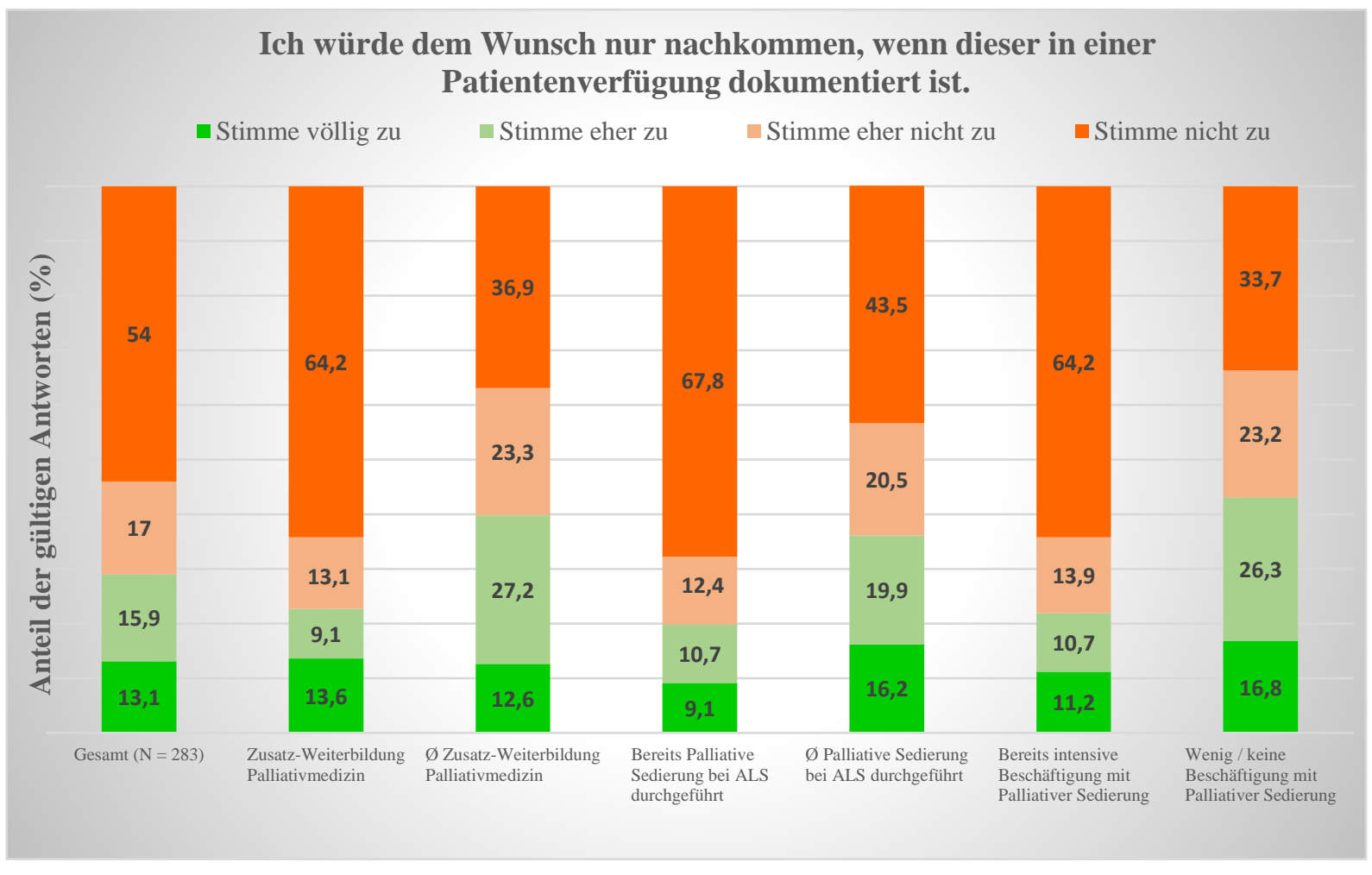

Abbildung 13: Fallbeispiel 1.2, Auswertung Item 3

Knapp zwei Drittel der Antwortenden sahen die Dokumentation des Behandlungswunsches in einer Patientenverfügung nicht als Voraussetzung dafür an, dem Wunsch der Patientin nachzukommen. Es zeigten sich signifikante Gruppenunterschiede sowohl zwischen den Teilnehmenden mit und ohne Zusatz-Weiterbildung Palliativmedizin ( $\mathrm{p}=$ 0,00016) als auch zwischen den Teilnehmenden mit und ohne Erfahrung mit Palliativer Sedierung bei ALS ( $p=0,00011)$ und zwischen den Teilnehmenden mit und ohne vorherige intensive Beschäftigung mit der Thematik ( $\mathrm{p}<0,00001)$.

Wie in Abbildung 13 zu sehen ist, reagierten etwa $75 \%$ der Teilnehmenden mit ZusatzWeiterbildung ablehnend auf das präsentierte Item, während es unter den ÄrztInnen ohne Zusatz-Weiterbildung $60 \%$ waren. Auch stimmten in der Gruppe „Bereits Palliative Sedierung bei ALS durchgeführt" $80 \%$ dem Item nicht oder eher nicht zu. In der Vergleichsgruppe ohne diese praktische Erfahrung waren es hingegen $64 \%$. Unter den Antwortenden, die sich bereits intensiv mit der Thematik beschäftigt hatten, antworteten $78 \%$ ablehnend. Die Vergleichsgruppe mit wenig oder fehlender vorangegangener Beschäftigung mit dem Thema wählte zu 57 \% eine ablehnende Antwort. 


\section{Item 4: Ich würde dem Wunsch nur nachkommen, wenn sich die Patientin bereits unmittelbar in der Sterbephase befindet.}

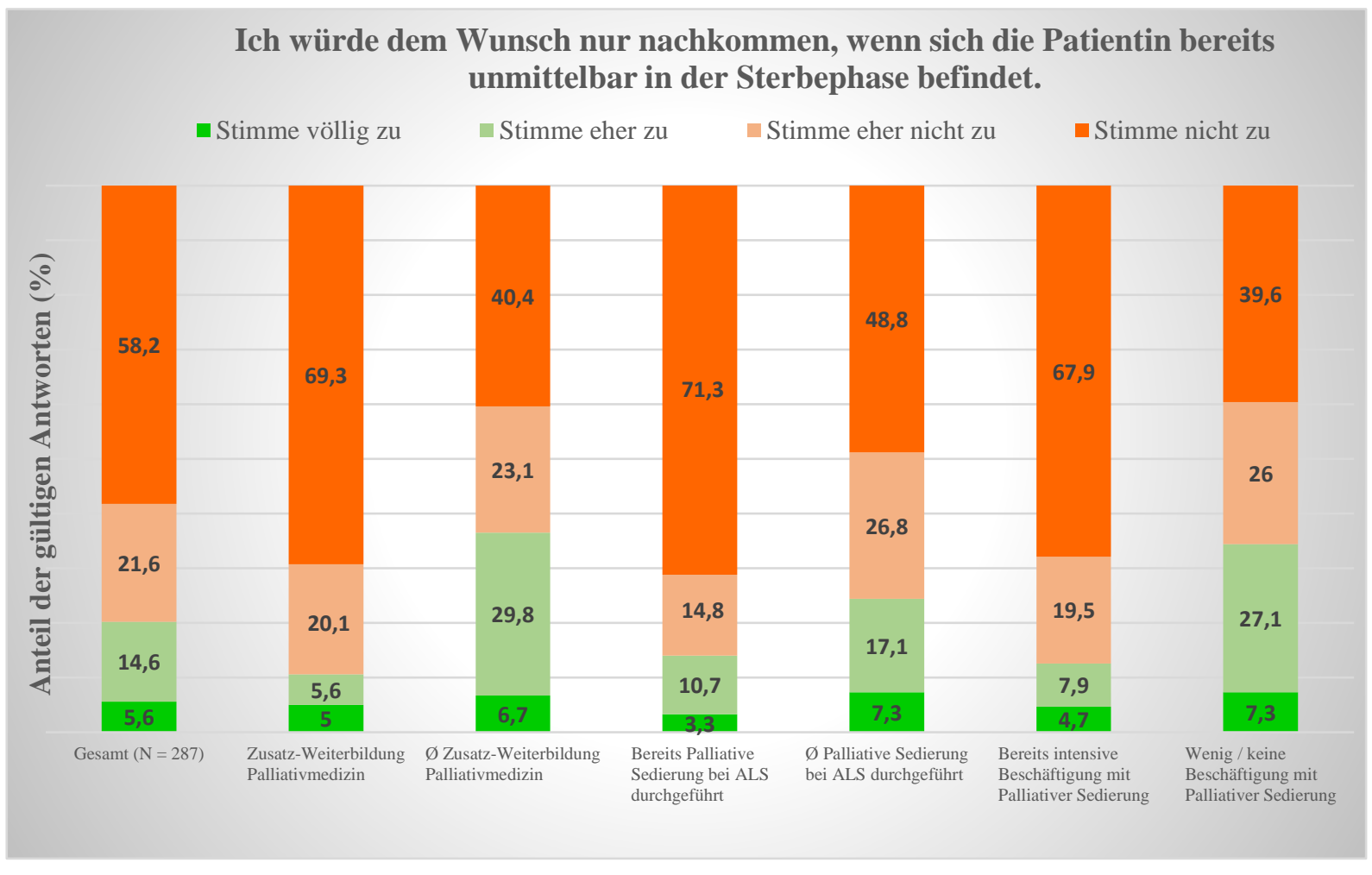

Abbildung 14: Fallbeispiel 1.2, Auswertung Item 4

Gruppenübergreifend lehnten es $80 \%$ der Teilnehmenden ab, dem Wunsch der Patientin erst nachzukommen, wenn sich diese bereits umittelbar in der Sterbephase befindet. Signifikante Gruppenunterschiede zeigten sich sowohl zwischen den Befragten mit und ohne Zusatz-Weiterbildung Palliativmedizin $(\mathrm{p}<0,00001)$ als auch zwischen den Befragten mit und ohne Erfahrung mit der Palliativen Sedierung bei ALS ( $p=0,00023)$ und zwischen Befragten mit und ohne vorherige intensive Beschäftigung mit der Thematik ( $\mathrm{p}$ $0,00001)$.

Knapp $90 \%$ der TeilnehmerInnen mit Zusatz-Weiterbildung Palliativmedizin stimmten gegen eine Begrenzung der gewünschten Maßnahme auf die unmittelbare Sterbephase (siehe Abbildung 14). Unter den ÄrztInnen ohne Zusatz-Weiterbildung antworteten hingegen nur $64 \%$ ablehnend. Innerhalb der Gruppe „Bereits Palliative Sedierung bei ALS durchgeführt" stimmten $86 \%$ dem Item nicht oder eher nicht zu, während es in der Vergleichsgruppe $76 \%$ waren. Zudem zeigte sich, dass eine intensivere vorangegangene Beschäftigung mit der Thematik tendenziell mit einer stärkeren Ablehnung des Items einherging. 


\section{Item 5: Der Verzicht auf künstliche Ernährung bei kontinuierlicher tiefer Sedierung wäre in meinen Augen mit einer Tötung auf Verlangen gleichzusetzen.}

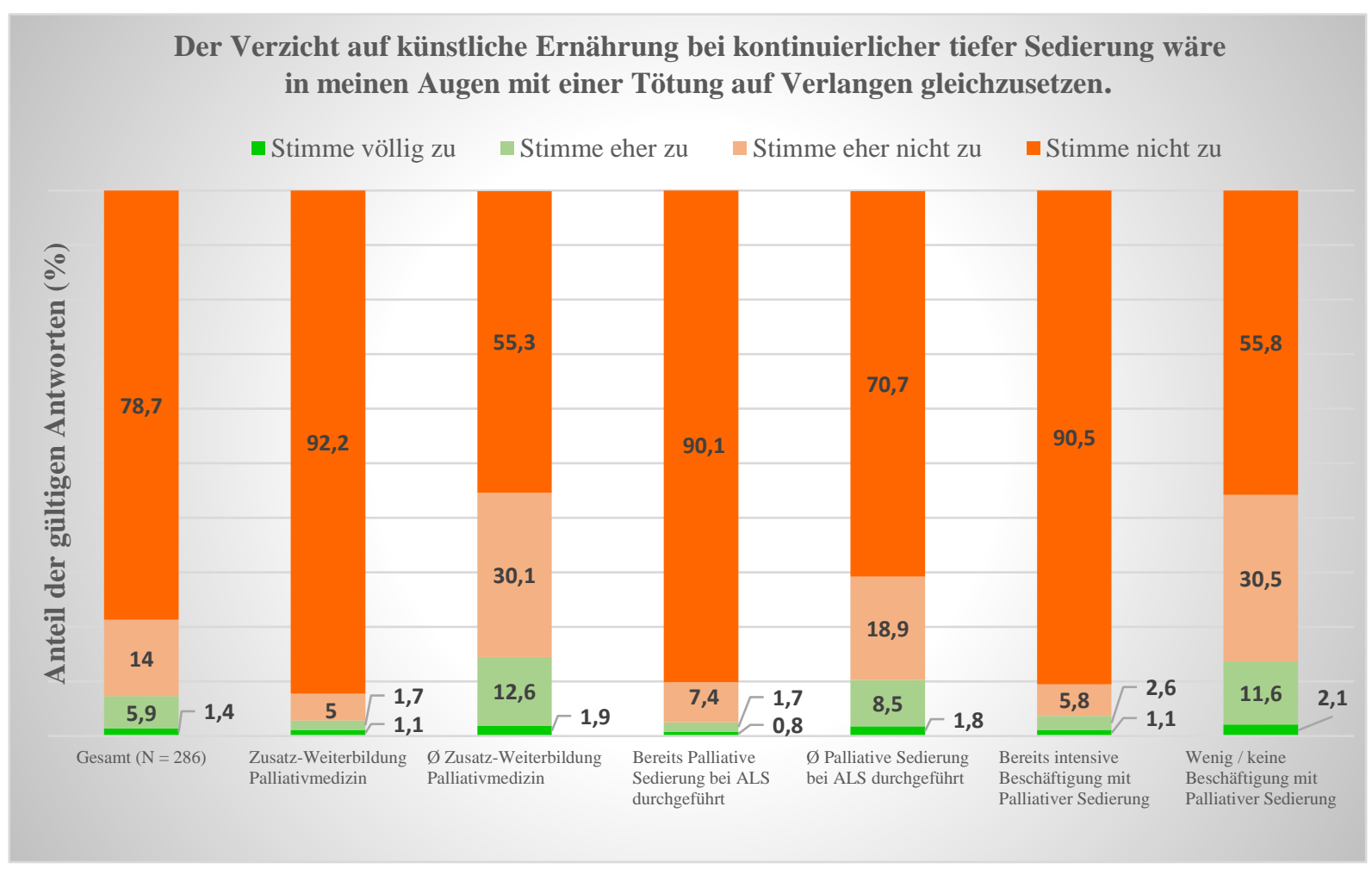

Abbildung 15: Fallbeispiel 1.2, Auswertung Item 5

Insgesamt lehnten $93 \%$ der Antwortenden eine Gleichsetzung der gewünschten Maßnahmen mit einer Tötung auf Verlangen ab. Wiederum zeigten sich signifikante Gruppenunterschiede zwischen den Teilnehmenden mit und ohne Zusatz-Weiterbildung Palliativmedizin $(\mathrm{p}<0,00001)$, zwischen den Teilnehmenden mit und ohne Erfahrung mit der Palliativen Sedierung bei ALS ( $\mathrm{p}=0,00007)$ und zwischen den Teilnehmenden mit und ohne vorherige intensive Beschäftigung mit der Thematik ( $\mathrm{p}<0,00001)$.

Wie in Abbildung $15 \mathrm{zu}$ sehen ist, reagierten etwa $97 \%$ der Antwortenden mit ZusatzWeiterbildung und $85 \%$ der Teilnehmenden ohne Zusatz-Weiterbildung ablehnend auf das präsentierte Item. Außerdem antworteten diejenigen ÄrztInnen, die bereits eine Palliative Sedierung bei ALS durchgeführt hatten, deutlich weniger zustimmend als die entsprechende Vergleichsgruppe. Weiterhin ging eine intensivere vorangegangene Auseinandersetzung mit dem Thema der Palliativen Sedierung tendenziell mit einer stärkeren Ablehnung des Items einher. 
Item 6: Der Verzicht auf künstliche Ernährung bei kontinuierlicher und tiefer Sedierung führt zu einem früheren Versterben als es dem Erkrankungsverlauf ohne diese Maßnahme entsprechen würde.

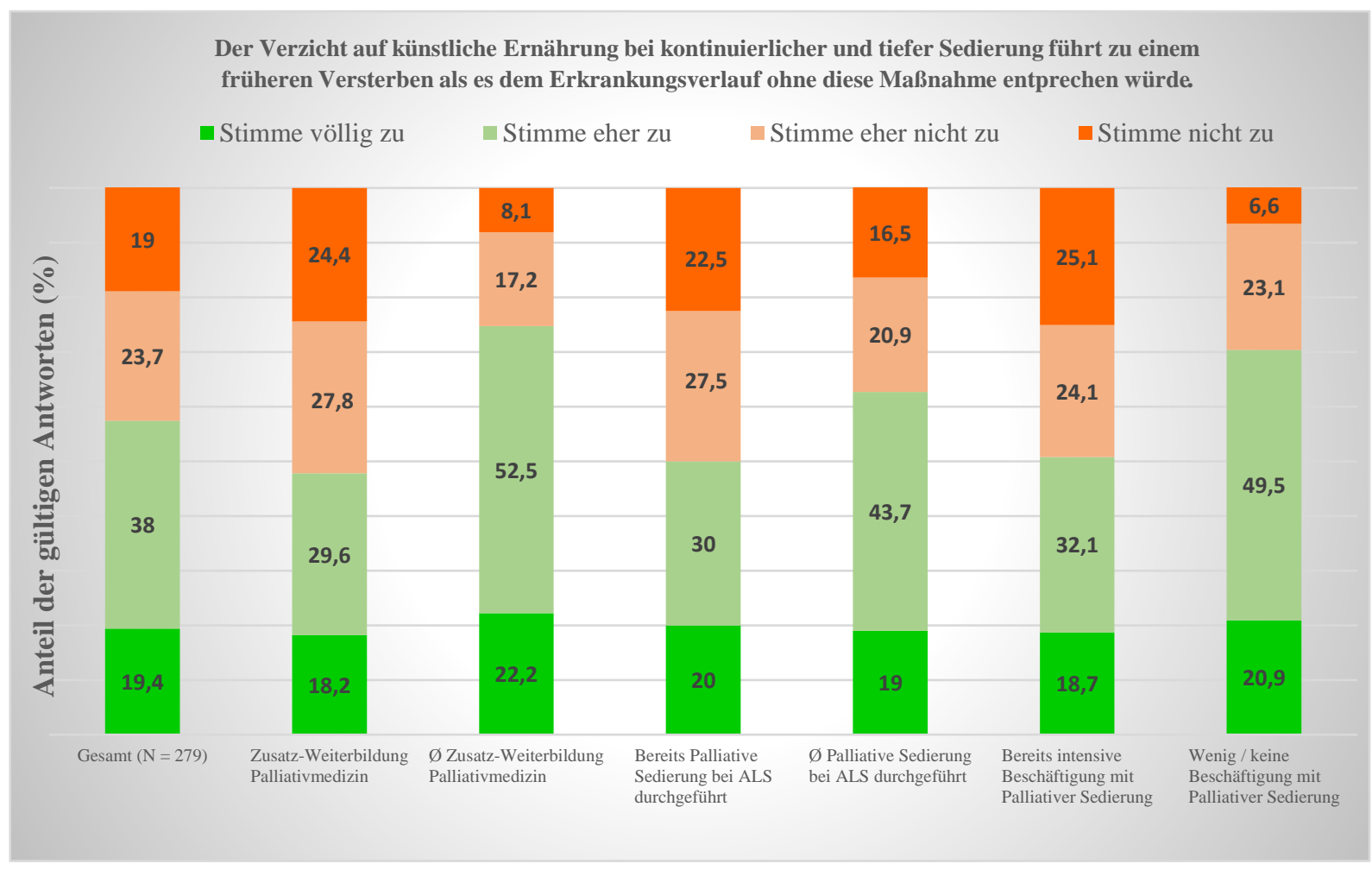

Abbildung 16: Fallbeispiel 1.2, Auswertung Item 6

Gruppenübergreifend vertraten $57 \%$ der Teilnehmenden die Meinung, dass der Verzicht auf künstliche Ernährung bei gleichzeitiger kontinuierlicher tiefer Sedierung zu einem früheren Versterben der Patientin führen würde. Zwischen den Befragten mit und ohne Zusatz-Weiterbildung Palliativmedizin konnten signifikante Gruppenunterschiede festgestellt werden $(\mathrm{p}=0,00019)$.

Innerhalb der Gruppe mit Zusatz-Weiterbildung Palliativmedizin reagierten etwa ebenso viele TeilnehmerInnen zustimmend wie ablehnend auf das präsentierte Item (siehe Abbildung 16). Hingegen antworteten drei Viertel der Antwortenden ohne Zusatz-Weiterbildung völlig oder eher zustimmend. Das Antwortverhalten der übrigen Untergruppen wies keine statistisch signifikanten Unterschiede auf. 


\section{Item 7: Eine künstliche Ernährung bei einer kontinuierlichen tiefen Sedierung wäre in meinen Augen medizinisch nicht indiziert.}

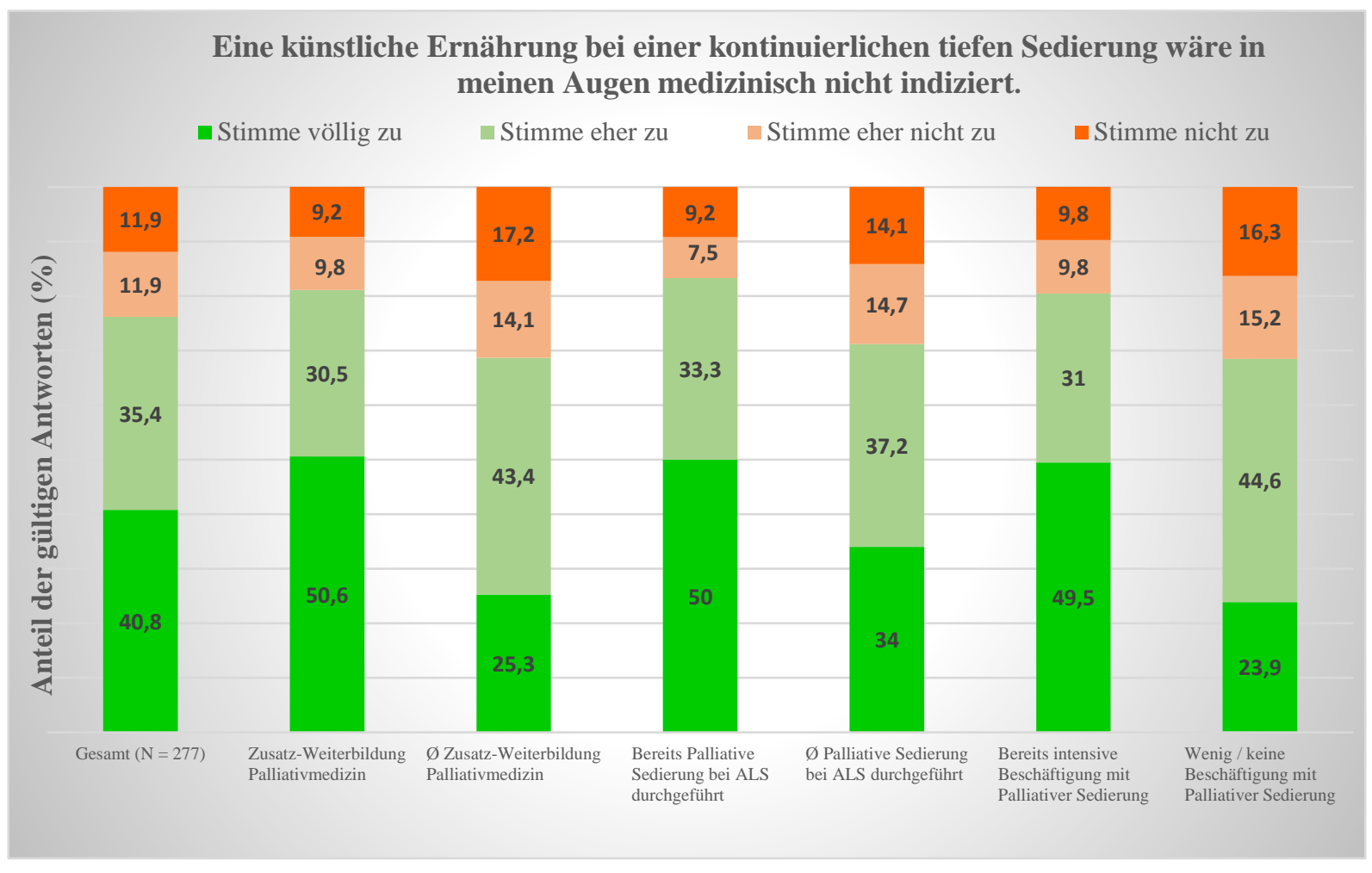

Abbildung 17: Fallbeispiel 1.2, Auswertung Item 7

Insgesamt waren etwa drei Viertel der Teilnehmenden der Ansicht, dass eine künstliche Ernährung während einer dauerhaften tiefen Sedierung nicht indiziert sei. Es zeigten sich signifikante Gruppenunterschiede zwischen den Teilnehmenden mit und ohne ZusatzWeiterbildung Palliativmedizin $(\mathrm{p}=0,00008)$ und zwischen den Teilnehmenden mit und ohne vorherige intensive Beschäftigung mit der Thematik $(\mathrm{p}=0,00012)$.

Wie Abbildung 17 zeigt, stimmten $81 \%$ der Antwortenden mit Zusatz-Weiterbildung Palliativmedizin der präsentierten Aussage völlig oder eher zu. Unter den ÄrztInnen ohne Zusatz-Weiterbildung waren es mit $69 \%$ deutlich weniger. Auch reagierten $81 \%$ der Teilnehmenden, die sich bereits intensiv mit der Thematik beschäftigt hatten, völlig oder eher zustimmend, während es in der Vergleichsgruppe knapp 69 \% waren. Nur hinsichtlich der unabhängigen Variable „Erfahrung mit Palliativer Sedierung von ALS-PatientInnen“ zeigten sich keine signifikanten Gruppenunterschiede. 
Zusatzfrage: Welches Vorgehen würden Sie im Kontext der zuletzt beschriebenen Situation als moralisch belastender empfinden?

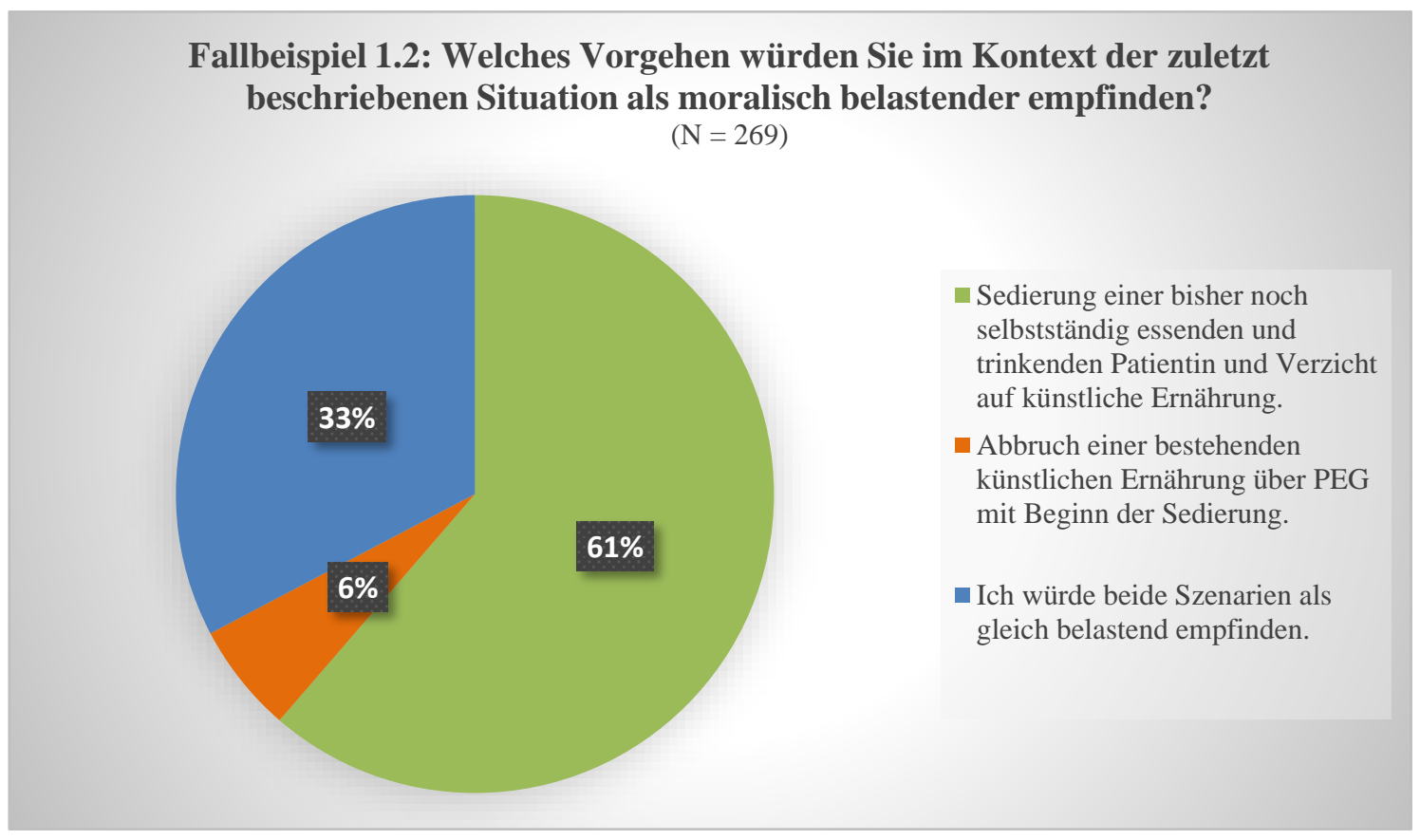

Abbildung 18: Fallbeispiel 1.2, Auswertung Zusatzfrage

Zum Abschluss des Fallbeispiels 1.2 wurden die TeilnehmerInnen gebeten, die in Abbildung 18 dargestellte Zusatzfrage zu beantworten. Es fällt auf, dass dieses Item von 27 der insgesamt 296 Antwortenden nicht bewertet wurde.

Die Mehrheit der Antwortenden stufte die Sedierung einer bisher noch selbstständig essenden und trinkenden Patientin bei gleichzeitigem Verzicht auf künstliche Ernährung als moralisch belastender ein als den Abbruch einer bereits bestehenden künstlichen Ernährung mit Beginn der Sedierung. Ein Drittel der Teilnehmenden bewerteten beide Vorgehensweisen als gleich belastend.

Es zeigten sich keine statistisch signifikanten Unterschiede für die unabhängigen Variablen „Vorherige Beschäftigung mit dem Begriff der Palliativen Sedierung“, „Zusatz-Weiterbildung Palliativmedizin“ und „Erfahrung mit Palliativer Sedierung von ALS-PatientInnen“. 


\subsection{Fallbeispiel 2.1: Tiefe kontinuierliche Sedierung bei psychischer Symptomatik}

Im Fallbeispiel 2.1 wird eine ALS-Patientin beschrieben, die starke psychische Symptome und Belastungen im Sinne existenziellen Leids aufweist. Da sich diese Symptome bisher als therapierefraktär erwiesen haben, wünscht die Patientin eine kontinuierliche tiefe Palliative Sedierung. Die Items, die von den TeilnehmerInnen bewertet werden sollten, sind mit denen des Fallbeispiels 1.1 identisch.

\section{Item 1: Der Wunsch der Patientin ist für mich nachvollziehbar.}

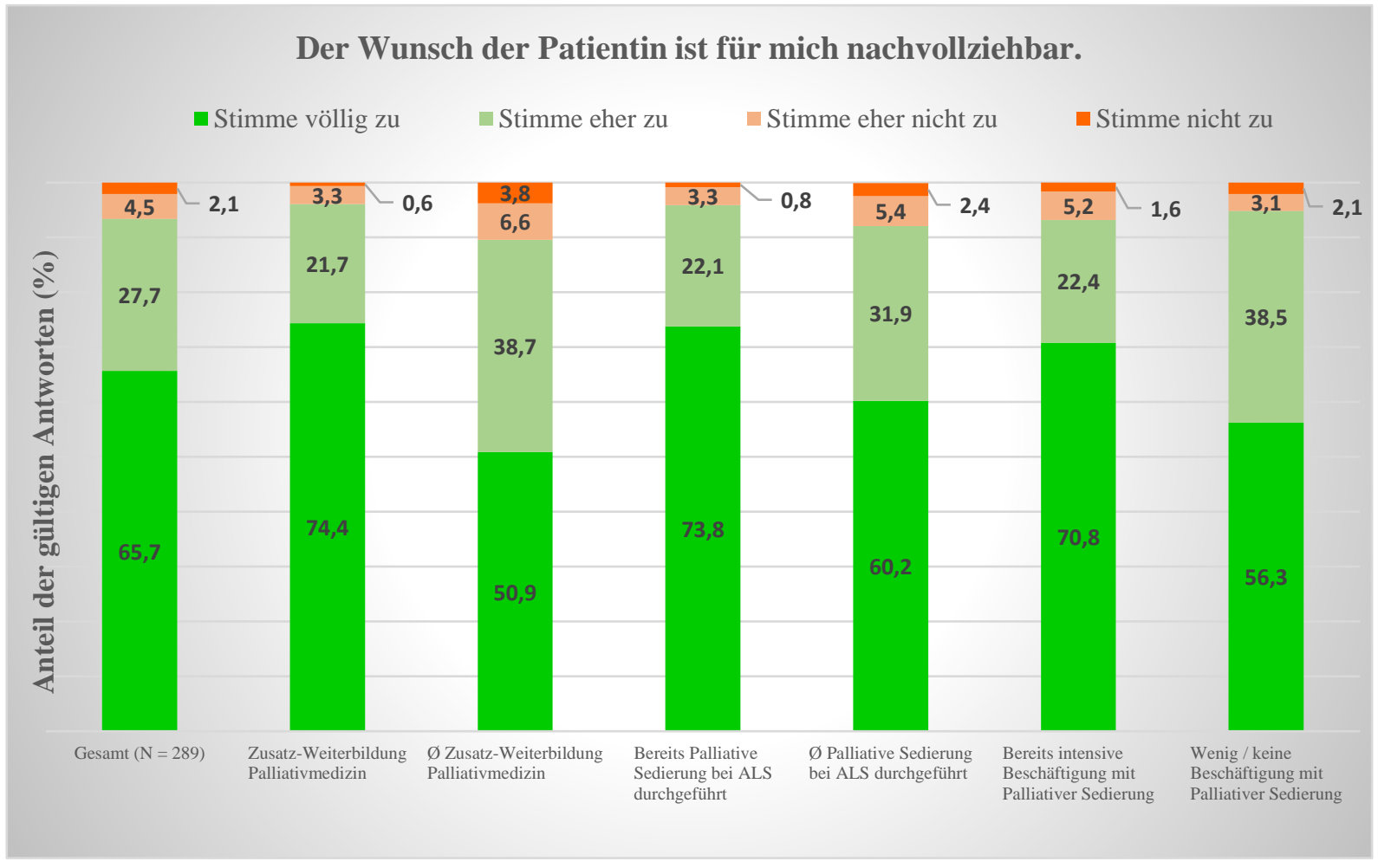

Abbildung 19: Fallbeispiel 2.1, Auswertung Item 1

Mit etwa $93 \%$ der Antwortenden, gab die überwiegende Mehrheit an, den Wunsch der Patientin nachvollziehen zu können. Es zeigten sich signifikante Gruppenunterschiede zwischen den Teilnehmenden mit und ohne Zusatz-Weiterbildung Palliativmedizin ( $\mathrm{p}=$ 0,00004).

Die teilnehmenden ÄrztInnen mit Zusatz-Weiterbildung Palliativmedizin antworteten deutlich zustimmender als die Teilnehmenden ohne Zusatz-Weiterbildung. Wie Abbildung 19 zeigt, bestand dabei der größte Unterschied hinsichtlich der Antwortmöglichkeit „Stimme völlig zu“. Für die unabhängigen Variablen „Vorherige Beschäftigung mit dem Begriff der Palliativen Sedierung“ und „Erfahrung mit Palliativer Sedierung von ALSPatientInnen“ ergaben sich keine statistisch signifikanten Gruppenunterschiede. 


\section{Item 2: Die Patientin hat ein Recht darauf, dass ihrem Wunsch entsprochen wird.}

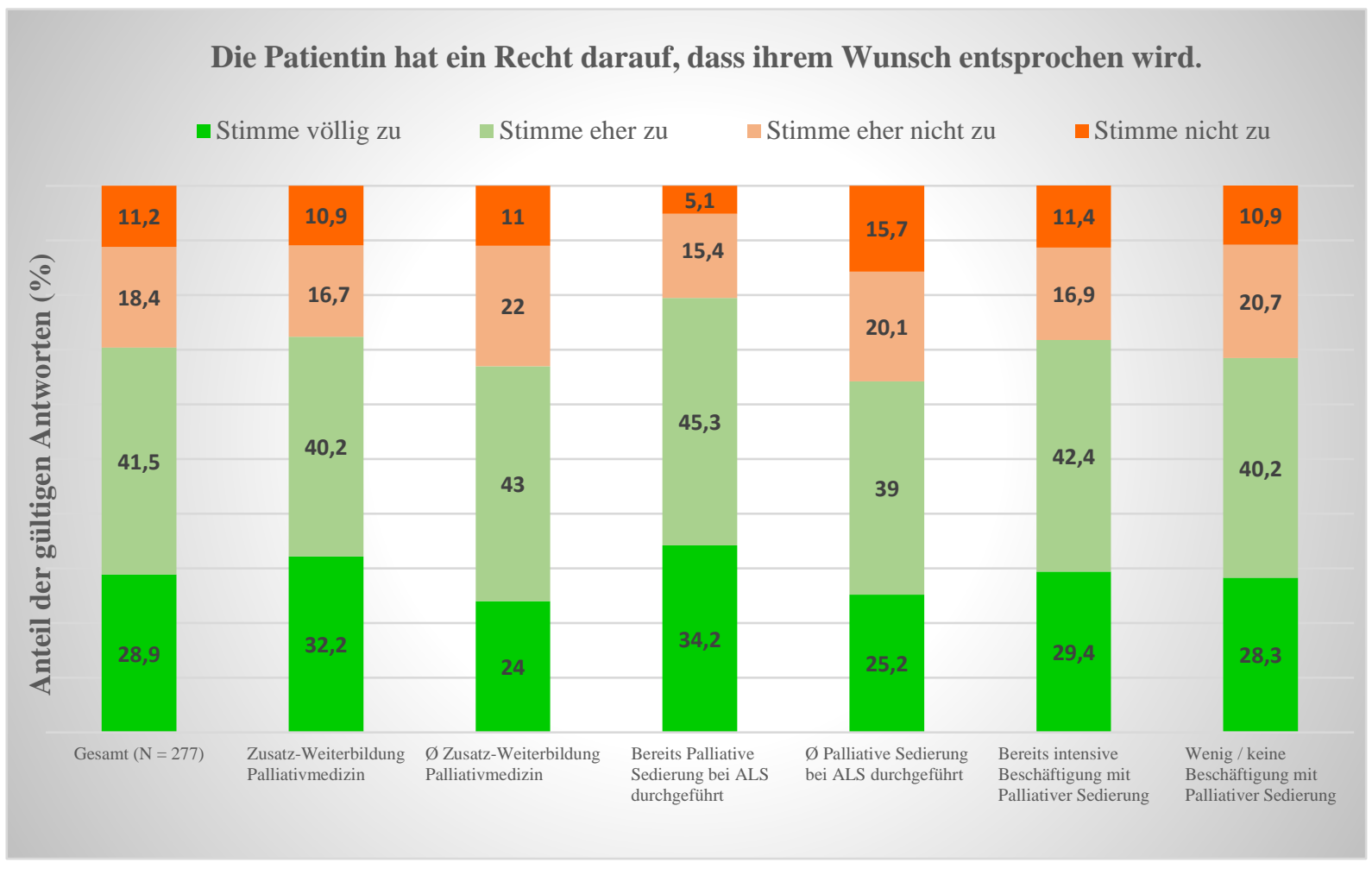

Abbildung 20: Fallbeispiel 2.1, Auswertung Item 2

Gruppenübergreifend stimmten etwa $70 \%$ der Teilnehmenden völlig oder eher zu, dass die Patientin ein Recht darauf habe, dass ihrem Wunsch entsprochen wird. Abbildung 20 zeigt die Gruppenunterschiede im Antwortverhalten. Es konnten jedoch keine statistisch signifikanten Gruppenunterschiede ermittelt werden. 
Item 3: Ich würde dem Wunsch nur nachkommen, wenn vorherige Versuche einer flachen bzw. intermittierenden Sedierung keine ausreichende Symptomlinderung erzielen konnten.

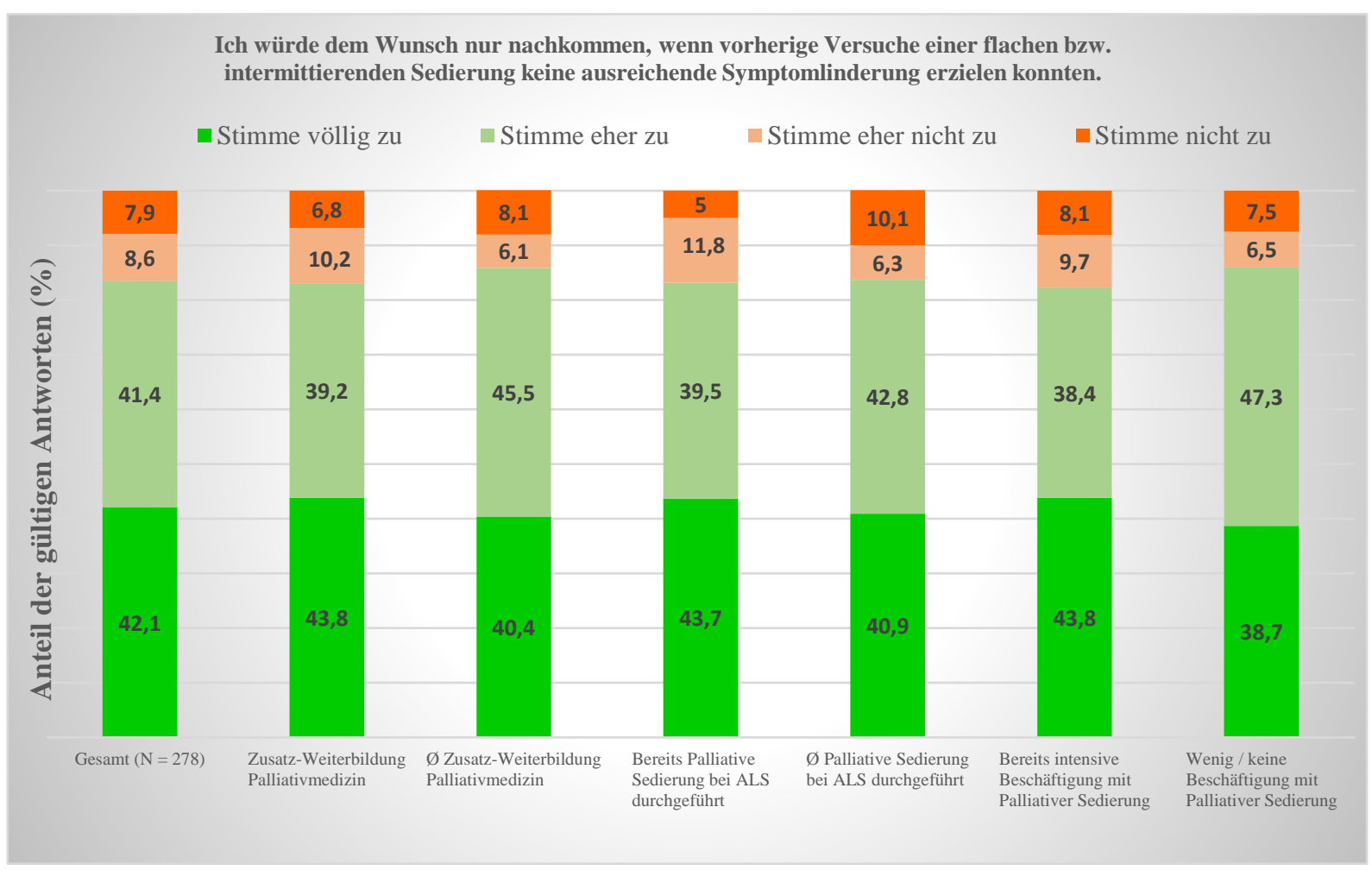

Abbildung 21: Fallbeispiel 2.1, Auswertung Item 3

Insgesamt würden knapp $84 \%$ der Antwortenden dem Wunsch der Patientin erst nachkommen, wenn vorherige Versuche einer flachen oder intermittierenden Sedierung nicht erfolgreich waren. Ähnliche Ergebnisse zeigten sich in allen Untergruppen, wie in Abbildung $21 \mathrm{zu}$ sehen ist. Es konnte für keine der unabhängigen Variablen ein statistisch signifikanter Gruppenunterschied nachgewiesen werden. 


\section{Item 4: Ich würde dem Wunsch nur nachkommen, wenn sich die Patientin bereits unmittelbar in der Sterbephase befindet.}

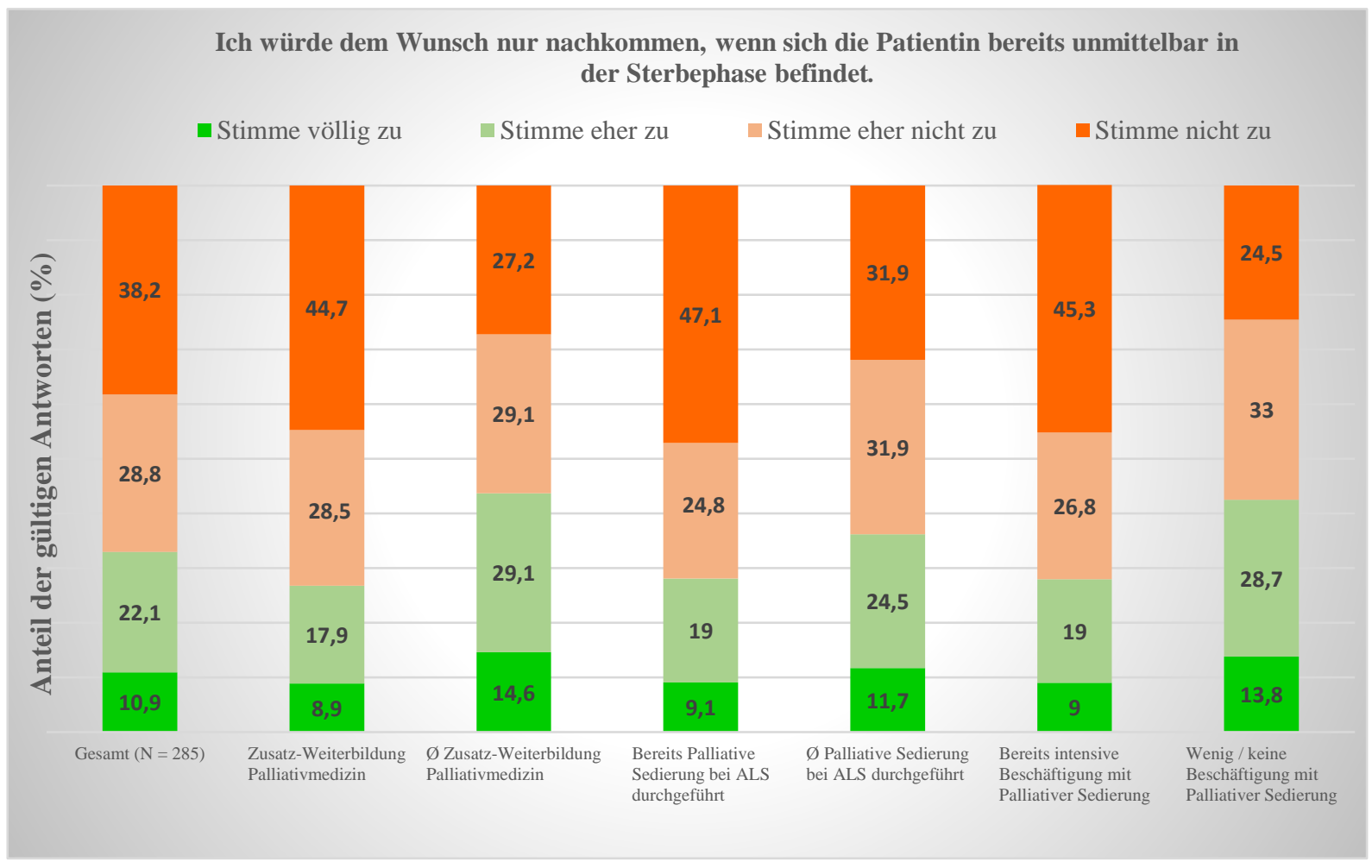

Abbildung 22: Fallbeispiel 2.1, Auswertung Item 4

Gruppenübergreifend lehnten zwei Drittel der Teilnehmenden eine Beschränkung der kontinuierlichen tiefen Sedierung auf die unmittelbare Sterbephase ab. Es zeigten sich signifikante Gruppenunterschiede zwischen den Befragten mit und ohne Zusatz-Weiterbildung Palliativmedizin ( $\mathrm{p}=0,00116)$ und zwischen den Befragten mit und ohne vorherige intensive Beschäftigung mit der Thematik $(p=0,0009)$.

Innerhalb der Gruppe mit Zusatz-Weiterbildung Palliativmedizin reagierten $73 \%$ der Antwortenden ablehnend auf die präsentierte Aussage (siehe Abbildung 22). Unter den ÄrztInnen ohne Zusatz-Weiterbildung waren es hingegen nur $56 \%$. Die Teilnehmenden, die sich bereits intensiv mit der Thematik beschäftigt hatten, lehnten das Item deutlich häufiger ab als die Antwortenden mit wenig oder fehlenden Vorkenntnissen. Bezüglich der Variable „Erfahrung mit Palliativer Sedierung von ALS-PatientInnen“ ergaben sich keine statistisch signifikanten Gruppenunterschiede. 


\section{Item 5: Ich würde generell keine kontinuierliche tiefe Sedierung durchführen.}

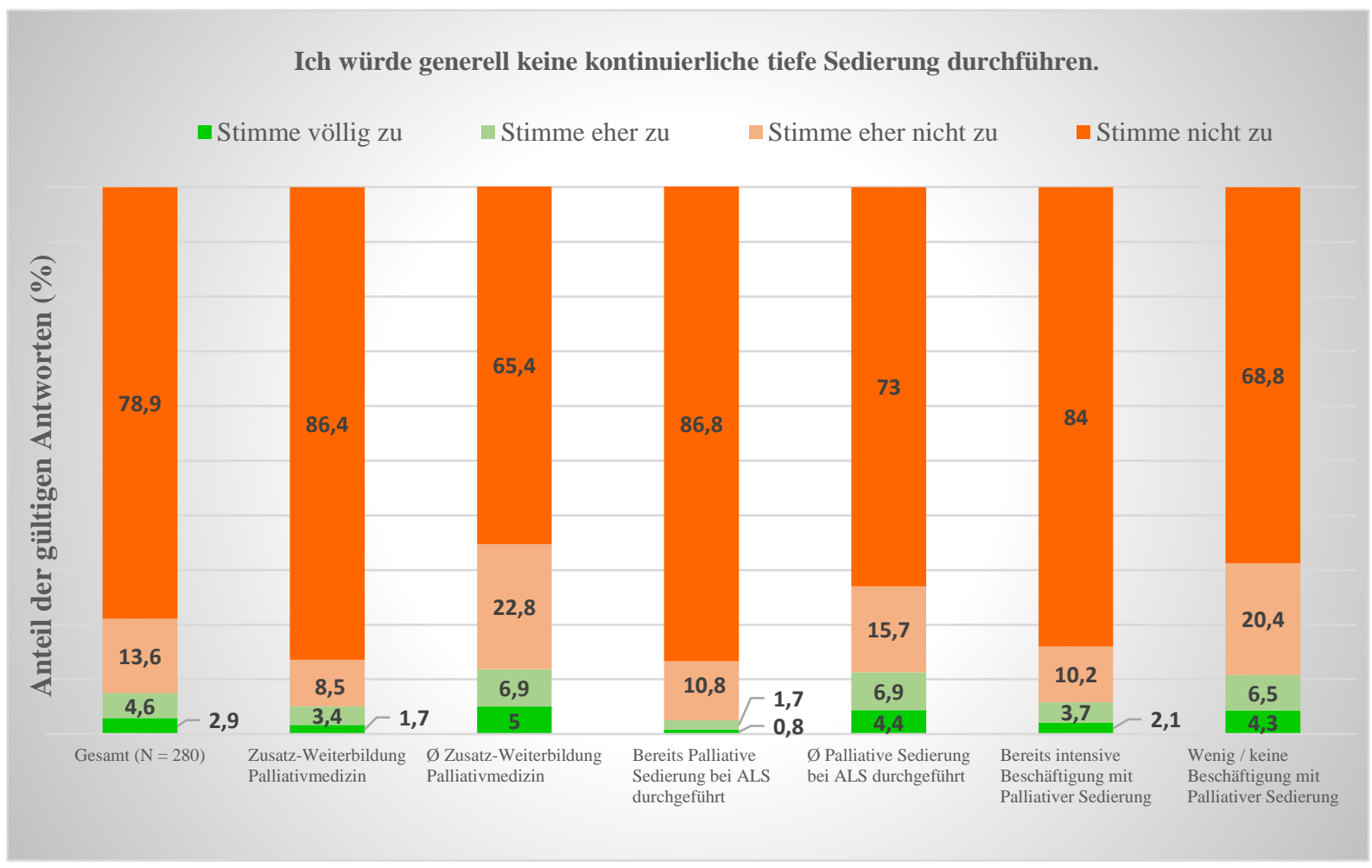

Abbildung 23: Fallbeispiel 2.1, Auswertung Item 5

Knapp $93 \%$ der Antwortenden reagierten ablehnend auf die Aussage „Ich würde generell keine kontinuierliche tiefe Sedierung durchführen“. Ebenso wurde die Aussage in allen Untergruppen von der überwiegenden Mehrzahl der Teilnehmenden abgelehnt. Die detaillierten Ergebnisse sind in Abbildung 23 festgehalten.

Ein statistisch signifikanter Gruppenunterschied zeigte sich nur zwischen den TeilnehmerInnen mit und ohne Zusatz-Weiterbildung Palliativmedizin ( $\mathrm{p}=0,00005)$. Während $86 \%$ der Antwortenden mit Zusatz-Weiterbildung mit „Stimme nicht zu“ antworteten, waren es in der Vergleichsgruppe nur $65 \%$. 


\subsection{Fallbeispiel 2.2: Tiefe kontinuierliche Sedierung bei psychischer Symptomatik mit Verzicht auf künstliche Ernährung}

Fallbeispiel 2.2 unterscheidet sich vom Fallbeispiel 2.1 dadurch, dass die Patientin nun zusätzlich zu einer kontinuierlichen tiefen Sedierung auch einen Verzicht auf künstliche Ernährung während der Sedierung wünscht. Die zu bewertenden Item sind mit denen des Fallbeispiels 1.2 identisch.

Item 1: Der Wunsch der Patientin ist für mich nachvollziehbar.

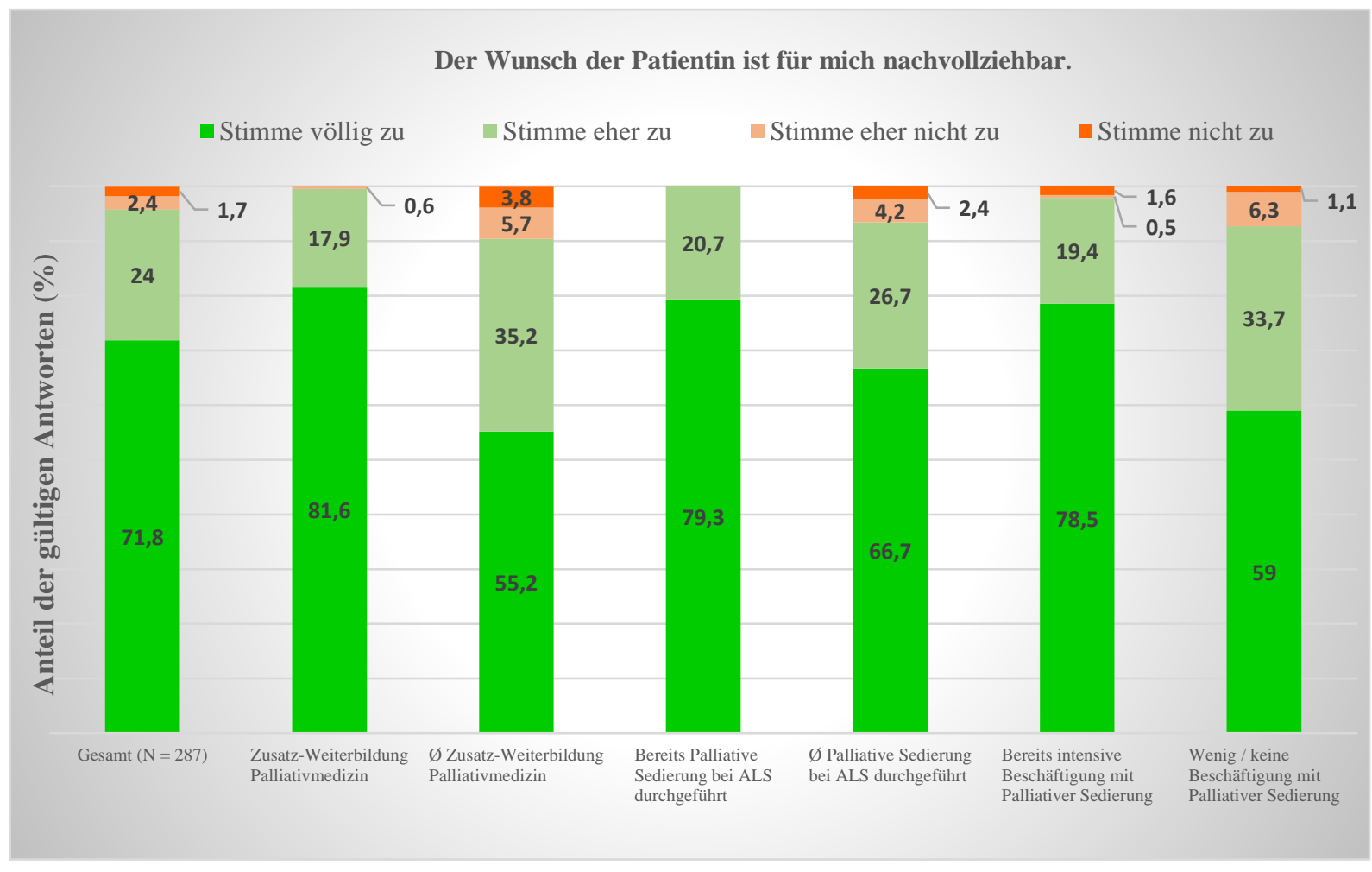

Abbildung 24: Fallbeispiel 2.2, Auswertung Item 1

Mit knapp 96 \% konnte die überwiegende Mehrheit der Antwortenden den Wunsch der Patientin nachvollziehen. Es zeigten sich signifikante Gruppenunterschiede zwischen den Teilnehmenden mit und ohne Zusatz-Weiterbildung Palliativmedizin ( $\mathrm{p}<0,00001)$ und zwischen den Teilnehmenden mit und ohne vorherige intensive Beschäftigung mit der Thematik $(\mathrm{p}=0,00038)$.

Die teilnehmenden ÄrztInnen mit Zusatz-Weiterbildung Palliativmedizin antworteten mit fast $100 \%$ häufiger zustimmend als die Vergleichsgruppe ohne Zusatz-Weiterbildung (siehe Abbildung 24). Auch ging eine intensivere vorangegangene Beschäftigung mit der Thematik tendenziell mit einer größeren Zustimmung einher. 
Item 2: Die Patientin hat ein Recht darauf, dass ihrem Wunsch entsprochen wird.

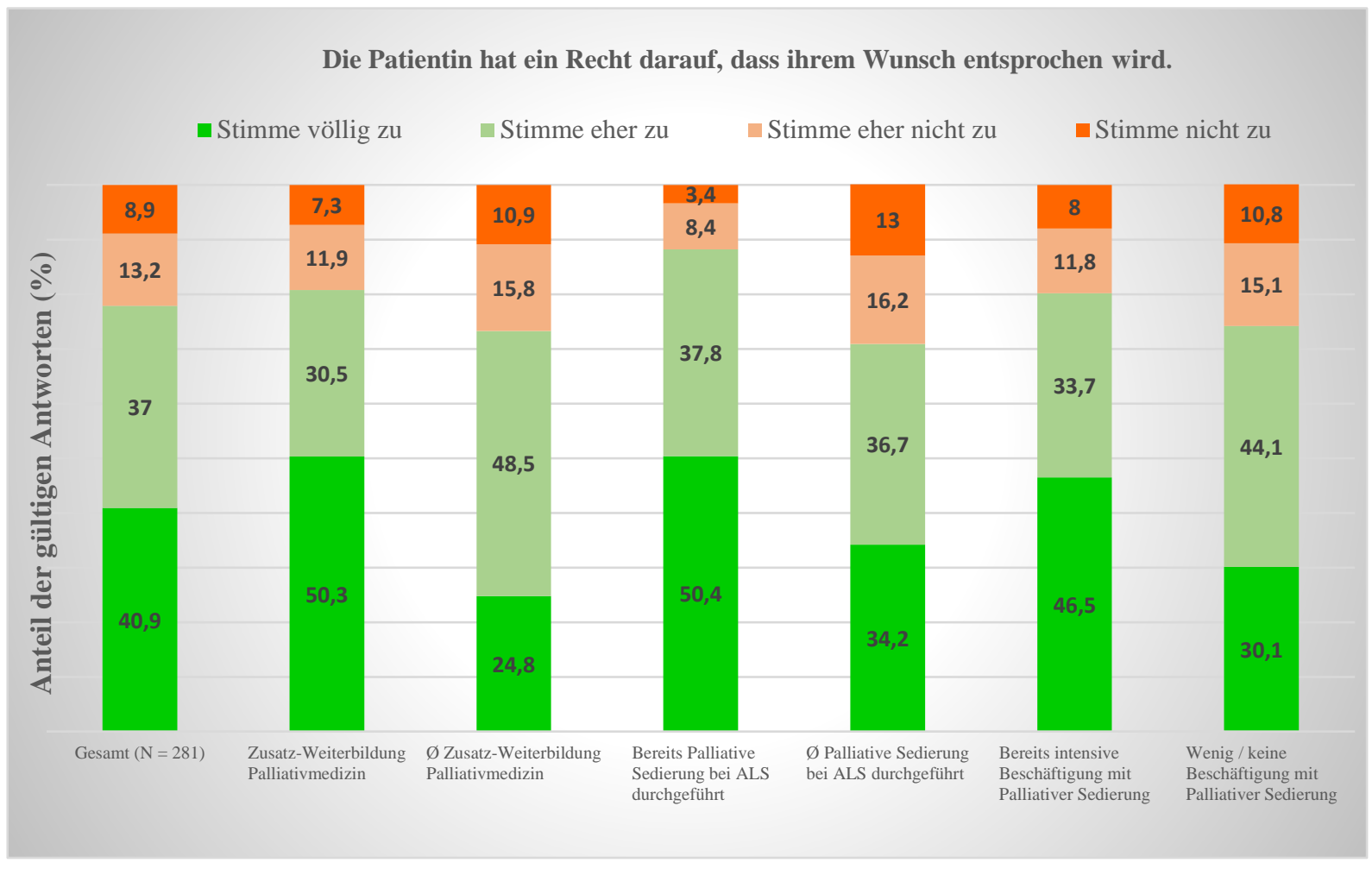

Abbildung 25: Fallbeispiel 2.2, Auswertung Item 2

Insgesamt vertraten $78 \%$ der Antwortenden die Meinung, dass die Patientin ein Recht auf die Durchführung der gewünschten Maßnahmen habe. Es zeigten sich signifikante Gruppenunterschiede zwischen den Befragten mit und ohne Zusatzweiterbildung Palliativmedizin ( $\mathrm{p}=0,00029)$ und zwischen den Befragten mit und ohne Erfahrung mit Palliativer Sedierung bei ALS ( $\mathrm{p}=0,00033)$.

Innerhalb der Gruppe mit Zusatz-Weiterbildung Palliativmedizin wurde das Item deutlich zustimmender bewertet als in der Vergleichsgruppe ohne Zusatz-Weiterbildung. Wie in Abbildung 25 dargestellt, zeigte sich dieser Unterschied insbesondere hinsichtlich der Antwortmöglichkeit „Stimme völlig zu“. Auch antworteten die ÄrztInnen mit Erfahrung mit Palliativer Sedierung bei ALS zustimmender als die entsprechende Vergleichsgruppe. Hinsichtlich der vorangegangenen Beschäftigung mit Palliativer Sedierung ergaben sich keine statistisch signifikanten Gruppenunterschiede. 
Item 3: Ich würde dem Wunsch nur nachkommen, wenn dieser in einer Patientenverfügung dokumentiert ist.

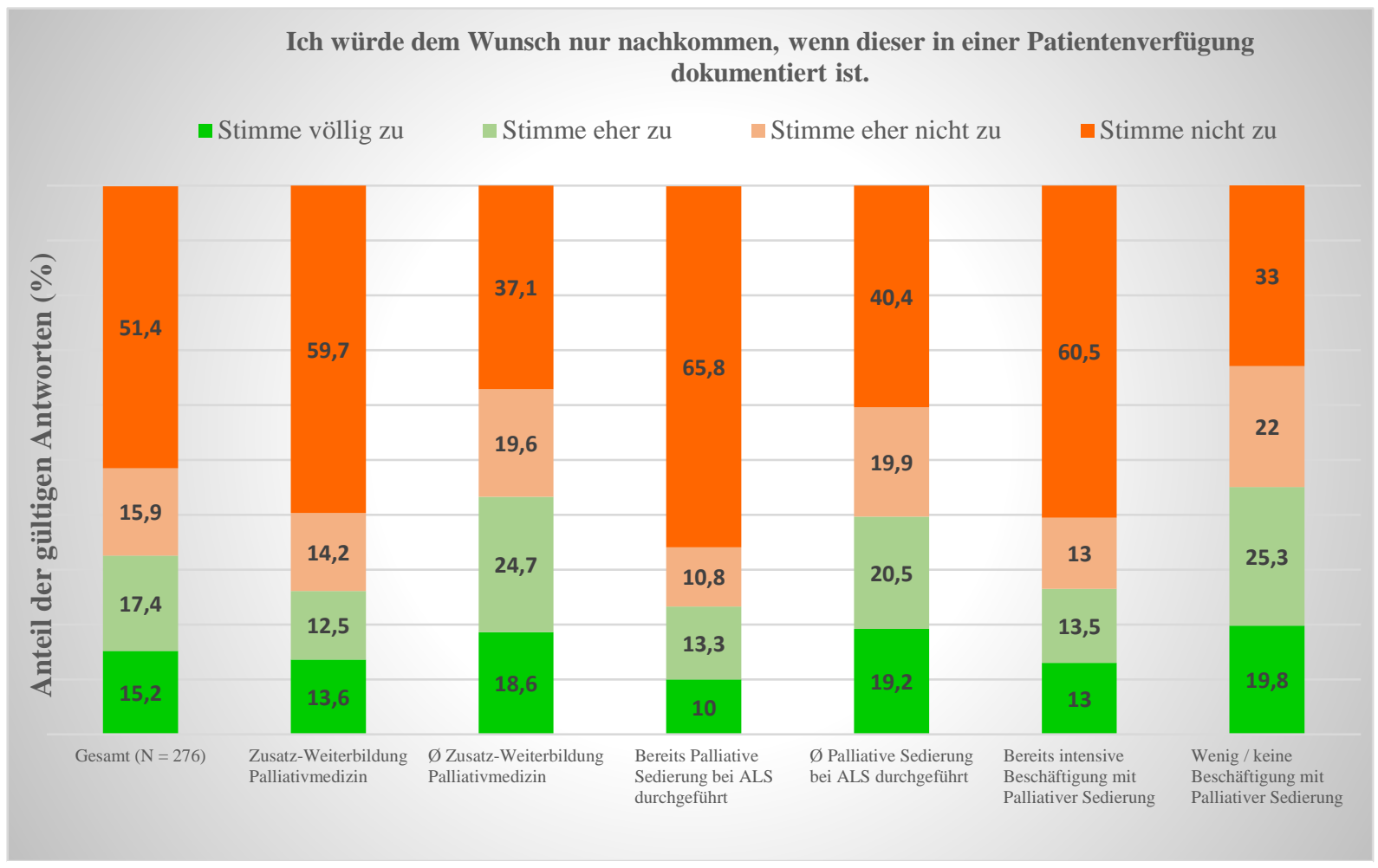

Abbildung 26: Fallbeispiel 2.2, Auswertung Item 3

Insgesamt $67 \%$ der Antwortenden lehnten es ab, dem Wunsch der Patientin nur nachzukommen, wenn dieser in einer Patientenverfügung dokumentiert ist. Es sei darauf hingewiesen, dass dieses Item von 20 der insgesamt 296 Antwortenden nicht bewertet wurde. Signifikante Gruppenunterschiede zeigten sich sowohl zwischen den Befragten mit und ohne Zusatz-Weiterbildung Palliativmedizin $(\mathrm{p}=0,00084)$ als auch zwischen den Befragten mit und ohne Erfahrung mit Palliativer Sedierung bei ALS ( $p=0,00008)$ und zwischen den Befragten mit und ohne vorherige intensive Beschäftigung mit der Thematik $(\mathrm{p}=0,00008)$.

Wie in Abbildung 26 zu sehen ist, reagierten die TeilnehmerInnen mit Zusatz-Weiterbildung Palliativmedizin weniger zustimmend als die TeilnehmerInnen ohne Zusatz-Weiterbildung. Zudem antworteten innerhalb der Gruppe „Bereits Palliative Sedierung bei ALS durchgeführt“ knapp $77 \%$ nicht oder eher nicht zustimmend, in der Vergleichsgruppe waren es $60 \%$. Außerdem wurde das Item von den Teilnehmenden mit intensiver vorangegangener Beschäftigung mit der Thematik weniger zustimmend bewertet als von den Antwortenden mit wenig oder fehlender Vorkenntnis. 


\section{Item 4: Ich würde dem Wunsch nur nachkommen, wenn sich die Patientin bereits unmittelbar in der Sterbephase befindet.}

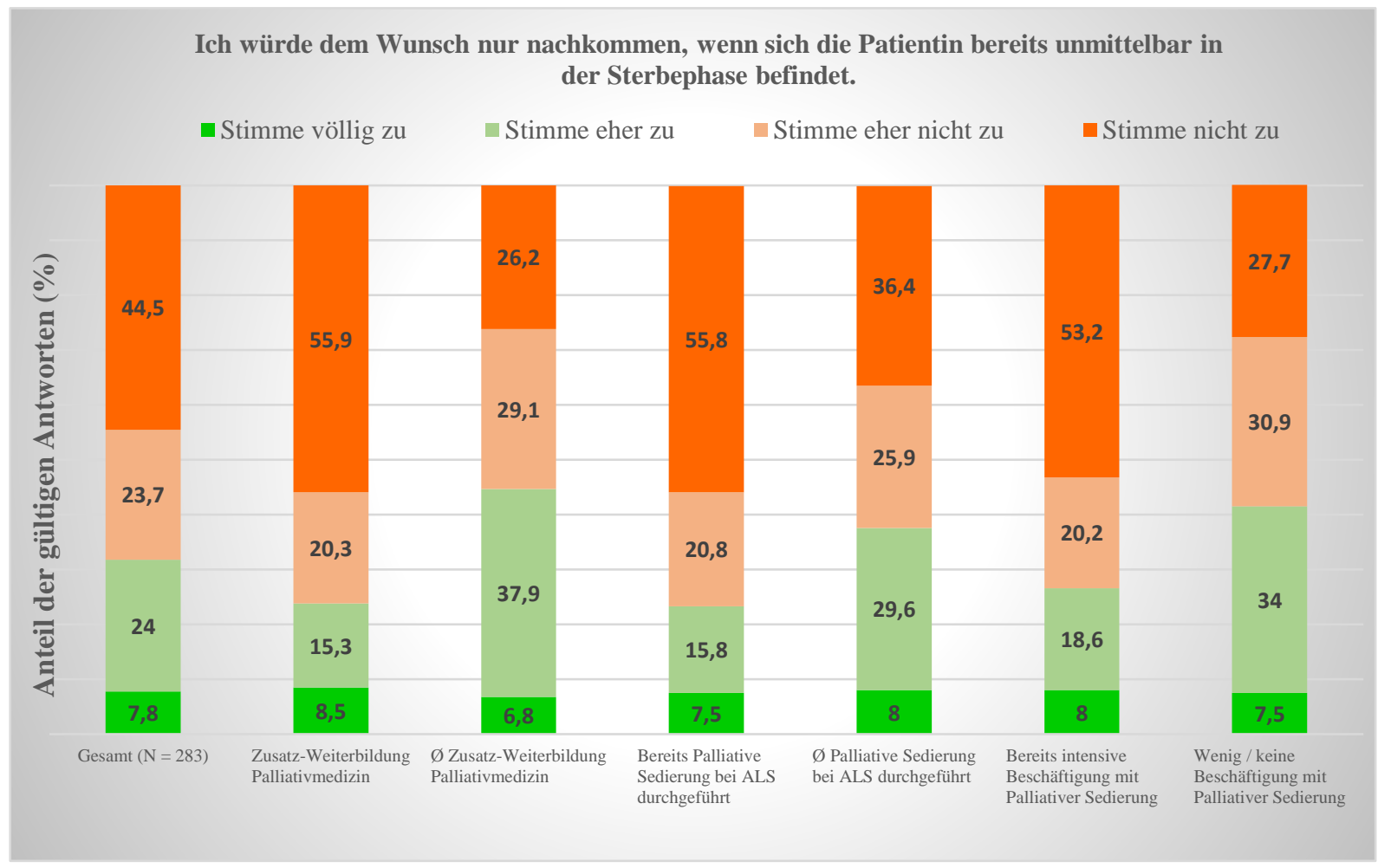

Abbildung 27: Fallbeispiel 2.2, Auswertung Item 4

Insgesamt $68 \%$ der Antwortenden lehnten es ab, dem Wunsch der Patientin nur nachzukommen, wenn diese sich bereits unmittelbar in der Sterbephase befindet. Es zeigten sich signifikante Gruppenunterschiede zwischen den Teilnehmenden mit und ohne ZusatzWeiterbildung Palliativmedizin $(\mathrm{p}=0,00001)$ und zwischen den Teilnehmenden mit und ohne vorherige intensive Beschäftigung mit der Thematik ( $\mathrm{p}=0,00039)$.

Die Teilnehmenden mit Zusatz-Weiterbildung Palliativmedizin reagierten auf die beschriebene Beschränkung auf die unmittelbare Sterbephase häufiger ablehnend als die Antwortenden ohne Zusatz-Weiterbildung. Auch antworteten die ÄrztInnen mit intensiver vorangegangener Beschäftigung mit Palliativer Sedierung weniger zustimmend als die Vergleichsgruppe. Die detaillierten Ergebnisse können der Abbildung 27 entnommen werden. Hinsichtlich der unabhängigen Variable „Erfahrung mit Palliativer Sedierung von ALS-PatientInnen“"konnte kein statistisch signifikanter Unterschied festgestellt werden. 


\section{Item 5: Der Verzicht auf künstliche Ernährung bei kontinuierlicher tiefer Sedierung wäre in meinen Augen mit einer Tötung auf Verlangen gleichzusetzen.}

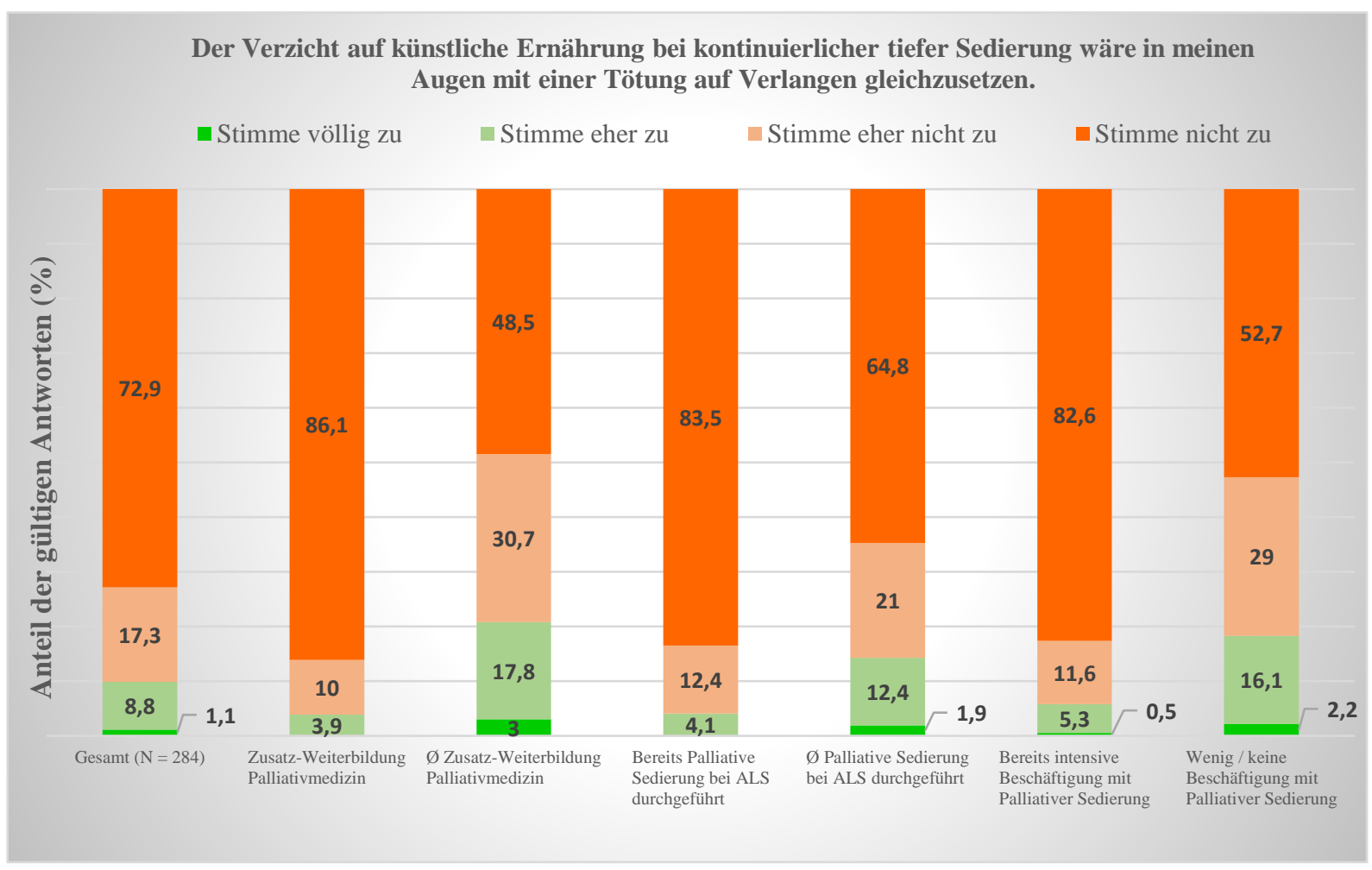

Abbildung 28: Fallbeispiel 2.2, Auswertung Item 5

Eine Gleichsetzung der gewünschten Maßnahmen mit einer Tötung auf Verlangen lehnten insgesamt $90 \%$ der Antwortenden ab. Es zeigten sich signifikante Gruppenunterschiede sowohl zwischen den Befragten mit und ohne Zusatz-Weiterbildung Palliativmedizin $(\mathrm{p}<0,00001)$ als auch zwischen den Befragten mit und ohne Erfahrung mit Palliativer Sedierung bei ALS ( $\mathrm{p}=0,0003)$ und zwischen den Befragten mit und ohne vorherige intensive Beschäftigung mit der Thematik $(\mathrm{p}<0,00001)$.

Wie in Abbildung 28 zu sehen ist, wurde das Item innerhalb der Gruppe mit Zusatz-Weiterbildung Palliativmedizin häufiger ablehnend beurteilt als in der Gruppe ohne ZusatzWeiterbildung. Auch reagierte die Gruppe „Bereits Palliative Sedierung bei ALS durchgeführt“" weniger zustimmend als die Vergleichsgruppe. Außerdem war die Zustimmung unter den Antwortenden mit intensiver vorangegangener Auseinandersetzung mit der Thematik niedriger als unter den Teilnehmenden mit wenig oder fehlenden Vorkenntnissen. 
Item 6: Der Verzicht auf künstliche Ernährung bei kontinuierlicher und tiefer Sedierung führt zu einem früheren Versterben als es dem Erkrankungsverlauf ohne diese Maßnahme entsprechen würde.

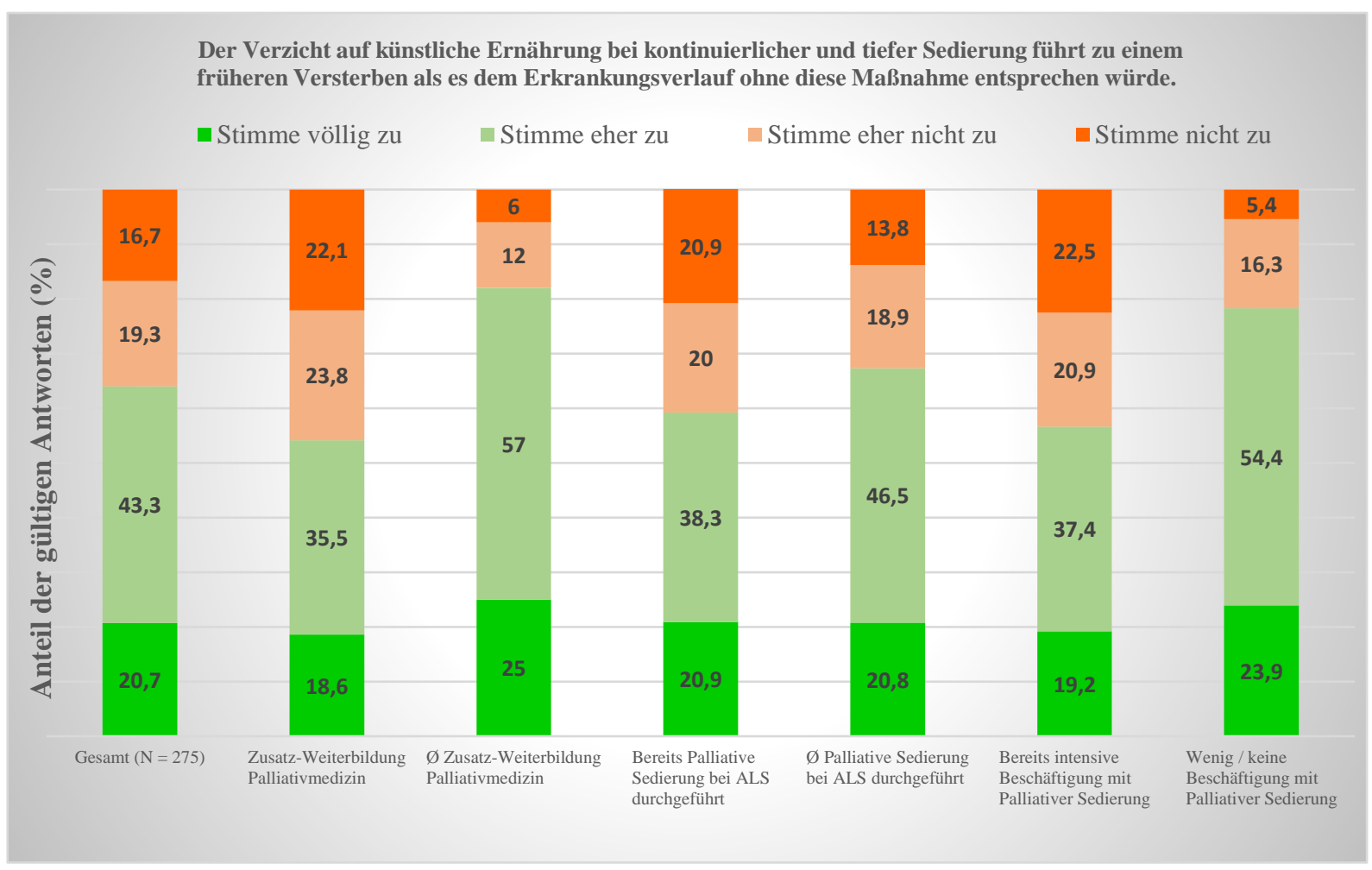

Abbildung 29: Fallbeispiel 2.2, Auswertung Item 6

Gruppenübergreifend waren $64 \%$ der teilnehmenden ÄrztInnen der Ansicht, dass der Verzicht auf künstliche Ernährung bei gleichzeitiger tiefer und kontinuierlicher Sedierung zu einem vergleichsweise früheren Versterben führe. Es sei darauf hingewiesen, dass dieses Item von 21 der insgesamt 296 Antwortenden nicht bewertet wurde. Signifikante Gruppenunterschiede zeigten sich zwischen den Teilnehmenden mit und ohne Zusatz-Weiterbildung Palliativmedizin $(\mathrm{p}=0,00007)$ und zwischen den Teilnehmenden mit und ohne vorherige intensive Beschäftigung mit der Thematik ( $p=0,00123)$.

Etwa $54 \%$ der Antwortenden mit Zusatz-Weiterbildung Palliativmedizin stimmten dem Item völlig oder eher zu (siehe Abbildung 29). In der Gruppe ohne Zusatz-Weiterbildung waren es hingegen $82 \%$. Auch reagierten die TeilnehmerInnen mit intensiver vorangegangener Beschäftigung mit der Thematik weniger zustimmend als die Vergleichsgruppe. Hinsichtlich der praktischen Erfahrung mit der Palliativen Sedierung von ALS-PatientInnen ergaben sich keine statistisch signifikanten Gruppenunterschiede. 


\section{Item 7: Eine künstliche Ernährung bei einer kontinuierlichen tiefen Sedierung wäre in meinen Augen medizinisch nicht indiziert.}

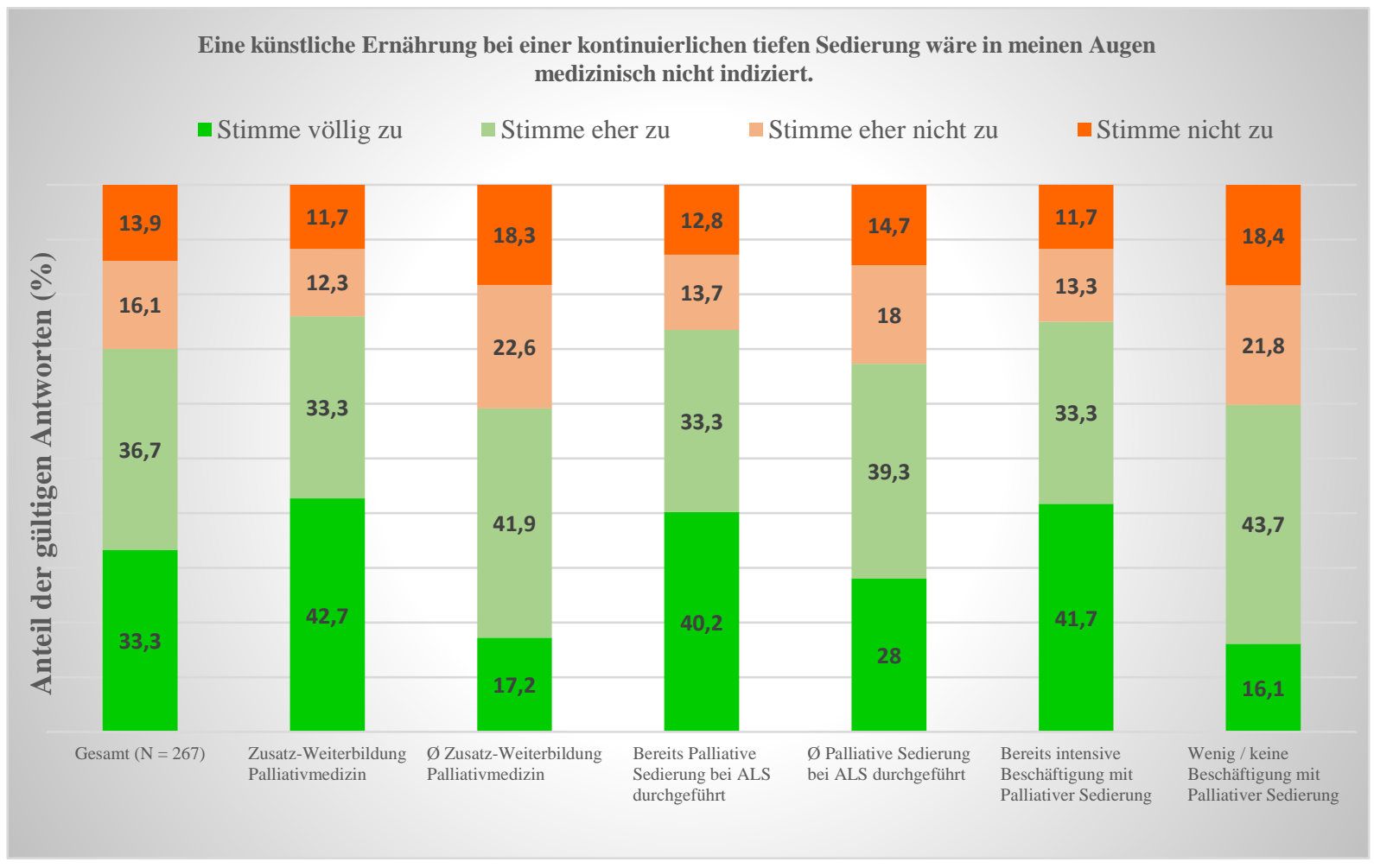

Abbildung 30: Fallbeispiel 2.2, Auswertung Item 7

Insgesamt vertraten $70 \%$ der Teilnehmenden die Meinung, dass eine künstliche Ernährung bei einer kontinuierlichen tiefen Sedierung medizinisch nicht indiziert sei. Dieses Item wurde jedoch von 29 der insgesamt 296 Antwortenden nicht bewertet. Es zeigten sich signifikante Gruppenunterschiede zwischen den Befragten mit und ohne ZusatzWeiterbildung Palliativmedizin ( $\mathrm{p}=0,00005)$ und zwischen den Befragten mit und ohne vorherige intensive Beschäftigung mit der Thematik $(\mathrm{p}=0,0001)$.

Unter den Antwortenden mit Zusatz-Weiterbildung Palliativmedizin antworteten $76 \%$ völlig oder eher zustimmend (siehe Abbildung 30). In der Gruppe ohne Zusatz-Weiterbildung waren es hingegen $59 \%$. Unter den TeilnehmerInnen, die sich bereits intensiv mit Palliativer Sedierung auseinander gesetzt hatten, war die Zustimmung zudem deutlich höher als in der Vergleichsgruppe. Hinsichtlich der unabhängigen Variable „Bereits Palliative Sedierung bei ALS durchgeführt" bestanden keine statistisch signifikanten Gruppenunterschiede. 
Zusatzfrage: Welches Vorgehen würden Sie im Kontext der zuletzt beschriebenen Situation als moralisch belastender empfinden?

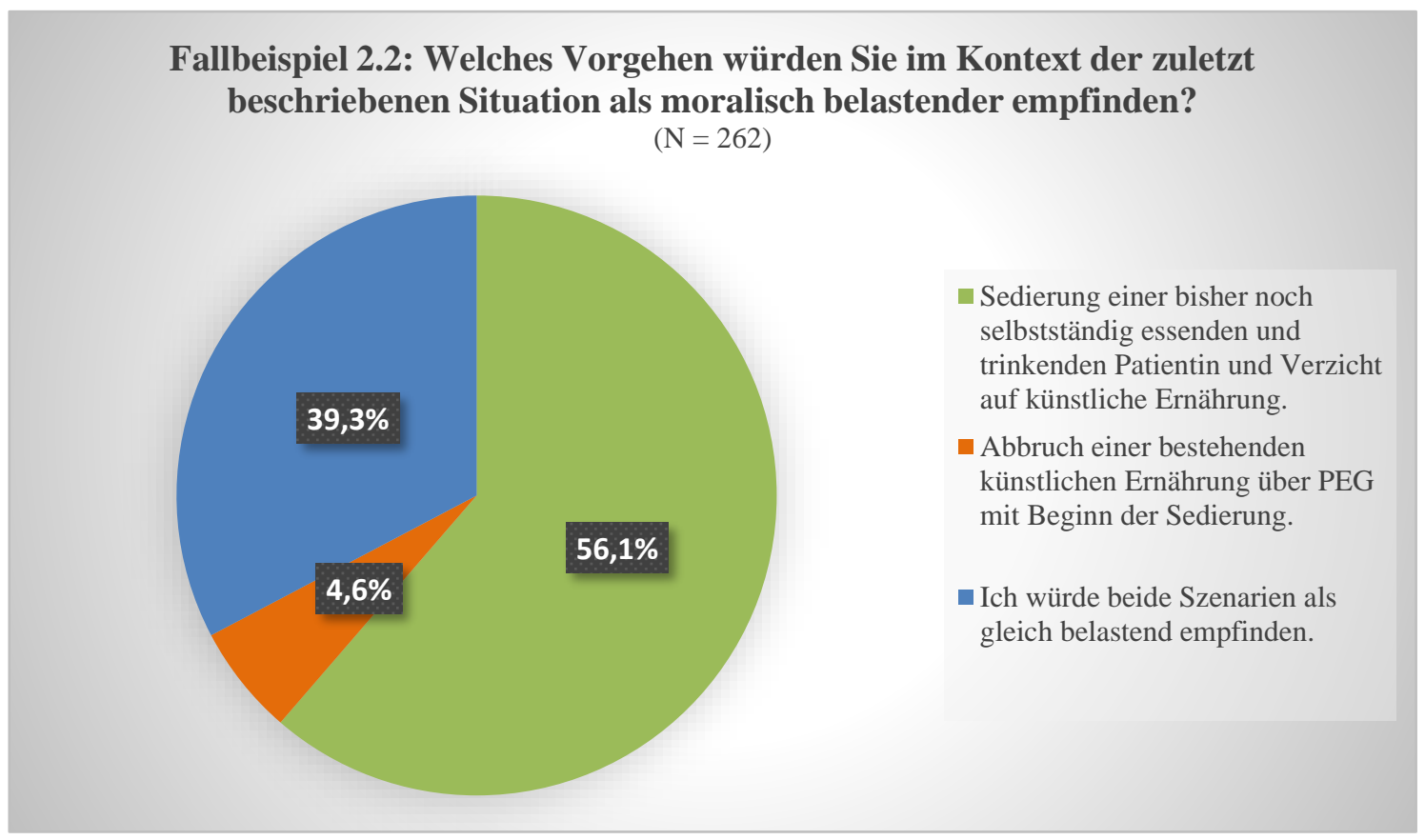

Abbildung 31: Fallbeispiel 2.2, Auswertung Zusatzfrage

Zum Abschluss des Fallbeispiels 2.2 wurde dieselbe Zusatzfrage gestellt wie zum Abschluss des Fallbeispiels 1.2. Es ist auffällig, dass 34 der insgesamt 296 Antwortenden dieses Item nicht bewertet haben.

Wiederum stufte über die Hälfte der Antwortenden die Sedierung einer bisher noch selbstständig essenden und trinkenden Patientin bei gleichzeitigem Verzicht auf künstliche Ernährung als moralisch belastender ein als den Abbruch einer bereits bestehenden künstlichen Ernährung mit Beginn der Sedierung. Knapp $40 \%$ der Teilnehmenden bewerteten beide Vorgehensweisen als gleich belastend (siehe Abbildung 31).

Es bestanden keine statistisch signifikanten Unterschiede für die unabhängigen Variablen „Vorherige Beschäftigung mit dem Begriff der Palliativen Sedierung“, „Zusatz-Weiterbildung Palliativmedizin“ und „Erfahrung mit Palliativer Sedierung von ALS-PatientInnen“. 


\subsection{Fallbeispiel 3: Elektive Beatmungsbeendigung unter Palliativer Sedierung}

Im Fallbeispiel 3 wird ein ALS-Patient im Endstadium der Erkrankung beschrieben, der aufgrund fortschreitender respiratorischer Insuffizienz invasiv beatmet wird. Der Patient ist durch seine körperlichen Symptome stark belastet und wünscht nun eine Beendigung der invasiven Beatmung bei gleichzeitiger Palliativer Sedierung. Den Teilnehmenden wurden wiederum fünf Items präsentiert, die mithilfe einer vierstufigen Skala (,Stimme völlig zu“ bis „Stimme nicht zu“) bewertet werden sollten.

\section{Item 1: Der Wunsch des Patienten ist für mich nachvollziehbar.}

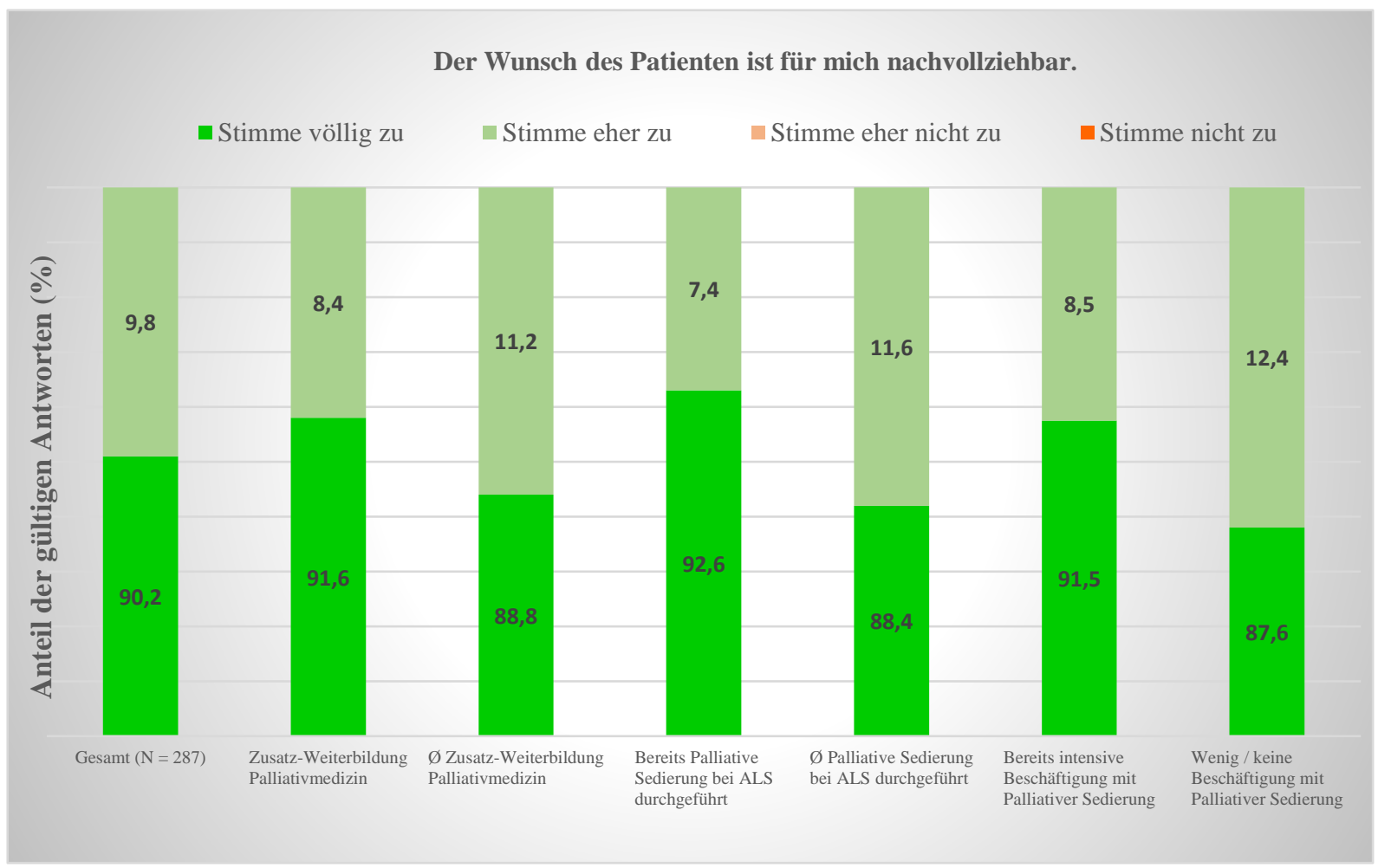

Abbildung 32: Fallbeispiel 3, Auswertung Item 1

Alle teilnehmenden ÄrztInnen gaben an, den Wunsch des Patienten nachvollziehen zu können, darunter antworteten 90 \% mit „Stimme völlig zu“. Die detaillierten Ergebnisse aller Untergruppen können der Abbildung 32 entnommen werden. Es bestanden keine statistisch signifikanten Gruppenunterschiede hinsichtlich der drei unabhängigen Variablen. 


\section{Item 2: Der Patient hat ein Recht darauf, dass seinem Wunsch entsprochen wird.}

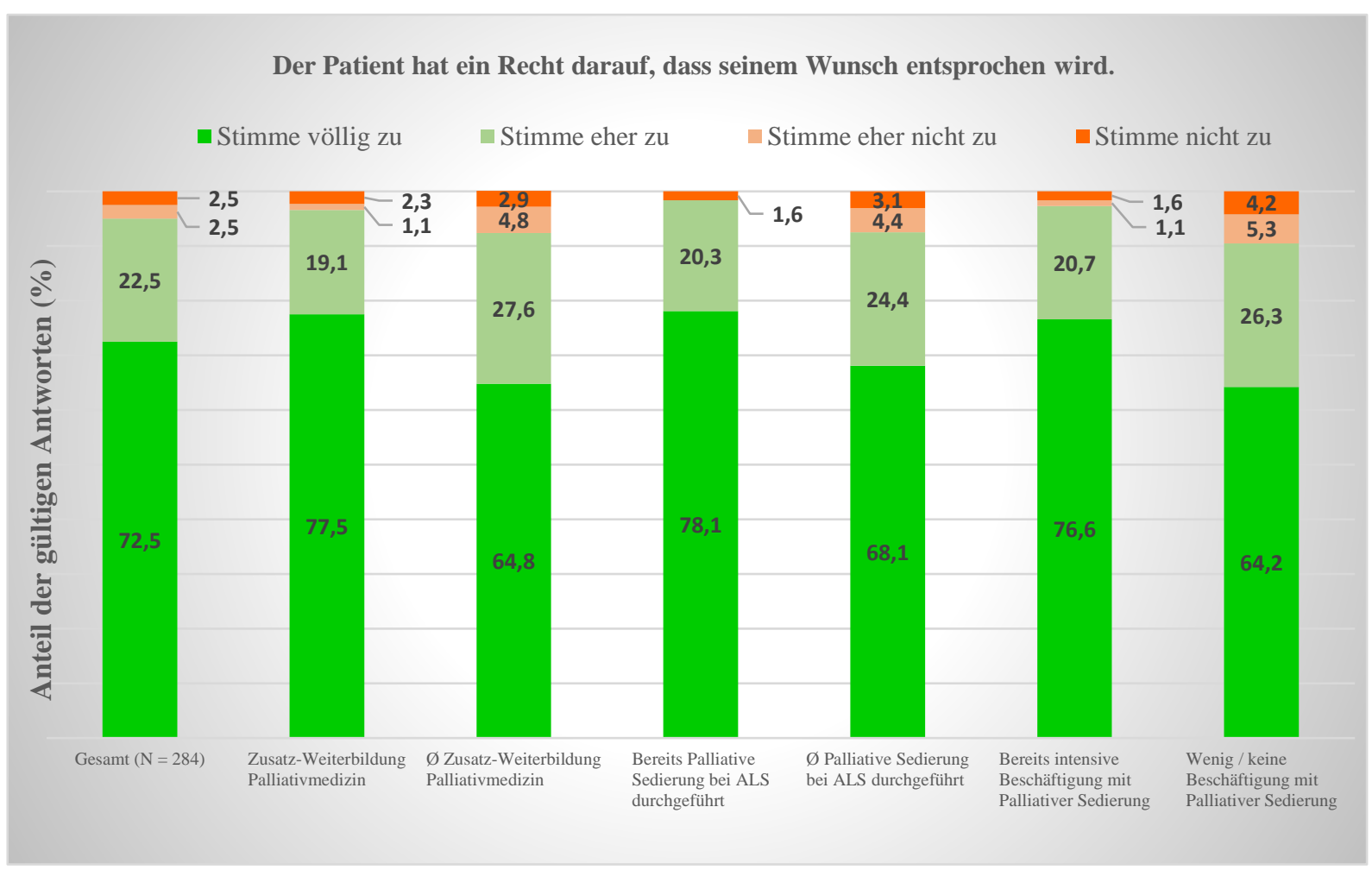

Abbildung 33: Fallbeispiel 3, Auswertung Item 2

Insgesamt $95 \%$ der Antwortenden waren der Ansicht, dass der Patient ein Recht darauf habe, dass seinem Wunsch entsprochen wird. Wiederum konnten keine statistisch signifikanten Unterschiede zwischen den in Abbildung 33 dargestellten Untergruppen festgestellt werden. 
Item 3: Ich würde dem Wunsch nur nachkommen, wenn dieser in einer Patientenverfügung dokumentiert ist.

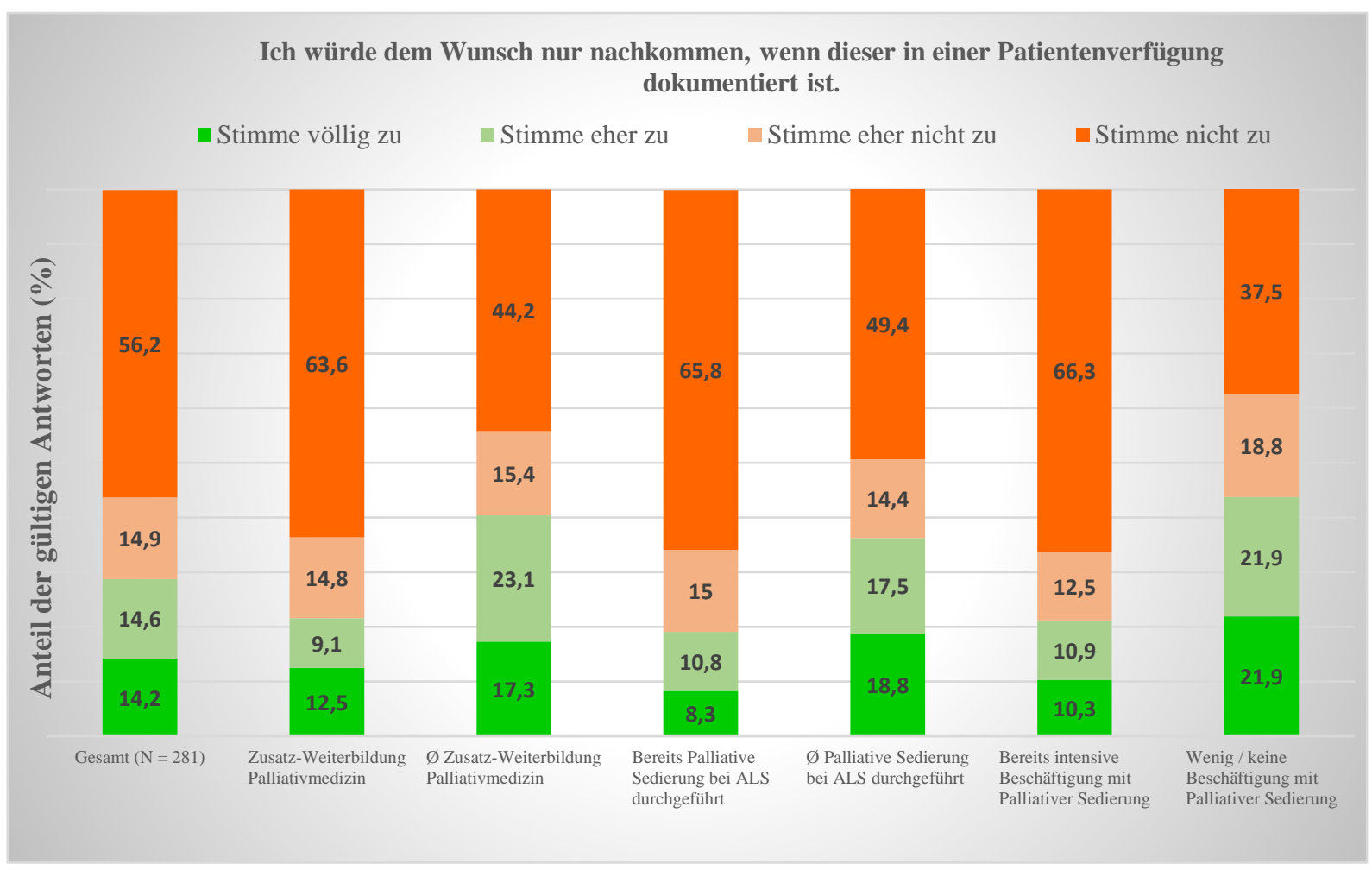

Abbildung 34: Fallbeispiel 3, Auswertung Item 3

Etwa $70 \%$ der TeilnehmerInnen lehnten es ab, dem Wunsch des Patienten nach Beatmungsbeendigung bei gleichzeitiger Palliativer Sedierung nur nachzukommen, wenn dieser in einer Patientenverfügung festgehalten ist. Es zeigten sich signifikante Gruppenunterschiede zwischen den Befragten mit und ohne Zusatz-Weiterbildung Palliativmedizin $(\mathrm{p}=0,00129)$ und zwischen den Befragten mit und ohne vorherige intensive Beschäftigung mit der Thematik $(\mathrm{p}<0,00001)$.

Unter den Antwortenden mit Zusatz-Weiterbildung Palliativmedizin antworteten $78 \%$ nicht oder eher nicht zustimmend (siehe Abbildung 34). Unter den Teilnehmenden ohne Zusatz-Weiterbildung waren es hingegen $60 \%$. Außerdem wurde das Item unter den ÄrztInnen mit intensiver vorangegangener Beschäftigung mit Palliativer Sedierung ablehnender bewertet als in der Vergleichsgruppe. Bezüglich der unabhängigen Variable „Erfahrung mit Palliativer Sedierung von ALS-PatientInnen“ ergaben sich keine statistisch signifikanten Gruppenunterschiede. 


\section{Item 4: Ich würde dem Wunsch nur nachkommen, wenn sich der Patient bereits unmittelbar in der Sterbephase befindet.}

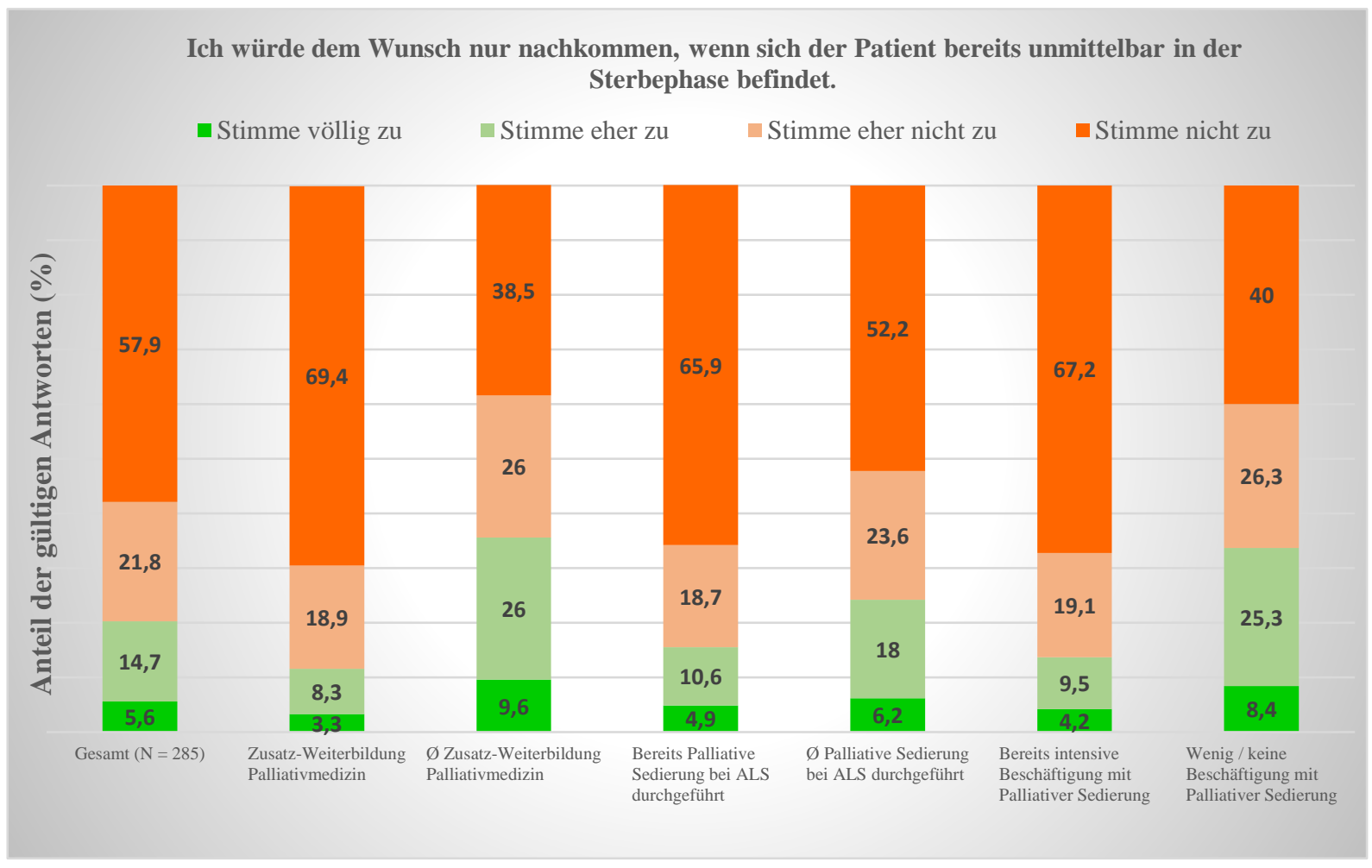

Abbildung 35: Fallbeispiel 3, Auswertung Item 4

Etwa $80 \%$ der Teilnehmenden lehnten es ab, dem Wunsch erst nachzukommen, wenn sich der Patient bereits unmittelbar in der Sterbephase befindet. Es zeigten sich signifikante Gruppenunterschiede zwischen den Teilnehmenden mit und ohne Zusatz-Weiterbildung Palliativmedizin $(\mathrm{p}<0,00001)$ und zwischen den Teilnehmenden mit und ohne vorherige intensive Beschäftigung mit der Thematik ( $p<0,00001)$.

Die Antwortenden mit Zusatz-Weiterbildung Palliativmedizin wählten zu $88 \%$ eine ablehnende Antwortmöglichkeit, unter den Antwortenden ohne Zusatz-Weiterbildung waren es hingegen $66 \%$ (siehe Abbildung 35). Etwa $86 \%$ der Teilnehmenden mit intensiver vorangegangener Beschäftigung mit Palliativer Sedierung antworteten nicht oder eher nicht zustimmend. In der Vergleichsgruppe mit wenig oder fehlenden Vorkenntnissen waren es $66 \%$. Es bestanden keine statistisch signifikanten Unterschiede hinsichtlich der Variable „Erfahrung mit Palliativer Sedierung von ALS-PatientInnen“. 
Item 5: Die Beendigung der invasiven Beatmung unter Sedierung wäre in meinen Augen mit einer Tötung auf Verlangen gleichzusetzen.

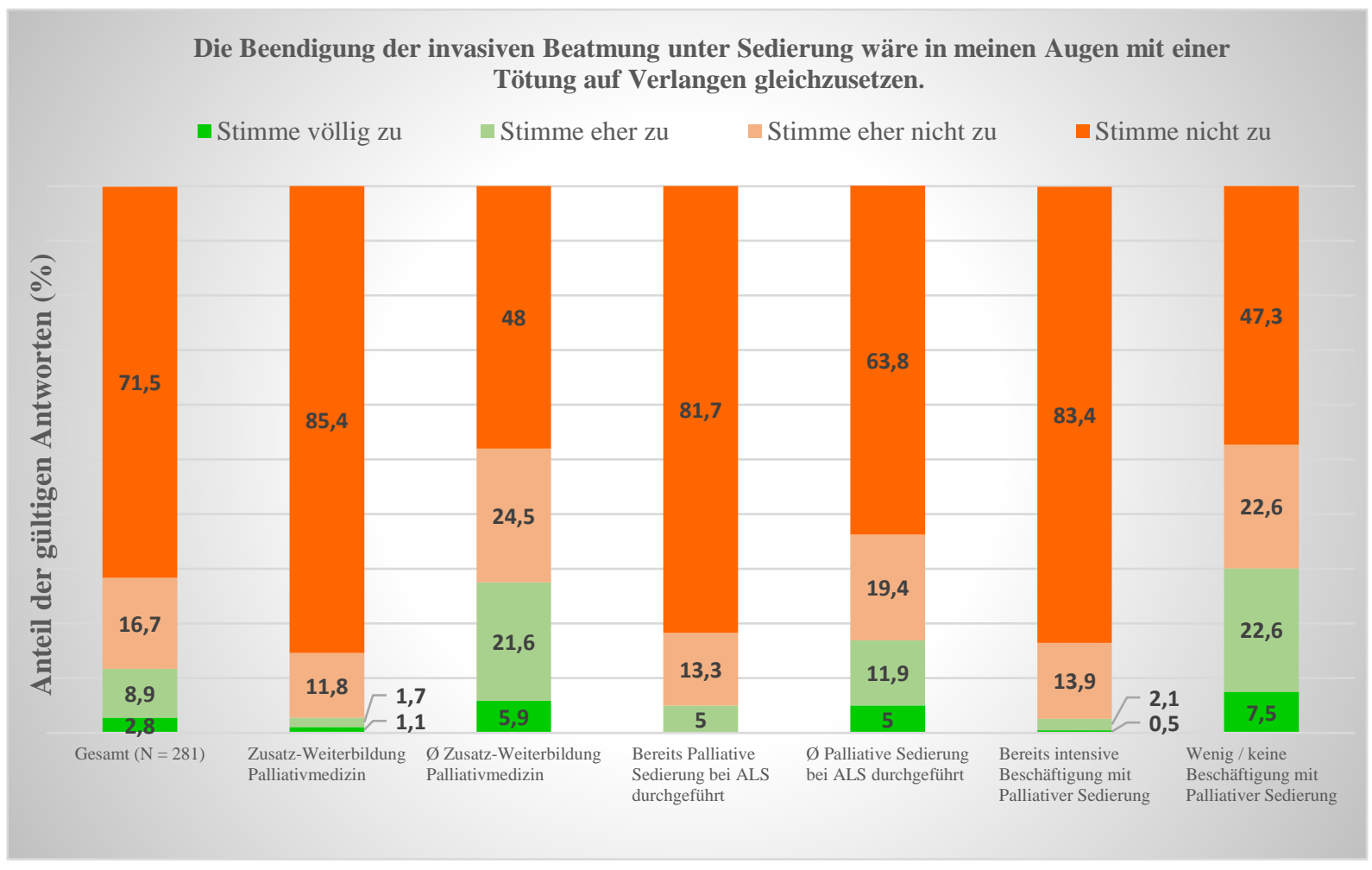

Abbildung 36: Fallbeispiel 3, Auswertung Item 5

Schließlich wurde die Frage adressiert, ob die Beendigung der invasiven Beatmung mit einer Tötung auf Verlangen gleichzusetzen sei. Etwa 88 \% der teilnehmenden ÄrztInnen reagierten ablehnend auf eine solche Gleichsetzung (siehe Abbildung 36). Es zeigten sich signifikante Gruppenunterschiede sowohl zwischen den Befragten mit und ohne ZusatzWeiterbildung Palliativmedizin ( $<<0,00001)$ als auch zwischen den Befragten mit und ohne Erfahrung mit der Palliativen Sedierung bei ALS ( $p=0,00045)$ und zwischen den Befragten mit und ohne vorherige intensive Beschäftigung mit der Thematik ( $\mathrm{p}<$ $0,00001)$.

Die TeilnehmerInnen mit Zusatz-Weiterbildung Palliativmedizin bewerteten das Item mit etwa $97 \%$ häufiger ablehnend als die Antwortenden ohne Zusatz-Weiterbildung. Zudem antwortete die Gruppe „Bereits Palliative Sedierung bei ALS durchgeführt“ mit $95 \%$ häufiger ablehnend als die Vergleichsgruppe ohne diese praktische Erfahrung. Auch ging eine intensivere Beschäftigung mit einer signifikant stärkeren Ablehnung des Items einher. 


\subsection{Gegenüberstellung der Fallbeispiele}

Im Folgenden werden die fünf verschiedenen Szenarien $(1.1,1.2,2.1,2.2,3)$ miteinander verglichen. Durch diesen Vergleich soll geklärt werden, ob Veränderungen der Kontextvariablen (körperliche oder psychische Symptomatik, Verzicht auf künstliche Ernährung) zu einer Veränderung der Zustimmung zur Palliativen Sedierung führen. Verglichen werden dabei die folgenden Items, die im Rahmen aller fünf Szenarien von den Teilnehmenden bewertet wurden:

Tabelle 6: Items Szenarienvergleich

Der Wunsch der Patientin/des Patienten ist für mich nachvollziehbar.

Die Patientin/der Patient hat ein Recht darauf, dass ihrem/seinem Wunsch entsprochen wird.

Ich würde dem Wunsch nur nachkommen, wenn sich die Patientin/der Patient bereits unmittelbar in der Sterbephase befindet.

Zusätzlich zu diesem Vergleich der fünf Szenarien werden wiederum signifikante Unterschiede zwischen Antwortenden mit und ohne Zusatz-Weiterbildung hervorgehoben.

Item 1: Der Wunsch der Patientin/des Patienten ist für mich nachvollziehbar.

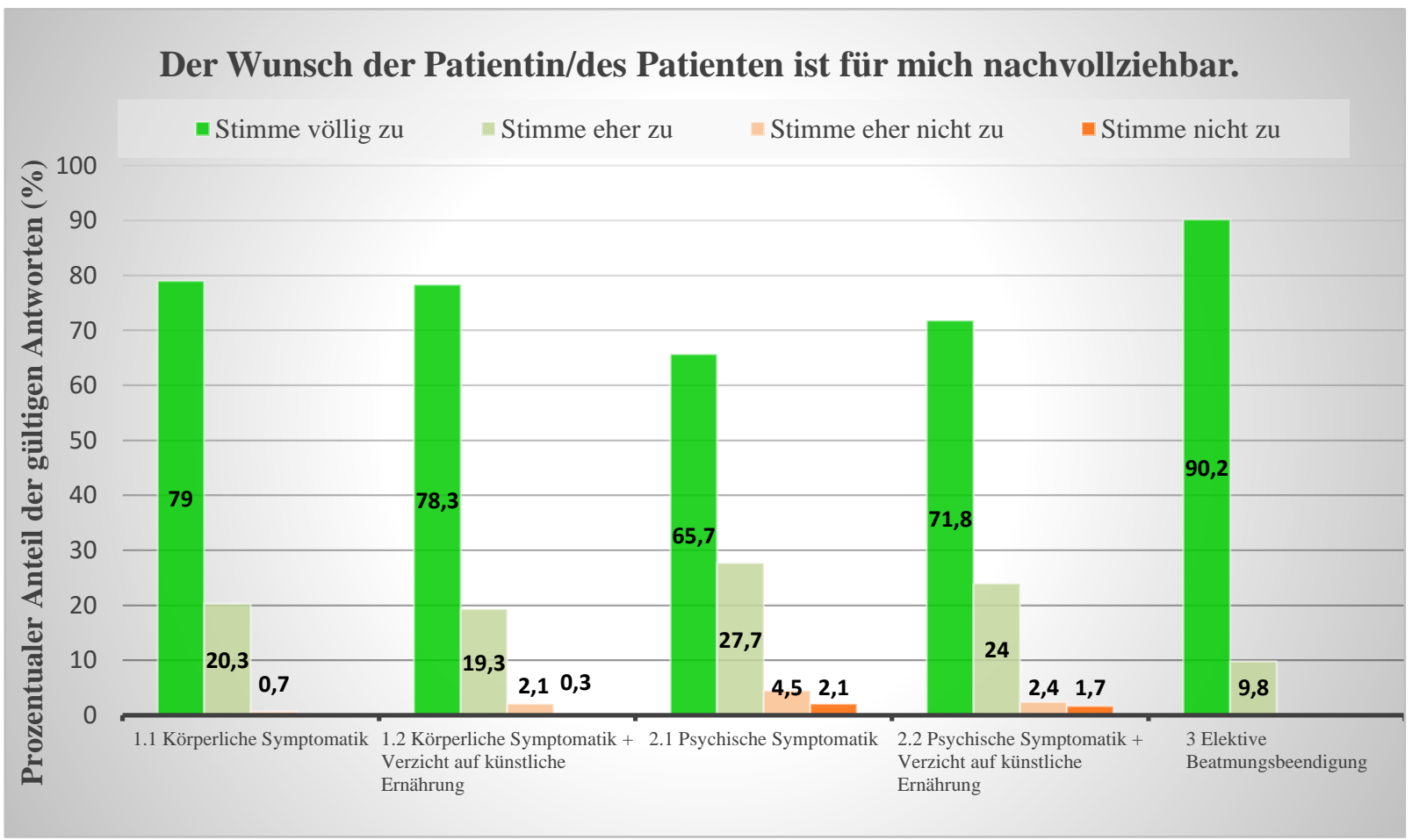

Abbildung 37: Szenarienvergleich Item 1 
Die zustimmenden Antworten überwogen im Rahmen aller fünf Szenarien deutlich. Im Fallbeispiel 1.1 gaben fast $100 \%$ der Antwortenden an, den Wunsch der Patientin nachvollziehen zu können. Auch der in Fallbeispiel 1.2 zusätzlich geäußerte Wunsch nach Verzicht auf künstliche Ernährung führte nicht zu einem signifikanten Abfall der Zustimmung.

Ein ähnliches Bild zeigte sich beim Vergleich der Szenarien 2.1 und 2.2. Auch im Fall der Patientin mit primär psychischer Symptomatik führte der zusätzliche Wunsch nach Verzicht auf künstliche Ernährung nicht zu einem signifikanten Abfall der Zustimmung. Wie in Abbildung $37 \mathrm{zu}$ sehen ist, war die Zustimmung in Szenario 2.2 sogar etwas gröBer, dieser Unterschied ist aber statistisch nicht signifikant.

Bei einem Vergleich der Fallbeispiele 1.1 und 2.1 konnte jedoch ein signifikanter Unterschied $(\mathrm{p}<0,00001)$ festgestellt werden. Das Item wurde im Kontext der Patientin mit körperlicher Symptomatik zustimmender bewertet als im Kontext der Patientin mit psychischer Symptomatik. Beim Vergleich der Fallbeispiele 1.2 und 2.2 zeigten sich keine statistisch signifikanten Zustimmungsunterschiede.

Im Rahmen des Szenarios 3 wurde das Item insgesamt am positivsten bewertet. $100 \%$ der Antwortenden gaben an, den Wunsch des Patienten nach Beatmungsbeendigung bei gleichzeitiger Palliativer Sedierung völlig oder eher nachvollziehen zu können. Damit war die Zustimmung signifikant höher als in den Fallbeispielen 1.1 und 2.1 ( $\mathrm{p}<0,00001)$.

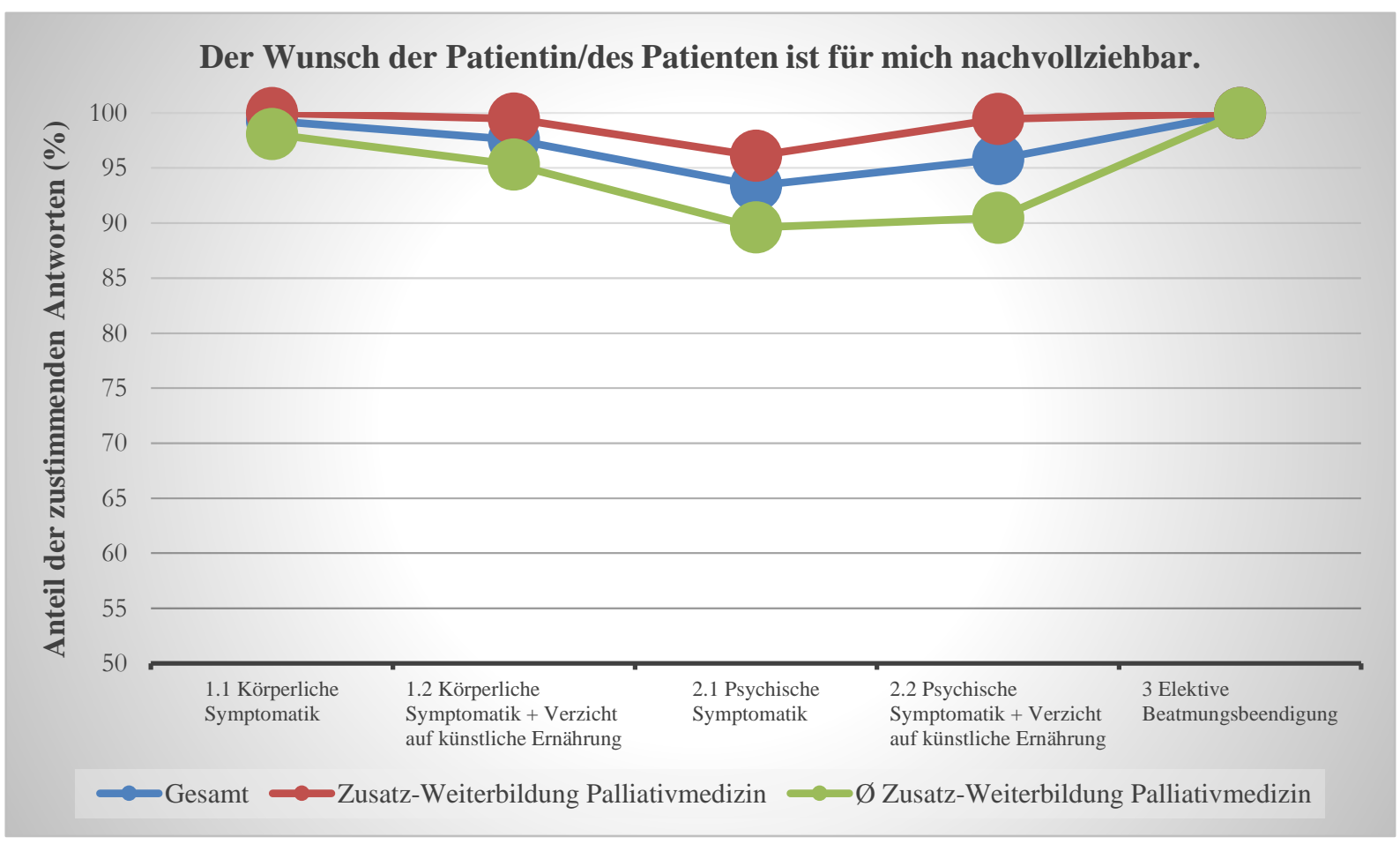

Abbildung 38: Szenarienvergleich mit Differenzierung nach "Zusatz-Weiterbildung Palliativmedizin" Item 1 
In Abbildung 38 wird zusätzlich zum Vergleich der Szenarien auch eine Differenzierung hinsichtlich der Variable „Zusatz-Weiterbildung Palliativmedizin“ vorgenommen. Es ist dabei auf der Ordinatenachse der Anteil der zustimmenden Antworten („Stimme völlig

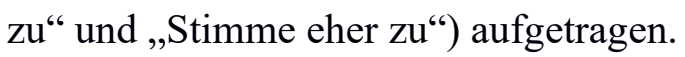

Wie in Abbildung 38 dargestellt, war der Anteil der zustimmenden Antworten in der Gruppe mit Zusatz-Weiterbildung Palliativmedizin szenarienübergreifend etwas höher als in der Gruppe ohne Zusatz-Weiterbildung, mit statistisch signifikanten Unterschieden in den Fallbeispielen 1.2, 2.1 und 2.2.

Bezieht man zusätzlich zum Szenarienvergleich auch die Differenzierung anhand der Variable „Zusatz-Weiterbildung Palliativmedizin“ ein, so zeigt sich beim Vergleich der Fallbeispiele 1.1 und 3 ein Unterschied. Im Rahmen des Fallbeispiels 3 antworteten die Antwortenden ohne Zusatz-Weiterbildung signifikant zustimmender als in Fallbeispiel $1.1(\mathrm{p}=0,00013)$. In der Gruppe mit Zusatz-Weiterbildung bestand hingegen kein signifikanter Zustimmungsunterschied zwischen den beiden Szenarien.

Beim Vergleich der übrigen Szenarien zeigte sich kein Unterschied zwischen den Teilnehmenden mit und ohne Zusatz-Weiterbildung Palliativmedizin.

\section{Item 2: Die Patientin/der Patient hat ein Recht darauf, dass ihrem/seinem Wunsch entsprochen wird.}

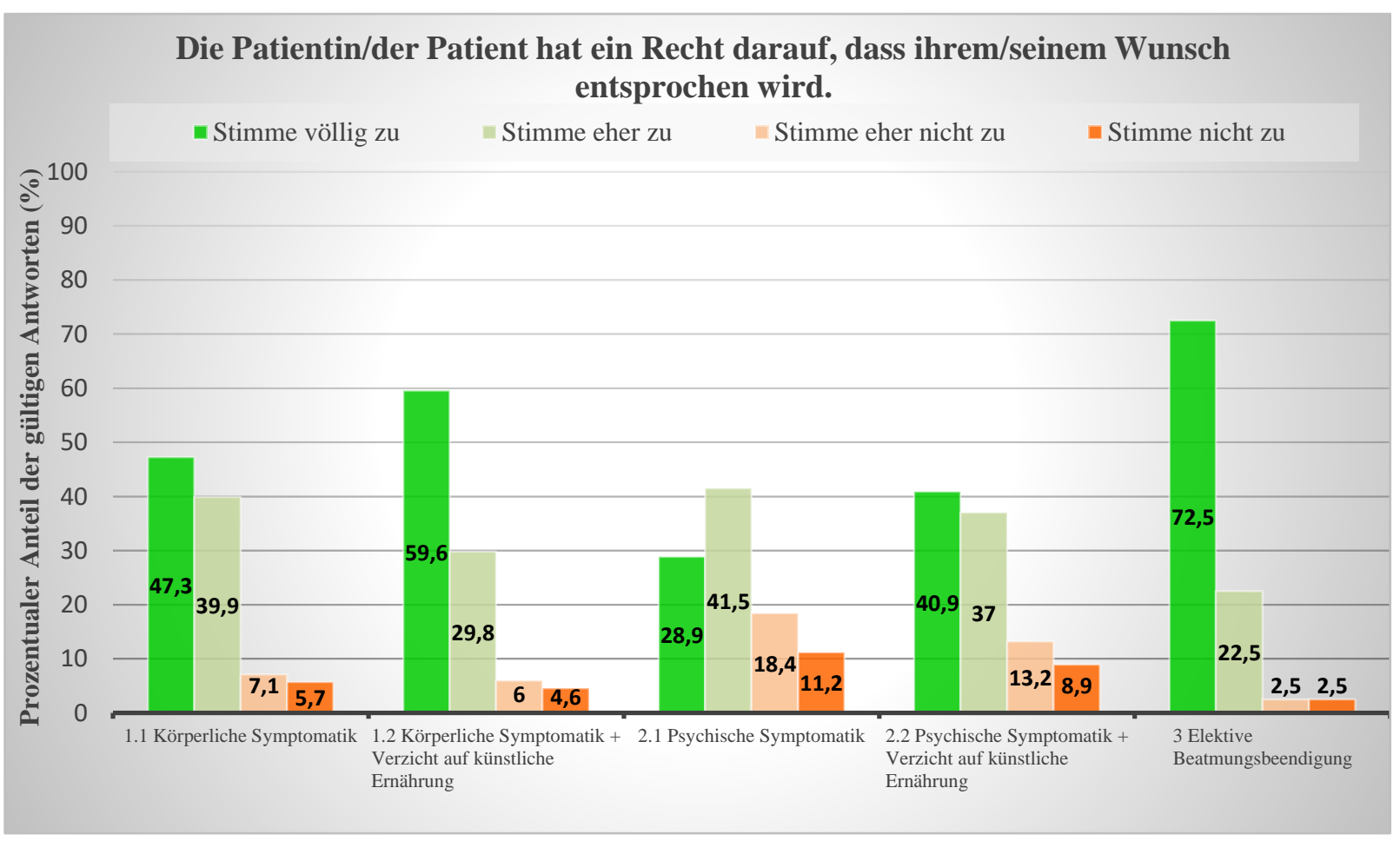


Die Zustimmung zum Item 2 war szenarienübergreifend geringer als die Zustimmung zum Item 1. Im Fallbeispiel 1.1 vertraten etwa $87 \%$ der Antwortenden die Meinung, dass die Patientin ein Recht auf die gewünschten Maßnahmen habe. Der zusätzliche Wunsch nach Verzicht auf künstliche Ernährung führte zu einem signifikanten $(p=0,00006)$ Anstieg der Zustimmung (siehe Abbildung 39).

Ein ähnliches Bild zeigte sich beim Vergleich der Fallbeispiele 2.1 und 2.2. Auch im Fall der Patientin mit psychischer Symptomatik erfolgte ein signifikanter $(p<0,00001)$ Anstieg der Zustimmung, wenn zusätzlich zur kontinuierlichen tiefen Sedierung ein Verzicht auf künstliche Ernährung gewünscht wird.

Beim Vergleich der Fallbeispiele 1.1 und 2.1 wurde wiederum ein signifikantes ( $p<$ 0,00001) Zustimmungsgefälle von körperlicher zu psychischer Symptomatik sichtbar. Dieses Gefälle bestand auch zwischen den Fallbeispielen 1.2 und 2.2. Im Fall der Patientin mit körperlicher Symptomatik vertraten signifikant $(\mathrm{p}<0,00001)$ mehr Teilnehmende die Meinung, dass die Patientin ein Recht auf die gewünschten Maßnahmen habe als im Fall der Patientin mit psychischer Symptomatik.

Außerdem war die Zustimmung im Fall des Patienten mit Wunsch nach elektiver Beatmungsbeendigung signifikant $(\mathrm{p}<0,00001)$ höher als im Fall der Patientin mit einerseits körperlicher und andererseits psychischer Symptomatik.

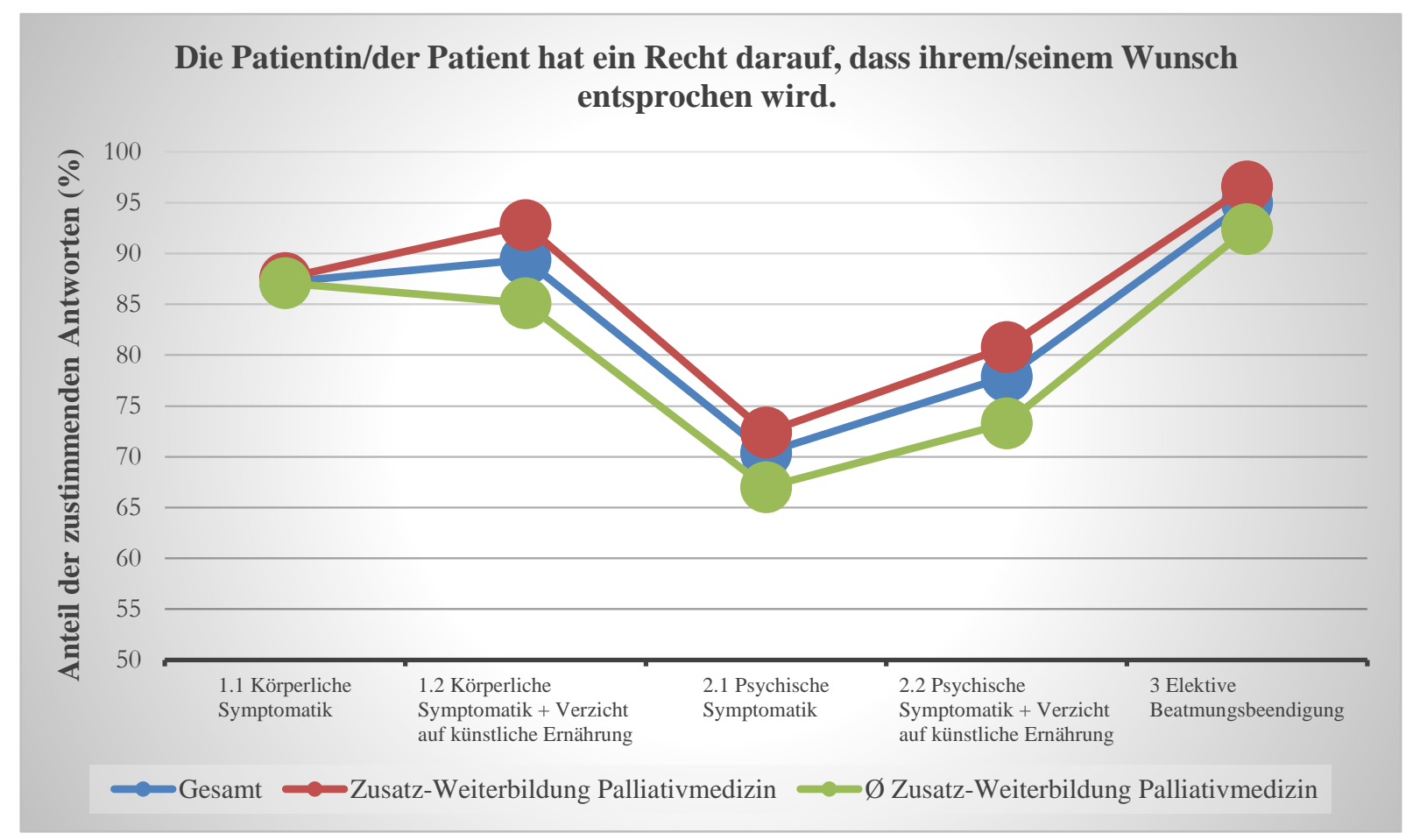


Abbildung 40 enthält wiederum den Vergleich der Szenarien und eine Differenzierung hinsichtlich der Variable „Zusatz-Weiterbildung Palliativmedizin“. Auf der Ordinatenachse ist der Anteil der zustimmenden Antworten (,Stimme völlig zu“ und „Stimme eher $\mathrm{zu}^{6 *}$ ) aufgetragen.

Auch im Kontext dieses Items war die Zustimmung in der Gruppe mit Zusatz-Weiterbildung szenarienübergreifend größer als in der Gruppe ohne Zusatz-Weiterbildung, mit einem statistisch signifikanten Unterschied nur in Fallbeispiel 2.2.

Schlüsselt man den oben vorgenommenen Vergleich der Szenarien weiter anhand der Variable „Zusatz-Weiterbildung Palliativmedizin“ auf, so zeigte sich ein signifikanter Unterschied zwischen den Fallbeispielen 1.1 und 1.2 nur in der Gruppe mit Zusatz-Weiterbildung Palliativmedizin $(p<0,00001)$ und nicht in der Gruppe ohne Zusatz-Weiterbildung.

Ebenso verhielt es sich beim Vergleich der Szenarien 2.1 und 2.2. Nur unter den Teilnehmenden mit Zusatz-Weiterbildung Palliativmedizin bestand im Fallbeispiel 2.2 eine signifikant höhere Zustimmung als im Fallbeispiel $2.1(\mathrm{p}<0,00001)$. Beim Vergleich der übrigen Szenarien zeigte sich kein Unterschied zwischen Personen mit und ohne ZusatzWeiterbildung Palliativmedizin.

Item 4: Ich würde dem Wunsch nur nachkommen, wenn sich die Patientin/der Patient bereits unmittelbar in der Sterbephase befindet.

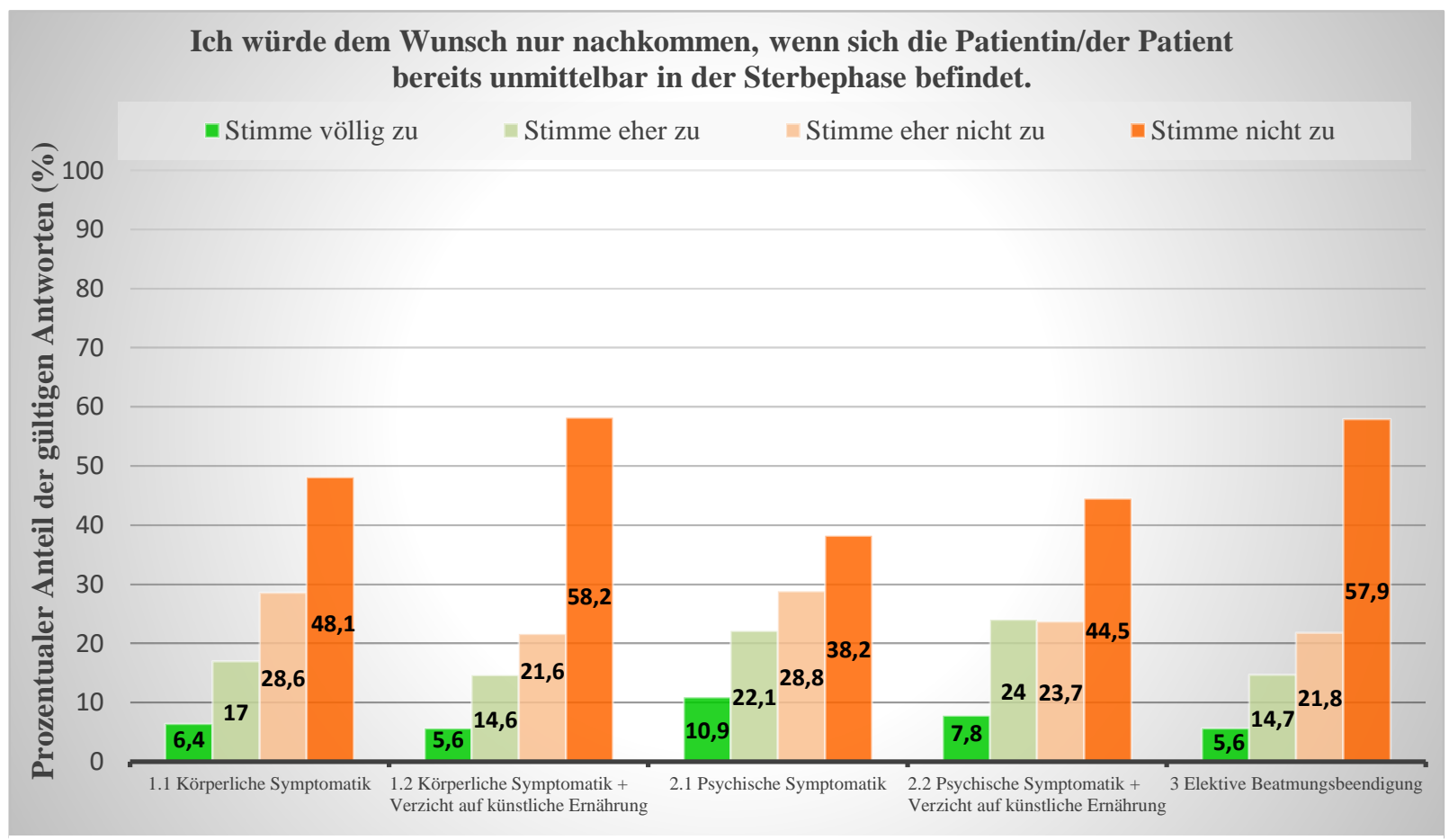

Abbildung 41: Szenarienvergleich Item 4 
Abbildung 41 beinhaltet den Szenarienvergleich bezüglich des vierten Items. Insgesamt $77 \%$ der Antwortenden lehnten in Fallbeispiel 1.1 eine Beschränkung der Palliativen Sedierung auf die unmittelbare Sterbephase ab. Der zusätzliche Wunsch nach Verzicht auf künstliche Ernährung wirkte sich nicht in statistisch signifikantem Ausmaß auf die Zustimmung aus.

Ein ähnliches Bild zeigte sich beim Vergleich der Fallbeispiele 2.1 und 2.2. Auch im Fall der Patientin mit primär psychischer Symptomatik veränderte sich die Zustimmung nicht signifikant, wenn zusätzlich zur Sedierung ein Verzicht auf künstliche Ernährung gewünscht wird.

Wiederum bestand ein signifikanter Zustimmungsunterschied einerseits zwischen den Fallbeispielen 1.1 und 2.1 ( $\mathrm{p}=0,00004)$ und andererseits zwischen den Fallbeispielen 1.2 und 2.2 ( $\mathrm{p}<0,00001)$. Im Fall der Patientin mit psychischer Symptomatik wurde eine Begrenzung der Palliativen Sedierung auf die unmittelbare Sterbephase stärker befürwortet als im Fall der Patientin mit körperlicher Symptomatik.

Weiterhin ergab sich ein signifikanter Zustimmungsunterschied zwischen den Fallbeispielen 2.1 und 3 ( $\mathrm{p}<0,00001)$. Die Beschränkung der Palliativen Sedierung auf die unmittelbare Sterbephase wurde im Fall des Patienten mit Wunsch nach elektiver Beatmungsbeendigung weniger stark befürwortet als im Fall der Patientin mit primär psychischer Symptomatik.

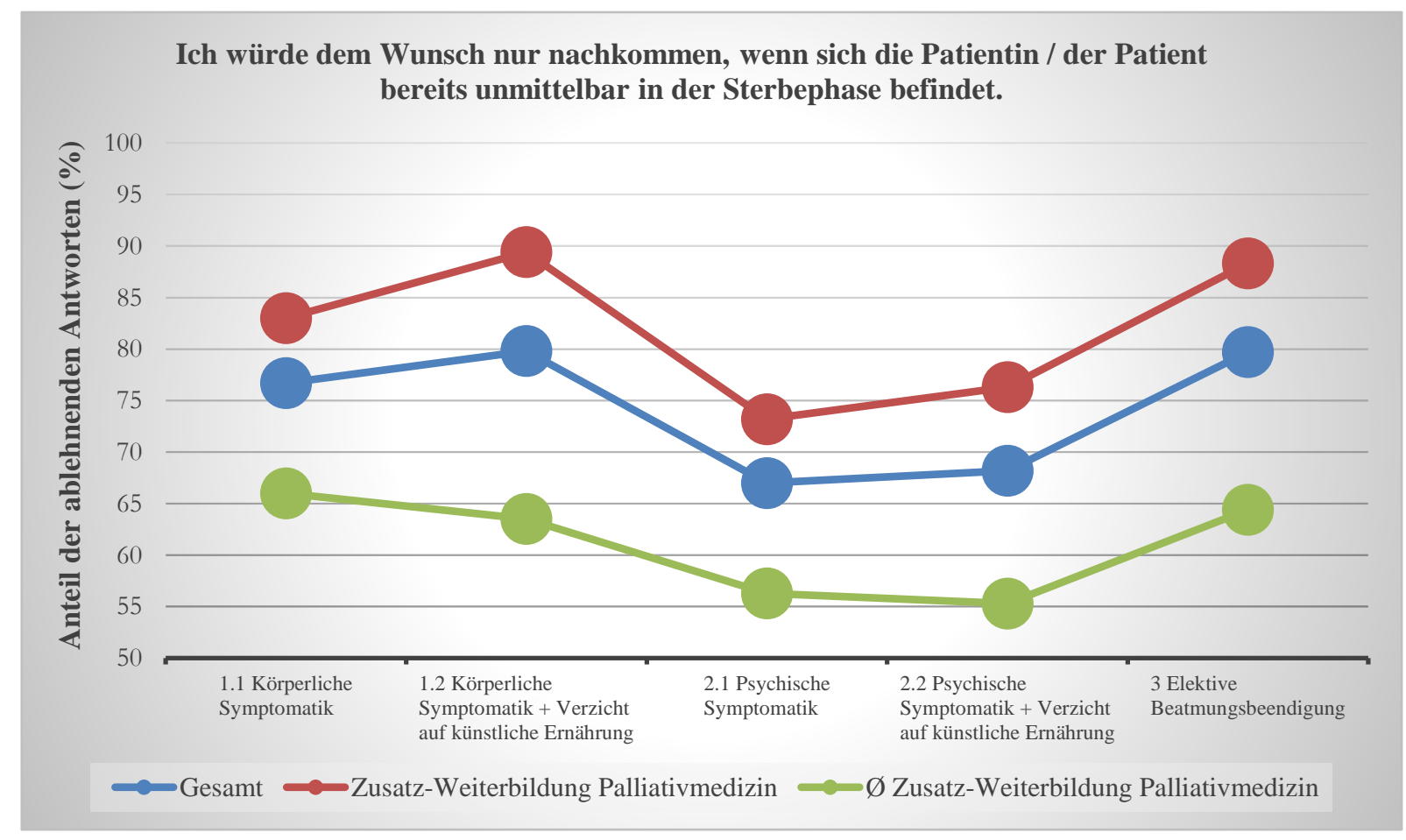

Abbildung 42: Szenarienvergleich mit Differenzierung nach "Zusatz-Weiterbildung Palliativmedizin" Item 4 
Abbildung 42 zeigt den Vergleich der Szenarien und die Differenzierung hinsichtlich der Variable „Zusatz-Weiterbildung Palliativmedizin“. Auf der Ordinatenachse ist in diesem Fall der Anteil der ablehnenden Antworten („Stimme nicht zu“ und „Stimme eher nicht $\mathrm{zu}^{\prime \prime)}$ ) aufgetragen.

Unter den Teilnehmenden mit Zusatz-Weiterbildung Palliativmedizin wurde die Beschränkung der Palliativen Sedierung auf die unmittelbare Sterbephase szenarienübergreifend ablehnender bewertet als von den Antwortenden ohne Zusatz-Weiterbildung, mit statistisch signifikanten Unterschieden in den Fallbeispielen 1.2, 2.1, 2.2 und 3.

Eine weitere Differenzierung anhand der Variable ,Zusatz-Weiterbildung Palliativmedizin“ zeigte, dass innerhalb der Gruppe mit Zusatz-Weiterbildung ein signifikanter Zustimmungsunterschied zwischen den Fallbeispielen 1.1 und 1.2 bestand ( $p=0,00004)$. Der zusätzliche Wunsch der Patientin mit körperlicher Symptomatik nach Verzicht auf künstliche Ernährung führte unter den Teilnehmenden mit Zusatz-Weiterbildung zu einem signifikanten Abfall der Zustimmung. Innerhalb der Gruppe ohne Zusatz-Weiterbildung, sowie in der Gesamtstichprobe, war dies hingegen nicht der Fall.

Ein weiterer Gruppenunterschied konnte beim Vergleich der Szenarien 1.2 und 2.2 festgestellt werden. Die Antwortenden mit Zusatz-Weiterbildung bewerteten die Beschränkung der Palliativen Sedierung auf die unmittelbare Sterbephase im Fall der Patientin mit körperlicher Symptomatik signifikant ablehnender als im Fall der Patientin mit psychischer Symptomatik ( $\mathrm{p}=0,00002)$. In der Gruppe ohne Zusatz-Weiterbildung bestand hingegen kein signifikanter Zustimmungsunterschied.

Schließlich bestanden unter den Befragten mit Zusatz-Weiterbildung Palliativmedizin signifikante Zustimmungsunterschiede einerseits beim Vergleich der Szenarien 1.1 und $3(\mathrm{p}=0,0011)$ und andererseits beim Vergleich der Fallbeispiele 2.1 und $3(\mathrm{p}<0,00001)$. Die Antwortenden mit Zusatz-Weiterbildung reagierten im Fall des Patienten mit Wunsch nach elektiver Beatmungsbeendigung ablehnender als im Fall der Patientin mit körperlicher oder psychischer Symptomatik. In beiden Fällen zeigte sich innerhalb der Gruppe ohne Zusatz-Weiterbildung kein signifikanter Zustimmungsunterschied. 


\subsection{Anmerkungen zur Befragung oder zur Thematik}

Zum Abschluss des Fragebogens konnten die Teilnehmenden im Rahmen eines Freitextfeldes Anmerkungen zur Befragung oder zur Thematik äußern. Insgesamt 61 Personen formulierten eine Freitextantwort. Es wurden viele unterschiedliche Themengebiete angesprochen, was eine Kategorisierung erschwert. Die häufig genannten Themen wurden den folgenden vier Subgruppen zugeordnet. Sämtliche Antworten sind im Anhang zu finden.

\subsubsection{Kritik an der Formulierung der Fragen, Fallvignetten oder der Items}

13 Teilnehmende kritisierten, dass Teile des Fragebogens zu wenig konkret formuliert seien. Mehrfach wurde darauf hingewiesen, dass nicht klar sei, ob unter „künstliche Ernährung“" sowohl Nahrungs- als auch Flüssigkeitszufuhr zu verstehen ist.

Auch wurde die Zusatzfrage zu den Szenarien 1 und 2 wiederholt kritisiert (,Welches Vorgehen würden Sie im Kontext der zuletzt beschriebenen Situation als moralisch belastender empfinden?" $)$. Einige der Antwortenden gaben an, die Frage nicht beantworten zu können, da sie beide Vorgehen nicht als moralisch belastend einstufen würden. Andere Teilnehmende hielten die Formulierung ,moralisch belastend“ im beschriebenen Kontext für problematisch.

Zudem merkten mehrere Personen an, dass die Therapierefraktärität der in den Fallvignetten beschriebenen Symptome nicht deutlich genug herausgestellt werde. Durch die Formulierung sei nicht völlig klar, ob bereits sämtliche Therapiemöglichkeiten ausgeschöpft wurden.

\subsubsection{Hinweis auf die Schwierigkeit, ein ethisch und medizinisch komplexes Thema im Rahmen eines Fragebogens zu behandeln}

Es wurde in 9 Freitextantworten darauf hingewiesen, dass die thematisierten Fragestellungen zu komplex seien, um sie in einem Fragebogen angemessen behandeln zu können und dass diese Form des Abfragens zu unzulässigen Verallgemeinerungen führe. In diesem Kontext wurde mehrfach betont, dass zur Entscheidungsfindung immer individuelle Abwägungen und ausführliche Gespräche mit PatientInnen und Angehörigen stattfinden müssen. 


\subsubsection{Anmerkungen zur Bedeutung einer Patientenverfügung}

Das Thema der Patientenverfügung wurde in sechs Freitextantworten aufgegriffen. Einerseits merkten mehrere Teilnehmende an, dass der Wunsch einwilligungsfähiger PatientInnen unabhängig vom Vorliegen einer Patientenverfügung bindend sei. Andererseits wurde auf weitere Formen der Willensäußerung und der Dokumentation des PatientInnenwunsches verwiesen.

\subsubsection{Angabe von Gründen für fehlendes oder unvollständiges Ausfüllen des Fragebogens}

Wie bereits zu Beginn der Ergebnisdarstellung erwähnt, gaben fünf Teilnehmende an, warum sie den Fragebogen nicht oder nur unvollständig ausgefüllt haben. Dabei stand die fehlende Erfahrung mit ALS-PatientInnen als Begründung im Vordergrund.

Die übrigen 28 Freitextantworten enthielten unterschiedliche Anmerkungen sowohl zum Thema der Palliativen Sedierung bei ALS und deren praktische Umsetzung als auch zur Durchführung der Befragung (siehe Anhang). 


\subsection{Zusammenfassung der Ergebnisse}

Im Rahmen der Befragung wurden 993 ÄrztInnen mit Weiterbildungsermächtigung in den Bereichen Neurologie und/oder Palliativmedizin kontaktiert. Es wurden 296 Fragebögen zurückgesendet und ausgewertet. Knapp $85 \%$ der Antwortenden waren zum Zeitpunkt der Befragung über 45 Jahre alt. Es nahmen mit knapp $70 \%$ der Antwortenden deutlich mehr Männer als Frauen an der Befragung teil, was jedoch der Geschlechterverteilung aller kontaktierten Personen entspricht. Mit $44 \%$ wurde die Neurologie als häufigste absolvierte Facharztausbildung angegeben, gefolgt von der Inneren Medizin mit $27 \%$ und der Anästhesiologie mit $18 \%$. Knapp $63 \%$ der Teilnehmenden gaben an, die Zusatz-Weiterbildung Palliativmedizin absolviert zu haben.

Insgesamt gaben $66 \%$ der Antwortenden an, sich bereits intensiv mit dem Thema der Palliativen Sedierung beschäftigt zu haben. Die teilnehmenden ÄrztInnen mit ZusatzWeiterbildung Palliativmedizin hatten sich deutlich häufiger bereits mit der Thematik auseinandergesetzt als die ÄrztInnen ohne Zusatz-Weiterbildung.

Mehr als drei Viertel aller Antwortenden gaben an, durchschnittlich ein bis drei ALSPatientInnen pro Monat zu behandeln. Weitere $17 \%$ behandelten keine ALS-PatientInnen und knapp $6 \%$ behandelten mehr als vier ALS-PatientInnen pro Monat. Die Teilnehmenden mit Zusatz-Weiterbildung Palliativmedizin behandelten weniger ALS-PatientInnen pro Monat als die Teilnehmenden ohne Zusatz-Weiterbildung.

Weiterhin behandelten $43 \%$ der Antwortenden bereits seit über 15 Jahren ALS-PatientInnen, weitere $37 \%$ gaben an, eine 5- bis 15-jährige Erfahrung mit ALS-PatientInnen aufzuweisen und $11 \%$ der Teilnehmenden hatten keine Erfahrung mit der Behandlung von ALS-PatientInnen. Dabei wiesen die Teilnehmenden ohne Zusatz-Weiterbildung Palliativmedizin eine längere Erfahrung auf als die Vergleichsgruppe mit Zusatz-Weiterbildung.

Insgesamt gaben $42 \%$ der Antwortenden an, bereits eine Palliative Sedierung bei ALSPatientInnen durchgeführt zu haben. Dabei antworteten die Teilnehmenden mit ZusatzWeiterbildung Palliativmedizin deutlich häufiger zustimmend als die ÄrztInnen ohne Zusatz-Weiterbildung. Als Indikationen für die Durchführung einer Palliativen Sedierung bei ALS wurden am häufigsten Luftnot, Angst und Schmerzen genannt.

Im Fallbeispiel 1.1 wird der Fall einer ALS-Patientin mit körperlicher Symptomatik beschrieben, die eine tiefe kontinuierliche Sedierung wünscht. Der Einsatz der Palliativen 
Sedierung in diesem Kontext wurde von den Teilnehmenden insgesamt zustimmend bewertet. Fast alle Antwortenden konnten den Wunsch der Patientin nachvollziehen und $87 \%$ vertraten die Meinung, dass die Patientin ein Recht auf die gewünschten Maßnahmen habe. $85 \%$ der Antwortenden würden eine kontinuierliche tiefe Sedierung erst durchführen, wenn eine vorherige flache oder intermittierende Sedierung nicht erfolgreich war. Weiterhin reagierten mehr als drei Viertel der Teilnehmenden ablehnend darauf, dem Wunsch der Patientin erst nachzukommen, wenn diese sich bereits unmittelbar in der Sterbephase befindet. Auch lehnten es $95 \%$ der teilnehmenden ÄrztInnen ab, generell keine kontinuierliche tiefe Sedierung durchzuführen.

Im Fallbeispiel 1.2 wünscht die Patientin zusätzlich einen Verzicht auf künstliche Ernährung. Wiederum konnten fast alle Teilnehmenden den Wunsch der Patientin nachvollziehen und insgesamt $90 \%$ waren der Ansicht, dass die Patientin ein Recht auf die gewünschten Maßnahmen habe. Knapp zwei Drittel sahen die Dokumentation des Patientenwunsches in einer Patientenverfügung nicht als Voraussetzung für die Durchführung der gewünschten Maßnahmen an. 80 \% lehnten es ab, dem Wunsch erst nachzukommen, wenn sich die Patientin bereits unmittelbar in der Sterbephase befindet. Zudem reagierten $93 \%$ der Teilnehmenden ablehnend auf eine Gleichsetzung der gewünschten Maßnahmen mit einer Tötung auf Verlangen. Insgesamt $57 \%$ der Teilnehmenden vertraten die Meinung, dass der Verzicht auf künstliche Ernährung bei gleichzeitiger kontinuierlicher tiefer Sedierung zu einem früheren Versterben der Patientin führen würde. Schließlich gaben drei Viertel der Antwortenden an, dass eine künstliche Ernährung während einer dauerhaften tiefen Sedierung nicht indiziert sei.

Im Fallbeispiel 2.1 wird eine ALS-Patientin mit starker psychischer Symptomatik beschrieben, die eine tiefe kontinuierliche Sedierung wünscht. $93 \%$ der Antwortenden konnten den Wunsch nachvollziehen und $70 \%$ waren der Ansicht, dass die Patientin ein Recht auf die gewünschten Maßnahmen habe. Knapp $84 \%$ der Teilnehmenden würden dem Wunsch erst nachkommen, wenn vorherige Versuche einer flachen oder intermittierenden Sedierung nicht erfolgreich waren. Weiterhin lehnten zwei Drittel der teilnehmenden ÄrztInnen eine Beschränkung der tiefen kontinuierlichen Sedierung auf die unmittelbare Sterbephase ab. Schließlich lehnten es $93 \%$ der Antwortenden ab, generell keine tiefe kontinuierliche Sedierung durchzuführen.

Im Fallbeispiel 2.2 wünscht die beschriebene Patientin mit psychischer Symptomatik zusätzlich einen Verzicht auf künstliche Ernährung. Insgesamt $96 \%$ der Antwortenden 
konnten den Wunsch nachvollziehen und $78 \%$ gaben an, dass die Patientin ein Recht auf die Durchführung der gewünschten Maßnahmen habe. Zwei Drittel der Teilnehmenden sahen die Dokumentation in einer Patientenverfügung nicht als Voraussetzung für die Durchführung der gewünschten Maßnahmen an. $68 \%$ lehnten eine Beschränkung der gewünschten Maßnahmen auf die unmittelbare Sterbephase ab. Auf eine Gleichsetzung der gewünschten Maßnahmen mit einer Tötung auf Verlangen reagierten $90 \%$ der Teilnehmenden ablehnend. Zudem vertraten $64 \%$ der Antwortenden die Meinung, dass die Durchführung der gewünschten Maßnahmen zu einem vergleichsweise früheren Versterben führen würde. Schließlich gaben $70 \%$ an, dass eine künstliche Ernährung bei kontinuierlicher tiefer Sedierung medizinisch nicht indiziert sei.

Im Rahmen der Zusatzfrage zu den Szenarien 1 und 2 stufte jeweils die Mehrheit der Antwortenden die Sedierung einer bisher noch selbstständig essenden und trinkenden Patientin bei gleichzeitigem Verzicht auf künstliche Ernährung als moralisch belastender ein als den Abbruch einer bereits bestehenden künstlichen Ernährung mit Beginn der Sedierung. Im Rahmen beider Szenarien würden über $30 \%$ der Antwortenden beide Szenarien als gleich belastend einstufen.

Im Fallbeispiel 3 wird ein ALS-Patient im Endstadium der Erkrankung beschrieben, der invasiv beatmet wird und nun aufgrund der fortschreitenden körperlichen Symptomatik eine Beendigung der Beatmung bei gleichzeitiger Palliativer Sedierung wünscht. Alle Teilnehmenden konnten den Wunsch des Patienten nachvollziehen und $95 \%$ gaben an, dass der Patient ein Recht auf die Durchführung der gewünschten Maßnahmen habe. $70 \%$ sahen die Dokumentation in einer Patientenverfügung nicht als Voraussetzung für die Durchführung der gewünschten Maßnahmen an. $80 \%$ der Teilnehmenden lehnten eine Begrenzung der gewünschten Maßnahmen auf die unmittelbare Sterbephase ab. Schließlich reagierten $88 \%$ der Antwortenden ablehnend auf eine Gleichsetzung des gewünschten Vorgehens mit einer Tötung auf Verlangen.

Beim Vergleich des Antwortverhaltens von Personen mit und ohne Zusatz-Weiterbildung Palliativmedizin zeigten sich hinsichtlich einiger Items signifikante Unterschiede, die in den vorangegangenen Kapiteln ausführlich dargestellt wurden. Die Durchführung der Palliativen Sedierung wurde von den Teilnehmenden mit Zusatz-Weiterbildung tendenziell positiver bewertet als von den Teilnehmenden ohne Zusatz-Weiterbildung. Auch lehnten die TeilnehmerInnen mit Zusatz-Weiterbildung eine Beschränkung der Palliati- 
ven Sedierung auf die unmittelbare Sterbephase stärker ab, sie bewerteten den gleichzeitigen Verzicht auf künstliche Ernährung weniger ablehnend und reagierten zustimmender auf den Einsatz der Palliativen Sedierung bei psychischer Symptomatik.

Ebenfalls reagierten die Antwortenden, die bereits eine Palliative Sedierung bei ALS durchgeführt hatten und die ÄrztInnen, die sich im Vorfeld bereits intensiv mit der Thematik beschäftigt hatten, tendenziell zustimmender auf die Durchführung einer Palliativen Sedierung als die entsprechenden Vergleichsgruppen.

Beim Vergleich der Fallbeispiele untereinander fiel auf, dass der in den Szenarien 1.2 und 2.2 zusätzlich geäußerte Wunsch nach Verzicht auf künstliche Ernährung nicht dazu führte, dass die Durchführung der Palliativen Sedierung ablehnender beurteilt wurde. Zum Teil zeigte sich sogar ein Zustimmungszuwachs - insbesondere unter den Teilnehmenden mit Zusatz-Weiterbildung Palliativmedizin - wenn zusätzlich ein Verzicht auf künstliche Ernährung gewünscht wurde. Es zeigte sich aber ein Zustimmungsgefälle von körperlicher zu psychischer Symptomatik. Die Palliative Sedierung wurde im Fall der Patientin mit psychischer Symptomatik deutlich kritischer beurteilt als im Fall der Patientin mit körperlicher Symptomatik. Dabei fiel der Zustimmungsabfall unter den Teilnehmenden ohne Zusatz-Weiterbildung Palliativmedizin größer aus als in der Gruppe mit Zusatz-Weiterbildung. Im Rahmen des Fallbeispiels 3 war die Zustimmung zur Palliativen Sedierung insgesamt am größten. 


\section{Diskussion}

Im Rahmen dieser Arbeit wurde der Einsatz der Palliativen Sedierung bei ALS-PatientInnen aus einer medizinethischen Perspektive beleuchtet. Es wurden sowohl verschiedene Aspekte der Palliativen Sedierung als auch das Krankheitsbild der ALS erörtert und anschließend medizinethische Überlegungen zur Palliativen Sedierung von ALS-PatientInnen angestellt.

Der empirische Teil der Arbeit verfolgte das Ziel, eine Verknüpfung zwischen der medizinischen und medizinethischen Theorie einerseits und der klinischen Praxis andererseits herzustellen. Dazu wurden ÄrztInnen mit Weiterbildungsermächtigung Neurologie und/oder Palliativmedizin zu ihren Erfahrungen und Einstellungen gegenüber Palliativer Sedierung bei ALS befragt.

Im Folgenden werden zunächst die Ergebnisse der Befragung anhand der in Kapitel 3 formulierten Forschungsfragen diskutiert und mit dem aktuellen Stand der Forschung verknüpft. Zudem sollen mögliche Konsequenzen für den medizinethischen Diskurs und die klinische Praxis angesprochen werden. Anschließend werden die Stärken und Schwächen der durchgeführten Untersuchung analysiert.

\subsection{Diskussion der Forschungsfragen}

\subsubsection{Kommt die Palliative Sedierung bei der Behandlung von ALS-PatientInnen zum Einsatz?}

Knapp $42 \%$ der Antwortenden gaben an, bereits eine Palliative Sedierung bei einem ALS-Patienten bzw. einer ALS-Patientin durchgeführt zu haben. Als Indikationen für die Durchführung der Palliativen Sedierung bei ALS wurden vorrangig Dyspnoe und Angst genannt. Vor dem Hintergrund der aktuellen Literatur, in der ein Einsatz der Palliativen Sedierung bei ALS eher selten thematisiert wird, ist diese Zahl erstaunlich hoch. Es stellt sich deshalb die Frage, ob die Antwortenden - trotz der auf dem Fragebogen aufgeführten Definition der Palliativen Sedierung - unter dem Begriff auch andere Formen der Sedierung zusammengefasst haben und die Frage deshalb zustimmend beantwortet haben. Es ist jedoch auch denkbar, dass Sedierungspraktiken, die einer Palliativen Sedierung entsprechen, in der Praxis tatsächlich häufiger angewendet werden als die Literatur vermuten lässt - gegebenenfalls ohne, dass diese Maßnahmen explizit als Palliative Sedierung bezeichnet werden. 
Interpretiert man das Ergebnis dahingehend, dass tatsächlich bis zu $42 \%$ der Antwortenden bereits eine Palliative Sedierung bei ALS-PatientInnen durchgeführt haben, so ist dies aus medizinethischer Sicht zunächst positiv zu bewerten. Es besteht in der medizinethischen Literatur weitgehend Konsens darüber, dass die Palliative Sedierung eine angemessene Maßnahme ist, um belastende, therapierefraktäre Symptome zu lindern (Cherny et al. 2009; Neitzke et al. 2010; Oechsle et al. 2017).

Vor allem im Kontext der Behandlung von KrebspatientInnen wird die Palliative Sedierung in der medizinethischen Literatur beschrieben und in der aktuellen S3-Leitlinie Palliativmedizin als adäquate Maßnahme zur Linderung therapierefraktärer Symptome bei KrebspatientInnen genannt (Jaspers et al. 2012; AWMF 2018). Es wäre deshalb im Hinblick auf das Prinzip der Gerechtigkeit medizinethisch nicht zu rechtfertigen, nichtonkologischen PatientInnen mit entsprechendem Bedarf eine Palliative Sedierung vorzuenthalten.

Die vorliegenden Ergebnisse deuten darauf hin, dass ALS-PatientInnen nicht grundsätzlich vom Einsatz einer Palliativen Sedierung ausgeschlossen werden. Es kann jedoch anhand der Befragung keine Aussage darüber getroffen werden, ob den aktuellen Empfehlungen zu Indikationsstellung und Durchführung - wie sie beispielsweise die EAPC, die AG Ethik am Lebensende in der AEM und das CCC-Netzwerk formulieren entsprochen wurde. Eine detailliertere Analyse der Sedierungspraktiken bei ALS könnte dementsprechend Gegenstand künftiger Erhebungen sein.

\subsubsection{Inwiefern würden behandelnde ÄrztInnen dem Wunsch von ALS- PatientInnen nach kontinuierlicher und tiefer Sedierung zustimmen, um einerseits körperliche und andererseits psychische Symptome zu lindern?}

Diese Forschungsfrage spricht verschiedene Aspekte an, die im Rahmen der Befragung thematisiert wurden. Zunächst stellte sich die Frage, ob die teilnehmenden ÄrztInnen überhaupt eine tiefe kontinuierliche Sedierung bei ALS-PatientInnen durchführen würden. In diesem Kontext wurde auch nach dem angemessenen Zeitpunkt für eine Palliative Sedierung gefragt. Weiterhin wurde analysiert, wie die Palliative Sedierung einerseits im Rahmen körperlicher Symptome und andererseits im Rahmen psychischer Symptome von den Teilnehmenden bewertet wurde.

Die Ergebnisse der Befragung zeigen, dass unter $5 \%$ der Antwortenden im Falle einer ALS-Patientin mit körperlicher Symptomatik generell keine tiefe kontinuierliche Sedierung durchführen würden. Im Falle einer ALS-Patientin mit psychischer 
Symptomatik waren es knapp $8 \%$. Die deutliche Mehrheit der Teilnehmenden sah die Palliative Sedierung bei ALS also grundsätzlich als adäquate Maßnahme zur Leidenslinderung an. Diese Ergebnisse entsprechen einer im Jahr 2002 durchgeführten Befragungen der Deutschen Gesellschaft für Palliativmedizin, in der $94 \%$ der teilnehmenden ÄrztInnen eine Palliative Sedierung zur Linderung unerträglichen Leids befürworteten (Müller-Busch et al. 2004).

Gleichzeitig wurde die Palliative Sedierung bei psychischer Symptomatik kritischer bewertet als bei körperlicher Symptomatik. Dies war unter den Teilnehmenden ohne Zusatz-Weiterbildung Palliativmedizin stärker der Fall als unter den Teilnehmenden mit Zusatz-Weiterbildung. Die Antwortenden konnten den Wunsch nach Palliativer Sedierung bei Vorliegen einer körperlichen Symptomatik stärker nachvollziehen. Auch sprachen die Teilnehmenden einer Patientin mit körperlicher Symptomatik häufiger ein Recht auf die Durchführung der gewünschten Maßnahmen zu als einer Patientin mit psychischer Symptomatik. Ebenfalls gaben die Antwortenden im Kontext psychischer Symptomatik häufiger an, eine Palliative Sedierung erst in der unmittelbaren Sterbephase durchführen zu wollen.

Diese Ergebnisse überraschen vor dem Hintergrund des aktuellen medizinethischen Diskurses nicht. In zahlreichen Veröffentlichungen wird zwar betont, dass die Palliative Sedierung nicht grundsätzlich auf PatientInnen mit körperlicher Symptomatik beschränkt sein sollte. Doch wird die Indikationsstellung bei psychischen Symptomen als deutlich schwieriger angesehen. Insbesondere seien die Therapierefraktärität schwerer zur beurteilen und die Dynamik des Krankheitsverlaufs schlechter abzuschätzen (Cherny et al. 2009; Neitzke et al. 2010).

Trotz dieses Zustimmungsgefälles, sollte erwähnt werden, dass auch im Falle einer Patientin mit psychischer Symptomatik noch immer die überwiegende Mehrheit der Teilnehmenden den Wunsch nach kontinuierlicher tiefer Sedierung nachvollziehen konnte und $70 \%$ der Befragten gaben an, dass die Patientin ein Recht auf eine Palliative Sedierung habe.

Diese Zahlen unterscheiden sich von den Ergebnissen, die Simon et al. (2007) im Rahmen einer Befragung von Mitgliedern der Akademie für Ethik in der Medizin erhoben haben. Die Sedierung eines sterbenden Patienten, der an therapierefraktären psychischen Symptomen leidet, wurde von $61 \%$ der Teilnehmenden mit medizinischem Hintergrund als zulässig und von $23 \%$ als nicht zulässig beurteilt (Simon et al. 2007). Wie erwähnt 
gaben im Rahmen der vorliegenden Arbeit nur $8 \%$ der teilnehmenden ÄrztInnen an, bei einer Patientin mit psychischer Symptomatik grundsätzlich keine tiefe kontinuierliche Sedierung durchführen zu wollen. Ein Teil dieser Zustimmungsunterschiede mag durch die unterschiedliche Formulierung der Fragen und Szenarien erklärbar sein. Auch unterscheiden sich die Stichproben, da Simon et al. (2007) gezielt Mitglieder der Akademie für Ethik befragt haben. Es ist jedoch auch denkbar, dass sich innerhalb der letzten zehn Jahren eine größere Akzeptanz gegenüber Palliativer Sedierung zur Linderung psychischer Symptomatik entwickelt hat.

\subsubsection{Inwiefern beeinflusst der gleichzeitige Wunsch nach Verzicht auf künstliche Ernährung die Bewertung der Palliativen Sedierung?}

In der medizinethischen Literatur wird der Einsatz einer tiefen kontinuierlichen Sedierung bei gleichzeitigem Verzicht auf künstliche Ernährung besonders kontrovers diskutiert, da dieses Vorgehen in der Regel zu einem Versterben der Betroffenen innerhalb einiger Tage führt (Lipuma 2013). Orentlicher (1997) setzt dieses Vorgehen sogar explizit mit Euthanasie gleich.

Hallenbeck (2000) und Simon (2008) argumentieren hingegen, dass die Entscheidung für oder gegen lebenserhaltende Maßnahmen unabhängig von der Entscheidung für oder gegen eine Palliative Sedierung getroffen werden müsse. Jeder Mensch habe das Recht, lebenserhaltende Maßnahme abzulehnen und diese Entscheidung dürfe nicht die Indikationsstellung der Palliativen Sedierung beeinflussen. Auch in der Rahmenleitlinie der EAPC stellen Cherny et al. (2009) fest:

„The decision about artificial hydration/nutrition therapy is independent of the decision about sedation itself. Whether artificial hydration/nutrition therapy is performed should be individually decided through comprehensive evaluation of the patient's wishes and the estimated benefits/harms in light of the treatment aim (palliation of suffering)".

Es wird in der Rahmenleitlinie der EAPC jedoch ebenfalls darauf hingewiesen, dass die Meinungen und Praktiken diesbezüglich variieren (Cherny et al. 2009).

Vor dem Hintergrund dieser Diskussion, erstaunen die vorliegenden Ergebnisse hinsichtlich Palliativer Sedierung und künstlicher Ernährung. Die Durchführung der Palliativen Sedierung wurde in keinem Fallbeispiel ablehnender beurteilt, wenn gleichzeitig ein Verzicht auf künstliche Ernährung gewünscht wird. Ganz im Gegenteil 
sprachen die Teilnehmenden sowohl einer Patientin mit körperlicher als auch einer Patientin mit psychischer Symptomatik eher ein Recht auf eine Palliative Sedierung zu, wenn gleichzeitig der Wunsch nach Verzicht auf künstliche Ernährung besteht (siehe Kapitel 5.8).

Eine Gleichsetzung der tiefen kontinuierlicher Sedierung bei Verzicht auf künstliche Ernährung mit einer Tötung auf Verlangen lehnten über $90 \%$ der Teilnehmenden ab. Dieses Ergebnis überrascht, da im Rahmen einer Befragung von van Oorschot et al. (2005) etwa $25 \%$ der teilnehmenden ÄrztInnen bereits eine Beendigung der Flüssigkeitszufuhr ohne gleichzeitige Palliative Sedierung als aktive Sterbehilfe klassifizierten. Wie in Kapitel 2.2.2 erörtert, ist ein Verzicht auf künstliche Ernährung bei Palliativer Sedierung aus aktueller medizinethischer und rechtlicher Perspektive nicht mit einer Tötung auf Verlangen gleichzusetzen. Jedoch ist es vor dem Hintergrund der kontroversen medizinethischen Diskussion durchaus denkbar, dass ÄrztInnen aufgrund rechtlicher Bedenken vor der Durchführung einer Palliativen Sedierung zurückschrecken, insbesondere wenn diese mit einem Verzicht auf künstliche Ernährung verbunden ist. Dies lässt sich aus den vorliegenden Ergebnissen jedoch nicht ableiten.

Gleichzeitig gaben im Falle der Patientin mit körperlicher Symptomatik $57 \%$ und im Falle der Patientin mit psychischer Symptomatik $64 \%$ der Antwortenden an, dass ein Verzicht auf künstliche Ernährung bei Palliativer Sedierung zu einem früheren Versterben führen würde. Wie bereits beschrieben, wird die Durchführung dieser Maßnahmen trotzdem von der überwiegenden Mehrheit der Teilnehmenden positiv bewertet. Ein Großteil der Antwortenden scheint demnach die Meinung zu vertreten, dass die Linderung stark belastender Symptome Vorrang vor einer Lebensverlängerung hat. Auch wenn dieses Thema weiterhin kontrovers diskutiert wird, so entspricht diese Ansicht in weiten Teilen dem aktuellen medizinethischen Diskurs (AWMF 2018; Nationaler Ethikrat 2006; Simon 2006).

Außerdem vertraten etwa $70 \%$ der Teilnehmenden die Meinung, dass eine künstliche Ernährung während einer tiefen kontinuierlichen Sedierung nicht indiziert sei. Dies entspricht den Empfehlungen der AG Ethik am Lebensende in der AEM, die einen Abbruch lebenserhaltender Maßnahmen im Rahmen einer Stufe II Sedierung vorschlagen (Neitzke et al. 2010). 


\subsubsection{Wie beurteilen behandelnde ÄrztInnen den PatientInnenwunsch nach elektiver Beatmungsbeendigung bei gleichzeitiger Palliativer Sedierung?}

Der Einsatz der Palliativen Sedierung wurde im Rahmen einer elektiven Beatmungsbeendigung überwiegend positiv bewertet. Alle Teilnehmenden konnten den Wunsch des ALS-Patienten nachvollziehen und $95 \%$ vertraten die Meinung, dass der Patient ein Recht auf Palliative Sedierung bei elektiver Beatmungsbeendigung habe. Außerdem lehnten $80 \%$ der Antwortenden eine Beschränkung der gewünschten Maßnahmen auf die unmittelbare Sterbephase ab und $88 \%$ würden das Vorgehen nicht mit einer Tötung auf Verlangen gleichsetzen.

Auch diese Ergebnisse waren unter Berücksichtigung vorangegangener Befragungen nicht unbedingt zu erwarten. In Studien von van Oorschot et al. (2005) und Weber et al. (2001) sahen 40-50 \% der teilnehmenden ÄrztInnen eine Beendigung künstlicher Beatmung als aktive Sterbehilfe an. Es ist jedoch durchaus denkbar, dass nicht alle Teilnehmenden mit den Definitionen der verschiedenen Formen der Sterbehilfe vertraut sind und dass der Begriff „Tötung auf Verlangen“ im Rahmen der vorliegenden Befragung uneinheitlich interpretiert wurde. Auf eine solche Unsicherheit bezüglich der Begrifflichkeiten weisen auch die Studien von Weber et al. (2001) und van Oorschot et al. (2005) hin. Ebenfalls wurde die Problematik der Begriffsdefinition im Rahmen der Freitextantworten der vorliegenden Umfrage angesprochen (siehe Anhang).

Geht man jedoch davon aus, dass tatsächlich ein Großteil der Befragten eine elektive Beatmungsbeendigung nicht mit einer Tötung auf Verlangen gleichsetzt, so ist dies aus medizinethischer Perspektive weitgehend positiv zu bewerten. Dem Zwei-Säulen-Modell aus Indikation und Einwilligung folgend, hat jeder Mensch das Recht, medizinische Maßnahmen abzulehnen. Die Durchführung einer medizinischen Maßnahme gegen den Willen der PatientInnen ist zudem rechtlich als Körperverletzung einzustufen (Nationaler Ethikrat 2006). Auch erscheint eine solche Entscheidung im Hinblick auf das von Beauchamp und Childress (2001) formulierte Prinzip der Autonomie legitim. Jedoch ist eine elektive Beatmungsbeendigung im Kontext einer fortgeschrittenen ALS-Erkrankung nicht ohne palliative, sedierende Maßnahmen zumutbar (Meyer et al. 2008). Eine Ablehnung der Palliativen Sedierung während der Beatmungsbeendigung würde es den meisten Betroffenen dementsprechend unmöglich machen, ihr Recht auf Beatmungsbeendigung umzusetzen. 


\subsubsection{Inwiefern wird das Antwortverhalten durch die Berufserfahrung und die Aus- und Weiterbildung der teilnehmenden ÄrztInnen beeinflusst?}

Im Rahmen dieser Forschungsfrage lag der Fokus der Auswertung auf dem Vergleich von ÄrztInnen mit und ohne Zusatz-Weiterbildung Palliativmedizin. Die Auswertung der Ergebnisse ergab, dass $52 \%$ der Teilnehmenden mit Zusatz-Weiterbildung und nur $26 \%$ der Teilnehmenden ohne Zusatz-Weiterbildung Palliativmedizin bereits eine Palliative Sedierung bei ALS durchgeführt hatten. Gleichzeitig behandelten zum Zeitpunkt der Befragung die Teilnehmenden ohne Zusatz-Weiterbildung mehr ALS-PatientInnen und gaben eine längere Berufserfahrung mit ALS-PatientInnen an.

Diese Ergebnisse überraschen nicht, da zur Teilnahme an der Befragung ÄrztInnen mit Weiterbildungsermächtigung Neurologie und/oder Palliativmedizin ausgewählt wurden. Die Gruppe der Teilnehmenden ohne Zusatz-Weiterbildung Palliativmedizin setzte sich demnach vollständig aus ÄrztInnen mit Weiterbildungsermächtigung Neurologie zusammen. Erwartungsgemäß wird die Behandlung von ALS-PatientInnen primär von ÄrztInnen mit Weiterbildungsermächtigung Neurologie durchgeführt, während die Palliative Sedierung von ALS-PatientInnen häufiger durch ÄrztInnen mit Weiterbildungsermächtigung Palliativmedizin erfolgt.

Auch zeigte sich, dass die teilnehmenden ÄrztInnen mit Zusatz-Weiterbildung Palliativmedizin eine Palliative Sedierung von ALS-PatientInnen tendenziell positiver bewerteten als die Teilnehmenden ohne Zusatz-Weiterbildung. Sie gaben beispielsweise seltener an, generell keine kontinuierliche tiefe Sedierung durchführen zu wollen. Das Vorliegen einer Patientenverfügung wurde von den Antwortenden mit ZusatzWeiterbildung als weniger wichtig beurteilt und eine Begrenzung der Palliativen Sedierung auf die unmittelbare Sterbephase wurde stärker abgelehnt. Ebenfalls setzten sie eine Palliative Sedierung bei gleichzeitigem Verzicht auf künstliche Ernährung seltener mit einer Tötung auf Verlangen gleich als die Teilnehmenden ohne ZusatzWeiterbildung. Auch ist der Zustimmungsunterschied zwischen körperlicher und psychischer Symptomatik unter den Antwortenden mit Zusatz-Weiterbildung kleiner als in der Vergleichsgruppe ohne Zusatz-Weiterbildung. Zudem vertraten die Antwortenden mit Zusatz-Weiterbildung häufiger die Meinung, dass eine künstliche Ernährung bei tiefer kontinuierlicher Sedierung nicht indiziert sei.

Etwas weniger häufig zeigten sich diese Gruppenunterschiede auch zwischen den teilnehmenden ÄrztInnen mit und ohne vorherige intensive Beschäftigung mit dem 
Thema der Palliativen Sedierung und für die Teilnehmenden mit und ohne praktische Erfahrung mit Palliativer Sedierung von ALS-PatientInnen.

Es überrascht nicht, dass diejenigen ÄrztInnen, die sich im Rahmen ihrer Aus- und Weiterbildung bereits mit dem Thema der Palliativen Sedierung beschäftigt haben und gegebenenfalls sogar praktische Erfahrungen auf dem Gebiet aufweisen, zustimmender auf die Durchführung einer Palliativen Sedierung bei ALS reagieren. Weiterhin stimmen die Einschätzungen dieser ÄrztInnen tendenziell stärker mit den im aktuellen medizinethischen Diskurs vorherrschenden Auffassungen überein. Beispielsweise wird das Vorliegen einer Patientenverfügung aus medizinethischer und rechtlicher Perspektive nicht als notwendig erachtet, insofern die Betroffenen noch einwilligungsfähig sind und ihre Wünsche klar formulieren. Ebenso wird ein Verzicht auf künstliche Ernährung bei Palliativer Sedierung sowohl aus medizinethischer als auch aus rechtlicher Sicht nicht mit einer Tötung auf Verlangen gleichgesetzt.

\subsection{Stärken und Schwächen der Untersuchung}

Eine Stärke der vorliegenden Untersuchung besteht darin, dass erstmals eine bundesweite repräsentative Befragung von NeurologInnen und PalliativmedizinerInnen zum Thema der Palliativen Sedierung bei ALS durchgeführt wurde. In der aktuellen medizinethischen Literatur finden sich nur vereinzelt Berichte über den konkreten Einsatz der Palliativen Sedierung bei neurologischen Erkrankungen wie der ALS. Die vorliegende Arbeit liefert Hinweise darauf, dass die Palliative Sedierung bei ALS zum Einsatz kommt und von den behandelnden ÄrztInnen als sinnvolle Maßnahme zur Linderung therapierefraktärer Symptome angesehen wird. Diese Ergebnisse könnten zum Anlass genommen werden, um den Einsatz der Palliative Sedierung im Rahmen nicht-onkologischer Erkrankungen stärker in den Fokus zu rücken.

Die Freitextfrage am Ende des Fragebogens, im Rahmen derer Anmerkungen zur Thematik oder zur Befragung formuliert werden konnten, weist auf einige methodische Schwächen hin. Es wurde angemerkt, dass einige Fragen zu wenig konkret formuliert waren. Beispielsweise war nicht für alle Teilnehmenden eindeutig, ob unter ,künstlicher Ernährung“ auch eine Flüssigkeitszufuhr zusammengefasst war. Auch wurde mehrfach angemerkt, dass bei der Beschreibung der Fallbeispiele die Therapierefraktärität der Symptome nicht eindeutig genug herausgestellt wurde. Es war offensichtlich nicht allen 
Teilnehmenden klar, ob bereits sämtliche alternative Therapiemöglichkeiten ausgeschöpft wurden.

Außerdem wurde die Zusatzfrage zu den Szenarien 1 und 2 wiederholt kritisiert („Welches Vorgehen würden Sie im Kontext der zuletzt beschriebenen Situation als moralisch belastender empfinden?“). Einige TeilnehmerInnen gaben an, diese Frage nicht beantworten zu können, da sie keines der Szenarien als moralisch belastend bewerten würden.

Zwar wurde zu Beginn des Fragebogens eine Definition der Palliativen Sedierung formuliert, um eine gemeinsame Grundlage für alle Teilnehmenden zu schaffen. Jedoch kann anhand der Befragung keine Aussage darüber getroffen werden, welche Formen der Palliativen Sedierung von den Teilnehmenden tatsächlich bereits eingesetzt wurden. Es ist durchaus denkbar, dass auch Sedierungspraktiken, die streng genommen nicht der Definition der Palliativen Sedierung entsprechen, von den Teilnehmenden bei der Beantwortung des Fragebogens mitberücksichtigt wurden.

Schließlich muss darauf hingewiesen werden, dass die Thematik der Palliativen Sedierung von ALS-PatientInnen sehr komplex ist und im Rahmen einer Fragebogenstudie nur annäherungsweise erfasst werden kann. Die im Fragebogen angesprochenen Entscheidungen erfordern im klinischen Alltag eine sorgfältige Abwägung jedes Einzelfalls unter Einbezug eines multidisziplinären Teams. Ein Fragebogen kann dieser Komplexität nie völlig gerecht werden. Auch diese Problematik wurde im Rahmen der Freitextantworten mehrfach angesprochen. 


\section{$5 \quad$ Fazit}

In dieser Arbeit wurde der Einsatz der Palliativen Sedierung bei ALS-PatientInnen diskutiert. Die Palliative Sedierung ist als Maßnahme zur Linderung belastender therapierefraktärer Symptome weitgehend anerkannt. Dennoch bestehen in der medizinethischen Literatur einige Kontroversen um die Thematik, die insbesondere die Indikationsstellung, den angemessenen Zeitpunkt für eine Sedierung und den gleichzeitigen Verzicht auf lebenserhaltende Maßnahmen betreffen.

Während die Palliative Sedierung zumeist im Kontext onkologischer Erkrankungen thematisiert wird, steht im Rahmen dieser Arbeit eine neurologische Erkrankung - die ALS - im Fokus. Aufgrund der charakteristischen Symptome der ALS, wie Dysphagie, Dysarthrie und respiratorische Insuffizienz, erscheint der Einsatz einer Palliative Sedierung durchaus denkbar. Jedoch finden sich aktuell nur wenige Hinweise darauf in der medizinischen und medizinethischen Literatur.

Aufbauend auf diesen theoretischen Überlegungen, wurden fünf Forschungsfragen entwickelt, die anschließend im Rahmen einer Fragebogenstudie adressiert wurden. Aufgrund der nur sporadischen Hinweise in der medizinischen und medizinethischen Literatur, stellte sich zunächst die Frage, ob eine Palliative Sedierung von ALS-PatientInnen bereits zum Einsatz kommt. Anknüpfend an die aktuellen medizinethischen Kontroversen, sollte zudem erhoben werden, ob die Indikationsstellung bei psychischer Symptomatik als problematischer eingestuft wird als bei körperlicher Symptomatik und ob ein gleichzeitiger Verzicht auf künstliche Ernährung die Indikationsstellung beeinflusst. Weiterhin wurde erfragt, wie die behandelnden ÄrztInnen eine elektive Beatmungsbeendigung bei gleichzeitiger Palliativer Sedierung bewerten. Schließlich sollte analysiert werden, ob die Zustimmung zur Palliativen Sedierung durch bestimmte Kontextvariablen - insbesondere durch die Zusatz-Weiterbildung Palliativmedizin - beeinflusst wird.

Im Rahmen der Fragebogenstudie zeigte sich, dass die Palliative Sedierung bei ALS zum Teil bereits eingesetzt wird und in erster Linie von ÄrztInnen mit Zusatz-Weiterbildung Palliativmedizin durchgeführt wird. Weiterhin wurde deutlich, dass nur sehr wenige TeilnehmerInnen eine tiefe kontinuierliche Sedierung bei ALS-PatientInnen grundsätzlich ablehnten. Die Palliative Sedierung wurde vom Großteil der Antwortenden prinzipiell als adäquate Maßnahme zur Linderung therapierefraktärer Symptome bei ALS angesehen. Die Zustimmung zur Palliativen Sedierung war im Kontext körperlicher Symptome grö- 
Ber als bei psychischer Symptomatik. Der zusätzliche Wunsch nach Verzicht auf künstliche Ernährung wirkte sich jedoch nicht negativ auf die Zustimmung zur Palliativen Sedierung aus. Auch die elektive Beatmungsbeendigung bei gleichzeitiger Palliativer Sedierung wurde von der Mehrzahl der Teilnehmenden zustimmend bewertet. Schließlich zeigte sich, dass die Antwortenden mit Zusatz-Weiterbildung Palliativmedizin der Palliativen Sedierung insgesamt positiver gegenüberstanden als die teilnehmenden ÄrztInnen ohne Zusatz-Weiterbildung.

Aus medizinethischer Perspektive ist es zu begrüßen, dass die Palliative Sedierung auch nicht-onkologischen PatientInnen mit entsprechendem Bedarf angeboten wird. Auch weisen die vorliegenden Ergebnisse auf die Bedeutung der palliativmedizinischen Mitbehandlung von ALS-PatientInnen hin. Die Teilnehmenden mit Zusatz-Weiterbildung Palliativmedizin standen der Palliativen Sedierung deutlich offener gegenüber und ihre Einschätzungen bezüglich Palliativer Sedierung entsprachen stärker den im aktuellen medizinethischen Diskurs vertretenen Auffassungen. Demnach ist es auch positiv zu bewerten, dass die Palliative Sedierung in den meisten Fällen durch ÄrztInnen mit Zusatz-Weiterbildung Palliativmedizin durchgeführt wird.

Anhand der vorliegenden Befragung kann jedoch keine Aussage darüber getroffen werden, inwieweit die in der Literatur beschriebenen Empfehlungen zur Durchführung der Palliativen Sedierung berücksichtigt werden. Es ist denkbar, dass die konkreten Sedierungspraktiken variieren. Die Formulierung von konkreten Leitlinien auf lokaler Ebene könnte dazu beitragen, die Durchführung zu standardisieren und mehr Sicherheit für behandelnde ÄrztInnen zu schaffen. 


\section{Anhang}

\subsection{Fragebogen}

\begin{tabular}{|l|l|l|}
\hline EvaSys & Medizinethische Bewertung der Palliativen Sedierung bei ALS-Patientinnen und -Patienten & E Electric Paper \\
\hline \hline Akademie für Ethik in der Medizin & A. Simon, B. Alt-Epping, L.Salzmann \\
Universitätsmedizin Göttingen & Palliative Sedierung bei ALS \\
\hline
\end{tabular}

Bitte so markieren: $\square \mathbb{X \square \square} \square$ Bitte verwenden Sie einen Kugelschreiber oder nicht zu starken Filzstift. Dieser Fragebogen wird maschinell erfasst. Korrektur: $\quad \square \square \square \bigotimes \square$ Bitte beachten Sie im Interesse einer optimalen Datenerfassung die links gegebenen Hinweise beim Ausfüllen.

Der folgende Fragebogen thematisiert den Einsatz einer Palliativen Sedierung in der Behandlung von ALSPatientinnen und ALS-Patienten.

Das Handlungskonzept der Palliativen Sedierung ist definiert als „der überwachte Einsatz von Medikamenten mit dem Ziel einer verminderten oder aufgehobenen Bewusstseinslage (Bewusstlosigkeit), um die Symptomlast in anderweitig therapierefraktären Situationen in einer für Patienten, Angehörigen und Mitarbeitern ethisch akzeptablen Weise zu reduzieren" (European Association for Palliative Care 2010).

Bitte schicken Sie den ausgefüllten Fragebogen bis zum 24.06.2018 zusammen mit dem Deckblatt an die auf dem Deckblatt angegebene Adresse.

Sind Sie mit dem Begriff der Palliativen Sedierung vertraut?

$\square$ Ich habe den Begriff bisher noch nie gehört.

$\square$ Mir ist der Begriff bekannt, ich habe mich mit dem Thema jedoch noch nicht beschäftigt.
Mir ist der Begriff bekannt und ich habe mich bereits ein wenig mit dem Thema beschäftigt.

$\square$ Mir ist der Begriff bekannt und ich

habe mich bereits intensiv mit dem

Thema beschäftigt.

Wie viele ALS-Patientinnen und ALS-Patienten behandeln Sie im Durchschnitt pro Monat?
$\square$ keine
$\square$ unter 3
$\square 4-10$

$\square$ über 10

Wie lange behandeln Sie schon ALS-Patientinnen und ALS-Patienten?
$\square$ Bisher keine Erfahrung
$\square$ unter 5 Jahre
$\square 5$ - 10 Jahre
$\square$ 10- 15 Jahre
$\square$ über 15 Jahre

Haben Sie bereits eine Palliative Sedierung bei einer ALS-Patientin bzw. einem ALS-Patienten durchgeführt?

$\square \mathrm{Ja}$ $\square$ Nein

Wenn ja, bei welchen therapierefraktären Symptomen? 
\begin{tabular}{l|l|l|l|l} 
EvaSys & Medizinethische Bewertung der Palliativen Sedierung bei ALS-Patientinnen und -Patienten & Electric Paper \\
\hline
\end{tabular}

\section{Fallbeispiel 1}

Sie behandeln eine ALS-Patientin im fortgeschrittenen Krankheitsstadium. Die Patientin hat starke körperliche Symptome (Dyspnoe, Pseudohypersalivation, Dysphagie, Dysarthrie, Schmerzen durch Spastik und Muskelkrämpfe), die bisher nicht in ausreichendem Maße gelindert werden konnten. Die Patientin ist dadurch offensichtlich stark belastet.

Die Patientin ist einwilligungsfähig und wünscht aufgrund der refraktären körperlichen Symptomatik eine kontinuierliche und tiefe Sedierung.

Bitte geben Sie für jede Aussage an, inwieweit Sie dieser zustimmen.

Der Wunsch der Patientin ist für mich nachvollziehbar.

Die Patientin hat ein Recht darauf, dass ihrem Wunsch entsprochen wird.

Ich würde dem Wunsch nur nachkommen, wenn vorherige Versuche einer flachen bzw. intermittierenden Sedierung keine ausreichende Symptomlinderung erzielen konnten.

Ich würde dem Wunsch nur nachkommen wenn sich die Patientin bereits unmittelbar in der Sterbephase befindet.

Ich würde generell keine kontinuierliche tiefe Sedierung durchführen.

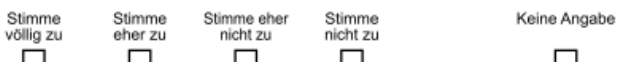

$\square$
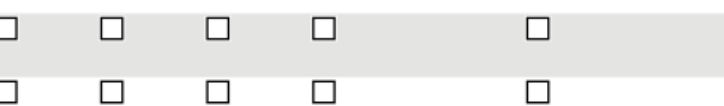

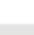


\begin{tabular}{l|l|l|l} 
EvaSys & Medizinethische Bewertung der Palliativen Sedierung bei ALS-Patientinnen und -Patienten & Eler \\
\hline
\end{tabular}

\section{Fallbeispiel 2}

Sie behandeln eine ALS-Patientin im fortgeschrittenen Krankheitsstadium. Die Patientin weist starke psychische Symptome (Angst, Panik, Depression) und Belastungen im Sinne existenziellen Leids auf. Diese Symptomatik konnte bisher nicht in ausreichendem Maße gelindert werden. Die Patientin ist dadurch offensichtlich stark belastet.

Die Patientin ist einwilligungsfähig und wünscht aufgrund der refraktären psychischen und existenziellen Symptomatik eine kontinuierliche und tiefe Sedierung.

Bitte geben Sie für jede Aussage an, inwieweit Sie dieser zustimmen.

Der Wunsch der Patientin ist für mich nachvollziehbar.

Die Patientin hat ein Recht darauf, dass ihrem Wunsch entsprochen wird.

Ich würde dem Wunsch nur nachkommen, wenn vorherige Versuche einer flachen bzw. intermittierenden Sedierung keine ausreichende Symptomlinderung erzielen konnten.

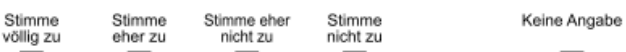
Ich würde dem Wunsch nur nachkommen, wenn sich die Patientin bereits unmittelbar in der Sterbephase befindet.

Ich würde generell keine kontinuierliche tiefe Sedierung durchführen.

Die Patientin ist einwilligungsfähig und wünscht aufgrund der refraktären psychischen und existenziellen Symptomatik eine kontinuierliche und tiefe Sedierung. Eine künstliche Ernährung während der Sedierung lehnt sie ausdrücklich ab. Bitte geben Sie für jede Aussage an, inwieweit Sie dieser zustimmen.

$\begin{aligned} & \text { Der Wunsch der Patientin ist für mich } \\ & \text { nachvollziehbar. }\end{aligned}$
$\begin{aligned} & \text { Die Patientin hat ein Recht darauf, dass } \\ & \text { ihrem Wunsch entsprochen wird. }\end{aligned}$

Eine künstliche Ernährung bei einer kontinuierlichen tiefen Sedierung wäre in meinen Augen medizinisch nicht indiziert.

Welches Vorgehen würden Sie im Kontext der zuletzt beschriebenen Situation als moralisch belastender empfinden?

$\square$ Abbruch einer bestehenden

künstlichen Ernährung über PEG

mit Beginn der Sedierung. $\square$ Ich würde beide Szenarien als gleich belastend empfinden. selbstständig essenden und trinkenden

Patientin und Verzicht auf künstliche Ernährung während der Sedierung. 
\begin{tabular}{l|l|l|l} 
EvaSys & Medizinethische Bewertung der Palliativen Sedierung bei ALS-Patientinnen und -Patienten & Electric Paper \\
\hline
\end{tabular}

\section{Fallbeispiel 3}

Sie behandeln einen ALS-Patienten im Endstadium der Erkrankung, der aufgrund fortschreitender respiratorischer Insuffizienz seit einigen Monaten invasiv (über eine Trachealkanüle) beatmet wird. Der Patient weist zudem eine starke körperliche Symptomatik mit fortgeschrittener Tetraparese, Dysphagie, Dysarthrie, Schmerzen durch Spastik und Muskelkrämpfe auf und ist dadurch offensichtlich stark belastet.

Der Patient ist einwilligungsfähig und wünscht nun eine Beendigung der invasiven Beatmung bei gleichzeitiger Palliativer Sedierung.

Bitte geben Sie für jede Aussage an, inwieweit Sie dieser zustimmen.

$\begin{aligned} & \text { Der Wunsch des Patienten ist für mich } \\ & \text { nachvollziehbar. }\end{aligned}$
$\begin{aligned} & \text { Der Patient hat ein Recht darauf, dass } \\ & \text { seinem Wunsch entsprochen wird. }\end{aligned}$

\section{Persönliche Daten}

Geschlecht:

$\square$ weiblich $\quad \square$ männlich $\square$ anderes / Keine Angabe
Alter:
$\square$ unter 35
$35-45$
$45-55$
$55-65$
$\square$ über 65
Welche Facharztausbildung(en) haben Sie absolviert?
$\square$ Neurologie $\square$ Anästhesiologie $\square$ Innere Medizin
$\square$ Sonstige

Haben Sie die Zusatz-Weiterbildung Palliativmedizin absolviert?

$\square \mathrm{Ja}$

$\square$ Nein

Haben Sie weitere Anmerkungen zum Thema oder zur Befragung? 


\subsection{Anschreiben}

\section{AEM \\ Akademie für Ethik \\ in der Medizin}

Geschäftsführung und Wiss. Leitung Prof. Dr. Alfred Simon

37099 Göttingen Briefpost Humboldtallee 36, 37073 Gouttingen Adresse 0551/ 39-9680 Telefon $0551 /$ 39-33996 Fax simon@aem-online.de E-Mail

24. August 2019 Datum

\section{- Befragung zur Palliativen Sedierung von ALS-Patientinnen und -Patienten}

Sehr geehrte Kollegin, sehr geehrter Kollege,

die Palliative Sedierung hat als Maßnahme zur Linderung extremer Belastungen in der palliativmedizinischen Praxis zunehmend an Bedeutung gewonnen. Es gibt jedoch in Deutschland bislang nur wenige wissenschaftliche Arbeiten, die sich mit dem Einsatz der Palliativen Sedierung bei neurologischen Patientinnen und Patienten beschäftigen.

Im Rahmen einer medizinischen Doktorarbeit führen wir eine bundesweite Befragung von weiterbildungsermächtigten Ärztinnen und Ärzten der Neurologie und der Palliativmedizin durch. Wir möchten herausfinden, inwieweit die Palliative Sedierung in der Behandlung der Amyotrophen Lateralsklerose zum Einsatz kommt und wie ein solcher Einsatz von den teilnehmenden Ärztinnen und Ärzten ethisch bewertet wird.

Auf Basis einer Zufallsstichprobe wurden Sie für die Teilnahme an unserer Befragung ausgewählt. Wir bitten Sie herzlich, an der Befragung teilzunehmen und den beiliegenden Fragebogen zusammen mit dem Deckblatt bis zum 24. Juni 2018 an die auf dem Deckblatt angegebene Adresse zurückzuschicken.

Für den Abgleich des Fragebogenrücklaufs ist die Angabe Ihres Namens und Ihrer Adresse auf dem Deckblatt notwendig (Stempel ausreichend). Wir garantieren eine anonymisierte Auswertung und vernichten das Deckblatt selbstverständlich vor der Dateneingabe.

Für die Bearbeitung des Fragebogens sollten Sie etwa 10 Minuten Zeitaufwand einplanen. Mit Ihrer Teilnahme leisten Sie einen wichtigen Beitrag zum Gelingen unseres Projekts. Wenn Sie an den Ergebnissen der Studie interessiert sind, schicken wir Ihnen diese gerne an die von Ihnen angegebene Adresse.

Für Rückfragen stehen wir Ihnen gerne zur Verfügung (laura.salzmann@stud.uni-goettingen.de).

Mit freundlichen Grüßen

Prof. Dr. Alfred Simon

Akademie für Ethik in der Medizin
Prof. Dr. Bernd Alt-Epping

Klinik für Palliativmedizin, UMG
Laura Salzmann

Doktorandin 


\subsection{Deckblatt}

\section{Befragung zur Palliativen Sedierung von ALS-Patientinnen und -Patienten}

Bitte schicken Sie den ausgefüllten Fragebogen zusammen mit diesem Deckblatt bis zum 24.06.2018 an folgende Adresse:

Akademie für Ethik in der Medizin

Universitätsmedizin Göttingen

Humboldtallee 36

37073 Göttingen

Deckblatt (wird vor der Dateneingabe vernichtet):

Name und Adresse des Absenders (Stempel):

Ich bin an den Ergebnissen der Studie interessiert. Bitte schicken Sie mir diese an die oben genannte Adresse.

$\square$ Ja $\quad \square$ Nein 


\title{
6.4 Recall-Fragebogen
}

\author{
AEM \\ Akademie für Ethik \\ in der Medizin
}

Universitâtsmedizin Göttingen, 37099 Göttingen

Akademie für Ethik in der Medizin, Humboldtallee 36, 37073 Göttingen

Geschäftsführung und Wiss. Leitung Prof. Dr. Alfred Simon

37099 Göttingen Briefpost Humboldtallee 36, 37073 Göttingen Adresse 0551 / 39-9680 Telefon 0551/ 39-33996 Fax simon@aem-online.de E-Mail

17. Mai 2018 Datum

\section{- Befragung zur Palliativen Sedierung von ALS-Patientinnen und -Patienten}

Sehr geehrte Kollegin, sehr geehrter Kollege,

vor einigen Wochen hatten wir Ihnen einen Fragebogen zum Thema Palliative Sedierung von ALSPatientinnen und Patienten zugeschickt. Wir hatten Sie gebeten, auf dem Deckblatt Ihre Adresse anzugeben, damit wir nachvollziehen können, ob Ihr Fragebogen bereits eingegangen ist. Das Deckblatt wird vor der Auswertung entfernt und vernichtet.

Nach unseren Unterlagen haben wir von Ihnen noch keine Antwort erhalten.

Da es sich um eine repräsentative Befragung handelt, ist es sehr wichtig, dass möglichst viele Teilnehmerinnen und Teilnehmer antworten. Wir bitten Sie daher nochmals herzlich, den Fragebogen zu beantworten und bis zum 19. August 2018 an uns zurückzusenden.

Sollten Sie den Fragebogen verlegt haben, senden wir Ihnen gerne einen neuen zu. Bitte geben Sie uns eine kurze Rückmeldung (E-Mail: laura.salzmann@stud.uni-goettingen.de).

Wenn Sie den Fragebogen aus bestimmten Gründen nicht beantworten können, bitten wir Sie, uns diese mit Hilfe des beiliegenden Antwortbogens mitzuteilen. Wir benötigen die Angaben, um die Studie angemessen auswerten zu können.

Einige Teilnehmerinnen und Teilnehmer haben uns den Fragebogen ohne Angabe der Adresse auf dem Deckblatt zurückgesendet. Sollte dies auf Sie zutreffen, betrachten Sie dieses Schreiben bitte als gegenstandslos.

Für Ihre Teilnahme bedanken wir uns noch einmal sehr herzlich!

Prof. Dr. Alfred Simon Akademie für Ethik in der Medizin
Prof. Dr. Bernd Alt-Epping

Klinik für Palliativmedizin, UMG
Laura Salzmann

Doktorandin 


\section{Befragung zur Palliativen Sedierung von ALS-Patientinnen und -Patienten}

Bitte senden Sie diesen Antwortbogen bis zum 19. August 2018 an folgende Adresse:

Akademie für Ethik in der Medizin

Universitätsmedizin Göttingen

Humboldtallee 36

37073 Göttingen

Fax: $0551 / 39-33996$

Gründe für die Nicht-Teilnahme an der Befragung:

- Mir ist die Beantwortung aus zeitlichen Gründen nicht möglich.

- Ich habe datenschutzrechtliche Bedenken gegen die Beantwortung des Fragebogens.

- Ich habe moralische Bedenken hinsichtlich der Beantwortung des Fragebogens/der Studie.

a Ich habe andere Gründe, und zwar: 


\subsection{Freitextantworten}

Haben Sie bereits eine Palliative Sedierung bei einer ALS-Patientin bzw. einem ALSPatienten durchgeführt? (Ja/Nein)

Wenn ja, bei welchen therapierefraktären Symptomen?

1. Dysphagie, Ablehnung der Trachealkanüle [F14184U1068412506P1PL1015V1]

2. Luftnot, Angst bes. Erstickungsangst [F14184U1068412506P1PL147V1]

3. Dyspnoe, Angst, Schmerz [F14184U1068412506P1PL181V1]

4. Ausgeprägte Parese mit Spastik, Dysphagie, Dysarthrie [F14184U1068412506P1PL225V1]

5. Dyspnoe/Schmerzen durch Spastik, Schluckstörungen/Dysphagie [F14184U1068412506P1PL385V1]

6. Patientenwunsch nach Schlaf, Ruhe [F14184U1068412506P1PL566V1]

7. Dyspnoe, Schmerzen, therapierefraktärer Husten(reiz) bei unzureichendem Hustenstoß, Verschleimung, maximale Schwäche; Erstickungsanfälle [F14184U1068412506P1PL574V1]

8. Schluck $+[\ldots]$-störung, intermitt. Atemstörung mit Problemen d. Abhustens [F14184U1068412506P1PL856V1]

9. Progrediente Ateminsuffizienz, Pat. hatte eine Beatmung abgelehnt [F14184U1068412506P1PL105V1]

10. Angst, Atemnot [F14184U1068412506P1PL295V1]

11. Luftnot, Erstickungsgefühl [F14184U1068412506P1PL568V1]

12. Dyspnoe [F14184U1068412506P1PL876V1]

13. Atemnot, Pneumonie, respiratorische Insuffizienz [F14184U1068412506P1PL894V1]

14. Atemnot [F14184U1068412506P1PL449V1]

15. Schluckstörung, respiratorische Insuffizienz, Tetraplegie [F14184U1068412506P1PL501V1]

16. Dyspnoe, Angst [F14184U1068412506P1PL549V1]

17. Dyspnoe, Pneumonie, respiratorisches Versagen [F14184U1068412506P1PL627V1]

18. Dyspnoe, Erstickungsangst [F14184U1068412506P1PL725V1]

19. Atemnot/Dyspnoe [F14184U1068412506P1PL786V1]

20. Massive Dyspnoe, Dysphagie, Immobilität [F14184U1068412506P1PL979V1]

21. Anhaltende Beatmungspflicht, nach Verschlechterung i.R. einer Pneumonie war die Beatmungsentwöhnung nicht möglich, Leben mit Beatmung vom Pat. abgelehnt [F14184U1068412506P1PL88V1]

22. Dyspnoe [F14184U1068412506P1PL100V1]

23. Atemnot [F14184U1068412506P1PL381V1]

24. Terminale Sedierung ist in unserem Hause Bestandteil der Palliativstation

[F14184U1068412506P1PL423V1] 
25. Progrediente, anders nicht kontrollierbare Dyspnoe, Beendigung einer Beatmung [F14184U1068412506P1PL630V1]

26. Dyspnoe, die anderweitig nicht kontrollierbar war [F14184U1068412506P1PL880V1]

27. Intermittierende schwere Atemnotattacken [F14184U1068412506P1PL918V1]

28. Dysphagie, zunehmende Dyspnoe, Schmerzen bei vollständiger Immobilität und [...] [F14184U1068412506P1PL141V1]

29. Angst und Agitation im Zusammenhang mit Ateminsuffizienz [F14184U1068412506P1PL153V1] 30. Dyspnoe [F14184U1068412506P1PL232V1]

31. Dyspnoe, Angst, Schmerzen, Hypersalivation [F14184U1068412506P1PL362V1]

32. Dyspnoe, Schwäche, Schmerzen [F14184U1068412506P1PL475V1]

33. Dyspnoe, Angst [F14184U1068412506P1PL647V1]

34. Präfinalphase mit resp. Insuffizienz [F14184U1068412506P1PL726V1]

35. Quälende [...] Symptome, aber auch Tetraparese mit Atemstörung [F14184U1068412506P1PL791V1]

36. Lebensbedrohliche Dyspnoe [F14184U1068412506P1PL189V1]

37. Dyspnoe, Dysphagie, Aspiration, Angst/Panik, Schmerzen, [...] [F14184U1068412506P1PL419V1]

38. Unerträgliche Luftnot, Pat. hat Maskenbeatmung / Respiratortherapie abgelehnt [F14184U1068412506P1PL456V1]

39. Dyspnoe [F14184U1068412506P1PL588V1]

40. Dyspnoe, Angst, Unruhe [F14184U1068412506P1PL624V1]

41. Luftnot und Verzweiflung [F14184U1068412506P1PL637V1]

42. Dyspnoe bei Ateminsuffizienz [F14184U1068412506P1PL714V1]

43. Atemnot, Panik [F14184U1068412506P1PL372V1]

44. Atemnot [F14184U1068412506P1PL378V1]

45. Atemnot, Angst, körperliche Symptomatik [F14184U1068412506P1PL584V1]

46. Angst, Unruhe, Dyspnoe [F14184U1068412506P1PL620V1]

47. Atemnot, Angstsymptomatik akut, Unruhe [F14184U1068412506P1PL659V1]

48. Ateminsuffizienz, Schluckstörung [F14184U1068412506P1PL785V1]

49. Nicht bei ALS Pat., aber bei anderen schon [F14184U1068412506P1PL871V1]

50. Stärker werdende Atemnot! [F14184U1068412506P1PL874V1]

51. Dyspnoe, Schmerzen durch Spastik therapierefraktär [F14184U1068412506P1PL961V1]

52. Luftnot, Schmerzen, Depression [F14184U1068412506P1PL980V1]

53. Angst, nicht beherrschbare Luftnot [F14184U1068412506P1PL989V1]

54. Ateminsuffizienz bei Ablehnung einer maschinellen Beatmung [F14184U1068412506P1PL70V1] 
55. Dyspnoe, Dysphagie, Dysarthrie, Spastik und Muskelkrämpfe [F14184U1068412506P1PL190V1]

56. Atemnot + Verweigerung beatmet zu werden [F14184U1068412506P1PL266V1]

57. Nahezu vollständige Lähmung der Muskulatur einschließlich Atemmuskulatur, Dyspnoe, Angst [F14184U1068412506P1PL273V1]

58. Im stat. Hospiz - Luftnot [F14184U1068412506P1PL331V1]

59. Atemnot, Delir, Angst [F14184U1068412506P1PL366V1]

60. Wunsch zu sterben, Ø Schmerzen oder Krämpfe [F14184U1068412506P1PL382V1]

61. Atemnot [F14184U1068412506P1PL462V1]

62. Luftnot [F14184U1068412506P1PL528V1]

63. Dyspnoe, komplexe Symptomlast aus den klass. ALS-Symptomen mit und ohne Beatmung, terminales Weaning [F14184U1068412506P1PL575V1]

64. Atemnot [F14184U1068412506P1PL580V1]

65. Parese der Extremitäten, Parese der Thorax-[...], Schluckbeschwerden, Pat. will nicht beatmet werden, Dysphagie, Dysarthrie usw. [F14184U1068412506P1PL631V1]

66. Angst, Dysphagie, begleitend zu freiwilligem Verzicht auf Flüssigkeit \& Nahrung [F14184U1068412506P1PL651V1]

67. Dyspnoe, Angst, Schmerzen [F14184U1068412506P1PL661V1]

68. Dyspnoe, Angst, Panik, Spasmen [F14184U1068412506P1PL915V1]

69. Angst, Luftnot, (Schmerzen) [F14184U1068412506P1PL940V1]

70. Atemnot, Schmerz [F14184U1068412506P1PL68V1]

71. Angst, Atemnot [F14184U1068412506P1PL270V1]

72. Atemnot u. Schluckstörungen [F14184U1068412506P1PL652V1]

73. Atemnot [F14184U1068412506P1PL715V1]

74. 1.) Bei organ. Psychosen als Folge der ALS 2.) Bei Angststörung aufgrund von Atemnot 3.) Bei weiteren neurol. Komplikationen wie Epilepsie 4.) Bei schwerer affektiver Begleitsymptomatik u. Suizidalität [F14184U1068412506P1PL813V1]

75. Dyspnoe, Beendigung einer NIV-Therapie [F14184U1068412506P1PL883V1]

76. Dyspnoe, Unruhe [F14184U1068412506P1PL904V1]

77. Existential distress und Ablehnung einer (weiteren) Beatmungstherapie

[F14184U1068412506P1PL1037V1]

78. Schmerz, psychomot. Unruhe, Insomnie [F14184U1068412506P1PL1039V1]

79. Schwere Angst und Panikstörung bei kontinuierlicher CPAP-Beatmung, Spastik [F14184U1068412506P1PL156V1]

80. Massive Dyspnoe in der Sterbephase [F14184U1068412506P1PL286V1]

81. Atemnot [F14184U1068412506P1PL309V1] 
82. Therapierefraktäre Atemnot + Angst [F14184U1068412506P1PL341V1]

83. Atemnot, Angst, Unruhe [F14184U1068412506P1PL377V1]

84. Progredientes Versagen der Atemmuskulatur, Hypersalivation u. Dysphagie + zunehmende Atemlähmung [F14184U1068412506P1PL468V1]

85. Dyspnoe [F14184U1068412506P1PL552V1]

86. Luftnot, Schmerzen, existentielles Leid [F14184U1068412506P1PL555V1]

87. Tetraparese, Dysphagie, Atemnot [F14184U1068412506P1PL824V1]

88. Dyspnoe [F14184U1068412506P1PL841V1]

89. Präfinale Unruhe/Luftnot [F14184U1068412506P1PL870V1]

90. Schmerzen, Angst, Dyspnoe [F14184U1068412506P1PL884V1]

91. Dyspnoe/Angst [F14184U1068412506P1PL26V1]

92. [...] Luftnot; psychische Dekompensation bei extremer Hilfsbedürftigkeit; rezid. Aspirationen [F14184U1068412506P1PL183V1]

93. Nicht sprechen, nicht schlucken, nicht selbstständig atmen [F14184U1068412506P1PL327V1]

94. Zunehmende Atemnot, Pat. wünschte keine invasive Beatmung

[F14184U1068412506P1PL928V1]

95. Angst [F14184U1068412506P1PL1020V1]

96. Bei Absetzen des Beatmungsgerätes, auf Pat. Wunsch [F14184U1068412506P1PL85V1]

97. Erstickungsanfälle, die nicht ausreichend (subjektiv für den Patienten) auf die sonstige Therapie reagieren. Häufiger: Seelische Not und Verzweiflung!

98. Dyspnoe [F14184U1068412506P1PL723V1]

99. Atemnot [F14184U1068412506P1PL763V1]

100. Dyspnoe [F14184U1068412506P1PL854V1]

101. Atemnot, Schmerzen, Spastik [F14184U1068412506P1PL24V1]

102. Luftnot, Angst [F14184U1068412506P1PL135V1]

103. Angst, Luftnot [F14184U1068412506P1PL271V1]

104. Dyspnoe, Angst, Panik, psychoexistenzielles Leiden, Schmerzen [F14184U1068412506P1PL274V1]

105. Atemnot, quälender Husten, bronchiale Verschleimung, Angst, Dysphagie, existenzielles Leid [F14184U1068412506P1PL361V1]

106. Unruhe, Atemnot [F14184U1068412506P1PL368V1]

107. Luftnot trotz laufender Beatmung, daraus resultierende Angst [F14184U1068412506P1PL444V1]

108. Angst [F14184U1068412506P1PL551V1]

109. [...] Dyspnoe [F14184U1068412506P1PL775V1]

110. Ateminsuffizienz [F14184U1068412506P1PL13V1] 


\begin{tabular}{|l|}
\hline 111. Luftnot [F14184U1068412506P1PL138V1] \\
\hline 112. Unerträgliches Leid, Schwäche, Ateminsuffizienz [F14184U1068412506P1PL384V1] \\
\hline 113. Atemnot [F14184U1068412506P1PL396V1] \\
\hline 114. Dyspnoe [F14184U1068412506P1PL467V1] \\
\hline 115. Angst [F14184U1068412506P1PL530V1] \\
\hline 116. Dyspnoe bei fortschreitender Parese [F14184U1068412506P1PL578V1] \\
\hline 117. Dyspnoe, Angst [F14184U1068412506P1PL25V1] \\
\hline 118. Atemnot, Angst, Erstickungsangst, Panik, Aspiration [F14184U1068412506P1PL146V1] \\
\hline 119. Luftnot, Unruhe, Delir, Schluckstörungen, Aspiration [F14184U1068412506P1PL178V1] \\
\hline 120. Atemnot, existentielles Leid [F14184U1068412506P1PL379V1] \\
\hline 121. Dyspnoe, Schmerzen [F14184U1068412506P1PL563V1] \\
\hline 122. Dyspnoe, Spastik [F14184U1068412506P1PL1025V1] \\
\hline 123. Dyspnoe, Angst, Schmerzen [F14184U1068412506P1PL1026V1] \\
\hline
\end{tabular}


Kategorisierung der oben aufgeführten Freitextantworten

\begin{tabular}{|c|c|c|c|c|c|}
\hline Begriffsfeld & Luftnot & Ateminsuffizienz & Dysphagie & Parese & Angst \\
\hline $\mathbf{N}$ & 95 & 16 & 18 & 9 & 39 \\
\hline $\begin{array}{l}\text { Genannte Be- } \\
\text { griffe }\end{array}$ & $\begin{array}{l}\text { - Luftnot } \\
\text { - Dyspnoe } \\
\text { - Atemnot } \\
\text { - Erstickungsanfälle } \\
\text { - Atemnotattacken }\end{array}$ & $\begin{array}{l}\text { - Ateminsuffizienz } \\
\text { - Atemstörung } \\
\text { - Respiratorische } \\
\text { Insuffizienz } \\
\text { - Respiratorisches } \\
\text { Versagen } \\
\text { - Atemlähmung } \\
\text { - Lähmung der } \\
\text { Atemmuskulatur } \\
\text { - Nicht selbstständig } \\
\text { atmen } \\
\text { - Progredientes Versagen } \\
\text { der Atemmuskulatur } \\
\text { - Lähmung der } \\
\text { Muskulatur } \\
\text { einschließlich } \\
\text { Atemmuskulatur }\end{array}$ & $\begin{array}{l}\text { - Dysphagie } \\
\text { - Schluckstörungen } \\
\text { - Nicht schlucken } \\
\text { - Schluckbeschwerden }\end{array}$ & $\begin{array}{l}\text { - Parese } \\
\text { - Tetraparese } \\
\text { - Parese der Extremitäten, Parese der } \\
\text { Thorax[...] } \\
\text { - Lähmung der Muskulatur } \\
\text { - Vollständige Immobilität } \\
\text { - Immobilität } \\
\text { - Tetraplegie }\end{array}$ & $\begin{array}{l}\text { - Angst } \\
\text { - Schwere Angst } \\
\text { - Panik } \\
\text { - Panikstörung } \\
\text { - Angststörung } \\
\text { - Angstsymptomatik } \\
\text { - Erstickungsangst }\end{array}$ \\
\hline
\end{tabular}




\begin{tabular}{|c|c|c|c|c|c|}
\hline Begriffsfeld & Ablehnung / Absetzen von Beatmung & Unruhe & Schmerz & Spastik & Sonstige \\
\hline $\mathbf{N}$ & 13 & 10 & 19 & 8 & 38 \\
\hline $\begin{array}{l}\text { Konkrete } \\
\text { Begriffe }\end{array}$ & $\begin{array}{l}\text { - Patient wünscht keine invasive Beatmung } \\
\text { - Bei Absetzen des Beatmungsgeräts } \\
\text { - Ablehnung einer (weiteren) } \\
\text { Beatmungstherapie } \\
\text { - Beendigung einer NIV-Therapie } \\
\text { - Patient will nicht beatmet werden } \\
\text { - Terminales Weaning } \\
\text { - Verweigerung beatmet zu werden } \\
\text { - Ablehnung einer maschinellen Beatmung } \\
\text { - Patient hat Maskenbeatmung / } \\
\text { - Respiratortherapie abgelehnt } \\
\text { - Beendigung einer Beatmung } \\
\text { - Leben mit Beatmung vom Pat. abgelehnt } \\
\text { - Ablehnung der Trachealkanüle }\end{array}$ & $\begin{array}{l}\text { - Unruhe } \\
\text { - Agitation }\end{array}$ & - Schmerzen & $\begin{array}{l}\text { - Spastik } \\
\text { - Spasmen }\end{array}$ & $\begin{array}{l}\text { - Aspiration } \\
\text { - Existenzielles Leid } \\
\text { - Verzweiflung } \\
\text { - Depression } \\
\text { - Seelische Not } \\
\text { - Psychische Dekompensation bei extremer } \\
\text { Hilfsbedürftigkeit } \\
\text { - Delir } \\
\text { - Schwäche } \\
\text { - Pneumonie } \\
\text { - Insomnie } \\
\text { - Hypersalivation } \\
\text { - Muskelkrämpfe } \\
\text { - Epilepsie } \\
\text { - Bei schwerer affektiver Begleitsymptomatik } \\
\text { - Unerträgliches Leid } \\
\text { - Psychoexistenzielles Leiden } \\
\text { - Auf Patientenwunsch } \\
\text { - Existential Distress } \\
\text { - Suizidalität } \\
\text { - Organische Psychosen } \\
\text { - Begleitend zu freiwilligem Verzicht auf } \\
\text { - Flüssigkeit und Nahrung } \\
\text { - Wunsch zu sterben } \\
\text { - Verschleimung } \\
\text { - Patientenwunsch nach Schlaf, Ruhe } \\
\text { - Dysarthrie } \\
\text { - Nicht sprechen }\end{array}$ \\
\hline
\end{tabular}




\section{Haben Sie weitere Anmerkungen zum Thema oder zur Befragung?}

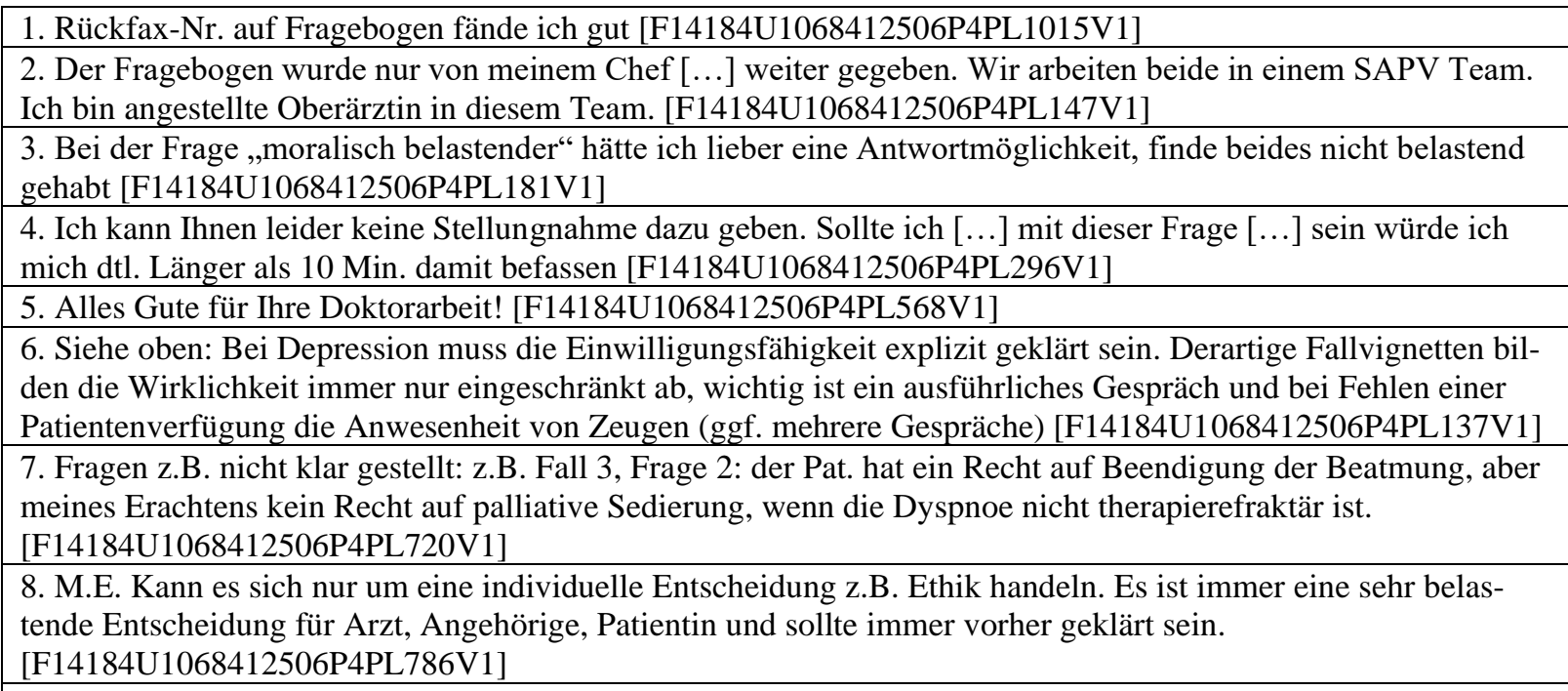

9. Der Verzicht auf eine Sondenernährung während der Sedierung ist für mich nicht belastend, daher finde ich die jeweils letzte Frage FB1+2 nicht günstig formuliert. Hierbei entscheidend ist für mich die Sedierung einer bisher noch relativ [...] Patientin, die isst + trinkt. Dies wäre belastender, [...] und dann wäre der Patientenwille verbindlich [F14184U1068412506P4PL88V1]

10. Falls Indikation zu tiefer Sedierung gegeben ist, ist m.E. eine Ernährung nicht mehr indiziert. Einsatz von Sedativa? [F14184U1068412506P4PL381V1]

11. Keine Erfahrung mit ALS-Pat [F14184U1068412506P4PL903V1]

12. Sehr komplexes Thema. Wir haben ein Vorgehen für unsere Klinik erarbeitet, in der wir die palliative und die terminale Sedierung klar unterscheiden und eine unterschiedliche Vorgehensweise festgelegt haben. Mit vielen Grüßen! [F14184U1068412506P4PL918V1]

13.Eine Palliativsedierung behandelt nicht Angst, Panik, Depression (siehe Fallbeispiel 2)

[F14184U1068412506P4PL933V1]

14. Wichtiges Thema [F14184U1068412506P4PL49V1]

15. Ich finde solche Befragungen zwar wichtig aber auch sehr schwierig, weil die jeweils individuelle Situation und auch das Vertrauen und die Beziehung zum Behandlungsteam sowie die familiäre Situation müssen ausreichend berücksichtigt werden. [F14184U1068412506P4PL141V1]

16. Patientenverfügung ist nur ein Weg den Willen zu äußern + zu dokumentieren

[F14184U1068412506P4PL303V1]

17. Im Vordergrund stehen Achtung der [...] Lebensqualität, was die Achtung vor Autonomie, der [Würde?] und des möglichen Wohlbefindens des Pat. u. Umfeldes berücksichtigt [F14184U1068412506P4PL362V1]

18. Leider finde ich die Problematik zu komplex, um anhand der kurzen Vignetten [...] Aussagen machen zu können. Solche Entscheidungen sind [...] vielschichtig u. kaum als Fragebogen zu klären.

[F14184U1068412506P4PL430V1]

19. Der Versuch der Standardisierung der Antworten ist differenziert angelegt, wird aus meiner Sicht trotzdem nicht der komplexen klinischen Symptomatik gerecht. [...] ist eine gewisse Verallgemeinerung zwingend. Trotzdem guter Ansatz! [F14184U1068412506P4PL726V1]

20. Bei „künstlicher Ernährung“ sollte zwischen Volumen/Flüssigkeitsgabe \& Kalorienzufuhr differenziert werden [F14184U1068412506P4PL791V1]

21. Die Formulierung „die bisher nicht..." ist unscharf. Entscheidend ist, dass trotz Einsatz aller verfügbaren Maßnahmen kein Erfolg erzielt wurde [F14184U1068412506P4PL65V1]

22. Nach wie vor interessante Thematik, die insbesondere außerhalb der Neurologie zu wenig beachtet wird, auch bei anderen internistischen oder onkolog. Krankheitsbildern. Eigene Publikationen zum Thema ALS-Terminalphase: Palliative care and circumstances of dying in German ALS patients using non-invasive ventilation (ALS 2008). Diagnosis and treatment of bulbar symptoms in ALS. [F14184U1068412506P4PL419V1]

23. Vertrag mit dem aufklärungsfähigen Patienten oder Ethikkommision / Betreuungsgericht bzw. Verfügung / Vollmacht [F14184U1068412506P4PL716V1]

24. Ich behandele ausschließlich onkologische Pat. und bin daher für die Befragung nicht geeignet!

[F14184U1068412506P4PL724V1]

25. M.E. gibt es keine gültige [Information der] palliativen Sedierung [F14184U1068412506P4PL846V1] 


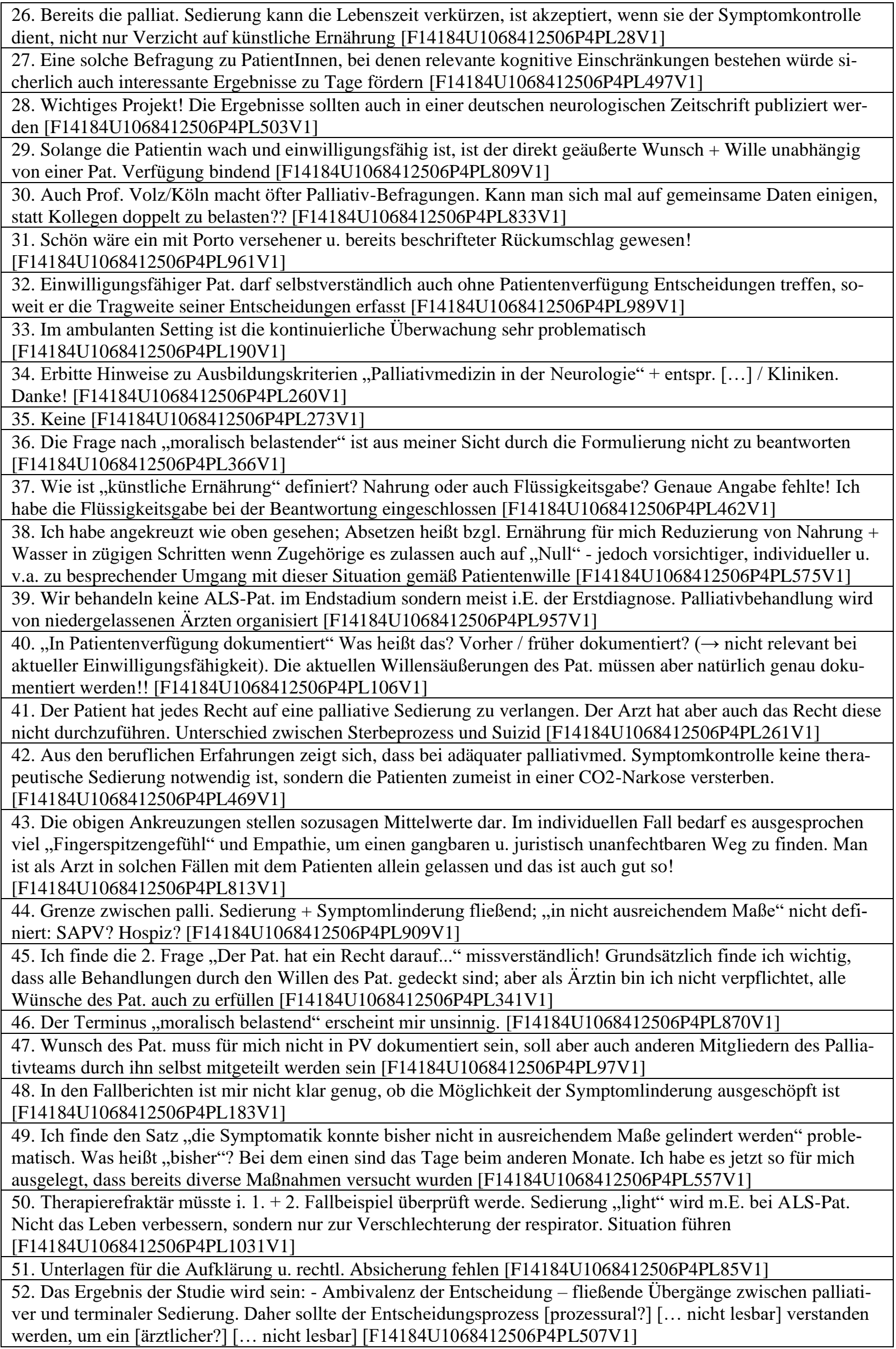


53. Mein Arbeitsfeld ist die pädiatrische Palliativversorgung, daher keine Erfahrung mit ALS-Patienten [F14184U1068412506P4PL882V1]

54. Gespräche und Dokumentation im Vorfeld mit allen Beteiligten und mind. 2-3 x im Abstand von X Tagen. Ggf. Supervision od. eth. Fallbesprechung für Konsens aller Beteiligten [F14184U1068412506P4PL274V1] 55. Nein, viel Glück mit der Studie, bin gespannt auf die Ergebnisse [F14184U1068412506P4PL361V1] 56. Leider waren die Antwortmöglichkeiten beschränkt / gekoppelt. Ich würde eine intensivierte Symptomkontrolle anbieten, die de facto in eine Sedierung mündet. Das Ziel ist ein anderes, das Vorgehen entspricht der erwähnten flachen Sedierung. Für eine dauerhafte tiefe Sedierung müsste ich eine stationäre Einrichtung finden, führe ich nicht [... nicht lesbar] [F14184U1068412506P4PL380V1]

57. Jede medizinische Behandlung bedarf der Einwilligung (Körperverletzung mit Duldung). Entzug der Einwilligung bedeutet bei Fortsetzung der Behandlung Körperverletzung (ohne Duldung). Verpflichtung zur Symptomlinderung besteht. Intensive Kommunikation u. Dokumentation [F14184U1068412506P4PL444V1]

58. Ich bin kein Jurist, daher mit den Tatbestandsmerkmalen der „Tötung auf V. „, nicht vertraut [F14184U1068412506P4PL530V1]

59. Die Fragen entsprechen nicht unserem Vorgehen. Gespräch mehrfach (2-3x) mit Zeugen + [Doku?] fehlt mir. Recht auf Sedierung: Pat. hat kein Anspruchsrecht auf [... nicht lesbar], dieses ist aber andererseits aufgrund der Symptomlast geboten [F14184U1068412506P4PL379V1]

60. Die Fragestellung der Sedierung eines ALS-Pat. der noch selbstständig essen + trinken kann, halte ich / wir nicht für eine Indikation über pall. Sedierung nachzudenken! Wenn, wie im Fall 3 der Wunsch nach Beendigung der Beatmung geäußert wird, gilt es dringend zu [beweisen], warum der Wunsch besteht! Auszuschließen ist z.B. ob der Pat. bei schlechter Pflege [... nicht lesbar] [F14184U1068412506P4PL461V1]

61. Beantwortung der Fragen sehr schwierig, weil je nach Pat. (Gesamtkonstellation) würde ich ggf. anders entscheiden. Entscheidung würde ich nicht alleine treffen - Mitbehandlung durch Palliativmediziner wäre in meinen Augen erforderlich [F14184U1068412506P4PL540V1]

Recall-Fragebogen: Gründe für die Nicht-Teilnahme an der Befragung:

1. Ich betreue keine ALS Patienten palliativ.

2. Leider konnte ich kaum Fragen beantworten, da ich die Fragestellungen in meiner Tätigkeit nicht / extrem selten habe. „Fehlende Kompetenz“ Fachärztin Gynäkologie mit Schwerpunkttätigkeit [nicht lesbar] $\rightarrow$ vereinzelt Mitbetreuung palliativer Patientinnen im Gyn-Bereich. ALS-Patienten hatte ich nie.

3. Ich betreue keine ALS-Patienten.

4. Bisher nicht durchgeführt.

5. Keine ALS-Pat. in Betreuung. Keine Erfahrung zu diesem Thema.

6. Prof. [aus Datenschutzgründen wird der Name nicht genannt] kümmert sich an unserer Klinik um die ALS Patienten hauptsächlich.

7. Wir führen keine Sedierung und keine Palliativbehandlung bei ALS durch.

8. Hatten seit längerer Zeit keine Pat. mit ALS, insgesamt kommt diese Patientengruppe nur sehr selten bei uns zur Aufnahme.

9. Wir hatten in den letzten Jahren nur ALS-Patienten zur Erstdiagnose - keine Patienten [...] der Sedierungssituation.

10. ALS-Pat. Sind bei uns extrem selten! $\rightarrow$ keine Erfahrung

11. Keine Patienten.

12. Keine klinische Erfahrung - da keine Palliativversorgung in der Geriatrischen Reha stattfindet.

13. Ich behandele keine ALS-Patienten.

14. Ich beschäftige mich mit den Patienten, die ALS haben, ich habe aber wenig Erfahrung dabei.

15. Keine Erfahrung bei palliativer Sedierung von ALS-Pt., da ambulant tätig.

16. Geringe ALS Pat. Zahl. 
17. Wir betreuen in unserer Abt. keine ALS-Patienten.

18. Ich habe zu wenig ALS-Patienten in der späteren Krankheitsphase, alle kommen von weit her und kleben dann nur am Hausarzt, der Rilutek weiter verschreibt.

19. Ich behandele keine ALS-Patienten in meiner Abteilung.

20. In unserer Abteilung für medizinische / neurologische Frührehabilitation habe ich keine ausreichende Erfahrung mit der palliativen Sedierung von ALS-Patienten.

21. Keine ALS Pat. da [keine] Neurologie $\rightarrow$ ALS an [... nicht lesbar]

22. Wir behandeln in unserer Reha-Klinik nur sehr selten ALS-Patienten, eine palliative Sedierung führen wir hier nie durch.

23. Wir haben keine Patienten mit o.g. Beschwerden.

24. Kein entsprechendes Pat.-Klientel.

25. Keine Erfahrungen mit Sedierung von ALS Patienten (2 ALS Patienten in 25 Jahren)

26. nicht interessiert

27. Betreue keine entsprechende Pat.

28. Dieses sensible Thema kann nicht mittels Fragebögen behandelt werden. Statistische Daten hierüber halte ich nicht für repräsentativ und nicht für sinnvoll.

29. Als Kinderarzt behandele ich keine Patienten mit ALS.

30. Wir behandeln keine ALS-Patienten.

31. Bei fehlendem neurologischem Schwerpunkt in unserem Haus wurde im 8-jährigen Bestehen der Palliativstation keine einzige pall. Sedierung bei ALS-Pat. Durchgeführt. Generell wurden in dieser Zeit unter 10 ALS-Pat. überhaupt hier behandelt.

32. Wir behandeln keine ALS-Pat.

33. Liebe Kolleginnen, Kollegen, die palliative Betreuung der ALS-Pat. in dieser Region wird von Hausärzten und / oder palliativen Einrichtungen vorgenommen.

34. Seit 1.7.18 haben wir keine Neurologie mehr.

35. Zu wenig Erfahrung mit ALS-Patienten.

36. Ich bin seit mehreren Jahren nicht an der Versorgung von Palliativpatienten beteiligt, da mein Aufgabengebiet sich fast ausschließlich auf den operativ anästhesiologischen Bereich beschränkt.

37. Ich arbeite im Rahmen einer GP für Neurol. u. Psychiatrie nahezu ausschließlich als Neuroradiologe und habe seit mehr als 25 Jahren keine Patienten mit ALS u. ähnlichen neurodegenerativen Erkrankungen behandelt oder betreut. Fühle mich daher nicht ausreichend Kompetenz zum Thema! Würde in meinem betreuten Patientengut (Hirntumorpatienten) solche Entscheidungen immer in Abhängigkeit von der gegebenen Situation als Einzelfallentscheidung für und - wenn möglich - mit dem Patienten sehen.

38. Palliative Sedierung bei uns nur bei Krebspatienten bisher. Keine Erfahrung mit Pat. mit ALS.

39. Haben bei uns keine ALS Patienten behandelt, da keine Neurologie bei uns im Haus.

40. Ich arbeite in einer geriatr. Rehaklinik, in der wir sehr selten ALS-Patienten aufnehmen. Wenn ALS-Patienten kommen, befinden sie sich meist im Anfangsstadium der ALS. Sie gelten noch als rehafähig. Eine Palliativsituation mit ALS-Patienten habe ich in der Rehaklinik noch nicht erlebt.

41. Mag nicht.

42. Schwierige Fragestellung, 2 Fälle in der Familie $\rightarrow$ fällt mir schwer. 


\section{$7 \quad$ Literaturverzeichnis}

Alt-Epping B, Nauck F (2012): Der Wunsch des Patienten - ein eigenständiger normativer Faktor in der klinischen Therapieentscheidung? Ethik Med 24, 19-28

Alt-Epping B, Geyer A, Nauck F (2008): Palliativmedizinische Konzepte bei nicht-onkologischen Grunderkrankungen. DMW - Dtsch Med Wochenschr 133, 1745-1749

Alt-Epping B, Sitte T, Nauck F, Radbruch L (2010): Sedierung in der Palliativmedizin - Leitlinie für den Einsatz sedierender Maßnahmen in der Palliativversorgung. Z Palliativmed 11, $112-122$

Andersen PM, Abrahams S, Borasio GD, de Carvalho M, Chio A, Damme PV, Hardiman O, Kollewe K, Morrison KE, Petri S et al. (2012): EFNS guidelines on the clinical management of amyotrophic lateral sclerosis (MALS) - revised report of an EFNS task force. Eur J Neurol 19, 360-375

AWMF (2018): Erweiterte S3-Leitlinie Palliativmedizin für Patienten mit einer nicht-heilbaren Krebserkrankung. https://www.awmf.org/uploads/tx_szleitlinien/128-001OLl_S3_Palliativmedizin_2020-02.pdf; letzter Zugriff am 05.09.2020

Bausewein C (2005): Bislang unterschätzt. Palliativmedizin für Nicht-Tumorpatienten. Klin $\underline{34}$, $19-23$

Beauchamp TL, Childress JF: Principles of biomedical ethics. 5. Auflage; Oxford University Press, New York 2001

Beck D (2004): Ist terminale Sedierung medizinisch sinnvoll oder ersetzbar? Ethik Med 16, 334 341

Borasio GD, Voltz R (2000): Aufklärung und Palliativmedizin in der Neurologie am Beispiel der amyotrophen Lateralsklerose. Internist $\underline{41}, 627-632$

Borasio GD, Volkenandt M (2007): Palliativmedizin - weit mehr als nur Schmerztherapie. Gynäkol 40, 941-946

Bozzaro C (2015): Der Leidensbegriff im medizinischen Kontext: Ein Problemaufriss am Beispiel der tiefen palliativen Sedierung am Lebensende. Ethik Med 27, 93-106

Braun TC, Hagen NA, Clark T (2003): Development of a clinical practice guideline for palliative sedation. J Palliat Med $\underline{6}, 345-350$ 
Bundesverfassungsgericht 2020, https://www.bundesverfassungsgericht.de/SharedDocs/Entscheidungen/DE/2020/02/rs20200226_2bvr234715.html; letzter Zugriff am 22.04.2020

Cellarius V (2008): Terminal sedation and the „imminence condition“. J Med Ethics 34, 69-72

Chambaere K, Bilsen J, Cohen J, Rietjens JA, Onwuteaka-Philipsen BD, Mortier F, Deliens L (2010): Continuous deep sedation until death in Belgium: A nationwide survey. Arch Intern Med 170, 490-493

Cherny NI (2014): ESMO clinical practice guidelines for the management of refractory symptoms at the end of life and the use of palliative sedation. Annonc $\underline{25}, 143-152$

Cherny NI, Radbruch L, The Board of the European Association for Palliative Care (2009): European association for palliative care (EAPC) recommended framework for the use of sedation in palliative care. Palliat Med $\underline{23}, 581-593$

Connolly S, Galvin M, Hardiman O (2015): End-of-life management in patients with amyotrophic lateral sclerosis. Lancet Neurol $\underline{14}, 435-442$

Davis MP, Ford PA (2005): Palliative sedation definition, practice, outcomes, and ethics. J Palliat Med $\underline{8}, 699-701$

DGN (2015): Amyotrophe Lateralsklerose (Motoneuronerkrankungen). https://dgn.org/leitlinien/3012-11-18-11-amyotrophe-lateralsklerose-motoneuronerkrankungen; letzter Zugriff am 05.09.2020

DGN (2017): Edavarone: Eine neue Substanz für die Behandlung der ALS. https://dgn.org/neuronews/54-neuronews-2017/3436-edaravone-eine-neue-substanz-fuer-die-behandlungder-als; letzter Zugriff am 05.09.2020

Enck RE (1991): Drug-induced terminal sedation for symptom control. Am J Hosp Palliat Med $\underline{8}, 3-5$

Golla H, Ostgathe C, Gärtner J, Montag T, Düsterdiek A, Henseler-Plum I, Klein W, Wissmann G, Voltz R (2007): Neurologische Palliativmedizin - Inhalte und Organisationsformen. Aktuelle Neurol 34, 216-229

Hallenbeck JL (2000): Terminal sedation: Ethical implications in different situations. J Palliat Med 3, 313-320 
HRR-Strafrecht (2010): BGH 2 StR 454/09 - Urteil vom 25. Juni 2010 (LG Fulda), https://www.hrr-strafrecht.de/hrr/2/09/2-454-09.php?referer=db; letzter Zugriff am 05.09.2020

Husebø S, Mathis G: Was ist Palliativmedizin? Was ist Palliative Care? In: Husebø S, Mathis G (Hrsg.): Palliativmedizin. 6. Auflage; Springer, Berlin 2017, 1-10

Jaspers B, Nauck F, Lindena G, Elsner F, Ostgathe C, Radbruch L (2012): Palliative sedation in Germany: How much do we know? A prospective survey. J Palliat Med $\underline{15}, 672-680$

Jox RJ: Ethische Fragen bei neurodegenerativen Erkrankungen. In: Erbguth F, Jox J (Hrsg.): Angewandte Ethik in der Neuromedizin. Springer, Berlin 2017, 201-211

Kamprad M, Helm U (2018): Palliative Sedierung - trotz Leitlinien ein schwieriger Entscheidungsprozess. Dtsch Med Wochenschr 143, 574-581

Kreß H (2004): Selbstbestimmung am Lebensende: Die Bioethik-Kommission Rheinland-Pfalz zur Sterbehilfe und Sterbebegleitung. Ethik Med 16, 291-297

Kühnlein P, Kübler A, Raubold S, Worrell M, Kurt A, Gdynia H, Sperfeld A, Ludolph AC (2008): Palliative care and circumstances of dying in German ALS patients using non-invasive ventilation. Amyotroph Lateral Scler $\underline{9}, 91-98$

Leitlinie Amyotrophe Lateralsklerose: siehe DGN 2015

Leitlinie Palliativmedizin: siehe AWMF 2018

Lipuma SH (2013): Continuous sedation until death as physician-assisted suicide/euthanasia: A conceptual analysis. J Med Philos $\underline{38}$, 190-204

Ludolph AC: Motoneuronenerkrankungen. In: Berlit P (Hrsg.): Klinische Neurologie. 3. Auflage; Springer, Essen 2011, 597-618

Ludolph AC, Anneser J: Die Amyotrophe Lateralsklerose und andere Motoneuronenerkrankungen. In: Brandt T, Diener HC, Gerloff C (Hrsg.): Therapie und Verlauf neurologischer Erkrankungen. 6. Auflage; Kohlhammer, Stuttgart 2012, 1080-1090

Maessen M, Veldink JH, Onwuteaka-Philipsen BD, Hendricks HT, Schelhaas HJ, Grupstra HF, van der Wal G, van den Berg LH (2014): Euthanasia and physician-assisted suicide in amyotrophic lateral sclerosis: a prospective study. J Neurol 261, 1894-1901 
Maier BO, Sitte T: Grundlagen und Versorgungsstrukturen. In: Thöns M, Sitte S (Hrsg.): Repetitorium Palliativmedizin. 2. Auflage; Springer, Berlin 2016, 1-12

Marckmann G: Grundlagen ethischer Entscheidungsfindung in der Medizin. In: Marckmann G (Hrsg.): Praxisbuch Ethik in der Medizin. Medizinisch Wissenschaftliche Verlagsgesellschaft, Berlin 2015, 3-13

Materstvedt LJ, Clark D, Ellershaw J, Førde R, Gravgaard A-MB, Müller-Busch HC, Porta i Sales J, Rapin C-H (2003): Euthanasia and physician-assisted suicide: a view from an EAPC ethics task force. Palliat Med $\underline{17}, 97-101$

Meyer T, Dullinger JS, Münch C, Keil J-P, Hempel E, Rosseau S, Borisow N, Linke P (2008): Elektive Termination der Beatmungstherapie bei der amyotrophen Lateralsklerose. Nervenarzt $\underline{79}, 684-690$

Morita T, Tsunoda J, Inoue S, Chihara S (2001): Effects of high dose opioids and sedatives on survival in terminally ill cancer patients. J Pain Symptom Manage 21, 282-289

Müller-Busch HC (2004): „Terminale Sedierung“. Ausweg im Einzelfall, Mittelweg oder schiefe Ebene? Ethik Med 16, 369-377

Müller-Busch HC (2008): Kommentar I. Ethik Med 20, 135-137

Muller-Busch HC, Andres I, Jehser T (2003): Sedation in palliative care - a critical analysis of 7 years experience. BMC Palliat Care $\underline{2}(1), 2$

Müller-Busch HC, Oduncu FS, Woskanjan S, Klaschik E (2004): Attitudes on euthanasia, physician-assisted suicide and terminal sedation - A survey of the members of the German association for palliative medicine. Med Health Care Philos $\underline{7}, 333-339$

Nationaler Ethikrat (2006): Selbstbestimmung und Fürsorge am Lebensende. Stellungnahme. https://www.ethikrat.org/fileadmin/Publikationen/Stellungnahmen/Archiv/Stellungnahme_Selbstbestimmung_und_Fuersorge_am_Lebensende.pdf; letzter Zugriff am 05.09.2020

Neitzke G (2010): Zur Bedeutung von Leitlinien zum Umgang mit Sedierung am Lebensende. Schmerz $24,355-357$

Neitzke G, Frewer A (2004): Sedierung als Sterbehilfe? Zur medizinethischen Kultur am Lebensende. Ethik Med 16, 323-333 
Neitzke G, Oehmichen F, Schliep HJ, Wördehoff D (2010): Sedierung am Lebensende. Empfehlungen der AG Ethik am Lebensende in der Akademie für Ethik in der Medizin (AEM). Ethik Med 22, 139-147

Oechsle K, Radbruch L, Wolf C, Ostgathe C (2017): SOP - Palliative Sedierung. Onkol 233, 469475

Orentlicher D (1997): The supreme court and terminal sedation: Rejecting assisted suicide, embracing euthanasia. Hastings Const LAW Q 르, 947-968

Radbruch L, Nauck F, Ostgathe C, Elsner F, Bausewein C, Fuchs M, Lindena G, Neuwöhner K, Schulenberg D (2003): What are the problems in palliative care? Results from a representative survey. Support Care Cancer 11, 442-451

Rietjens J, Delden J van, Onwuteaka-Philipsen B, Buiting H, Maas P van der, Heide A van der (2008): Continuous deep sedation for patients nearing death in the Netherlands: descriptive study. BMJ $\underline{336}, 810-813$

Rothärmel S (2004): Terminale Sedierung aus juristischer Sicht: Gebotener palliativmedizinischer Standard oder heimliche aktive Sterbehilfe? Ethik Med 16, 349-357

Schildmann E, Schildmann J (2014): Palliative sedation therapy: A systematic literature review and critical appraisal of available guidance on indication and decision making. J Palliat Med 17, 601-611

Simon A (2004): Ethische Aspekte der künstlichen Ernährung bei nichteinwilligungsfähigen Patienten. Ethik Med 16, 217-228

Simon A: Ethische Probleme am Lebensende. In: Schulz S, Steigleder K, Fangerau H, Paul NW (Hrsg.): Geschichte, Theorie und Ethik der Medizin. Suhrkamp Verlag, Frankfurt am Main 2006, 446-478

Simon A (2008): Kommentar II. Ethik Med 20, 138-139

Simon A: Patientenautonomie und informed consent. In: Marckmann G (Hrsg.): Praxisbuch Ethik in der Medizin. Springer, Berlin 2015, 35-41

Simon A: Entscheidungen am Lebensende. In: Erbguth F, Jox RJ (Hrsg.): Angewandte Ethik in der Neuromedizin. Springer, Berlin 2017, 221-230

Simon A, Kar M, Hinz J, Beck D (2007): Attitudes towards terminal sedation: an empirical survey among experts in the field of medical ethics. BMC Palliat Care $\underline{6,4}$ 
Sitte T, Gronwald B, Gottschling S (2016): Palliative Versorgung statt Beihilfe zum Suizid und Tötung auf Verlangen? Über die fragliche Notwendigkeit lebensverkürzender Maßnahmen. Vollerhebung im Sinne empirischer Sozialforschung bei Palliativmedizinern in SAPV-Teams im Saarland und Hessen sowie Kinder-SAPV-Teams in Deutschland. Schmerzmedizin $\underline{32}, 25-33$

Tiirola A, Korhonen T, Surakka T, Lehto JT (2017): End-of-life care of patients with amyotrophic lateral sclerosis and other nonmalignant diseases. Am J Hosp Palliat Med $\underline{34}, 154-159$

van Oorschot B, Lipp V, Tietze A, Nickel N, Simon A (2005): Einstellungen zur Sterbehilfe und zu Patientenverfügungen: Ergebnisse einer Befragung von 727 Ärzten. DMW - Dtsch Med Wochenschr 130, 261-265

Weber M, Stiehl M, Reiter J, Rittner C (2001): Ethische Entscheidungen am Ende des Lebens. Sorgsames Abwägen der jeweiligen Situation. Ergebnisse einer Ärztebefragung in Rheinland-Pfalz. Dtsch Ärztebl 98, 3184-3188

Weber M, Strohscheer I, Samonigg H, Huber C (2005): Palliative Sedierung-eine Alternative zur Euthanasie bei unerträglichem Leid am Ende des Lebens? Med Klin 100, 292-298 


\section{Danksagung}

Mein besonderer Dank gilt meinem Doktorvater Herrn Prof. Dr. phil. A. Simon für die Überlassung des spannenden Themas und für die freundliche und geduldige Betreuung bei der Erstellung dieser Arbeit.

Weiterhin danke ich Herrn Prof. Dr. med. B. Alt-Epping für die Unterstützung bei der Erstellung des Fragebogens und für die konstruktive Beratung.

Ich möchte mich zudem bei Herrn Dr. rer. nat. A. Leha und Herrn Dr. rer. nat. F. Kück aus dem Institut für Medizinische Statistik der Universitätsmedizin Göttingen bedanken, die mich bei der statistischen Auswertung der Ergebnisse unterstützt haben.

Ich bedanke mich auch bei Herrn PD Dr. med. J.-C. Koch und bei Herrn Dr. med. B. Ilse für Anmerkungen und Kritik bei der Erstellung des Fragebogens.

Mein Dank gilt außerdem Frau Raphael aus der Informations- und Dokumentationsstelle Ethik in der Medizin für ihre Unterstützung bei der Literaturrecherche. 